\title{
Dynamics of the Antarctic Circumpolar Current: Evidence for Topographic Effects from Altimeter Data and Numerical Model Output
}

\author{
by \\ Sarah Tragler Gille \\ B.S. Yale University, \\ (1988) \\ Submitted in partial fulfillment of the \\ requirements for the degree of \\ DOCTOR OF PHILOSOPHY \\ at the
MASSACHUSETTS INSTITUTE OF TECHNOLOGY
and the \\ WOODS HOLE OCEANOGRAPHIC INSTITUTION \\ February 1995 \\ (C) Sarah Tragler Gille 1995 \\ The author hereby grants to MIT and to WHOI permission to reproduce \\ and to distribute copies of this thesis document in whole or in part.
}

Signature of Author

Joint Program in Physical Oceanography Massachusetts Institute of Technology

Woods Hole Oceanographic Institution

February, 8, 1995

Certified by

Kathryn A. Kelly Associateg Scientist Thesis Shpervisor

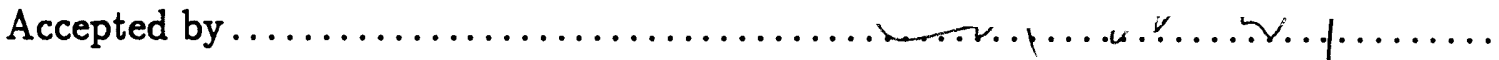
Cafl Wunsch

Chairman, Joint Committee for Physical Oceanography Massachusetts Institute of Technology Woods Hole Oceanographic Institution

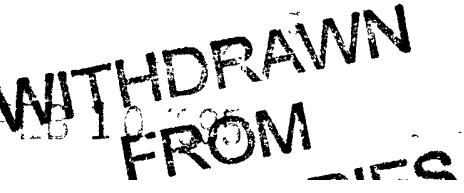




\title{
Dynamics of the Antarctic Circumpolar Current: Evidence for Topographic Effects from Altimeter Data and Numerical Model Output by \\ Sarah Tragler Gille \\ Submitted to the Massachusetts Institute of Technology/Woods Hole Oceanographic Institution Joint Program in Oceanography/Applied Ocean Science and Engineering in partial fulfillment of the requirements for the degree of Doctor of Philosophy in Oceanography
}

\begin{abstract}
Geosat altimeter data and numerical model output are used to examine the circulation and dynamics of the Antarctic Circumpolar Current (ACC).

The mean sea surface height across the ACC has been reconstructed from height variability measured by the Geosat altimeter, without assuming prior knowledge of the geoid. For this study, an automated technique has been developed to estimate mean sea surface height for each satellite ground track using a meandering Gaussian jet model, and errors have been estimated using Monte Carlo simulation. The results are objectively mapped to produce a picture of the mean Subantarctic and Polar Fronts, which together comprise the major components of the ACC. The locations of the fronts are consistent with in situ observations and indicate that the fronts are substantially steered by bathymetry. The jets have an average Gaussian width of about $44 \mathrm{~km}$ in the meridional direction and meander about $75 \mathrm{~km}$ to either side of their mean locations. The width of the fronts is proportional to $1 / f$, indicating that with constant stratification, the width is proportional to the baroclinic Rossby radius. The average height difference across the Subantarctic Front (SAF) is $0.7 \mathrm{~m}$ and across the Polar Front (PF) $0.6 \mathrm{~m}$. The mean widths of the fronts are correlated with the size of the baroclinic Rossby radius. The meandering jet model explains between $40 \%$ and $70 \%$ of the height variance along the jet axes. Bathymetric constrictions are associated with increased eddy variability, a smaller percentage of which may be explained by the meandering of the ACC fronts, indicating that propagating eddies and rings may be spawned at topographic features.

Detailed examination of spatial and temporal variability in the altimeter data indicates a spatial decorrelation scale of $85 \mathrm{~km}$ and a temporal e-folding scale of 34 days. The sea surface height variability is objectively mapped using these scales to define autocovariance functions. The resulting maps indicate substantial evidence of mesoscale eddy activity. Over 17-day time intervals, meanders of the PF and SAF appear to elongate, break off as rings, and propagate. Statistical analysis of ACC variability from altimeter data is conducted using empirical orthogonal functions (EOFs). The first mode EOF describes $16 \%$ of the variance in total sea surface height across the ACC; reducing the domain into basin scales does not significantly increase the variance represented by the first EOF, suggesting that the scales of
\end{abstract}


motion are relatively short, and may be determined by local instability mechanisms rather than larger basin scale processes. Likewise, frequency domain EOFs indicate no statistically significant traveling wave modes.

The momentum balance of the ACC has been investigated using both output from a high resolution primitive equation model and sea surface height measurements from the Geosat altimeter. In the Semtner-Chervin general circulation model, run with approximately quarter-degree resolution and time varying ECMWF winds, topographic form stress is the dominant process balancing the surface wind forcing. Detailed examination of form stress in the model indicates that it is due to three large topographic obstructions located at Kerguelen Island, Campbell Plateau, and Drake Passage. In order to reduce the effects of standing eddies, the model momentum balance is considered in stream coordinates; vertically integrated through the entire water column, topographic form drag is the dominant balance for wind stress. However, at mid-depth the cross-stream momentum transfer is dominated by horizontal biharmonic friction. In the upper ocean, horizontal friction, mean momentum flux divergence, transient momentum flux divergence, and mean vertical flux divergence all contribute significantly to the momentum balance. Although the relative importance of individual terms in the momentum balance does not vary substantially along streamlines, elevated levels of eddy kinetic energy are associated with the three major topographic features. In contrast, altimeter data show elevated energy levels at many more topographic features of intermediate scales, suggesting that smaller topographic effects are better able to communicate with the surface in the real ocean than in the model. Transient Reynolds stress terms play a small role in the the overall momentum balance; nonetheless, altimeter and model measurements closely agree, and suggest that transient eddies tend to accelerate the mean flow, except in the region between the major fronts which comprise the ACC.

Potential vorticity is considered in the model output along Montgomery streamfunction. Even at about $1000 \mathrm{~m}$ depth, it varies in response to wind forcing, largely as a result of changes in vertical stratification, indicating that forcing and dissipation do not locally balance in the Southern Ocean. In order to compare model and altimeter potential vorticity estimates, two different proxies for potential vorticity on surface streamlines are considered. Both proxies show very similar results for model and altimeter, suggesting that differences in surface streamlines estimated by the altimeter and the model are not significant in explaining the Southern Ocean flow. The proxies are both roughly conserved along surface height contours but undergo substantial jumps near topographic features. However, they cannot capture stratification changes which may be critically important to the overall potential vorticity balance.

Thesis Supervisor: Dr. Kathryn A. Kelly

Title: Associate Scientist, Physical Oceanography, Woods Hole Oceanographic Institution 


\section{Acknowledgments}

I am grateful to my advisor Kathie Kelly, both for the initial suggestion to consider the Southern Ocean and for continued guidance and encouragement throughout the course of this research. Committee members Terry Joyce, John Marshall, Mike McCartney, and John Toole have all given valuable suggestions. Xin Huang kindly agreed to chair my defense. In addition, Bob Chervin read and commented on a draft of this thesis, and Steve Lentz provided a useful critique of an early draft of Chapter 2. Harry Bryden, Glenn Flierl, Bo Qiu, and Carl Wunsch also offered useful comments during this work.

The computing resources of the remote sensing group at WHOI have been extremely important. Mike Caruso's computer assistance has been invaluable; he's managed to keep our system running beautifully while simultaneously answering my barrage of questions. Sandy Singh has also readily advised on graphics and unix subtleties. In addition, some of the objective mapping was performed using supercomputer facilities at the National Center for Atmospheric Research (NCAR), which is operated by the University Corporation for Atmospheric Research and is sponsored by the National Science Foundation.

The numerical model output used in chapters 4 and 5 was generously provided by Bob Chervin and Tony Craig at NCAR and Bert Semtner and Robin Tokmakian of the Naval Postgraduate School. All of them have readily answered my many questions. In particular, Bob Chervin arranged for NCAR's Climate and Global Dynamics Division to sponsor a visit to Boulder, and Tony Craig spent a considerable amount of time orienting me to the intricacies of the numerical model and the software available to analyze its output.

Other data have come from a variety of sources. Geosat data were provided by the National Oceanographic Data Center. Dirk Olbers of the Alfred Wegener Institute in Bremerhaven, Germany, made his gridded data set publicly available, and Bruce Huber of Lamont-Doherty Earth Observatory provided the gridded atlas data of Gordon et al. [1982]. Numerous other investigators provided information on hydrographic data from specific cruises, most of which has been used for general comparison but is not specifically discussed in this thesis.

Funding for this research was provided by an Office of Naval Research graduate student fellowship and National Aeronautics and Space Administration contract NAGW-1666.

Countless friends-housemates, fellow Joint Program students, and othershave been essential supporters. My graduate school classmates might merit acknowledgment even if they had not coerced me into writing this sentence: together they provided a valued mixture of teasing, scientific discussion, and moral support. I'm also grateful to the many other Joint Program students, past and present, with whom I've discussed this research, in particular Antonietta Capotondi, Anand Gnanadesikan, Barry Klinger, Joe LaCasce, Young-Gyu Park, Jamie Pringle, Elise Ralph, and Lip- 
ing Wang. In addition, Stefan Llewellyn Smith has been an extraordinary friend and proofreader.

Finally I thank my parents, who might have been knowledgeable enough to have prodded me onward or to have discouraged me altogether, but who instead permitted me to make my own decisions. Their unconditional support throughout years of education has been wonderful. 


\section{Contents}

$\begin{array}{ll}\text { Abstract } & 3\end{array}$

Acknowledgments $\quad 5$

1 Introduction $\quad 11$

2 Mean sea surface height of the ACC 17

2.1 Introduction . . . . . . . . . . . . . . . . . 17

2.2 Processing the Geosat Data . . . . . . . . . . . . . . . . . 21

2.2.1 Collinear Methods and Data Corrections . . . . . . . . . 21

2.2.2 Reconstructing Mean Sea Surface Height . . . . . . . . . . 25

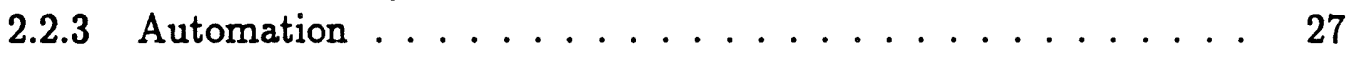

2.3 Error Estimation . . . . . . . . . . . . . . . . 30

2.3.1 Assessing Bias From "Perfect" Data . . . . . . . . . . . . 31

2.3.2 Assessing Repeatability (Precision) . . . . . . . . . . 32

2.4 Mapping Regional Results . . . . . . . . . . . . . . 38

2.4.1 Mean Sea Surface Height . . . . . . . . . . . . . 38

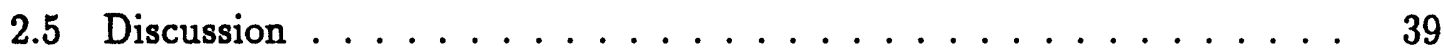

2.5.1 Jet Statistics . . . . . . . . . . . . . . . 39

2.5.2 Height Variance ................. 50

2.5.3 Mean Mapped Sea Surface Height . . . . . . . . . . . . 50

2.5.4 South of Australia, $100^{\circ}-140^{\circ} \mathrm{E} \ldots \ldots . . \ldots 53$

2.5.5 Tasman Sea-Campbell Plateau, $140^{\circ}-180^{\circ} \mathrm{E} \ldots \ldots . . . . .53$

2.5.6 Southwest Pacific, $180^{\circ}-130^{\circ} \mathrm{W} \ldots \ldots . . \ldots 54$

2.5.7 Southeast Pacific, $130^{\circ}-80^{\circ} \mathrm{W}$............. 54

2.5.8 Drake Passage, $80^{\circ}-55^{\circ} \mathrm{W}$. . . . . . . . . . . 54

2.5.9 Southwest Atlantic, $55^{\circ}-0^{\circ} \mathrm{W} \ldots \ldots . \ldots 55$

2.5.10 Southeast Atlantic and the Southwest Indian Oceans, $0^{\circ}-40^{\circ} \mathrm{E} \quad 56$

2.5.11 South Indian Ocean, $40^{\circ}-100^{\circ} \mathrm{E} \ldots . . . . . . . . .56$

2.5.12 Geographic Summary . . . . . . . . . . . . . 57

2.6 Summary ............................ 57 
2.7 Appendix: Height Variance and Mean Velocity . . . . . . . . . . 58

3 Variability $\quad 65$

3.1 Introduction . . . . . . . . . . . . . . 65

3.2 Scales of Variability . . . . . . . . . . . . . . . . 68

3.2.1 Time Varying Sea Surface Height . . . . . . . . . . 68

3.2.2 Aliasing and Altimeter Sampling . . . . . . . . . . . 74

3.2.3 Local Eddy Processes . . . . . . . . . . . . . . . . . 77

3.3 Seeking Coherence in Variability . . . . . . . . . . . . 80

3.3.1 Time Domain Variability . . . . . . . . . . . . . . . 80

3.3.2 Frequency Domain Variability . . . . . . . . . . . . . 92

3.4 Discussion: Interpreting Incoherence $\ldots \ldots \ldots \ldots \ldots \ldots$

3.5 Summary $\ldots \ldots \ldots \ldots \ldots \ldots \ldots \ldots$

3.6 Appendix: Estimating Velocities and Errors . . . . . . . . . 99

4 Momentum 101

4.1 Introduction . . . . . . . . . . . . . . . . . 101

4.2 Background: Processes Balancing Wind . . . . . . . . . . . . . . 102

4.2 .1 Form Drag . . . . . . . . . . . . . . . . . . . . . 104

4.2.2 Processes Considered in Past Studies . . . . . . . . . . 111

4.3 Momentum Information from Model Output . . . . . . . . . . . . 115

4.4 Vertically Integrated Momentum Balance . . . . . . . . . . . . . . 119

4.4 .1 A Meridional Slice . . . . . . . . . . . . . . . . . . 119

4.4 .2 Zonally Averaged Balance . . . . . . . . . . . . . . . . 119

4.4.3 Average Balance Along Streamlines. . . . . . . . . . . . . 122

4.4.4 Local Balance Along Streamlines. . . . . . . . . . . . . . 128

4.4.5 Form Drag Along Streamlines. . . . . . . . . . . . . . . 131

4.5 Mid-Depth Momentum Balance . . . . . . . . . . . . . . . . . 134

4.5 .1 Zonally Averaged Balance . . . . . . . . . . . . . . 134

4.5.2 Average Balance along Streamlines . . . . . . . . . . . 134

4.5.3 Balance along Streamlines . . . . . . . . . . . . . 140

4.6 Surface Momentum Balance . . . . . . . . . . . . . . . . . . . . . 142

4.6 .1 Zonally Averaged Balance . . . . . . . . . . . . . . . 142

4.6.2 Average Balance along Streamlines . . . . . . . . . . . . . 144

4.6.3 Balance along Streamlines . . . . . . . . . . . . . . . 148

4.7 Surface Eddy Kinetic Energy _ . . . . . . . . . . . . . . . 148

4.8 Surface Reynolds Stresses . . . . . . . . . . . . . . . . . 156

4.9 Summary . . . . . . . . . . . . . . . . . . 160

4.10 Appendix: Accounting for non-linearities in the equation of state. . . 163 
5 Potential Vorticity 165

5.1 Introduction . . . . . . . . . . . . . . . . 165

5.2 Potential Vorticity on Isopycnals . . . . . . . . . . . . . 168

5.2.1 Defining Potential Vorticity . . . . . . . . . . . . 168

5.2.2 Forcing Terms on Streamlines . . . . . . . . . . . . . . . 174

5.2.3 Potential Vorticity on Montgomery Streamfunction . . . . . 175

5.2.4 Isopycnal Potential Vorticity Estimates from Data . . . . . . . 180

5.3 Potential Vorticity on Surface Streamlines . . . . . . . . . . . 185

5.3.1 An Equivalent Barotropic Form . . . . . . . . . . . . 185

5.3.2 Model and Data Surface Potential Vorticity . . . . . . . . 190

5.4 Summary . . . . . . . . . . . . . . . . . . . 198

5.5 Appendix: Alternative Potential Vorticity Formulation . . . . . . . . 199

6 Summary Discussion $\quad 203$

$\begin{array}{ll}\text { Bibliography } & 208\end{array}$ 


\section{Chapter 1}

\section{Introduction}

Oceans play a vital role in regulating climate on Earth. Covering over half the surface of the planet with a total mass 270 times greater than that of the atmosphere, the oceans serve as great reservoirs of heat and water [Gill, 1982]. Fundamental to understanding how the oceans influence climate is identifying how heat and freshwater are transported through the world's oceans. The Antarctic Circumpolar Current (ACC), which links the Atlantic, Indian, and Pacific Oceans, carries roughly $130 \times 10^{6}$ $\mathrm{m}^{3} \mathrm{~s}^{-1}$ of water, and has the potential to play a significant part in the ocean climate system. Only within the Southern Ocean, at latitudes poleward of about $35^{\circ} \mathrm{S}$, are all three oceans able to communicate. The role of the ACC is closely tied to its dynamics: if the ACC interacted little with its surroundings, it would act as a barrier to heat transport by impeding exchange with the Antarctic shelf while having no real impact on the heat budgets of the three major ocean basins. Conversely, the extent to which the ACC exchanges mass, momentum, heat and other properties with the three major oceans and the Antarctic shelf region is a measure of its actual role in climate.

Ongoing investigations of North Atlantic Deep Water renewal (dubbed the "conveyer belt" by Broecker $[1987,1991])$ have enunciated one clear example of the ACC's function in inter-basin exchange processes. Gordon [1986] outlined a mech- 
anism by which NADW is formed through deep convection of warm salty surface processes in the northern Atlantic and spreads southward within a deep western boundary current. Eventually the water enters the deep ACC to mix with Antarctic deep waters and spread throughout the global ocean, as suggested by the isopycnal analysis of Reid and Lynn [1971]. In his scheme, upwelling occurs in the tropical Pacific and Indian Oceans, where water acquires elevated salinity. This upper level water flows into the Atlantic to replace the outflowing NADW. Gordon [1986] initially emphasized the importance of the "warm water" route for this upper level return flow through the Indonesian Archipelago and around the southern tip of Africa via the Agulhas Retroflection.

Recent data analyses have examined the flow patterns suggested by Gordon. In an inverse model of the South Atlantic, Rintoul [1991] was not able to justify a significant net transport of Indian Ocean thermocline water into the South Atlantic; his model instead indicated that intermediate water from Drake Passage replaces NADW exiting into the Indian Ocean. Similarly, in an inversion of Southern Hemisphere zonal hydrographic sections, Macdonald [1993] found that South Atlantic heat budgets were more consistent with the Drake Passage route and that the evidence did not support substantial flow through the Indonesian Passage. Numerical model results are consistent with these data analyses in suggesting that most of the upwelling necessary for NADW formation occurs in the circumpolar region [Toggweiler and Samuels, 1994]. Schmitz [1994], in a detailed review, suggested a process by which water upwells and mixes gradually along an extended trajectory through the Southern Ocean. Roughly two-thirds of the upper layer flow into the Atlantic arrives through Drake Passage as colder intermediate water and, as it flows northward within the Atlantic, gradually warms and upwells to the thermocline.

In providing the intermediate water from all three ocean basins necessary for NADW formation, the ACC is directly coupled to the global oceanic heat budget. Georgi and Toole's [1982] analysis of temperature transport through hydrographic 
sections spanning the three major choke points of the Southern Ocean indicated that the ACC loses heat as it travels through the Atlantic and Pacific Oceans and gains heat within the Indian Ocean. While one might be able to explain the observed ACC temperature changes entirely with air-sea heat exchanges, generally accepted hypotheses assume substantial heat loss to the atmosphere throughout the Southern Ocean. Thus, variations in heat content of the ACC rely on significant and inhomogeneous poleward heat transport, due to eddy processes or localized current inputs. If we are to understand how the ACC couples into the global ocean climate system, then we must investigate the dynamics which govern its heat and momentum budgets and the temporal fluctuations which account for its variations.

Evaluations of Southern Ocean dynamics have suggested that the meridional heat transport is directly linked to the zonal momentum balance [Johnson and Bryden, 1989; Marshall et al., 1993]. With no meridional boundaries to support net zonal pressure gradients or intense western boundary currents, the circumpolar system cannot rely on the same mechanisms as more northerly basins to counterbalance the enormous surface wind forcing which drives it. This has meant that conceptualizations of Southern Ocean dynamics are distinctly different from those for regionally bounded basins like the North Atlantic and, therefore, are correspondingly less well understood. Because of its circumpolar extent, the ACC bears certain resemblances to the atmospheric jet stream; however, since the ACC is subject to surface wind forcing as well as bottom topographic stresses, its momentum balance differs somewhat from that of the jet stream. Form drag, resulting from partial bathymetric obstruction, is the primary process invoked to explain the ACC response to wind. Johnson and Bryden [1989] suggested that interfacial form drag due to transient displacement of isopycnal surfaces is responsible for transferring momentum vertically from the surface to deep levels where topographic form drag takes effect. The mechanism they proposed therefore directly tied the strength of the ACC to the magnitude of the meridional heat transfer due to transient eddies. Marshall et al. [1993] considered 
this mechanism, assuming constant stratification and constant depth, to estimate a poleward heat flux of $0.24 \times 10^{15} \mathrm{~W}$, which does not differ substantially from deSzoeke and Levine's [1981] estimate of $0.3 \times 10^{15} \mathrm{~W}$, determined from hydrographic observations. This suggested not only that the numerous mesoscale eddies in the Southern Ocean are an essential part of the system's dynamics, but also that understanding the ACC dynamics may ultimately help explain the global heat budget.

Because of the Southern Ocean's role in inter-ocean communication, fluctuations in its characteristics may ultimately affect the climatology of all the world's oceans; yet despite their importance in climate and general circulation, the dynamics of the Southern Ocean remain somewhat puzzling. Remote location and vast size have made the Southern Ocean difficult to sample observationally. The most detailed hydrographic survey was the series of Eltanin cruises of the early 1970s, which resulted in meridional sections around three-quarters of the Southern Ocean. Nonetheless, 65 years of observations have produced only a sparse grid of measurements. The total number of hydrographic stations assembled to produce the most recent atlas [Olbers et al., 1992] totaled less than 40,000 for a region covering about one third of the global ocean surface area. Moreover, the measurements are concentrated in the region around Drake Passage $\left(60^{\circ} \mathrm{W}\right)$ while other areas such as the eastern Pacific Ocean $\left(130^{\circ} \mathrm{W}\right.$ to $\left.90^{\circ} \mathrm{W}\right)$ are sparsely sampled, as indicated in Figure 1.1.

Elusive as the ACC may be, the goal of this study is to pinpoint the critical processes governing it, with particular emphasis on the role of topography. The chapters which follow address questions of ACC circulation using a combination of Geosat altimeter data, hydrographic data from the recently compiled atlas by Olbers et al. [1992], and output from a high resolution primitive equation model [Semtner and Chervin, 1992]. Chapter 2 provides a detailed introduction to the observational characteristics of the ACC. The multiple jet structure seen in hydrographic and current meter observations is then employed to reconstruct mean sea surface height from variability measured by the Geosat altimeter. The results, which are consistent with 


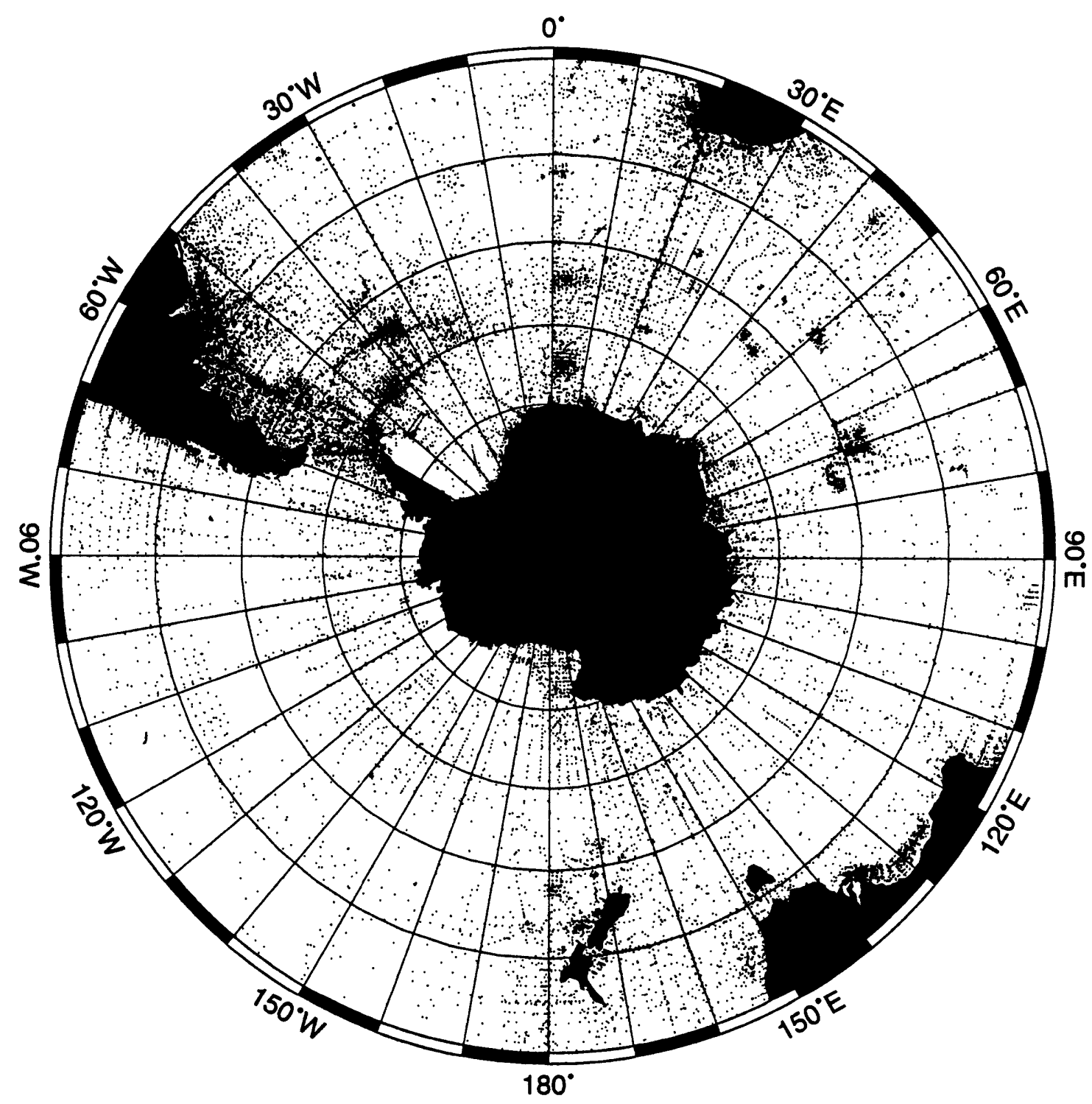

Figure 1.1: Locations of all hydrographic stations assembled to develop the atlas by Olbers et al [1992]. A total of 38,816 stations poleward of $30^{\circ} \mathrm{S}$ were used. They were primarily collected during the time period from 1924 to 1991. 
the presence of continuous narrow jets, indicate that the path of the ACC jets are closely steered by bathymetric features. Chapter 3 examines ACC variability measured by the altimeter. Chapter 4 uses Semtner-Chervin model output to examine the momentum balance of the ACC in both zonally averaged and stream coordinates, and interprets these results in the context of altimeter measurements. In Chapter 5, potential vorticity along streamlines is examined in model output and altimeter measurements. Finally the overall results are summarized in Chapter 6 . These chapters employ a range of methods to probe the fundamental dynamics governing the Antarctic Circumpolar Current. Each of them shows evidence that the topographic ridges in the Southern Ocean play a substantial role in controlling transport, variability, and the mean path of the flow. 


\section{Chapter 2}

\section{Mean sea surface height of the}

\section{$\mathrm{ACC}$}

\subsection{Introduction}

The Southern Ocean, the part of the world's oceans south of $35^{\circ} \mathrm{S}$, makes up over a quarter of the global ocean surface area. ${ }^{1}$ It carries a strong circumpolar flow, the Antarctic Circumpolar Current (ACC), with a total transport of about $130 \times 10^{6} \mathrm{~m}^{3}$ $\mathrm{s}^{-1}$, which represents most of the mass transport between the Atlantic, Pacific, and Indian Oceans.

Despite its substantial transport, the Southern Ocean remains one of the more enigmatic of ocean systems. Owing to remote location, vast size, and consistently poor weather, it has not been sampled extensively using traditional methods. Southern Ocean hydrographic station separations along synoptic sections are often $100 \mathrm{~km}$ or more, and sections may be separated spatially by several hundred kilometers and temporally by many years, meaning that mesoscale features are poorly resolved. His-

\footnotetext{
${ }^{1}$ This chapter duplicates "Mean sea surface height of the Antarctic Circumpolar Current from Geosat data: Method and application" by S. T. Gille, J. Geophys. Res., 99 18,255-18,273, 1994. Copyright by the American Geosphysical Union. Reprinted with permission. All additions to the published text have been included as footnotes.
} 
toric hydrographic data for the region have been assembled by Gordon et al. [1982] and more recently by Olbers et al. [1992] to produce relatively consistent, though heavily smoothed and nonsynoptic, pictures of the ACC frontal structure. On the basis of available observations, the ACC is often conceptualized as containing a series of sharp temperature and density fronts, each separating two distinct water masses and supporting a jetlike geostrophic velocity structure [Nowlin and Klinck, 1986]. Although some investigators have considered the fronts primarily as water mass separations and have concentrated on temperature and salinity characteristics of the fronts, for the purposes of this investigation they will be identified based on their intensified geostrophic velocities. From north to south, these fronts are referred to as the Subtropical Front (STF), the Subantarctic Front (SAF), the Polar Front (PF), and the Continental Water Boundary (CWB) [Nowlin and Klinck, 1986]. The northernmost front, the STF, is blocked by continental boundaries and so carries no circumpolar flow, while the CWB accounts for only about $15 \%$ of the ACC transport. (Recent work by Orsi [1993] has also suggested the presence of a southern ACC front located between the PF and the CWB.) Together the central SAF and PF carry the bulk of the transport relative to $2500 \mathrm{~m}$ through the Drake Passage [Nowlin and Clifford, 1982]. Their mean locations, indicated in Figure 2.1, were estimated by identifying dynamic height gradient maxima in Gordon et al.'s [1982] atlas data and smoothed using a biharmonic spline.

Although the observations suggest that the fronts have meandering mesoscale structures, outside of the Drake Passage few moorings have been deployed and few sections have been repeated on a regular basis, so that the data archives contain little information about means and variances of jet locations and transports. As part of International Southern Ocean Studies (ISOS), current meters [Whitworth et al., 1982], heavily instrumented transport moorings [Whitworth, 1983; Whitworth and Peterson, 1985] and pressure gauges [Wearn and Baker, 1980] were deployed across the Drake Passage to monitor the variability of the ACC. Results indicated that 


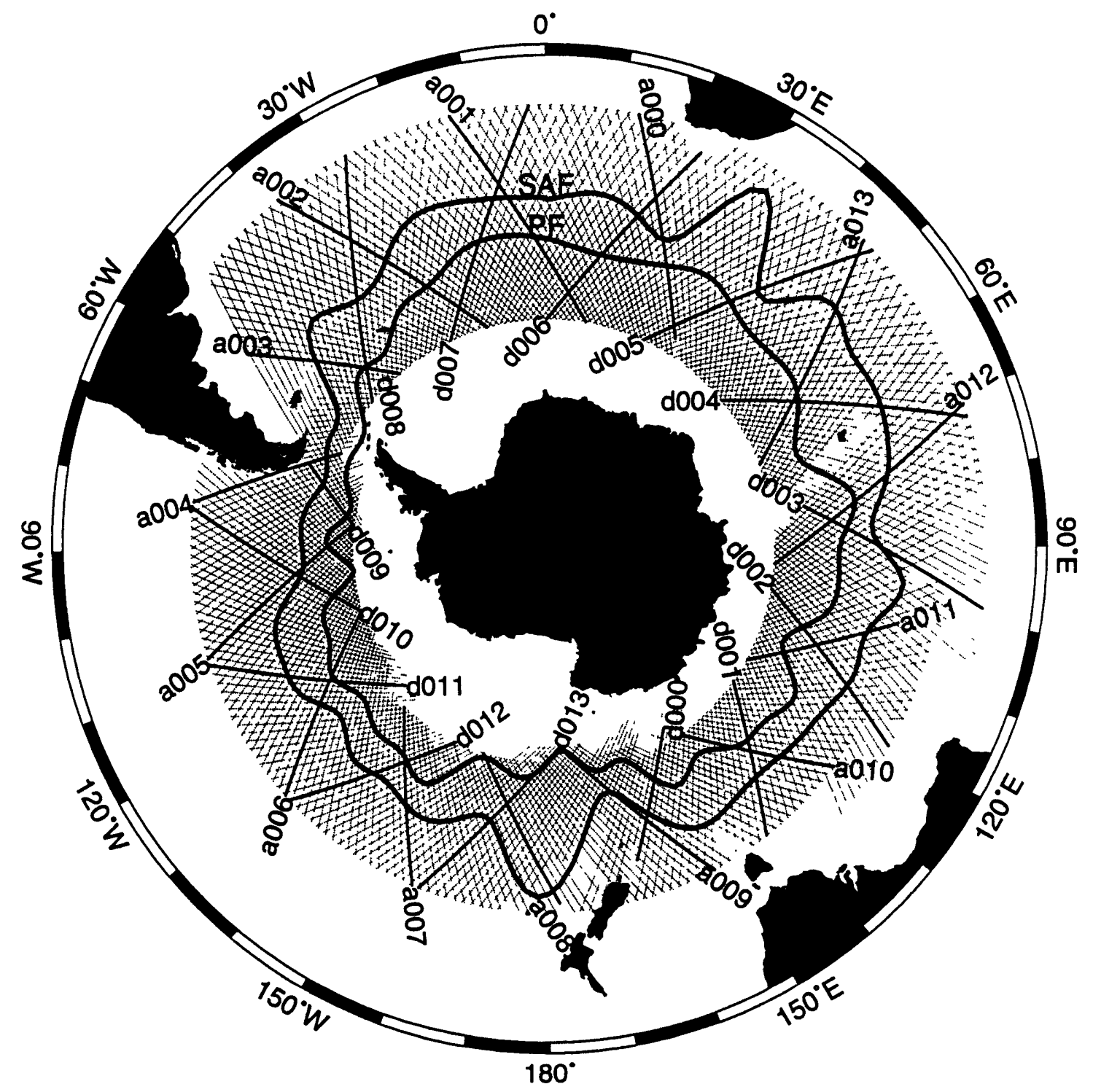

Figure 2.1: Mean locations of the Subantarctic Front (SAF) and Polar Front (PF), estimated from Gordon et al.'s [1982] atlas data, are depicted as bold lines. The medium lines indicate the locations of the 28 track segments, labeled a000 through a013 and d000 through d013, used to select appropriate corrections to apply and for the Monte Carlo error estimations. The lightest lines indicate coverage provided by the 244 ascending and 244 descending Geosat track segments used to reconstruct the mean sea surface height for the Southern Ocean. The outer edge of the map is at $30^{\circ} \mathrm{S}$. 
the baroclinic transport is relatively stable, while the barotropic transport fluctuates on the order of $50 \times 10^{6} \mathrm{~m}^{3} \mathrm{~s}^{-1}$, with a standard deviation of $10 \times 10^{6} \mathrm{~m}^{3} \mathrm{~s}^{-1}$. In a few cases, wave growth and ring formation events have been observed in roughly synoptic expendable bathythermography (XBT) and hydrographic surveys at Drake Passage [Joyce and Patterson, 1977; Peterson et al., 1982] and south of Australia [Savchenko et al., 1978], but little information has been available to investigate variability throughout the Southern Ocean. One tool which has proved useful for synoptic studies of mesoscale structures in the Gulf Stream and the Kuroshio, advanced very high resolution radiometer (AVHRR) sea surface temperature data, is of extremely limited use in the Southern Ocean because of the nearly constant cloud cover in the region.

Altimetry provides global measurements of sea surface height (SSH) with relatively dense spatial and temporal coverage. The Geosat Exact Repeat Mission (November 1986 through September 1989) passed over the same collinear tracks every 17 days, providing measurements with 7 - $\mathrm{km}$ along-track resolution and about 100 $\mathrm{km}$ spacing between tracks. The dominant signal in the altimeter data, the Earth's gravitational field (referred to as the geoid), is not known with sufficient accuracy to permit its simple removal from the altimeter measurements. Most investigators have chosen to remove the mean from the data, in essence subtracting the mean SSH along with the geoid, to leave only the variability. Studies by Chelton et al. [1990], Morrow et al. [1992], and Johnson et al. [1992] have taken advantage of Geosat altimeter measurements to investigate SSH variability in the Southern Ocean. This chapter will take a slightly different strategy by using the variability along with a Gaussian model of the zonal jet structure to reconstruct the mesoscale mean SSH field. Because of the large quantity of data provided by the altimeter and the sampling problems inherent in the altimeter orbit patterns, a robust and automated data processing scheme has been developed. This approach will allow us to address several lingering issues in the description of basic ACC circulation: Are the altimeter measurements consistent with 
the idea that the ACC contains continuous narrow jets? Where are these jets located, how far do they meander, how wide are they, and what is the height difference across the jets?

This chapter is organized as follows: section 2.2 (along with the appendix) outlines the basic methodology used to estimate the mean SSH from Geosat data. Section 2.3 describes the Monte Carlo simulation approach to error analysis. Section 2.4 presents the objectively mapped SSH obtained using this approach. Sec-

tion 2.5 focuses specifically on the results, discussing the statistics of jet meandering and carefully examining the reconstructed mean height field. The results are summarized in section 2.6 .

\subsection{Processing the Geosat Data}

\subsubsection{Collinear Methods and Data Corrections}

Altimeter SSH measurements consist of three main components: the geoid, which may change by $30 \mathrm{~m}$ across the width of the Southern Ocean; the time-invariant mean oceanographic SSH of 1 to $1.5 \mathrm{~m}$ amplitude; and the time-varying fluctuations, typically 0.3 to $0.6 \mathrm{~m}$ for mesoscale phenomena. Additional signals are due to orbit error, atmospheric effects, tides, and instrument noise. The basic collinear data processing method may be summarized as follows: For each satellite pass along a given ground track, $30^{\circ}$ arcs are selected and missing and out-of-range data points are removed. Data from regions less than $2000 \mathrm{~m}$ deep are eliminated because the tidal model is not reliable in shallow water. (This step is omitted in a few cases, notably for ascending tracks near Campbell Plateau, where near-coastal data are needed to identify the ACC.) Selected corrections are applied to the raw data, obvious anomalous spikes are removed, and the data are splined to a common grid. Orbit errors are removed by fitting the height profile to a sinusoid with a wavelength corresponding to one satellite revolution about the Earth. This fit also removes long-wavelength 
tidal errors and other measurement errors. To prevent regions of large mesoscale variability from biasing the orbit correction, the least squares fit is weighted by the inverse of the height variance. Next the mean height for all of the satellite passes over each point along the orbit is computed and subtracted from the measured height for each satellite pass to produce a time series of residual SSH. The resulting along track data are low-pass filtered. Further details about the collinear method are provided by Caruso et al. [1990]. A segment of a filtered time series for one collinear track is shown in Figure 2.2 (left).

Standard data corrections are available for solid earth tides, ocean tides, ionospheric effects, dry tropospheric travel time delays (from both the European Centre for Medium-Range Weather Forecasts' (ECMWF) operational forecast model and the Fleet Numerical Oceanographic Center's (FNOC) data archives), and wet tropospheric travel time delays (from the special sensor microwave imager (SSMI) and FNOC) [Cheney et al., 1991]. To test their usefulness, each of the corrections was applied to data from the 28 ground tracks identified by dark lines in Figure 2.1. The mean change in the height variance, averaged for all points along the 28 tracks, was used to gauge the effectiveness of each correction, as suggested by Campbell [1988]. Corrections were applied if they reduced the variance in the residual height by more than the point-to-point along-track variance, $9 \times 10^{-4} \mathrm{~m}^{2}$, which is an estimate of the instrument precision [Chelton et al., 1990]. The results, summarized in Table 2.1, indicate that only the ocean tide, dry troposphere, and inverted barometer corrections met this criterion. By a small margin, the ECMWF dry troposphere correction was more effective than the FNOC correction. The electromagnetic (EM) bias correction significantly increases the variance and is apparently ineffective. In comparison, for the less variable northeast Pacific, Campbell [1988] applied just one correction, concluding that only the ocean tide consistently reduced the data variance. 


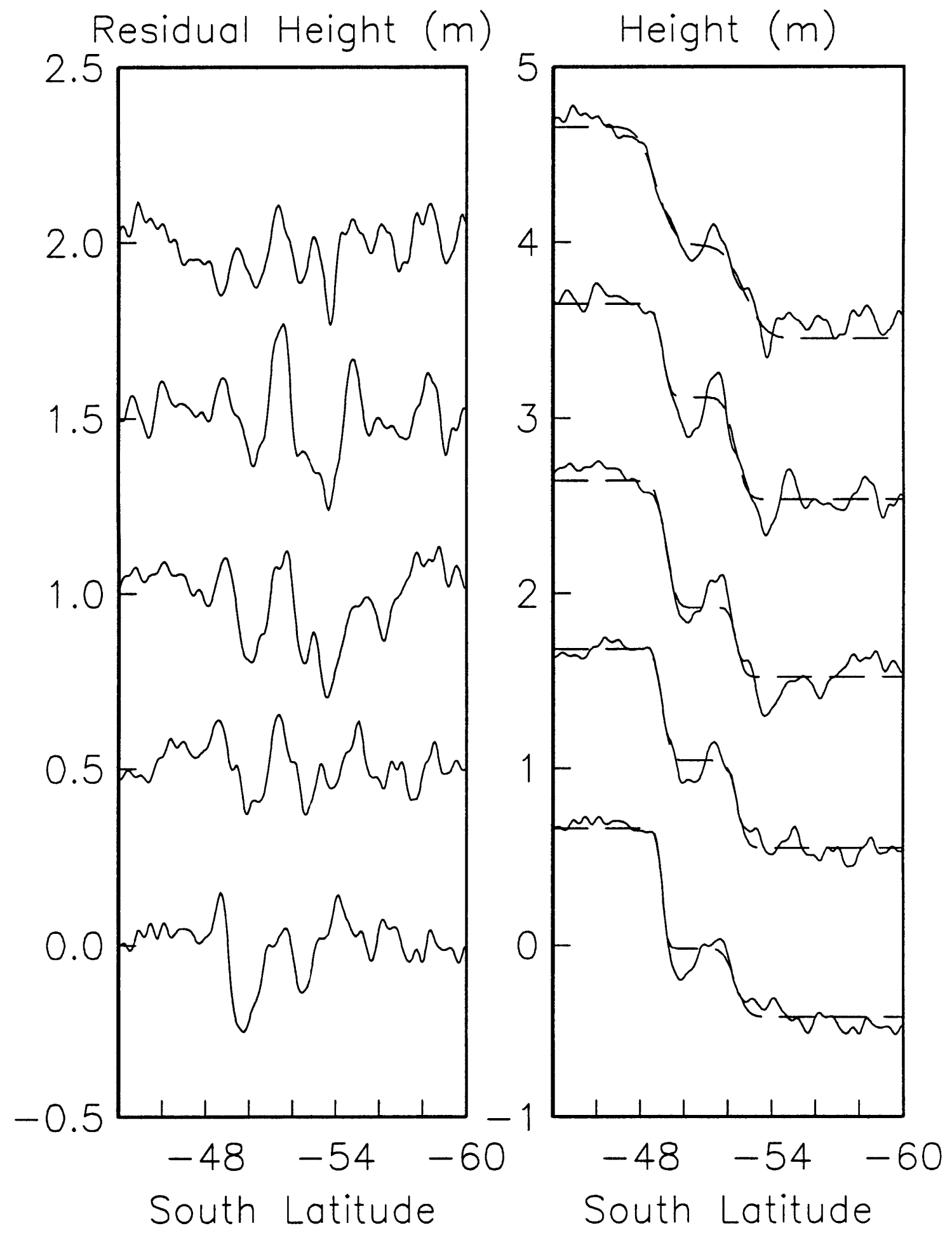

Figure 2.2: An example time series of (left) residual height data (in meters), and (right) residual height data added to the reconstructed mean (in meters), for the first five satellite passes along track d001. The dashed lines in the right panel indicate instantaneous modeled height from the error function model. 


\begin{tabular}{|l|r|}
\hline Correction & $\begin{array}{r}\text { Mean } \\
\text { Variance } \\
\text { Change } \\
10^{-4} \mathrm{~m}^{2}\end{array}$ \\
\hline None & 0.00 \\
Solid earth tide & -3.70 \\
Ionospheric & 1.26 \\
Wet troposphere (FNOC) & -3.97 \\
Wet troposphere (SSMI) & -3.73 \\
EM bias & 94.41 \\
Inverted barometer & -156.63 \\
Dry troposphere (FNOC) & -50.78 \\
Dry troposphere (ECMWF) & -54.95 \\
Ocean tides & -172.55 \\
Applied corrections & -287.22 \\
All available & -292.43 \\
\hline
\end{tabular}

Table 2.1: The Effect of Each of the Suggested Corrections on the Variance of 28 Ground Tracks. Ground tracks are referred to as d000 through d013 and a000 through a013 in Figure 2.1. Numerical values represent the mean change in the variance for all of the points in the 28 tracks. 


\subsubsection{Reconstructing Mean Sea Surface Height}

Although the mean is removed from the measured SSH, it is nonetheless a dynamically important quantity. Kelly and Gille [1990] outlined a technique which permits reconstruction of the mean SSH from the variability of a meandering jet. The technique, which has subsequently been refined and applied by Kelly [1991], Qiu et al. [1991], and Qiu [1992], uses the basic assumption that a velocity jet has a Gaussian structure. In the case of the ACC, measurements in the Drake Passage suggest that the SAF and PF may be treated as separate Gaussian jets [e.g. Peterson et al., 1982], as shown in Figure 2.3.

(The CWB and any additional ACC fronts are estimated to be too far south to be consistently identified by the altimeter.) Using the geostrophic relation, the velocity is integrated to produce a SSH section which is an error function for each front:

$$
\begin{gathered}
h(x, t)=\int_{a_{2}}^{x} \frac{a_{1}}{\sqrt{2 \pi} a_{3}} \exp \left(-\frac{\left(x^{\prime}-a_{2}\right)^{2}}{2 a_{3}^{2}}\right) d x^{\prime} \\
=\frac{a_{1}}{2} \operatorname{erf}\left(\frac{x-a_{2}}{\sqrt{2} a_{3}}\right)
\end{gathered}
$$

centered at $a_{2}$, with width $a_{3}$, and total height change across the jet $a_{1}$.

Strong residual velocity features (or bumps in the residual height) are used to estimate error function parameters for the height $a_{1}$, location $a_{2}$, and width $a_{3}$, of the jet at the time of each satellite pass. Modeled heights for each satellite repeat are averaged to estimate mean height along the satellite ground track, which is added to the residual height data. Using a nonlinear fitting scheme (outlined below), the parameters of the error function are iteratively improved to provide better estimates of the true SSH. By repeating the sequence (constructing height profiles from parameters, averaging them to estimate the mean, adding the mean to residual data, and refitting the parameters), the mean height field estimate is fine tuned to best represent the data. In the right panel of Figure 2.2, the reconstructed mean plus residual 

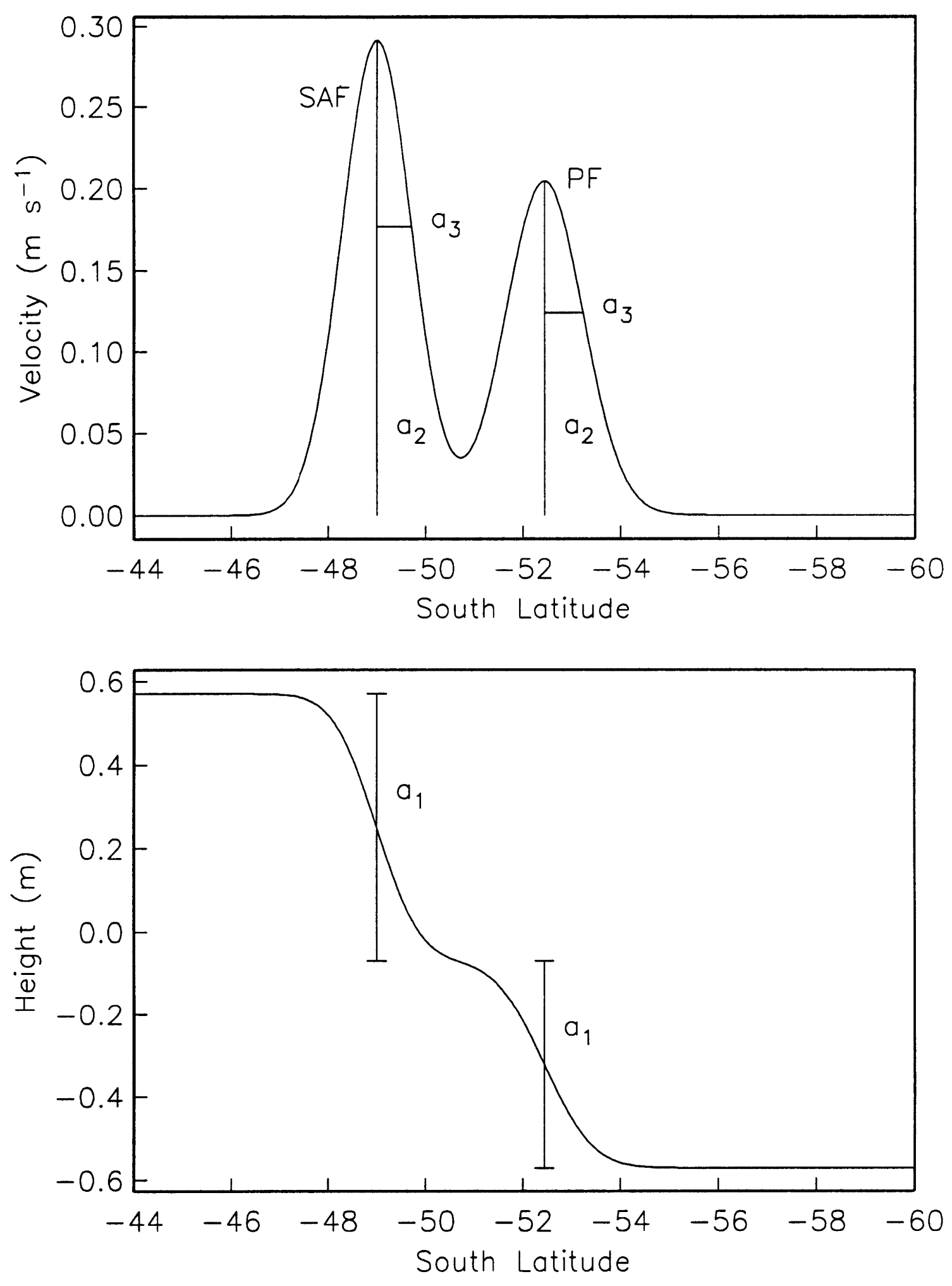

Figure 2.3: Schematic showing parameters defining sea surface height (SSH) error function. 
height (solid line) contains substantial eddy variability, while the instantaneous fit (dashed line) smoothly captures the principal jet structure of the ACC.

The success of this approach is contingent on several key criteria. First, the residual height bumps corresponding to the jet must be large enough to be detected by the altimeter. Second, the jet studied should have a roughly Gaussian velocity structure. Third, it must meander substantially; Qiu [1992] found that the standard deviation of the position parameter, $\sigma_{a_{2}}$, should be at least twice the mean width parameter, $\overline{a_{3}}$, if a reliable estimate of the mean height field is to be obtained. Simulations conducted for the present study indicate that this criterion may be relaxed if the amplitude and width fluctuate along with the jet position. In the Kuroshio and in the Gulf Stream, these criteria are normally fulfilled. The strong single jets corresponding to $1-\mathrm{m}$ height jumps are easily identified, and plausible height fields are produced, as confirmed by acoustic Doppler current profiler data [Kelly et al., 1991] and by inverted echo sounder thermocline depth fluctuations [Kelly and Watts, 1994].

The ACC is potentially more difficult to study than the northern hemisphere western boundary currents because of its multiple jet structure and smaller associated sea surface topography fluctuations. The height jump across each of the ACC fronts is about $0.5 \mathrm{~m}$ [Gordon et al., 1978] (henceforth GMB78), compared with $1 \mathrm{~m}$ for the Gulf Stream. Even so, the criteria required for a reliable reconstruction of the height field are nearly always satisfied. Residual height jumps along the ACC axes are large enough to be detected by the altimeter. The error analysis in section 2.3 indicates that the mean height is successfully reconstructed to within about a $10 \%$ error, without significant systematic bias.

\subsubsection{Automation}

Previous work using the error function model for instantaneous SSH has used largely interactive techniques to tweak parameters until a satisfactory fit is achieved. Because 
of the large quantity of data available for the Southern Ocean and the necessity of performing statistical tests on the data to assess the magnitude of the errors, for this study the data processing has been entirely automated.

First, initial guesses are obtained for the six parameters, $a_{i}$, representing position, width, and height of the SAF and PF as a function of time along each ground track. The appendix shows that the standard deviation of the SSH (shown in Figure 2.4) may be rescaled to serve as a reasonable proxy for mean geostrophic velocity; in turn, this is used to derive a first guess of the velocity field. Using the variance maxima (chosen to be within about $5^{\circ}$ of the historic frontal locations) as rough constraints on mean jet positions, two velocity maxima are automatically selected to represent the SAF and the PF, and the corresponding jet parameters are identified.

These initial parameter guesses were iteratively improved by alternating application of the Levenberg-Marquardt procedure, a nonlinear least squares fitting algorithm [Press et al., 1986] with automatic selection of new parameters based on the revised SSH estimates. ${ }^{2}$ By locally linearizing, the Levenberg-Marquardt scheme determines an optimal direction in parameter space to readjust the $a_{i}$ in order to minimize the merit function:

$$
\chi^{2}(t)=\sum_{x}\left[h^{\prime}(x, t)+\bar{h}(x)-h_{\text {model }}\left(x, a_{i}, d\right)\right]^{2},
$$

where $h^{\prime}$ is again the measured residual SSH, $\bar{h}$ is the reconstructed mean SSH, and $h_{\text {model }}$ is the instantaneous SSH. The value $d$ is a constant offset which accounts for

\footnotetext{
${ }^{2}$ An alternative method would be simulated annealing, in which a large range of parameter space is sampled by randomly varying each of the $7 \times N$ parameters in turn. When a smaller value of $\chi^{2}$ is found and sometimes when a larger value is found, the parameters are reset. As the fit converges, the range in which the parameters may be randomly adjusted is slowly narrowed, analogous to cooling the temperature to reduce molecular motions in the physical annealing process. The algorithms necessary for simulated annealing are conceptually simple, and in its theoretical limits simulated annealing guarantees finding the best possible minimization of the function $\chi^{2}$. However, as Barth and Wunsch [1990] found, such computations are frequently extremely costly. For this particular problem, each orbit requires many hours of CPU time on a Sparc I workstation to process. With available computer resources, the method was prohibitively slow; instead the more direct computational procedures described in the text were implemented.
} 


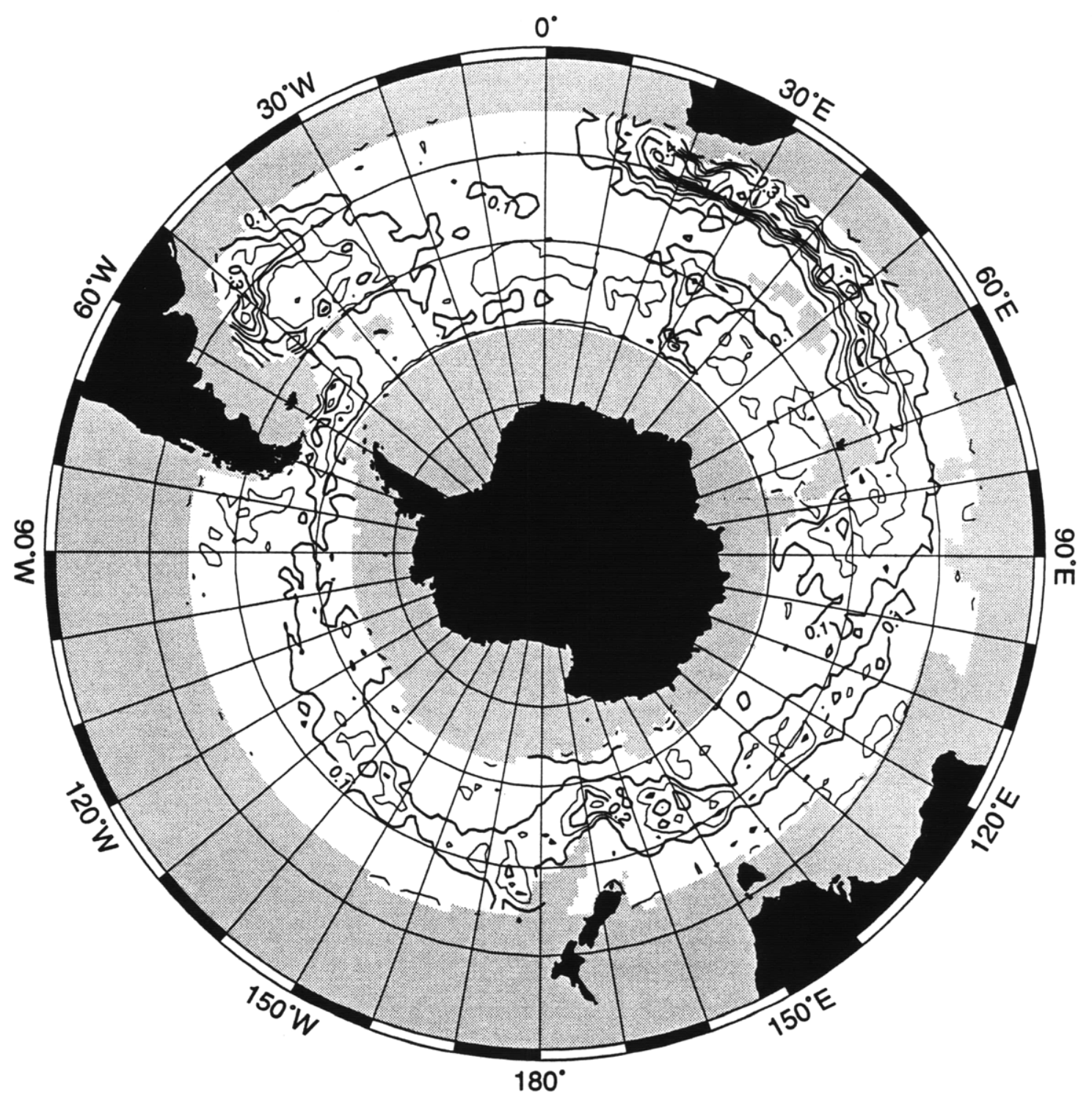

Figure 2.4: Root mean squared SSH variability (in meters) estimated from altimeter data and objective mapped using the same decorrelation function as for the mean $\mathrm{SSH}$ (discussed in section 2.4). Gray areas indicate regions where the error estimate exceeds $0.07 \mathrm{~m}$ or where no data is available. 
the residual orbit error and has no dynamical significance. The time variable, $t$, represents the satellite pass and has a maximum, $N$, which varies between 35 and 60 . To improve the stability of the fitting process, the standard merit function, $\chi^{2}$, was slightly modified to loosely anchor the parameters:

$$
\tilde{\chi}^{2}(t)=\chi^{2}(t)+\sum_{i=1}^{6}\left\{b_{i} \cosh \left[c\left(a_{i}(t)-\bar{a}_{i}\right)\right]\right\}^{2},
$$

where $b_{i}$ and $c$ may be arbitrarily fixed to keep the parameters, $a_{i}(t)$ within a physically plausible range of $\bar{a}_{i}$. The hyperbolic cosine function is selected because it has little effect on a parameter $a_{i}$ if it is near $\bar{a}_{i}$, but very dramatically changes $a_{i}$ if it strays out of range. Between applications of the Levenberg-Marquardt technique, the parameters are reestimated by adding the revised mean SSH to the residual heights and selecting features corresponding to large SSH differences. The underlying assumption in estimating jet parameters is that the location of the jet is the critical parameter: if the correct bump in the SSH can be identified, then more precise estimates of the location, width, and height difference may be found by tuning. Residual bumps and dips in the SSH are interpreted as rings. Conditions for temporal and between track continuity are not included in the model; long-period, large-scale structure in the final results is therefore a rough indication of the overall consistency of this method. In locations where a third ACC front exists at a detectable latitude, the analysis may occasionally misidentify it as either the SAF or PF, though this does not appear to be a severe problem: in about $98 \%$ of adjacent track pairs, the mean is consistent with the presence of two continuous jets, though in some locations the jets may nearly merge.

\subsection{Error Estimation}

In order to estimate the error in the reconstructed mean SSH, Monte Carlo simulation was performed for the 28 tracks identified in Figure 2.1. (Press et al. [1986] outline the basic principles underlying this method.) Since both the accuracy (or bias) and 
the precision (or repeatability) of the nonlinear fitting are of concern, two different Monte Carlo schemes were developed. Both types of Monte Carlo simulation share common procedures: Begin with a 34-month time series of up to $N=62 \mathrm{SSH}$ profiles along one of the ground tracks; estimate a time series of $7 \times N$ jet parameters and a mean SSH for the data; now add random noise to the original data to create multiple new data sets. (Ten to twenty sets of data with random noise were sufficient to produce error bars accurate to at least one significant digit.) Process each of these new data sets like the original data set to generate new estimates of the mean SSH and the $7 \times N$ parameters. Then compute the differences between the results from the original data and from the noise-added data to estimate error bars for the mean and for each of the parameters.

\subsubsection{Assessing Bias From "Perfect" Data}

How successfully did the nonlinear fitting procedure reconstruct a known mean with perfect data? To determine if there is a measurable bias in the procedures, a time series of six estimated parameters that define the instantaneous SSH was used to create a noiseless artificial data set from which the mean SSH was removed to produce "perfect" residual heights. Since the correct parameters are known, any systematic differences between the correct answers and simulated results will represent potential biases in the results. The resulting mean bias of $0.02 \mathrm{~m}$ was small compared with its standard deviation of $0.06 \mathrm{~m}$.

In real data, eddies and rings along with instrument noise will lead to additional random error in the estimated mean height. To investigate the relative magnitudes of the bias compared with the error due to mesoscale noise, Monte Carlo simulations were performed by first adding noise to the synthetic data. The misfit between the modeled SSH and the reconstructed mean plus residual height is characteristic of large eddy features which occur near the SAF and PF. (In Figure 2.2, this misfit is the difference between the dashed and solid lines.) The spectrum of this difference 
was used to set the amplitude of random noise, with a minimum level of $0.03 \mathrm{~m}$ corresponding to the background noise of the Geosat altimeter. The noise spectrum with random phases was inverted to the spatial domain and low-pass filtered in the same way as the data. The addition of eddies did not substantially change the results of the no-eddy case: the mean bias, $0.03 \mathrm{~m}$, was small compared with the standard deviation.

\subsubsection{Assessing Repeatability (Precision)}

Precision, or repeatability, is another measure of the success of a fitting scheme. To test precision, a second type of Monte Carlo procedure was employed by perturbing the original raw data with noise designed to best represent two types of measurement errors. Random white noise with about $0.03 \mathrm{~m}$ amplitude was combined with noise computed from the spectrum of the unused data corrections for the solid earth tide, ionospheric and wet tropospheric delays, and the EM bias, as shown in Figure 2.5. The EM bias correction, which dominated the spectrum, was red, with amplitudes of 0.1 to $0.2 \mathrm{~m}$ for long wavelengths, and a $0.03 \mathrm{~m}$ minimum amplitude was imposed. (The long-wave amplitudes of this spectrum are also large enough to account for Chelton's [1988] estimated errors in the corrections applied to the data.) Noise generated by inverse Fourier transforming the amplitude with random phase was added to the raw data, and the resulting data were processed following the procedure outlined in section 2.2.

The mean root mean square (RMS) difference for all 28 ground tracks tested was $0.077 \mathrm{~m}$, which represents about a $12 \%$ error in the mean SSH. Figure 2.6 shows the reconstructed mean SSH for one sample track, d001, along with the RMS error computed based on 20 simulations with noisy data. The RMS error and the reciprocal square root of the number of cycles, $N^{-\frac{1}{2}}$, have a correlation coefficient of 0.48 , which exceeds the $95 \%$ significance level. A linear regression of this relationship was used 


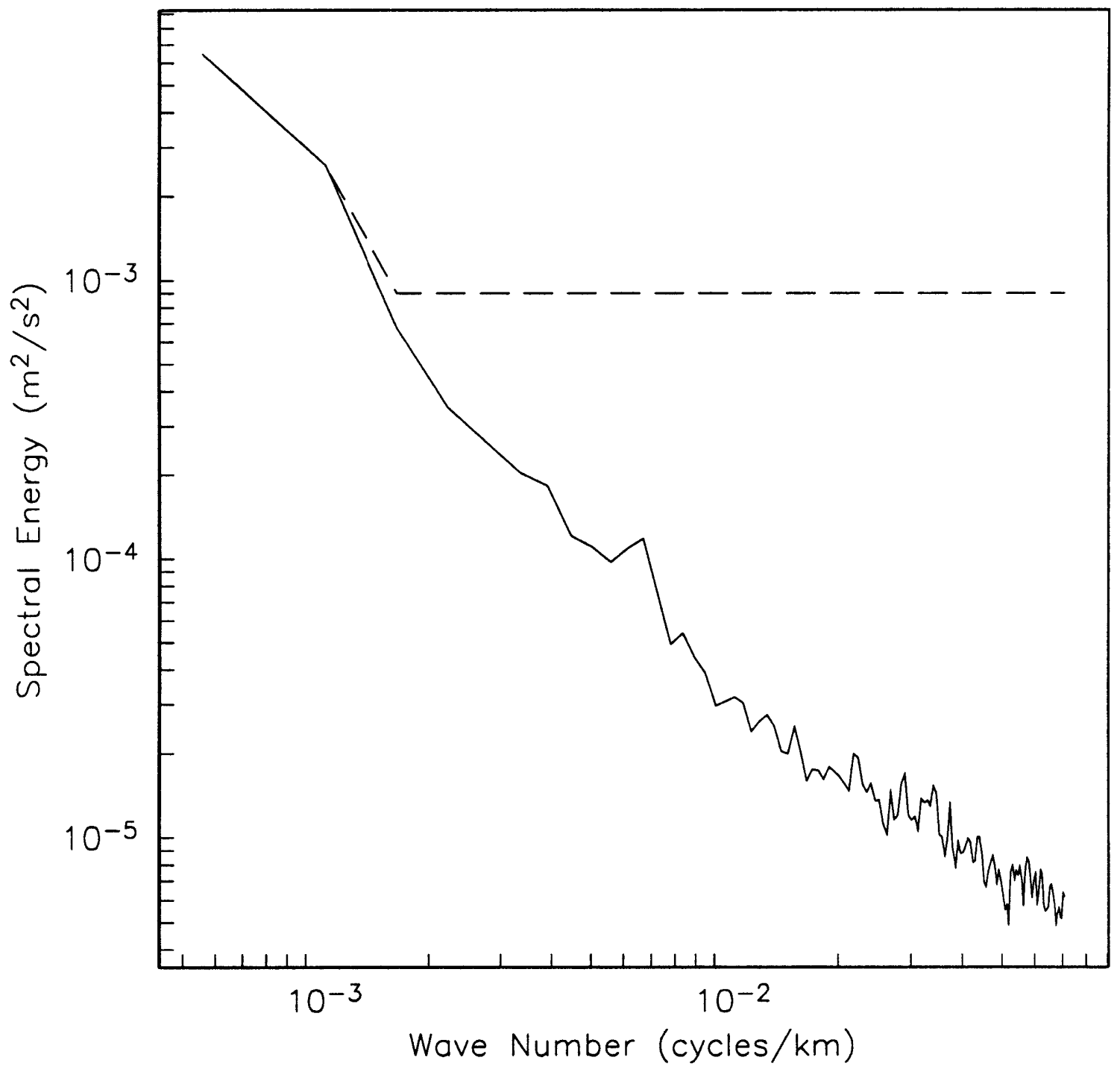

Figure 2.5: Spectrum of data corrections (solid line) for track d001. For the Monte Carlo simulations, a $0.03 \mathrm{~m}$ minimum level of white noise is added (dashed line.) 


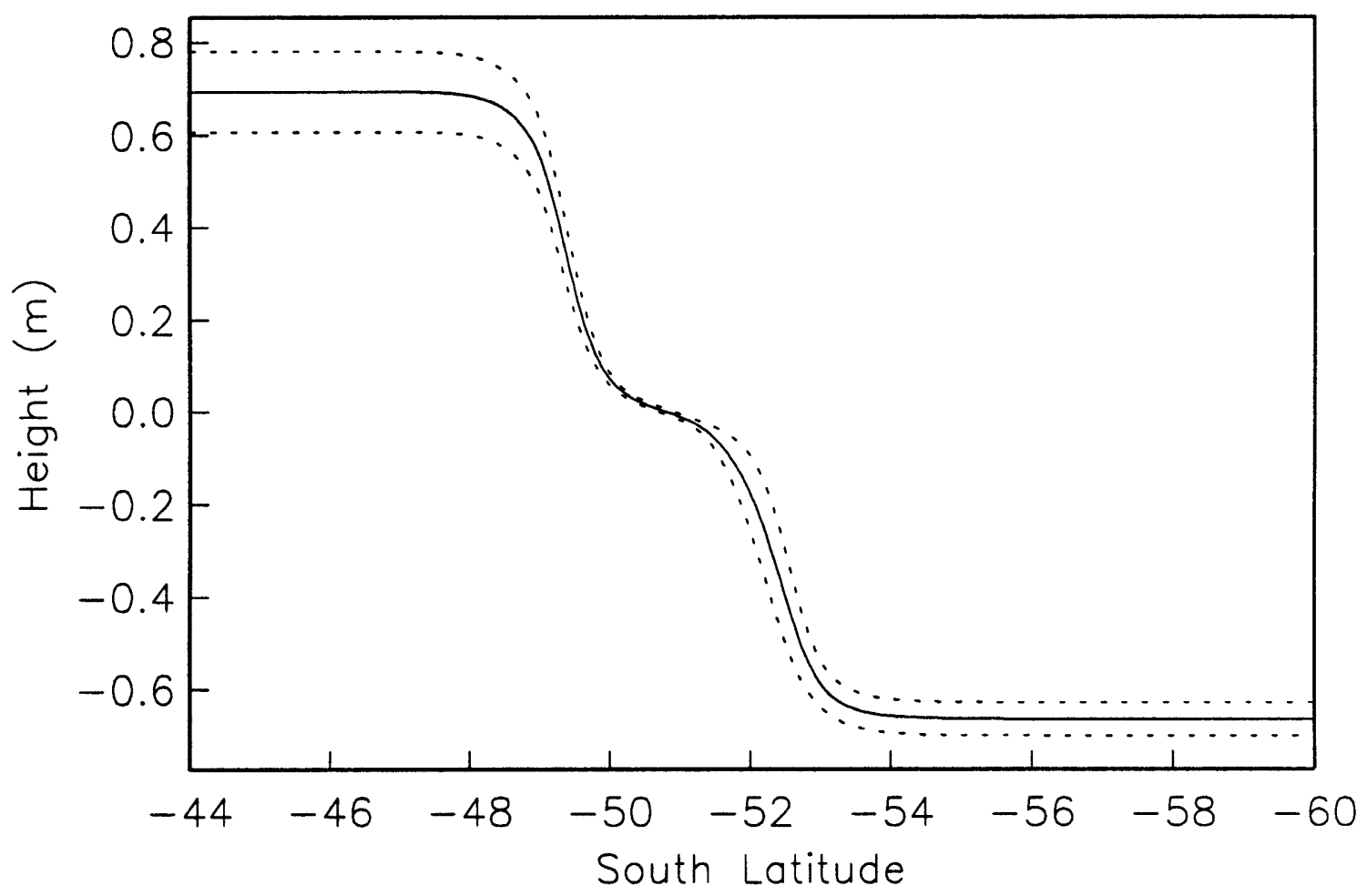

Figure 2.6: Mean SSH for track d001 (solid) and 1- $\sigma$ RMS errors (dashed) estimated from Monte Carlo simulation. The error is smallest at the center because the separation between the jets is used as a fixed reference point. 
to estimate the SSH error for ground tracks where Monte Carlo simulations were not performed.

Errors were also computed for each of the six jet parameters. (The constant offset is considered arbitrary.) They are listed in Table 2.2. All of the distributions are centered at zero, indicating no substantial bias in the parameters, but the distributions have long tails; thus a double exponential probability distribution may be more appropriate than the usual Gaussian normal distribution to characterize errors in the parameters, and statistically robust techniques should be employed to interpret these parameters. Therefore the mean absolute value of the error (the L1 norm) rather than the standard deviation was used for some analyses. Table 2.2 also shows that the error estimates computed from the diagonals of the linearized covariance matrix for the Levenberg-Marquardt nonlinear fit are in most cases about the same order of magnitude as the error bars from the Monte Carlo simulation, but occasionally the nonlinear fit generates excessively large error estimates. The mean Monte Carlo simulation results, presented at the end of Table 2.2, provide a more consistently reliable error estimate.

Based on the Monte Carlo simulation results, the signal-to-noise ratio appears large enough to permit consideration of time-dependent characteristics of the ACC frontal structure. Figure 2.7 shows a time series for one subtrack of the total height difference across the combined SAF and PF along with RMS errors at each point. While the error bars are occasionally quite large, low-frequency fluctuations have an amplitude of about $0.15 \mathrm{~m}$, which is significantly greater than most of the estimated error bars. The standard deviation of time-dependent fluctuations throughout the Southern Ocean, $7 \%$ of the mean, is comparable to the $8 \%$ fluctuations in total transport observed by Whitworth and Peterson [1985] in Drake Passage. In their transport data record, as in the height results presented here, the maximum amplitude of the fluctuations is $16 \%$ of the mean, suggesting that the magnitude of variability observed in Drake Passage may be representative of the entire circumpolar system. Later dis- 


\begin{tabular}{|c|c|c|c|c|c|}
\hline & & Parameter & $\begin{array}{c}\text { Mean } \\
\text { Absolute } \\
\text { Value of } \\
\text { Error }\end{array}$ & $\begin{array}{c}\text { Error } \\
\text { From } \\
\text { Covariance } \\
\text { Matrix }\end{array}$ & $\begin{array}{l}\text { Mean } \\
\text { Value }\end{array}$ \\
\hline $\mathrm{a} 000$ & $\overline{\mathrm{SAF}}$ & $a_{1}$ height & 0.13 & 0.15 & 0.42 \\
\hline & & $a_{2}$ position & 0.88 & 0.49 & -48.73 \\
\hline & & $a_{3}$ width & 0.25 & 0.26 & 0.42 \\
\hline & PF & $a_{1}$ height & 0.15 & 0.17 & 0.72 \\
\hline & & $a_{2}$ position & 0.24 & 2.08 & -50.67 \\
\hline & & $a_{3}$ width & 0.16 & 0.09 & 0.42 \\
\hline 2008 & $\overline{\text { SAF }}$ & $a_{1}$ height & 0.12 & 0.16 & 0.81 \\
\hline & & $a_{2}$ position & 0.17 & 0.19 & -52.73 \\
\hline & & $a_{3}$ width & 0.12 & 0.08 & 0.54 \\
\hline & $\mathrm{PF}$ & $a_{1}$ height & 0.11 & 0.17 & 0.78 \\
\hline & & $a_{2}$ position & 0.66 & 0.26 & -55.47 \\
\hline & & $a_{3}$ width & 0.23 & 0.21 & 0.31 \\
\hline $\mathrm{d} 000$ & $\overline{\text { SAF }}$ & $a_{1}$ height & 0.33 & 0.21 & 0.71 \\
\hline & & $a_{2}$ position & 0.84 & 8.96 & -55.40 \\
\hline & & $a_{3}$ width & 0.25 & 14.12 & 0.27 \\
\hline & $\mathrm{PF}$ & $a_{1}$ height & 0.32 & 0.25 & 0.78 \\
\hline & & $a_{2}$ position & 0.72 & 1.41 & -56.98 \\
\hline & & $a_{3}$ width & 0.26 & 0.62 & 0.30 \\
\hline $\mathrm{d} 008$ & SAF & $a_{1}$ height & 0.17 & 0.19 & 0.62 \\
\hline & & $a_{2}$ position & 0.38 & 0.88 & -43.05 \\
\hline & & $a_{3}$ width & 0.26 & 0.14 & 0.56 \\
\hline & $\mathrm{PF}$ & $a_{1}$ height & 0.15 & 0.17 & 0.78 \\
\hline & & $a_{2}$ position & 0.36 & 0.60 & -44.97 \\
\hline & & $a_{3}$ width & 0.19 & 0.25 & 0.46 \\
\hline Mean & SAF & $a_{1}$ height & 0.17 & 0.18 & 0.68 \\
\hline & & $a_{2}$ position & 0.54 & 4.16 & -49.76 \\
\hline & & $a_{3}$ width & 0.17 & 1.06 & 0.40 \\
\hline & $\mathrm{PF}$ & $a_{1}$ height & 0.15 & 0.19 & 0.60 \\
\hline & & $a_{2}$ position & 0.53 & 0.69 & -53.04 \\
\hline & & $a_{3}$ width & 0.17 & 0.33 & 0.39 \\
\hline
\end{tabular}

Table 2.2: Mean Values and Results of Monte Carlo Simulation for Gaussian Jet Parameters for SAF and PF for Four Tracks. The mean absolute value of the error (the L1 norm) is shown along with the error estimated based on the covariance matrix computed for the Levenberg-Marquardt fit and the mean values of each of the parameters. Here, $a_{1}$ heights are in meters; $a_{2}$ positions and $a_{3}$ widths are in degrees latitude. 


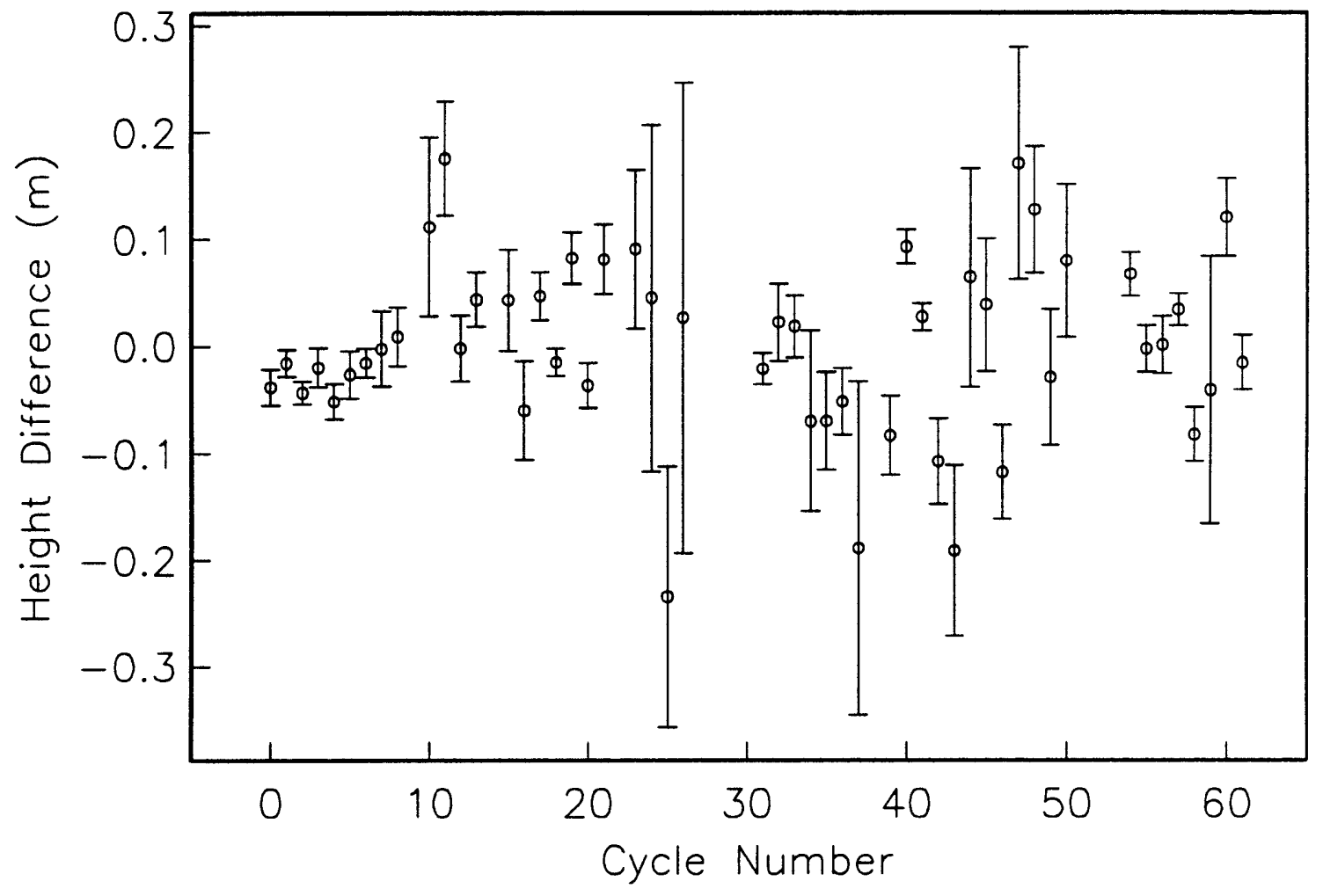

Figure 2.7: Time series of total height difference across the SAF and PF, with (L1 norm) error computed based on 20 Monte Carlo simulations for track d001. 
cussions of the altimeter-derived mean SSH data will examine the time dependence and the dynamics controlling the ACC in greater detail, but the remainder of this chapter focuses on the mean ACC.

\subsection{Mapping Regional Results}

\subsubsection{Mean Sea Surface Height}

Mean heights were estimated for all of the track segments shown in Figure 2.1 and were objective mapped using the method outlined by Bretherton et al. [1976]. Covariance functions needed to objective map the height fields were obtained by least squares fitting of data covariances to a function of the form

$$
c_{i, j}=A \exp \left[-\left(\frac{\Delta x_{i j}^{2}}{B_{1}^{2}}+\frac{\Delta y_{i j}^{2}}{B_{2}^{2}}\right)\right]+C_{1} \delta_{i j}+C_{2} \delta_{k l}
$$

where $A$ is the amplitude corresponding to the zero-lag covariance. Because the data variance depends strongly on whether a point is within or outside a jet, $A$ is largest where the variance is largest, along the core of the ACC, and attenuates with distance from the jets according to the empirical relation:

$$
A=0.1 \exp \left(-\frac{\Delta y^{2}}{\left(1.8^{\circ}\right)^{2}}\right)+0.02 \mathrm{~m}^{2}
$$

where $\Delta y$ is the difference in degrees latitude between the SAF-PF midpoint and the objective map grid point. $B_{1}$ and $B_{2}$ are zonal and meridional decorrelation length scales. Using adjacent ground tracks, the zonal scale, $B_{1}$, was estimated to be about $200 \mathrm{~km}$. The along-track length scale is about $160 \mathrm{~km}$. Combining this information with the zonal length scales to project into the meridional direction, the meridional length scale, $B_{2}$, is estimated to be $100 \mathrm{~km}$. These distances are less than half of the 450-km zonal and 350-km meridional length scales used by Olbers et al. [1992] for objective mapping temporally and spatially coarser hydrographic data and indicate that the maps produced from altimeter data should retain greater spatial structure. 
$C_{1}$ and $C_{2}$ represent the error variance, and the subscripts $i$ and $j$ refer to individual data points (regardless of subtrack), while the subscripts $k$ and $l$ refer to different satellite ground tracks. The quantity $C_{1}+C_{2}$ is estimated from the data to be approximately $0.012 \mathrm{~m}^{2}$. The value of $C_{2}$ estimated from the Monte Carlo simulation is $\left(7.7 \times 10^{-2} \mathrm{~m}\right)^{2}$ or about $60 \times 10^{-4} \mathrm{~m}^{2}$. Thus $C_{1}$ is also set to $60 \times 10^{-4} \mathrm{~m}^{2}$. Note that $C_{2}$ is nonzero because errors in the mean are correlated from point to point along the satellite subtracks though not between satellite tracks. $C_{1}$ accounts for the uncorrelated error at each measurement point.

To eliminate residual orbit error between different ground tracks, heights along each ground track are adjusted by an additive constant, which is determined by minimizing ascending and descending track crossover differences in a least squares sense; this constant is typically smaller than $0.1 \mathrm{~m}$. The resulting mean SSH field, mapped with $1^{\circ}$ resolution, is shown in Figure 2.8.

\subsection{Discussion}

The mean SSH based on the altimeter data (Figure 2.8) shows that the height gradient associated with the ACC occurs in a relatively narrow region. This clearly contrasts with the broader flow patterns indicated by dynamic topography computed from Olbers et al.'s [1992] gridded data (Figure 2.9). No sharp position changes between adjacent tracks or localized error increases exist to suggest discontinuities in the ACC. Although the two jets are not clearly discernible in Figure 2.8 because of the large geographic domain shown and coarse $1^{\circ}$ gridding of the map, the ACC is consistently modeled as two sharp eastward jets throughout the Southern Ocean.

\subsubsection{Jet Statistics}

The Gaussian model parameters provide a simple means to characterize the structure of the ACC jets and to track changes in the ACC. Mean parameter values are 


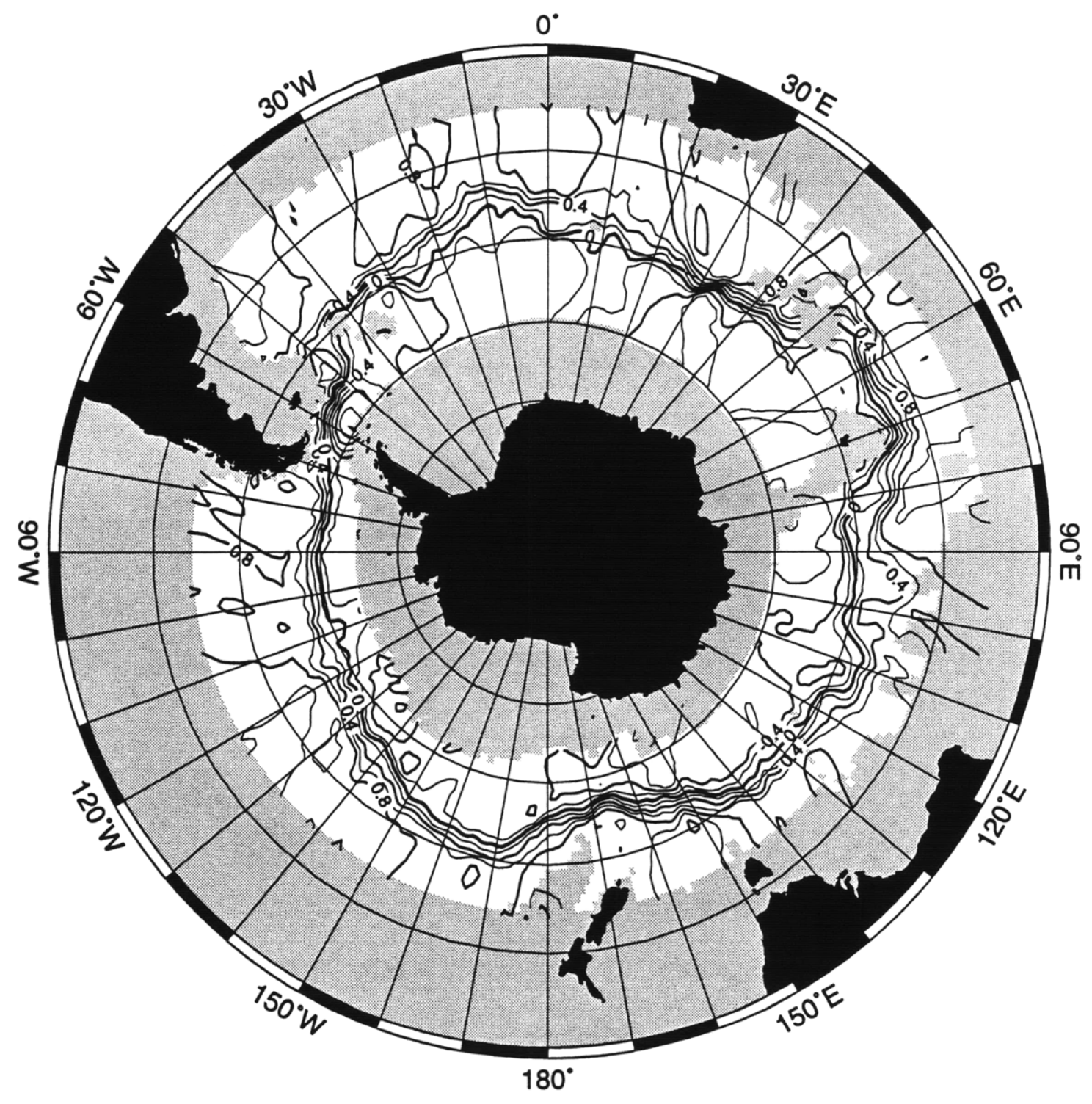

Figure 2.8: Objective mapped reconstructed mean SSH, contoured at 0.2-m intervals. The $0-\mathrm{m}$ height contour separates the SAF (positive heights) from the PF (negative heights). Regions with no data or height errors greater than $0.05 \mathrm{~m}$ are gray, and contours are not shown when the error exceeds $0.1 \mathrm{~m}$. Typical errors are between 0.04 and $0.05 \mathrm{~m}$. 


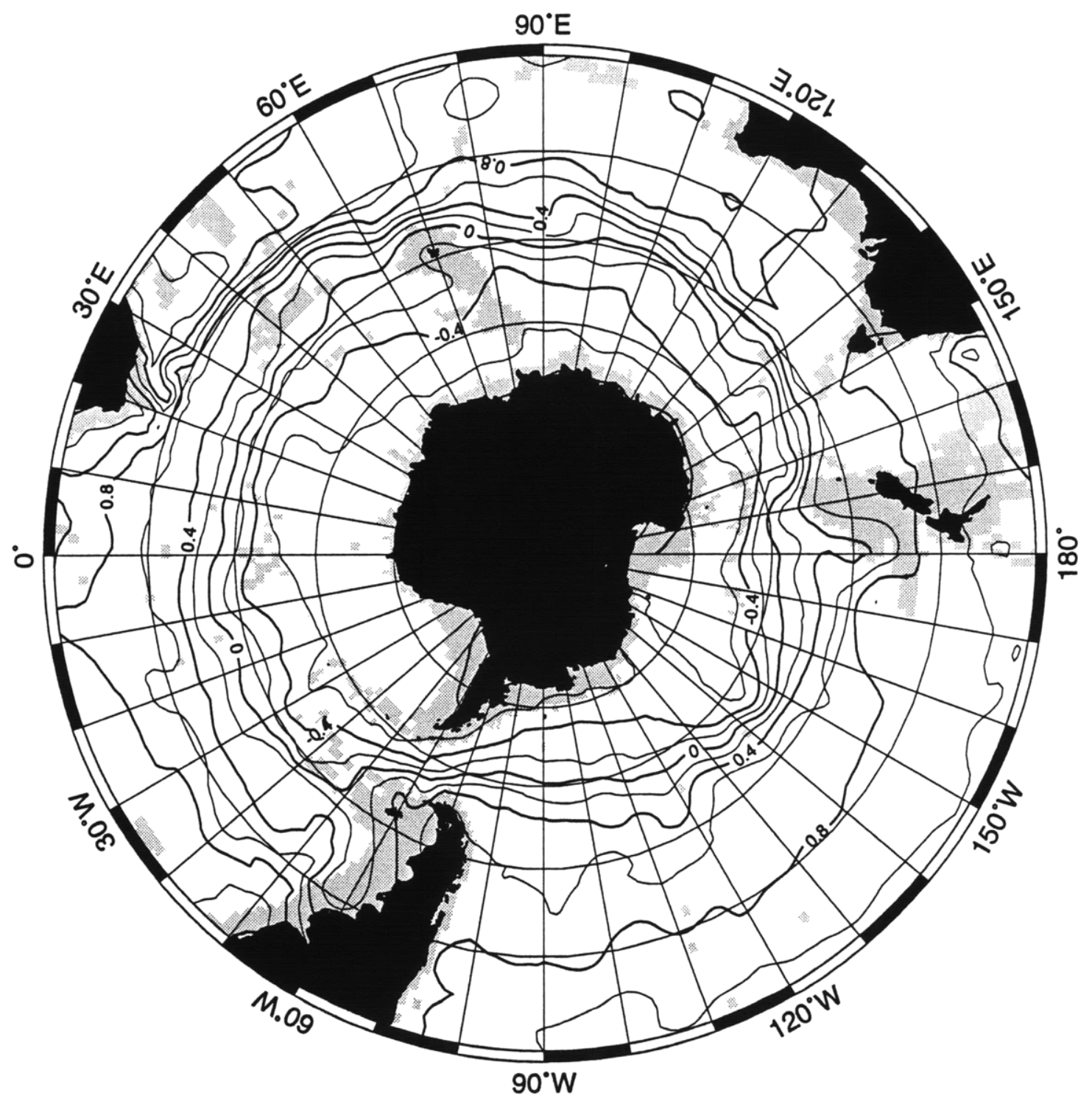

Figure 2.9: Historic mean dynamic height (in meters) from Olbers et al. [1992]. Dynamic topography is referenced to $2500 \mathrm{~m}$, and has been adjusted by an arbitrary constant to simplify comparison with the reconstructed SSH shown in Figure 2.8. 
summarized in Table 2.2. In Figure 2.10, the mean latitudes of the SAF and PF, objectively mapped using data from ascending and descending tracks, indicate that the jets are closely steered by bathymetry, in agreement with analyses of hydrographic data [GMB78]. The response of the SAF and PF to specific topographic features, such as the Eltanin and Udintsev Fracture Zones around $130^{\circ} \mathrm{W}$, will be discussed in the geographic tour of the Southern Ocean later in this section. The meridional separation between the fronts varies from $55 \mathrm{~km}$ to $900 \mathrm{~km}$, with a mean separation of $310 \mathrm{~km}$. Over time both jets meander; however, the mean absolute value of the position fluctuations (the $\mathrm{L} 1$ norm) is $0.47^{\circ}$, which is less than the error estimates from Table $2.2\left(0.54^{\circ}\right.$ and $\left.0.53^{\circ}\right)$, indicating that the signal-to-noise ratio is small and detailed information about the global time series may be difficult to extract.

The mean height difference across the two fronts is $1.3 \mathrm{~m}$. This is slightly larger than the $1.1 \mathrm{~m}$ dynamic height difference found by referencing historic data to 2500 or $3000 \mathrm{~m}$ [Gordon et al., 1982; Olbers et al., 1992]. Figure 2.11 shows how the mean height difference varies around the Southern Ocean. Though the total height difference shows substantial scatter, consistent with the error estimates in Table 2.2, one striking feature is its apparent tendency to oscillate between two values, a minimum around $1.1 \mathrm{~m}$ and a larger value around $1.5 \mathrm{~m}$. The $1.1-\mathrm{m}$ difference may represent the circumpolar flow, and the larger height differences correspond to regions where, for example, parts of the recirculating subtropical gyre in the Indian Ocean (between $20^{\circ}$ and $80^{\circ} \mathrm{E}$ ) or the Weddell Sea gyre (between $70^{\circ}$ and $40^{\circ} \mathrm{W}$ ) join the circumpolar flow. The height differences across the jets are correlated with the SSH variance with a coefficient of 0.60 , which is significant at the $99.9 \%$ level. Simulations using artificial data indicate that this correlation is not an artifact of the data processing. Maps by Johnson et al. [1992] and Morrow et al. [1992] suggest that regions of high height variance are associated with increased Reynolds stress terms; therefore changes in the height differences may be linked to changes in the importance 


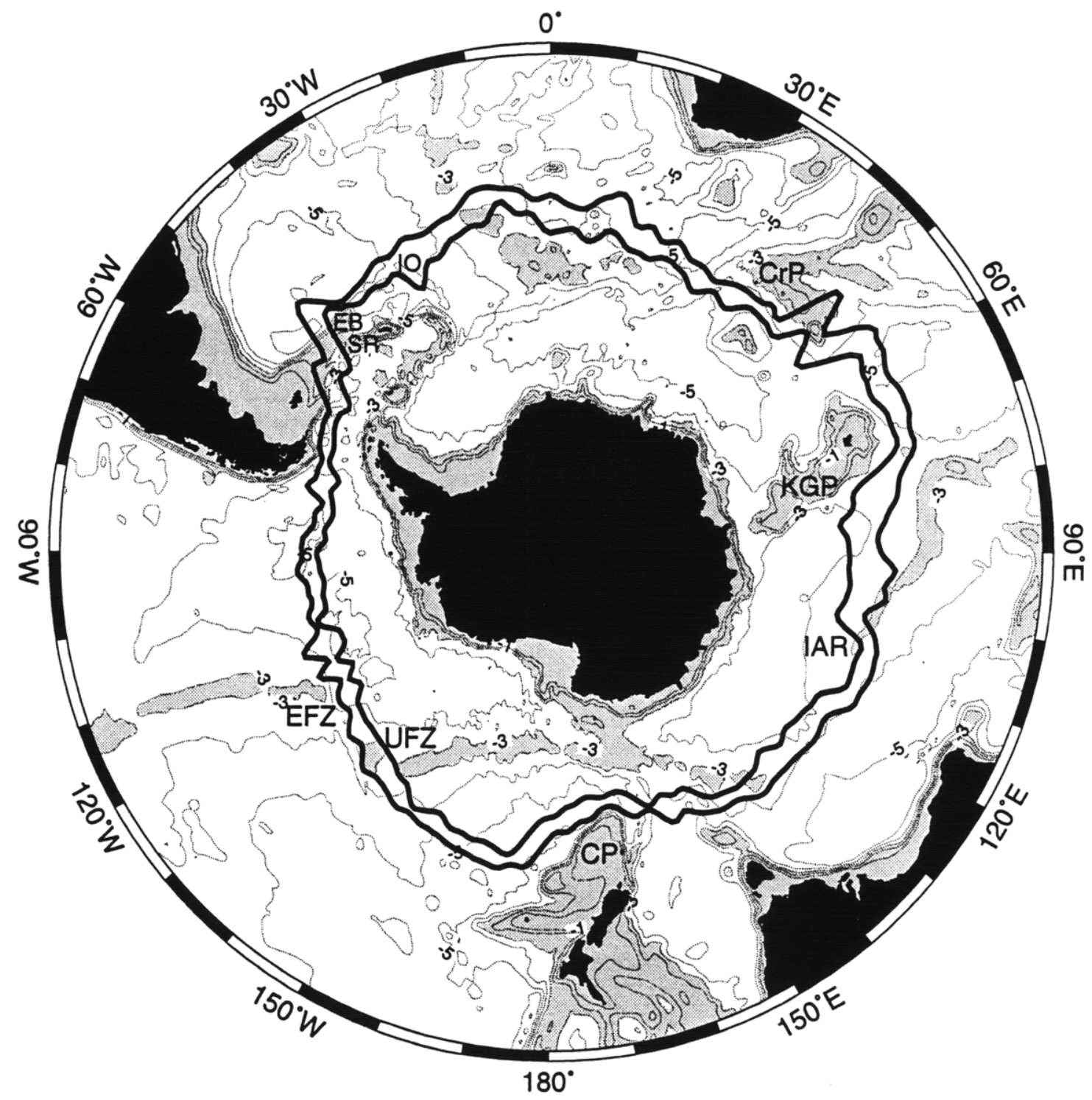

Figure 2.10: Bathymetry in kilometers from gridded 5 minute data smoothed over 100 $\mathrm{km}$ lengthscales. Regions shallower than $3 \mathrm{~km}$ are shaded gray. Indicated features are Indian-Antarctic Ridge (IAR), Campbell Plateau (CP), Udintsev Fracture Zone (UFZ), Eltanin Fracture Zone (EFZ), Scotia Ridge (SR), Ewing Bank (EB), Islas Orcadas Rise (IO), Crozet Plateau (CrP), Kerguelen-Gaussberg Plateau (KGP). The heavy shallow lines represent the mean paths of the SAF and PF. 


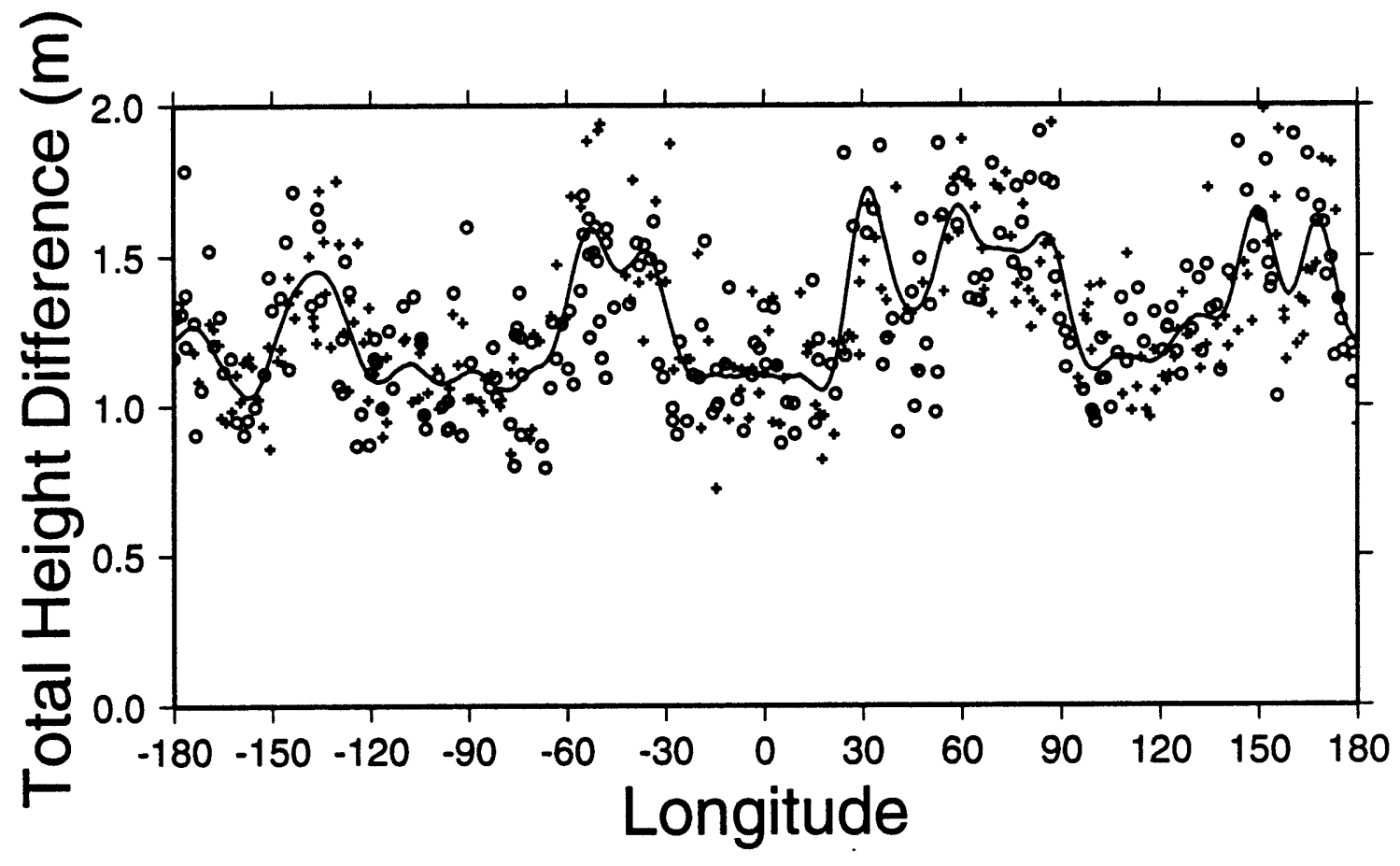

Figure 2.11: Mean height across both the SAF and PF as a function of longitude for ascending tracks (circles) and descending tracks (pluses). The solid line represents the objectively mapped height differences. 
of the ageostrophic components of the momentum balance rather than to changes in transport.

Figure 2.12 shows histograms of all heights within the Southern Ocean. The most common heights represent the relatively flat regions outside of the ACC. The strong height gradients across the ACC jets correspond to less frequently occurring heights. The dynamic height from Olbers et al.'s [1992] gridded atlas data (Figure 2.12a) does not distinguish two separate ACC jets because the probability decreases gradually near zero, the height value separating the jets. The distribution of mean SSH for all the satellite tracks with no crossover corrections added (Figure $2.12 \mathrm{~b}$ ) suggests two distinct jets separated by the zero height contour. The addition of a crossover correction (Figure 2.12c) tends to blur the distinction between the fronts and suggests that the height contour separating the SAF and the PF may vary geographically from the nominal zero value.

The mean value for the width parameter, $a_{3}$, for both the SAF and the PF is $0.40^{\circ}$ latitude ( $54 \mathrm{~km}$ in the along-track direction or $44 \mathrm{~km}$ in the meridional direction). Since the standard deviations of the jet positions are substantially larger than their widths (Table 2.2), the mean should be reconstructed without significant bias, based on Qiu's [1992] tests and the Monte Carlo error simulations.

The spatial variations of the frontal widths are plotted in Figure 2.13. Although the along-track width differs substantially between adjacent tracks, Figure 2.13 suggests that the widths of the fronts vary by about $20 \%$ over large spatial scales. Variations in the mean angle at which the satellite groundtracks intersect the fronts cannot explain this trend.

Two factors should control the width of the ACC jets. First, the baroclinic Rossby radius, $L_{r}$, defines a horizontal length-scale for stable mesoscale features. For an ocean of depth $D$ and constant Brunt-Väisälä frequency, $N, L_{r}$ is $N D / f$. Estimates of $L_{r}$ using varying $N$ from Olbers et al.'s [1992] gridded data indicate that 


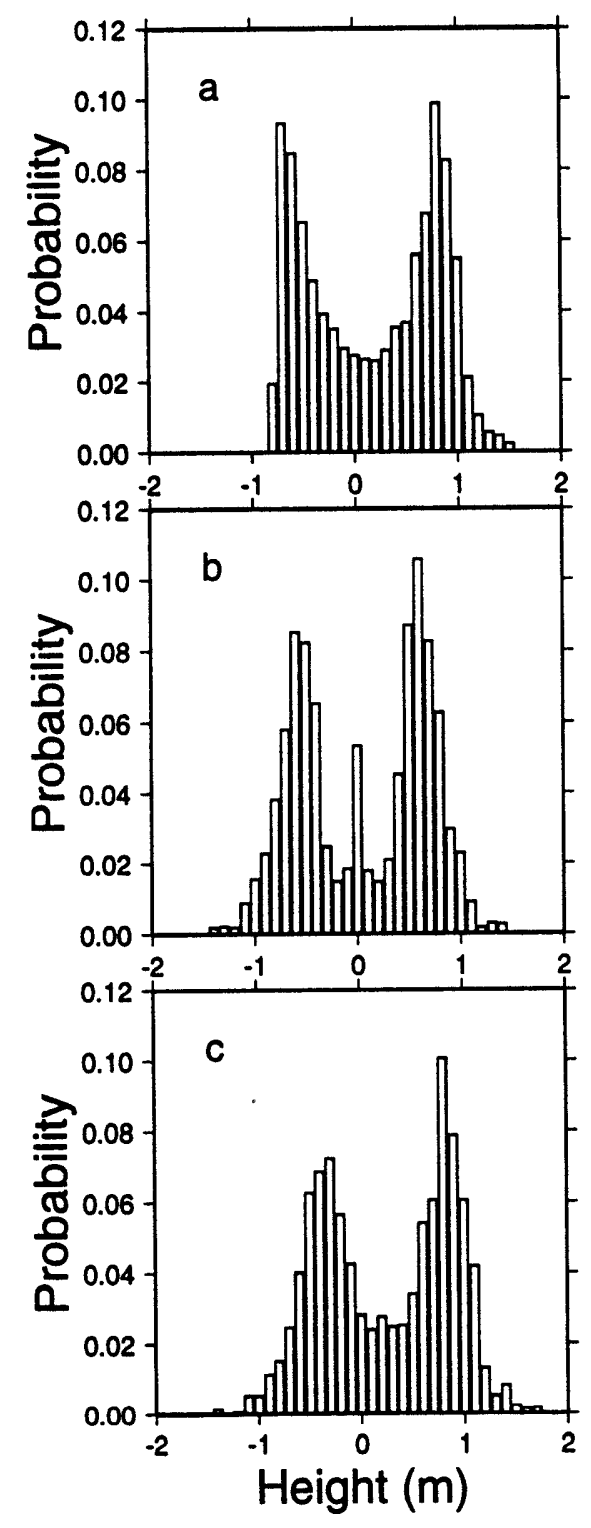

Figure 2.12: Histograms showing distribution of (a) dynamic topography in meters (from Olbers et al. [1992]'s gridded atlas data), (b) mean SSH derived from Geosat data, and (c) mean SSH from Geosat data with crossover correction to correspond to the SSH in Figure 2.8. In these histograms, the zero-height line is adjusted to fall between the SAF and the PF. 

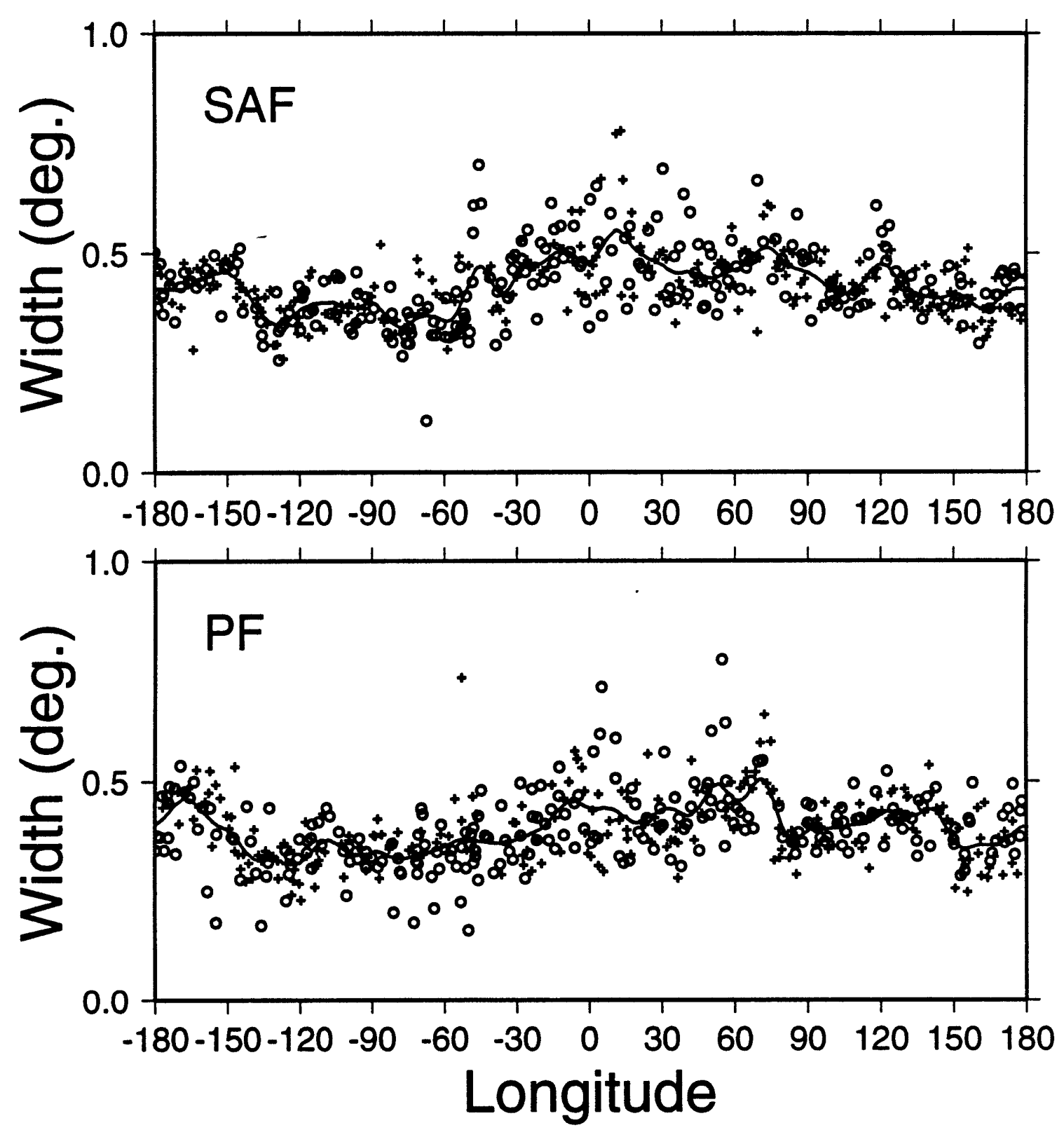

Figure 2.13: Mean width of the SAF (top) and PF (bottom) as a function of longitude. Values for ascending tracks are shown as circles; descending widths are shown as plus signs. Solid lines are determined by objectively mapping the widths. 
the dominant variation in $L_{r}$ is because of fluctuations in $f .^{3}$ Thus, as the latitudes of the SAF and PF change around the Southern Ocean, stability constraints require that the widths of the fronts adjust with $1 / f$. As the fronts flow southward from the dateline to Drake Passage (around $80^{\circ} \mathrm{W}$ ), both narrow gradually. Beyond Drake Passage, they move northward through the Scotia Ridge and broaden abruptly; the SAF is widest around $10^{\circ} \mathrm{E}$ and the $\mathrm{PF}$ widest around $60^{\circ} \mathrm{E}$, where it is also furthest north. Both fronts narrow and move southward as they continue eastward to the dateline. The correlation coefficient for width and $1 / f$ (Figure 2.14) is 0.53 for the SAF and 0.51 for the PF; both are statistically significant at the $99.9 \%$ level, and a chi-squared test for the linear regression yields a value of 0.9 , indicating a reasonable match between the estimated error bars and the statistical deviation. The correlation coefficient between $L_{\boldsymbol{r}}$ and the width (both of which are relatively noisy) is smaller, 0.25 for the two fronts combined, but still statistically significant at the $99 \%$ level.

\footnotetext{
${ }^{3}$ From Pedlosky's [1987] discussion of baroclinic modes, the baroclinic Rossby radius, $L_{r}$ may be expressed as:

$$
\frac{1}{L_{r}^{2}}=\frac{f^{2}}{D^{2} n^{2} \pi^{2}} \frac{\int_{0}^{1} \frac{\rho}{N^{2}}\left|\frac{d \phi}{d z}\right|^{2} d z}{\int_{0}^{1} \rho \phi^{2} d z}
$$

where $N$ is the Brunt-Väisälä frequency and $\phi$ represents the velocity as a function of $z$. An approximation for $L_{r}$ may be found by using the Rayleigh-Ritz method, which states that if the guess for the structure of a vertical mode $\phi$ differs from the correct estimate by $\epsilon$, then the estimate of $1 / L_{r}^{2}$ will err by no more than $\epsilon^{2}$. As a simple guess the vertical velocity structure estimated from hydrographic data is used as a first approximation of the first baroclinic mode. The quantities $N^{2}$, $\phi$, and $\rho$ are computed from gridded atlas data [Olbers et al., 1992], and functional forms are fit to the data, so that:

$$
N^{2}=n_{1}+n_{2} \exp \left(-\left|\frac{z-n_{3}}{n_{4}}\right|\right)
$$$$
\phi= \begin{cases}p_{1}+p_{2} & \text { if } z<p_{3} \\ p_{1}+p_{2} \exp \left(-\frac{z-p_{3}}{p_{4}}\right) & \text { otherwise, }\end{cases}
$$

and

$$
\rho=r_{1}+r_{2} z+r_{3} \exp \left(-\frac{z}{r_{4}}\right)
$$

where the parameters $n_{i}, p_{i}$, and $r_{i}$ are are adjusted for the data at each latitude-longitude point, and $z$, the depth, is defined to be positive.

An indicator of the baroclinic transport can be obtained by vertically integrating $\phi$ using numerical techniques to yield an equivalent depth.
} 


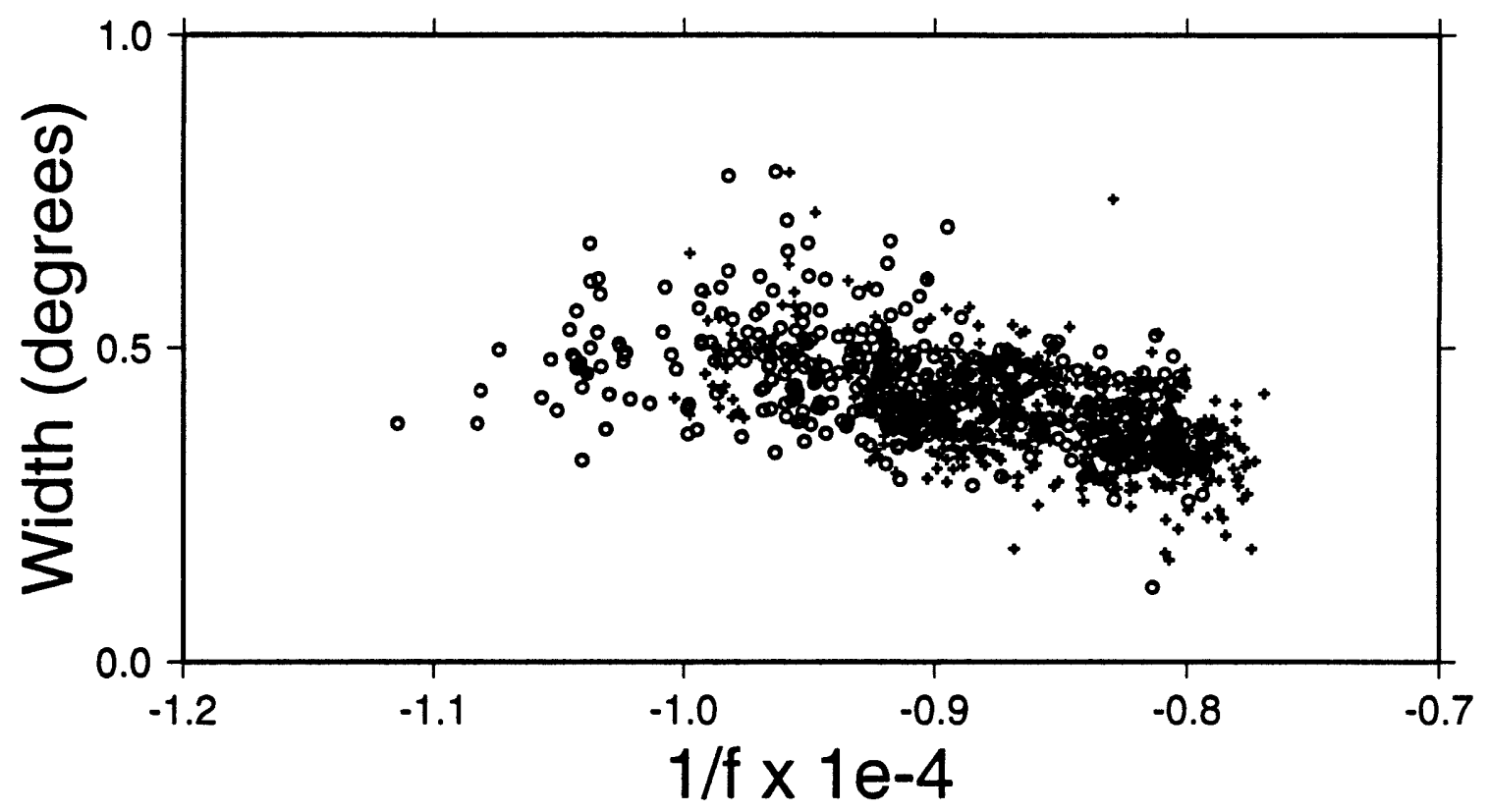

Figure 2.14: Width of the SAF (circles) and PF (pluses) versus the reciprocal of $|f|$.

Stammer and Böning [1992] found a similar relationship between $L_{r}$ and characteristic eddy length scales determined from autocovariance functions for Geosat data.

The second factor controlling the width is conservation of the total ACC transport. Since the geostrophic surface velocity of the jet is

$$
u_{0} \sim \frac{g a_{1}}{f a_{3}},
$$

the surface transport is

$$
T_{s} \sim \frac{g a_{1}}{|f|}
$$

and the total transport is

$$
T \sim \frac{g a_{1} \Phi}{|f|},
$$

where $\Phi$ represents the attenuation of the velocity with depth and may be interpreted as an equivalent depth. Although $a_{1}$ fluctuates (Figure 2.11), it does not vary systematically with latitude. Since the width, $a_{3}$, is proportional to $1 /|f|$, then $u_{s} \sim g a_{1}$, and surface transport varies with $1 /|f|$. Estimates of the vertical velocity structure of the ACC from Olbers et al.'s [1992] data suggest that $\Phi$ varies with $|f|$. 
Therefore transport varies with SSH difference, $T \sim g a_{1}$. In locations where the SAF and $\mathrm{PF}$ move southward ( $|f|$ increases), baroclinic processes may act to deepen the flow ( $\Phi$ increases) in order to conserve transport. The augmented eddy activity near topographic features which steer the ACC, indicated in Figure 2.4, suggests active instability processes may be adjusting the vertical structure.

\subsubsection{Height Variance}

The variance of the SSH measured by the altimeter (Figure 2.4) is greatest along the central axes of the ACC. In this core region between $40 \%$ and $70 \%$ of the height variance is explained by the Gaussian model for the meandering (Figure 2.15). The remaining variance represents rings and eddies separate from the jets, as well as the meandering mean flow in regions of the Southern Ocean that are not included in this model such as the Falkland Current and the Agulhas Retroflection. The percentage of height variance accounted for by the reconstructed mean is smaller in regions of increased eddy activity, which Chelton et al. [1990] noted are associated with large topographic features such as the ridge downstream of Drake Passage or the Crozet Plateau area. This suggests that topographic features may cause ring formation rather than increased jet meandering.

\subsubsection{Mean Mapped Sea Surface Height}

A few words of explanation will help the reader better interpret the mean height in Figure 2.8. The error function model used to reconstruct mean SSH applies only to the jets themselves but includes no recirculation and no large-scale structure. Thus the mean height depicted in this map should be accurate within the limits of its error bars for mesoscale variations in the jet but cannot capture basin scale height

variations. For example, this model cannot verify whether the south Pacific Ocean is $0.38 \mathrm{~m}$ higher than the south Atlantic, as suggested by Reid [1961]. In addition, 


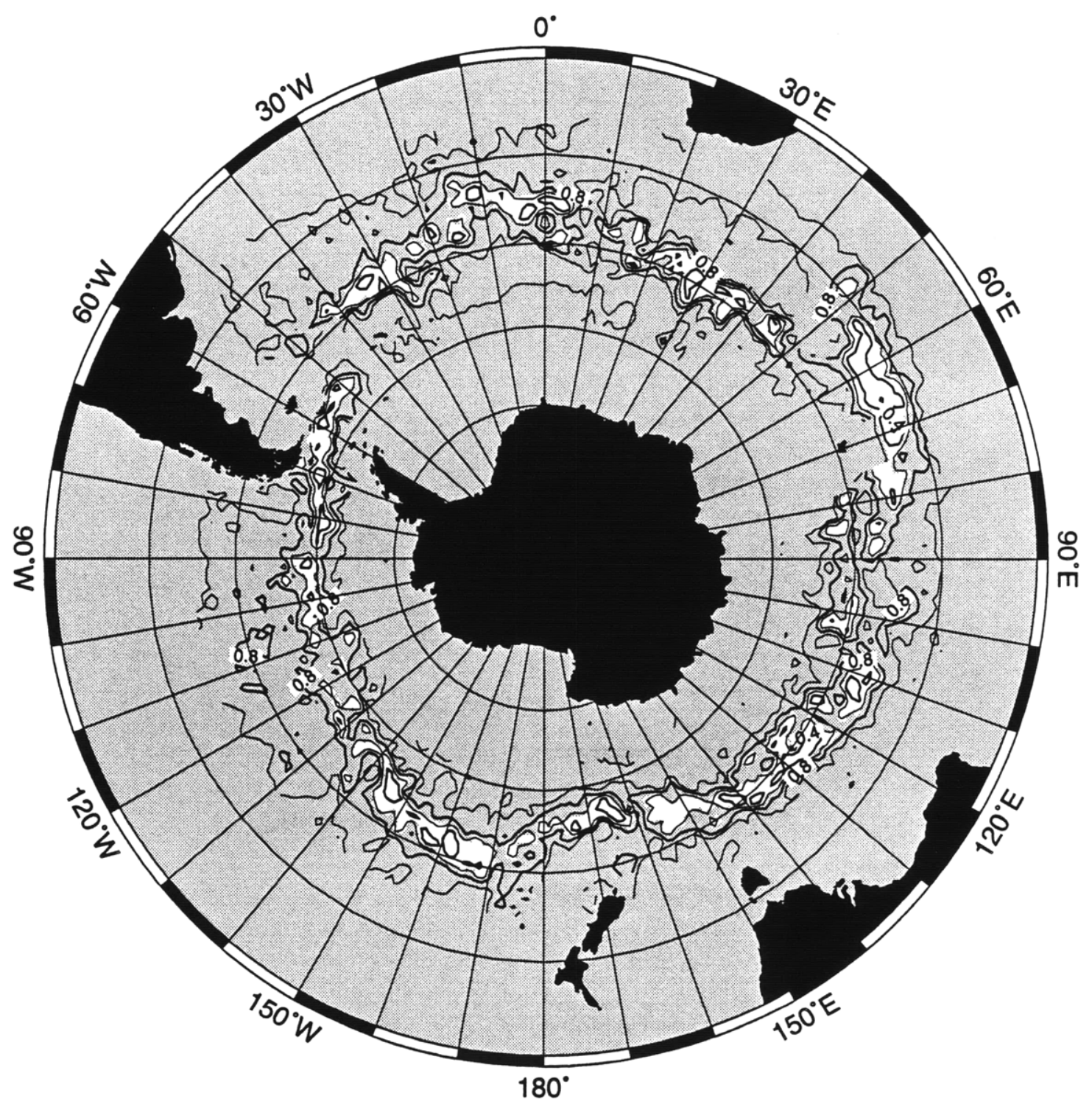

Figure 2.15: Ratio of root mean squared SSH residual variability, after modeled effects of SAF and PF have been removed, to root mean squared variability of the altimetermeasured height field. Gray areas indicate locations where no data are available or where less than $20 \%$ of the variability is explained by the meandering jet model. 
height contours which leave the mean axis in the map could identify water entering the ACC from outside the mapped region, but they could also signal ageostrophic phenomena or the effects of a recirculating flow within the mapped domain.

Several factors account for the differences between the historic hydrographic picture of the ACC as a broad eastward flow and the altimetric view of a pair of narrow jets. The most likely source of differences stems from the finer resolution provided by the altimeter, which better resolves the ACC mean structure. Timesampling considerations may also contribute to the differences. While the altimeter data represents a 30-month period in the late 1980s, the historic in situ data includes data from the 1940s through the 1980s, concentrated primarily during the Eltanin cruises of the late 1960s and early 1970s. Any substantial low-frequency shifts in ACC jet locations over the 40-year period might smear the mean ACC into a broad flow, and a local change in the structure of the ACC between the Eltanin period and the late 1980s could also account for qualitative differences in the height fields.

Finally, because the orbit corrections remove the basin scale signal, only narrow jets can be seen by the error function model. Thus if the flow were as broad as the historic data suggests and no jets existed, the algorithm could misinterpret rings or other mesoscale features as jets. However, since rings do not typically have the spatial and temporal coherence of jets, this seems unlikely to create substantial errors on the basin scale.

The following detailed examination of the altimeter mean SSH will highlight the major features of the ACC, emphasizing its response to specific bathymetric features and the differences between the altimeter picture of the ACC and the dynamic height from in situ data. The discussion follows the same organizational structure as GMB78's discussion of the Southern Ocean topography from historic data. 


\subsubsection{South of Australia, $100^{\circ}-140^{\circ} \mathrm{E}$}

South of Australia, the SAF and PF are on opposite sides of the zonal IndianAntarctic Ridge, in qualitative agreement with hydrographic measurements. The altimeter data frequently indicate the presence of eddylike features situated between the SAF and the PF. Because of difficulties in the nonlinear fitting, the data are not sufficient to assess whether this variability might represent westward counterflow over the ridge, as suggested by Callahan [1971] or transient eddies, as GMB78's analysis and Savchenko et al.'s [1978] XBT survey indicated.

\subsubsection{Tasman Sea-Campbell Plateau, $140^{\circ}-180^{\circ} \mathrm{E}$}

At $140^{\circ} \mathrm{E}$, the $\mathrm{PF}$ crosses through a gap in the Indian-Antarctic Ridge to flow adjacent to the SAF on the northern side of the ridge. At this point, the height contours pinch together slightly as the jets are closer together. The ACC flows southward to move through openings in the Macquarie Ridge-Hjort Trench system around $160^{\circ} \mathrm{E}$. In agreement with Gordon's [1972] observations, the SAF flows through an opening at about $53^{\circ} \mathrm{S}$, to the north of Macquarie Island; the PF passes just south of Macquarie Island but slightly north of the pathway identified by Gordon. Southeast of the ridge system, the two fronts converge slightly and move around Campbell Plateau south of New Zealand.

To the east of New Zealand, historic data indicates that the PF follows the northern flank of the Pacific-Antarctic ridge located at about $60^{\circ} \mathrm{S}$, while the SAF presses against the Campbell Plateau. This analysis does not place the PF as far south but instead shows both fronts moving northward in parallel around Campbell Plateau. A loss in total SSH difference at about $175^{\circ} \mathrm{W}$ may be a sign that the actual $\mathrm{PF}$ is too far south to be resolved by the altimeter, though it could also indicate the action of another dynamical process. 


\subsubsection{Southwest Pacific, $180^{\circ}-130^{\circ} \mathrm{W}$}

At $170^{\circ} \mathrm{W}$, the ACC turns eastward and moves across the abyssal plain. The PF crosses through the Udintsev Fracture Zone and the SAF crosses through the Eltanin Fracture Zone in the Pacific-Antarctic Ridge around $140^{\circ} \mathrm{W}$. At this point, the height contours converge and increase in number, suggesting a narrow and intensified transport through the ridge system. The ACC paths through the ridge gaps are readily apparent in the hydrographic data and have been interpreted as a clear indication of the coupling between surface flow and deep topography [Gordon, 1967; GMB78].

\subsubsection{Southeast Pacific, $\mathbf{1 3 0}^{\circ}-\mathbf{8 0}^{\circ} \mathrm{W}$}

East of the mid-ocean ridge, the altimeter data indicates narrow jets continuing southward toward Drake Passage, in contrast to the historic hydrographic data which shows the current rapidly broadening into a diffuse eastward flow without a clear axis [GMB78; Reid, 1986]. A slight reduction in the number of height contours across the fronts suggests that some of the transport may recirculate through the central Pacific and that the jets are less intense in the eastern Pacific than they are at the mid-Pacific ridge.

\subsubsection{Drake Passage, $80^{\circ}-55^{\circ} \mathrm{W}$}

The narrow opening through Drake Passage at $70^{\circ} \mathrm{W}$ refocuses the ACC jets. In both the historic data and the altimeter data, the flow begins to move northeast, and the height contours converge, suggesting an intensified, higher velocity flow.

There is no evidence for the SAF turning north along the South American continental shelf and retroflecting when it meets the Brazil Current at $40^{\circ} \mathrm{S}$ [Peterson and Whitworth, 1989] (henceforth PW) because shallow water regions could not be analyzed, and the convoluted retroflecting SAF axis is not easily described by a double error function model. PW's estimate of the Falkland Current width, $150 \mathrm{~km}$ at 200 
m depth, is comparable to the full width of the Gaussian SAF $\left(4 a_{3}\right), 175 \mathrm{~km}$. They found the Falkland Current to be about $150 \mathrm{~km}$ wide at $200 \mathrm{~m}$ depth. A comparable measure of the width for the Gaussian jet model used in this analysis is 4 times the width parameter, $a_{3}$, for a total meridional width of $175 \mathrm{~km}$ at the surface.

\subsubsection{Southwest Atlantic, $55^{\circ}-0^{\circ} \mathrm{W}$}

Between about $55^{\circ}$ and $45^{\circ} \mathrm{W}$, the SAF and PF move northward through the Scotia Ridge. Because of the difficulties in capturing the retroflecting Falkland Current, the altimeter analysis shows the SAF slightly east of the front location identified by PW, but the PF follows a trajectory very similar to the one indicated by their analysis of hydrographic data.

Once the SAF and PF cross through the Scotia Ridge, they flow along the northern edge of the Ewing Bank and the Falkland Ridge. To the north of the Ewing Bank, altimeter height contours pinch together tightly, appearing to form a single intense jet near $45^{\circ} \mathrm{W}$. This is consistent with $\mathrm{PW}$, who found that the SAF and PF are very close together at this longitude and may sometimes merge to form a single jet. Along the Falkland Ridge to about $30^{\circ} \mathrm{W}$, the SAF and PF appear to be closely controlled by bathymetry in both altimetry and hydrography.

As the ACC flows eastward past the Islas Orcadas Rise, its path is less well defined and shows substantial meandering, which may mean that the flow is too weak and broad to be clearly identified by the automated nonlinear fitting code. The SAF and PF generally flow zonally across the Atlantic between $45^{\circ}$ and $50^{\circ} \mathrm{S}$. Hydrographic surveys of the central South Atlantic are limited, making verification of altimeter observations difficult, but dynamic topography maps [GMB78; Reid, 1989] indicate zonal flow. 


\subsubsection{Southeast Atlantic and the Southwest Indian Oceans, $0^{\circ}-40^{\circ} \mathrm{E}$}

The retroflecting Agulhas Current is the largest source of height variability in the southeastern Atlantic and southwestern Indian Oceans, but because it is not considered a circumpolar flow, it is not included in the reconstructed mean. The SAF and $\mathrm{PF}$ appear distinctly separate from the Agulhas, flowing eastward at $50^{\circ} \mathrm{S}, 10^{\circ}-15^{\circ}$ south of the retroflection region and the Crozet Plateau, consistent with hydrographic observations [Jacobs and Georgi, 1977; GMB78]. The SAF and PF are distinct features, though they converge at $33^{\circ} \mathrm{E}$, where a hydrographic survey by Read and Pollard [1993] also found them merged together.

\subsubsection{South Indian Ocean, $40^{\circ}-100^{\circ} \mathrm{E}$}

Between $40^{\circ}$ and $50^{\circ} \mathrm{E}$, the SAF and PF move abruptly northward in agreement with Jacobs and Georgi's [1977] STD sections. A deep passage in Crozet Plateau steers the SAF from its southern position at $40^{\circ} \mathrm{E}$ to the north side of the plateau, where Park et al. [1993] observed it. The PF moves northward east of Crozet Plateau. The SAF flows north of the Kerguelen-Gaussberg Plateau in both historic and altimeter data. The altimeter data indicate that the $\mathrm{PF}$ also takes a northward route around Kerguelen Island, but because the Gaussberg Plateau is too shallow for the tidal correction model, alternative paths to the south are not easily investigated. The hydrographic record does not offer a definitive map of the PF either; although the temperature indicator for the PF appears north of Kerguelen [Park et al., 1991], Park et al. [1993] hypothesize that the PF follows a southerly route but loops northward immediately to the east of Kerguelen to flow parallel to the SAF.

Thus the two strong fronts north of Kerguelen could be the SAF and STF, which have been observed in close proximity or even merged into a single front [Park et al., 1991]. Since altimeter data does not include thermohaline signatures and the 
height along each track is offset by a constant, this is difficult to verify. However, several contours which leave the ACC and enter the Indian Ocean, consistent with dynamic topography (Figure 2.9, GMB78, and Park et al., [1991]), suggest that the STF is separating from the SAF to recirculate through the Indian Ocean.

\subsubsection{Geographic Summary}

Overall, the SSH from the altimeter data agrees qualitatively with historic hydrographic measurements but supports the idea that narrow jets exist around the entire Southern Ocean. The techniques developed here for reconstructing mean flow in the Southern Ocean are specifically adapted to maintaining mesoscale spatial structure and were designed to be robust with respect to the types of errors in Geosat data. Nonetheless, the technique is applicable for newer altimeter data from ERS-1 or TOPEX-POSEIDON. Though TOPEX's $64^{\circ}$ turning latitude and coarser spatial resolution may pose difficulties for this type of investigation, the new satellite has better orbit tracking, and a more reliable large-scale geoid is now available, so that for basin scale analyses the mean SSH may be accessible, in addition to the variability. These improvements could permit the development of a better estimate of mean SSH by merging a mesoscale mean, derived using the techniques discussed in this chapter, with large-scale measured SSH.

\subsection{Summary}

The ACC has been modeled as two meandering Gaussian jets. Using the height as a guide to the jet locations and intensity, the mesoscale height field across the ACC jets was iteratively reconstructed using a nonlinear fitting procedure. The technique was applied to all 244 ascending and 244 descending satellite tracks which cover the Southern Ocean. Each ground track was repeated every 17 days, providing between 30 and 60 sets of measurements during the 30-month Geosat Exact Repeat Mission. 
Error estimates obtained using two types of Monte Carlo simulation indicated a negligible bias and $0.077 \mathrm{~m} \mathrm{RMS}$ error in the results. The resulting mean SSH was objective mapped with a meridional decorrelation distance of $100 \mathrm{~km}$ and a zonal decorrelation scale of $200 \mathrm{~km}$.

The reconstructed along-track mean ACC height is consistent with the presence of two narrow circumpolar jets, which have an average separation of $310 \mathrm{~km}$, although in a few locations they may merge to form a single jet. The jets meander about 1.7 times their width but less than the total average jet separation, so that the two jets are generally distinguishable in the mean field. The latitude of the ACC axes varies from $40^{\circ}$ to $60^{\circ} \mathrm{S}$, but the flow retains its sharp frontal structure all the way around its circumpolar trajectory. The substantial variations in latitude lead to variations in the baroclinic Rossby radius, and the widths of the SAF and PF adjust to match these changes in the Rossby radius. Estimates from hydrographic data suggest that the equivalent depth adjusts with latitude to make the transport proportional to the height difference across the jet. The flow is roughly steered by bathymetry. The jet model explains $40 \%$ to $70 \%$ of the altimeter height variance within the core of the ACC jets; in contrast to results from the Gulf Stream and the Kuroshio Extension, the percentage is smaller in regions of high variability, here associated with large topographic features. This points to the importance of eddy shedding and ring formation events in ACC dynamics.

\subsection{Appendix: Height Variance and Mean Veloc- ity}

High variance regions are closely linked with regions of large mean velocity; using Geosat data, Chelton et al. [1990] showed that the standard deviation of SSH in the Southern Ocean and the mean geostrophic velocity from hydrographic data have a geographical correlation of 0.64 . Despite the observational evidence, the connection 
between residual SSH and instantaneous SSH or mean surface velocity may not be intuitive. Here I develop a statistical framework to link the height variance to the mean jet velocity.

First, assume that the flow consists of a Gaussian jet which meanders about its mean position. Then the time-varying SSH may be written

$$
h(x, t)=A\left[\operatorname{erf}\left(\frac{x-x_{0}}{w_{1}}\right)+1\right]
$$

where $2 A$ represents the total height difference across the jet, $w_{1}$ is a measure of the half-width of the jet, and $x_{0}(t)$ is the center latitude.

Although the jet location errors discussed in section 2.3 are non-Gaussian, in the mean for all satellite tracks, the jet location distribution is Gaussian and spatially symmetric. Therefore assume the probability density function for $x_{\mathrm{o}}(t)$ is the distribution:

$$
P\left(x_{0}\right)=\frac{1}{w_{2} \sqrt{\pi}} \exp \left[-\left(\frac{x_{0}-\overline{x_{0}}}{w_{2}}\right)^{2}\right] \text {. }
$$

Thus a typical jet meanders about its mean position with a standard deviation $w_{2}$. Using the definition of the error function and combining (2.13) and (2.14), the mean SSH may be calculated as

$$
\begin{gathered}
\bar{h}(x)=A\left\{1+\frac{1}{w_{2} w_{1} \pi} \int_{-\infty}^{\infty} \int_{0}^{x} \exp \left[-\left(\frac{x^{\prime}-x_{0}}{w_{1}}\right)^{2}\right] d x^{\prime}\right\} \\
\left\{\exp \left[-\left(\frac{x_{0}-\overline{x_{0}}}{w_{2}}\right)^{2}\right] d x_{0}\right\} .
\end{gathered}
$$

By switching the order of integration, (2.15) is reduced to the simple expression for $\bar{h}$ :

$$
\bar{h}(x)=A\left[\operatorname{erf}\left(\frac{x-\overline{x_{0}}}{\sqrt{w_{1}^{2}+w_{2}^{2}}}\right)+1\right] .
$$

Correspondingly, the mean velocity is

$$
\bar{v}(x)=-\frac{g}{f} \frac{2 A}{\sqrt{\pi\left(w_{1}^{2}+w_{2}^{2}\right)}} \exp \left[-\left(\frac{x-\overline{x_{\circ}}}{\sqrt{w_{1}^{2}+w_{2}^{2}}}\right)^{2}\right] .
$$


The mean squared height may be written

$$
\begin{gathered}
\overline{h^{2}}=\frac{A^{2}}{w_{2} \sqrt{\pi}} \int_{-\infty}^{\infty}\left[1+\operatorname{erf}\left(\frac{x-x_{0}}{w_{1}}\right)\right]^{2} \\
\exp \left[-\left(\frac{x_{0}-\overline{x_{0}}}{w_{2}}\right)^{2}\right] d x_{\circ} .
\end{gathered}
$$

This expression is analytically difficult to work with because of the integrand containing erf ${ }^{2}$, but it may be computed numerically using plausible values for $w_{1}$ and $w_{2}$ to produce an estimate of the variance of $h$, that is $\overline{h^{2}}-\bar{h}^{2}$, which is plotted as a solid line in Figure 2.16. A jet's height variance is spatially similar to its squared mean velocity, shown as the long-dashed line in Figure 2.16. Both are basically Gaussian shapes centered at the mean axis of the current. Their widths differ by only a small margin. This suggests that by rescaling the amplitude of the height variance, we may obtain a reasonable proxy for the unknown mean velocity.

This result is a little clearer if we consider the limit where $w_{1}$ goes to zero, i.e., the jet becomes infinitesimally thin. In this case

$$
\bar{v}(x)=-\frac{g}{f} \frac{2 A}{\sqrt{\pi} w_{2}} \exp \left[-\left(\frac{x-\overline{x_{0}}}{w_{2}}\right)^{2}\right],
$$

and the variance is

$$
\overline{h^{2}(x)}-\bar{h}(x)^{2}=A^{2}\left[1-\operatorname{erf}^{2}\left(\frac{x-\overline{x_{0}}}{w_{2}}\right)\right] .
$$

While the expression for the variance is not identical to the squared velocity, both attain a maximum value at $\overline{x_{0}}$ and their widths do not differ substantially. ${ }^{4}$

\footnotetext{
${ }^{4}$ Although this appendix relies largely on kinematic arguments, the correspondence of the variance maximum and the center of the mean jet may also be explained through dynamical considerations. The zonal momentum equation states:

$$
\frac{\partial u}{\partial t}+\frac{\partial(u u)}{\partial x}+\frac{\partial(v u)}{\partial y}+f v=-\frac{1}{\rho_{0}} \frac{\partial p}{\partial x}+\frac{\partial}{\partial z} \frac{\tau^{x}}{\rho_{0}}+A_{m} \nabla^{4} u .
$$

Averaged over sufficiently long time intervals the tendency term is negligible, and the time-averaged zonal momentum equation may be written:$$
\frac{\partial \overline{u^{\prime} u^{\prime}}}{\partial x}+\frac{\partial \overline{v^{\prime} u^{\prime}}}{\partial y}+\frac{\partial \overline{u v}}{\partial x}+\frac{\partial \overline{v u}}{\partial y}+f \bar{v}=-\frac{1}{\rho_{0}} \frac{\partial \bar{p}}{\partial x}+\frac{\partial}{\partial z} \frac{\overline{\tau^{x}}}{\rho_{0}}+A_{m} \nabla^{4} \bar{u} .
$$ 


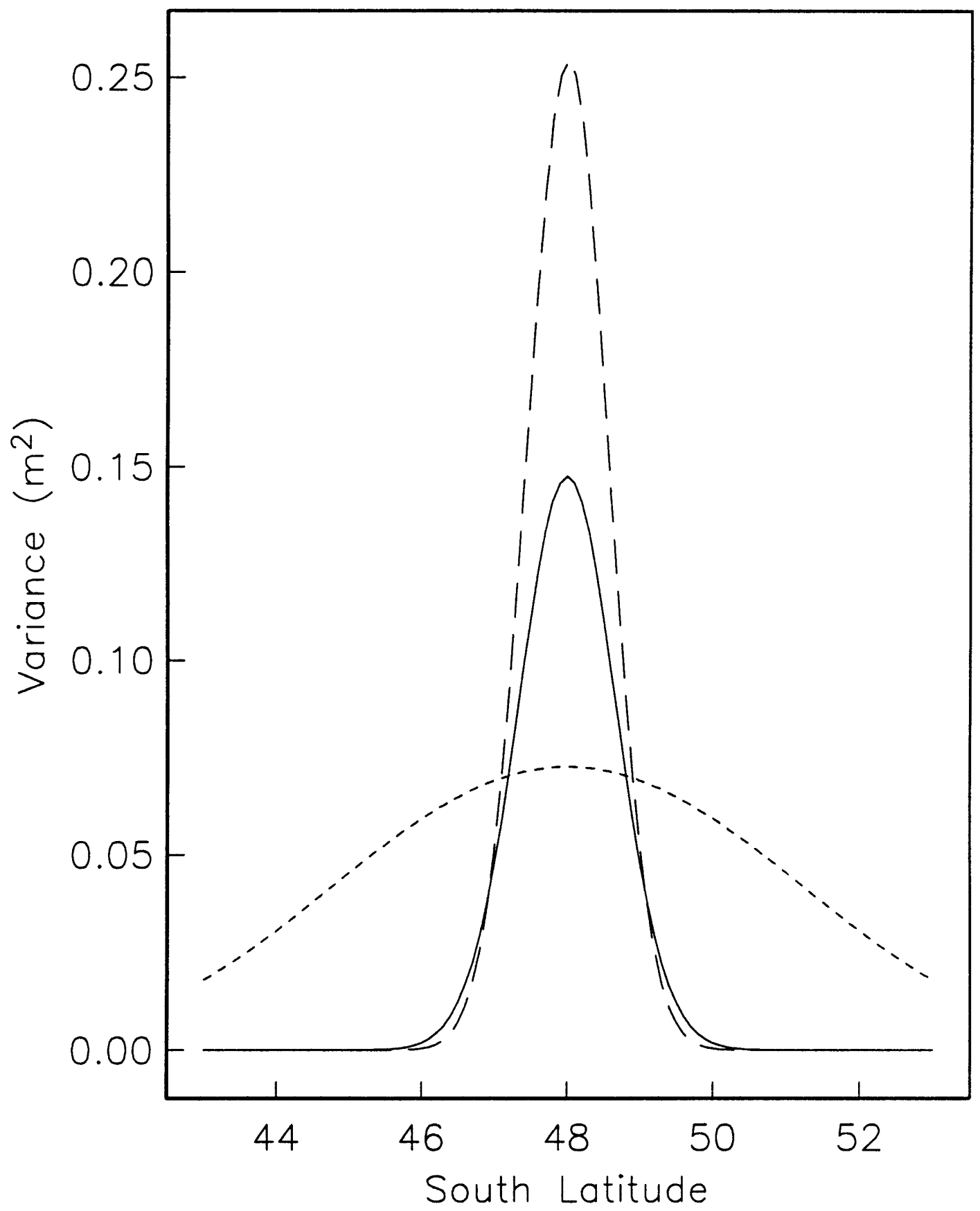

Figure 2.16: Predicted variance of altimeter height measurements. The solid line represents the SSH variance corresponding to a meandering Gaussian velocity jet, with parameters $w_{1}=0.5$ and $w_{2}=1.0$. The short-dashed line represents the variance of a ring modeled as a Gaussian height bump with parameters $w_{1}=0.5$ and $w_{2}=4.0$. For comparison, the long-dashed line shows the mean velocity squared $\left(\bar{v}^{2}\right)$ for the meandering jet. By rescaling the measured height variance, we can obtain a plausible first approximation for $\bar{v}^{2}$. 
Rings also have time-varying mesoscale features and may be mistaken for jets. However, if we assume that a typical ring moves substantially further than a jet, then its variance is likely to be smaller than the jet variance. To see this, parameterize the ring as the sum of an error function and a complimentary error function separated

Projected into stream coordinates, the equation remains essentially identical, except that $x$ and $y$ represent the along-stream and cross-stream directions, and $u$ and $v$ the along-stream and crossstream velocities. The mean cross-stream geostrophic velocity, $\frac{1}{\rho_{0}} \frac{\partial \bar{p}}{\partial x}$ is by definition zero. Thus, (neglecting curvature terms) at the ocean surface, in stream coordinates:

$$
\frac{\partial \overline{u^{\prime} u^{\prime}}}{\partial x}+\frac{\partial \overline{v^{\prime} u^{\prime}}}{\partial y}+\frac{\partial \overline{u u}}{\partial x}+f \bar{v}_{a g}=\frac{\partial}{\partial z} \frac{\overline{\tau^{x}}}{\rho_{o}}+A_{m} \nabla^{4} \bar{u}
$$

Assuming that eddy features vary more slowly along streamlines than across streamlines, the along stream terms, $\frac{\partial \overline{u^{\prime} u^{\prime}}}{\partial x}+\frac{\partial \overline{u u}}{\partial x}$ are small, and the diffusion term is dominated by its cross-stream component, $\frac{\partial^{4} \bar{u}}{\partial y^{4}}$. Estimates from altimeter data [Morrow et al., 1994] and from numerical model output [McWilliams it et al., 1978; Wolff et al., 1991; Treguier and McWilliams, 1990] have suggested that transient eddies drive the mean flow. This means that the gradient of $u^{\prime} v^{\prime}$ should change sign along the axes of the ACC jets. Thus we expect:

$$
0 \approx-\frac{\partial \overline{v^{\prime} u^{\prime}}}{\partial y}
$$

Using quasi-geostrophic scaling arguments, we find:

$$
\begin{aligned}
0 & \approx+\frac{\partial}{\partial y}\left(\frac{1}{f^{2}} \frac{\partial p^{\prime}}{\partial x} \frac{\partial p^{\prime}}{\partial y}\right) \\
& \approx-\frac{\partial}{\partial y}\left[\frac{1}{f^{2} \Delta x \Delta y}\left(\overline{p_{i, j}^{\prime} p_{i, j}^{\prime}}+\overline{p_{i+1, j}^{\prime} p_{i, j}^{\prime}}+\overline{p_{i, j}^{\prime} p_{i, j+1}^{\prime}}+\overline{p_{i+1, j}^{\prime} p_{i, j+1}^{\prime}}\right)\right]
\end{aligned}
$$

where $i$ and $j$ are the represents discrete grid points in the zonal and meridional directions, and $\Delta x$ and $\Delta y$ are the separations between adjacent points. If pressure variations at adjacent points are uncorrelated, then:

$$
\begin{aligned}
0 & \approx-\frac{\partial}{\partial y}\left(\frac{1}{f^{2} \Delta x \Delta y} \overline{p^{\prime} p^{\prime}}\right) \\
& =-\frac{\partial}{\partial y}\left(\frac{g^{2}}{f^{2} \Delta x \Delta y} \overline{\eta^{\prime} \eta^{\prime}}\right) .
\end{aligned}
$$

Thus the center of the jet should occur at a cross-stream extrema of the height variance $\overline{\eta^{\prime} \eta^{\prime}}$. The height variance maximum identifies the jet axis. Morrow et al. [1994] use the equivalent of (2.26) expressed in terms of the velocity variance to identify the ACC axis with heavily smoothed data, but for the analysis in this chapter, the along track data are relatively noisy and the height variance is an easier quantity to interpret. 
by the ring diameter $2 d$,

$$
h_{\text {ring }}(x)=A \operatorname{erf}\left(\frac{x-x_{0}-d}{w_{1}}\right)-A \operatorname{erf}\left(\frac{x-x_{\circ}+d}{w_{1}}\right) .
$$

Using the same probability distribution as above, the mean SSH is given by

$$
\bar{h}(x)=A \operatorname{erf}\left(\frac{x-x_{\circ}-d}{\sqrt{w_{1}^{2}+w_{2}^{2}}}\right)-A \operatorname{erf}\left(\frac{x-x_{\circ}+d}{\sqrt{w_{1}^{2}+w_{2}^{2}}}\right) .
$$

Again, the expression for the variance is best calculated numerically. The short dashed line in Figure 2.16 indicates that a typical widely meandering ring will have a broad flat variance with a smaller amplitude than the narrow jet variances. The shape and amplitude of bumps in the height variance provide a means to distinguish jet and eddy variance features. If the ring disappears some of the time, then its variance is correspondingly reduced.

The height variance is by no means a foolproof measure of the squared mean velocity. Local asymmetries in the jet distributions may alter the mean RMS height from the simple model description, so that the location of maximum variance does not coincide with the mean jet axis. Nonetheless, this approach provides a sensible way to use the available information as a first guess to feed into our error function model.

How well does this approximation work? Figure 2.17 shows an example of the height variance along one track compared to the mean velocity estimated using the Gaussian model. The synthetic velocity (dashed line) shows the SAF near $49.5^{\circ} \mathrm{S}$ and the PF near $53^{\circ} \mathrm{S}$. The solid line represents the RMS SSH along the same ground track. With the selected scaling factor, the velocity of the SAF front is underestimated by about $25 \%$ and the velocity of the PF is overestimated by about $10 \%$, which is sufficient for a first-order guess. The jet axes determined from the RMS height are located within $0.3^{\circ}$ latitude of the jet maximum velocities. 


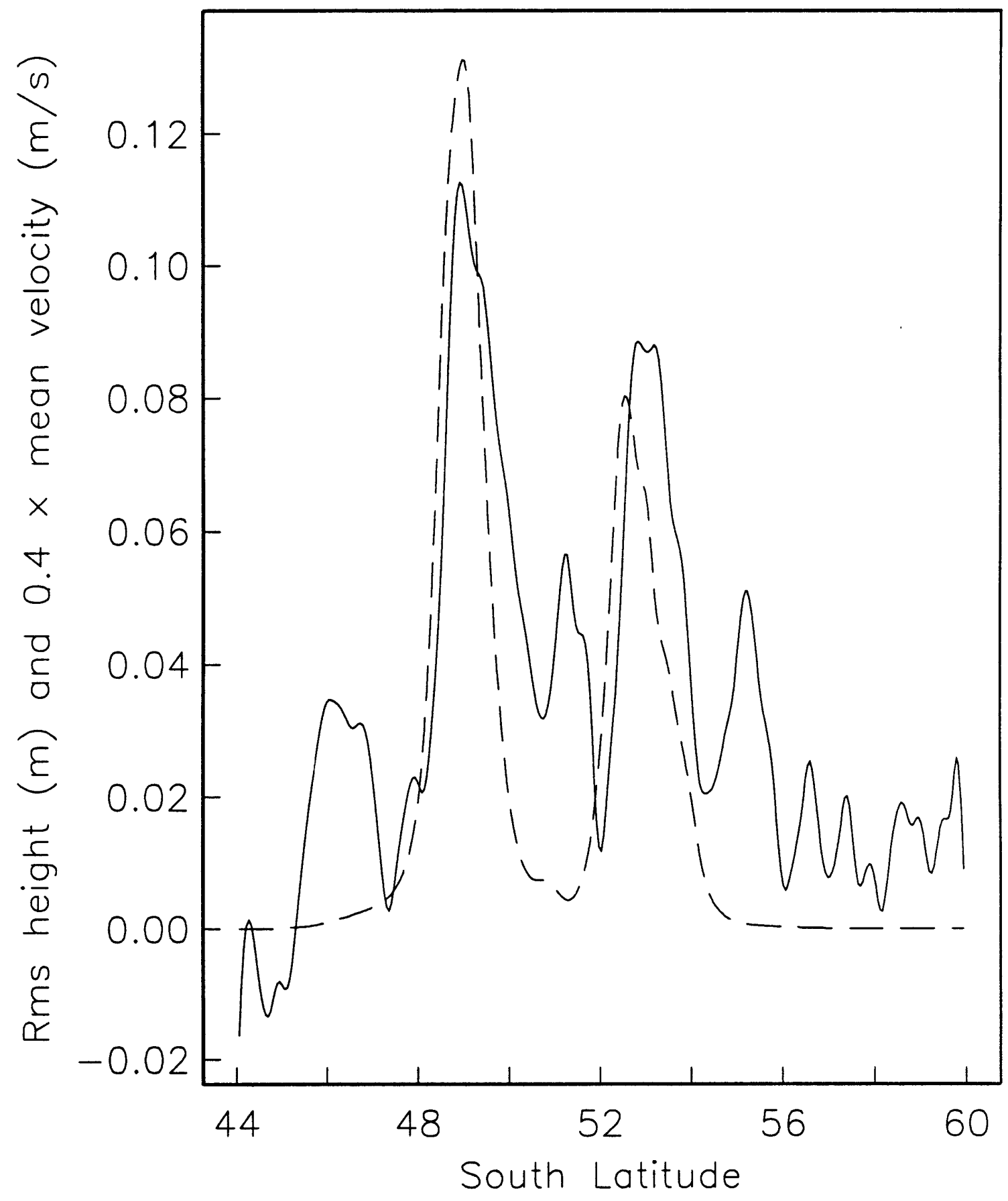

Figure 2.17: A comparison of the height standard deviation along track d001 (solid line) and estimated mean velocity from the Gaussian jet model (dashed line). 


\section{Chapter 3}

\section{Variability}

\subsection{Introduction}

Altimeter measurements clearly illustrate the intensity of eddy variability throughout the Southern Ocean and particularly along the axis of the ACC [Shum et al., 1990; Chelton et al., 1990; chapter 2]. Theoretical work and numerical models have indicated that this high eddy variability is critical to the overall dynamical balances governing the ACC; transient eddies are most commonly invoked to transfer momentum input by surface wind stress downward to depths where topographic form drag can dissipate it.

Observational studies have characterized the ACC as having short spatial and temporal scales consistent with the presence of substantial eddy activity. Using historic hydrographic data, Lutjeharms and Baker [1980] analyzed the spatial structure of the Southern Ocean; their results indicated rapid spatial decorrelation, with length scales less than $150 \mathrm{~km}$. From satellite-tracked surface drifting buoys, Patterson [1985] estimated that the majority of kinetic energy in the Southern Ocean has a time scale of less than one month. Current meter results, summarized by Nowlin and Klinck 
[1986], have indicated that most of the energy is associated with time scales of 20-50 days in the upper ocean and 7-20 days at depth [Inoue, 1985]. Spatial decorrelation length scales for temperature estimated from mooring data were $80-100 \mathrm{~km}$ [Sciremammano et al., 1980].

In spite of the short spatial scales of eddy variability, in studies of transport fluctuations, investigators have usually conjectured that the ACC varies uniformly along its entire circumpolar path. This assumption is made for two reasons: First existing wind information from ship measurements and from the European Centre for Medium-Range Weather Forecasts' (ECMWF) model [van Loon, 1972; Large and van Loon, 1989], are dominated by global-scale, semi-annual fluctuations. If the ACC is driven by these relatively uniform changes in the wind, then its transport might be predicted to vary similarly. Second, regardless of the forcing, the continuous and zonally connected character of the ACC means that transport variations in any given location should lead to transport variations everywhere.

The presumption of large-scale coherent fluctuations in transport appears to contradict observations which have emphasized the small spatial scales and large eddy variability of the Southern Ocean. Nonetheless, because of the predicted large-scale coherence, most of the transport monitoring of the ACC has been done in Drake Passage, where the flow is confined to a narrow latitude range. Measurements in Drake Passage are assumed to apply to the entire system. Wearn and Baker [1980] showed that transport variations estimated from pressure gauges in Drake Passage were significantly correlated with fluctuations in the zonally averaged winds, with a 9 day lag. However the reliability of their results has remained uncertain. Chelton [1982] argued that the high correlations Wearn and Baker observed could be an artifact of comparing two signals with strong seasonal components. Extended pressure gauge records have indicated substantial interannual variability in ACC transport both at Drake Passage [Whitworth and Peterson, 1985] and between Amsterdam and Kerguelen Islands [Vassie et al., 1994]; thus interpretation of annual and semi-annual 
signals is potentially misleading because of the relatively small amplitudes of the harmonics relative to longer period variations. Using surface drifters throughout the entire Southern Ocean from a one year interval, Large and van Loon [1989] found a substantial annual and semi-annual velocity signal, accounting for $30 \%$ to $85 \%$ of the oceanic variability depending on the location, but they saw substantial zonal variation and little spatial correlation between low-frequency wind and drifter measurements, in contradiction with the premises of Wearn and Baker [1979]. As the ambiguities in observational results indicate, in situ measurements have not provided sufficient information to reconcile our understanding of large-scale forcing in the Southern Ocean with the observed mesoscale eddy variability.

The global synoptic coverage provided by satellite altimeter offers a means to address variability processes more carefully. Chelton et al. [1990] used Geosat altimeter measurements at groundtrack cross-over points to examine Southern Ocean variability. Their results provided little evidence for circumpolar scale variability and instead suggested variability might occur on basin-wide or regional scales. However, their results may not be representative of ACC transport changes: results in chapter 2 indicated that transient eddies not associated with jet meandering accounted for a higher percentage of the variance in the ACC than in the Gulf Stream or the Kuroshio, so that large-scale analyses might be dominated by eddy variability and might not detect coherent transport fluctuations.

This study will use mesoscale analysis techniques to more specifically examine scales of variability in the Southern Ocean, taking advantage of the collinear processed data and reconstructed mean sea surface height developed in chapter 2 . In section 3.2, length and time scales of variability are estimated from data and used to map instantaneous sea surface height. The resulting figures illustrate how the substantial variability appears to be associated with local mesoscale processes such as meandering and ring formation along the ACC fronts, rather than large-scale fluctuations. In section 3.3, temporal and spatial scales of surface transport variability 
will be estimated by computing empirical orthogonal functions. One might envision a scenario in which a constant background level of eddies controlled the momentum balance, while ACC transport responded directly and in a globally coherent way to wind forcing. However, the results of this study, summarized in section 3.5, will instead suggest that mesoscale eddy motions dominate Southern Ocean surface transport variability, and there is little evidence for coherent transport fluctuations.

\subsection{Scales of Variability}

\subsubsection{Time Varying Sea Surface Height}

Length-scales of mesoscale variability may be defined in a number of ways. For example, Stammer and Böning [1992], in their investigation of Atlantic Ocean variability scales computed along-track wavenumber spectra and integral length-scales of autocovariance functions. For this study, in order to objectively map sea surface height variability information, a Gaussian decorrelation function will be fit to autocovariances computed from the data. The autocovariance provides a measure of the cross-correlation of variability at points separated in time and space. If a large region fluctuates in unison, then the autocovariance will be high and will decrease slowly as a function of distance. If spatial fluctuations occur over short distances, then measurements taken at nearby points will be uncorrelated, so that the autocovariance will decrease rapidly with distance. Similarly, the time scales over which the autocovariance decreases indicate the temporal scales of variability of the measured data.

Sea surface height data were employed from 244 ascending and 244 descending collinear groundtracks from the Geosat altimeter. The raw data were initially processed to remove outliers, corrected, and filtered following the procedure outlined in chapter 2. Along-track spatial and temporal autocovariances were computed for 


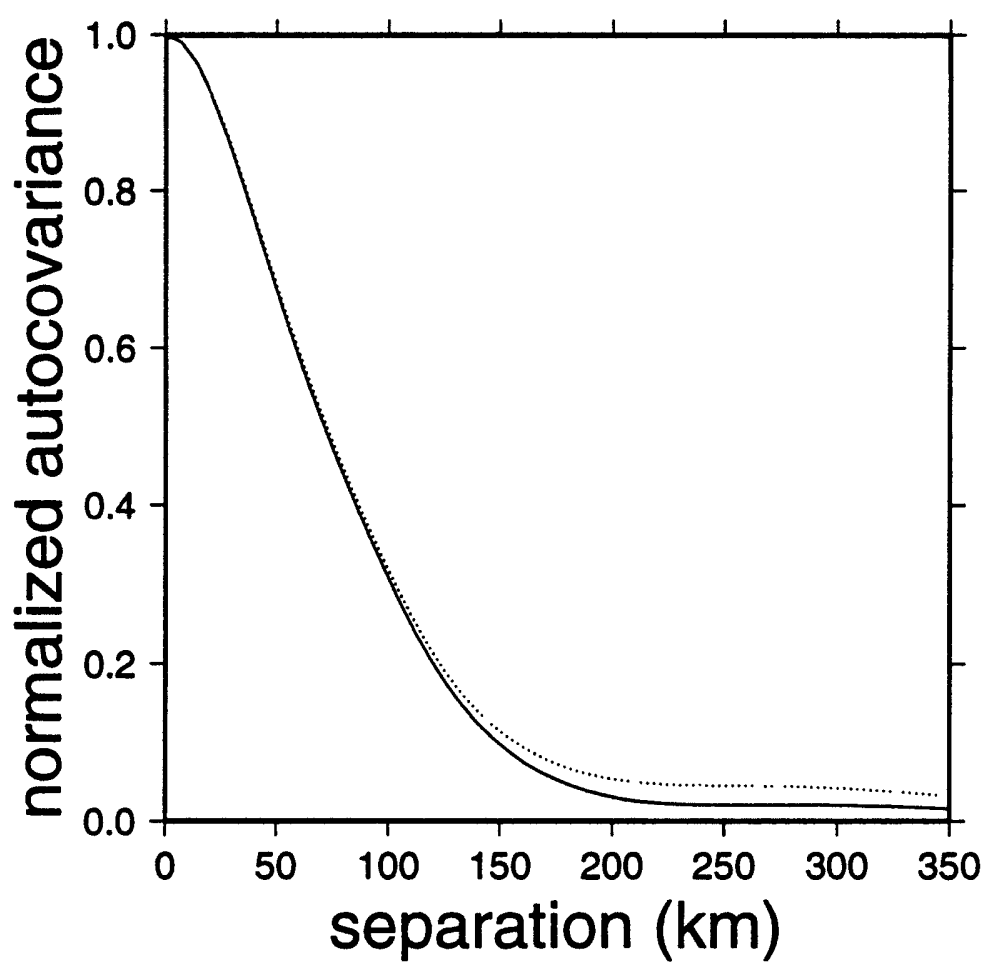

Figure 3.1: Spatial autocovariance for ascending (solid) and descending (dotted) tracks, averaged over all ground tracks and normalized to a maximum amplitude of 1 at 0 lag. Differences between ascending and descending tracks are not statistically significant.

a $350 \mathrm{~km}$ swath centered around the core velocity jets of the ACC. Functional parameters were then fit to the autocovariance data to define length and time scales of variability.

Stammer and Böning [1992] found that length scales in the ocean varied considerably with latitude, but were relatively constant as a function of longitude; careful examination of the autocovariances in this analysis supports their findings and also suggests that the temporal autocovariance does not vary regionally. Therefore, since the latitude of the ACC changes by only about $20^{\circ}$, no geographic variation is included in the decorrelation functions defined for this study. Figure 3.1 shows the normalized mean spatial autocovariance for ascending and descending tracks and Figure 3.2 shows the normalized mean temporal autocovariance. The negative temporal auto- 


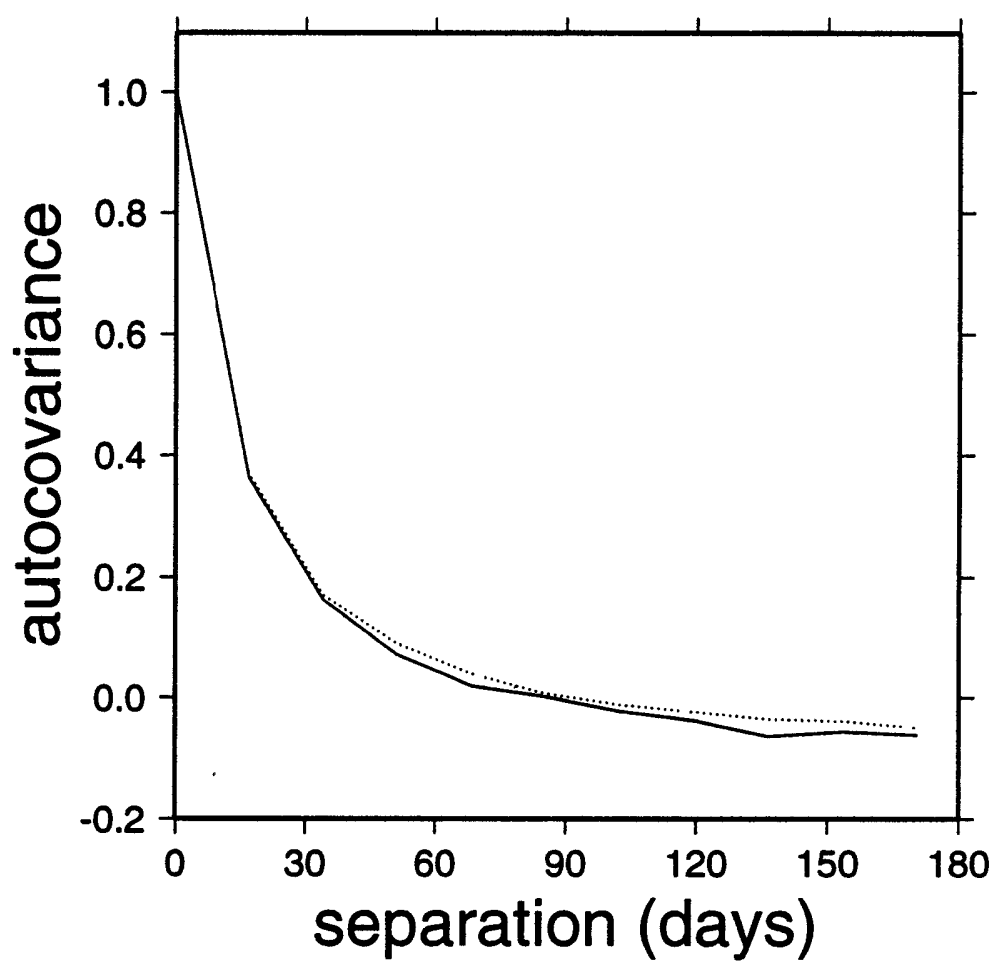

Figure 3.2: Temporal autocovariance for ascending (solid) and descending (dotted) tracks, averaged over all ground tracks and normalized to a maximum amplitude of 1 . Differences between ascending and descending tracks are not statistically significant. 
covariance at lags greater than 80 days is an artifact of the finite duration of the time series. To understand this, think of each measurement as:

$$
s_{i}=s_{i}^{\prime}+\bar{s}+\tilde{s}
$$

where $s_{i}$ is the instantaneous measurement, $s_{i}^{\prime}$ is the deviation from the sample mean, $\bar{s}+\tilde{s}$ is the mean of all sampled $s_{i}$, and $\bar{s}$ is the mean which would be found if the ensemble of $s_{i}$ contained an infinite number of elements. The autocovariance is computed by removing the mean from $s_{i}$. Therefore, the computed autocovariance is:

$$
\tilde{c}(t)=\frac{1}{N-t} \sum_{i=1}^{N-t} s_{i} s_{i+t}
$$

while the correct autocovariance for an infinitely long data set is:

$$
\hat{c}(t)=\frac{1}{N-t} \sum_{i=1}^{N-t} s_{i} s_{i+t}+\tilde{s} \tilde{s} .
$$

The computed autocovariance $\tilde{c}$ therefore differs from the correct autocovariance $\hat{c}$ by a constant $\tilde{s} \tilde{s}$, which is positive. Thus the unknown $\tilde{\boldsymbol{s}} \tilde{s}$ must be added to $\tilde{c}$ in order to determine the real autocovariance; for this study the value of $\tilde{s} \tilde{s}$ is estimated by assuming that the autocovariance should drop to zero at large time separations but should not be negative.

Based on the autocovariances represented in Figures 3.1 and 3.2, the correlation function is assumed to be Gaussian in space and exponential in time:

$$
c_{i, j}=D \exp \left[-\frac{\Delta d_{i j}^{2}}{B^{2}}\right] \exp \left[-\left|\frac{\Delta t_{i j}}{T}\right|\right]+C \delta_{i j} .
$$

By least-squares fitting (3.4) to the covariances determined from the data, the amplitude $D$ is estimated to be $0.012 \mathrm{~m}^{2}$, the length-scale, $B$, to be $85 \mathrm{~km}$, and the time-scale, $T$, to be 34 days. For measurements from the same satellite pass along a single ground track the zero lag offset, $C$, is $0.001 \mathrm{~m}^{2}$, corresponding to the $3 \mathrm{~cm}$ rms noise assumed for the Geosat altimeter. When different ground tracks or different satellite passes are compared, $C$ is $0.004 \mathrm{~m}^{2}$, corresponding to $6.5 \mathrm{~cm}$ rms for instrument noise, orbit error, and residual atmospheric and EM bias corrections. 
The 34-day time scale is comparable to upper ocean time scales of 20 to 50 days estimated from Drake Passage current meter data [Inoue, 1985] and to e-folding scales of 20 to 30 days estimated from Southern Ocean temporal autocorrelation functions plotted by Minster and Gennero [1995] in their analysis of ERS-1 altimeter measurements on a 3-day repeat. The $85-\mathrm{km}$ length scale is approximately the same as the diameters of smaller observed rings, which have varied in size from 60 to 200 $\mathrm{km}$ [Bryden, 1983] and is comparable to the spatial decorrelation length scales for temperature found by Sciremammano et al. [1980].

The mean sea surface height, objectively mapped in chapter 2 , varied more rapidly in the meridional direction than in the zonal direction. However, within measurement constraints, no equivalent anisotropy is identifiable in sea surface height variations. Length scales of eddy variability are approximately the same along ascending and descending tracks. Since the spatial and temporal scales of eddies are comparable to the track separation in the latitudes of the ACC, adjacent tracks cannot be combined to examine zonal wavenumber spectra in greater detail. In situ measurements in Drake Passage are consistent with the altimeter analyses: although velocity variations are anisotropic, temperature fluctuations, which are a better analog to sea surface height, are isotropic [Sciremammano et al., 1980].

The correlation function (3.4) is used to objectively map along-track sea surface height anomalies using the technique outlined by Bretherton et al. [1976]. Objective mapping uses irregularly sampled data points along with their errors and decorrelation functions to determine the best estimate of the physical quantity on a regular grid. The mean sea surface height is added to the mapped sea surface height anomaly field. Figure 3.3 shows the resulting instantaneous sea surface height for December $28,1986$.

Objective mapping has the advantage of readily providing error estimates derived from the data covariance functions and error estimates. For the instantaneous case, errors in sea surface height are between 5 and $7 \mathrm{~cm}$ over most of the domain. 


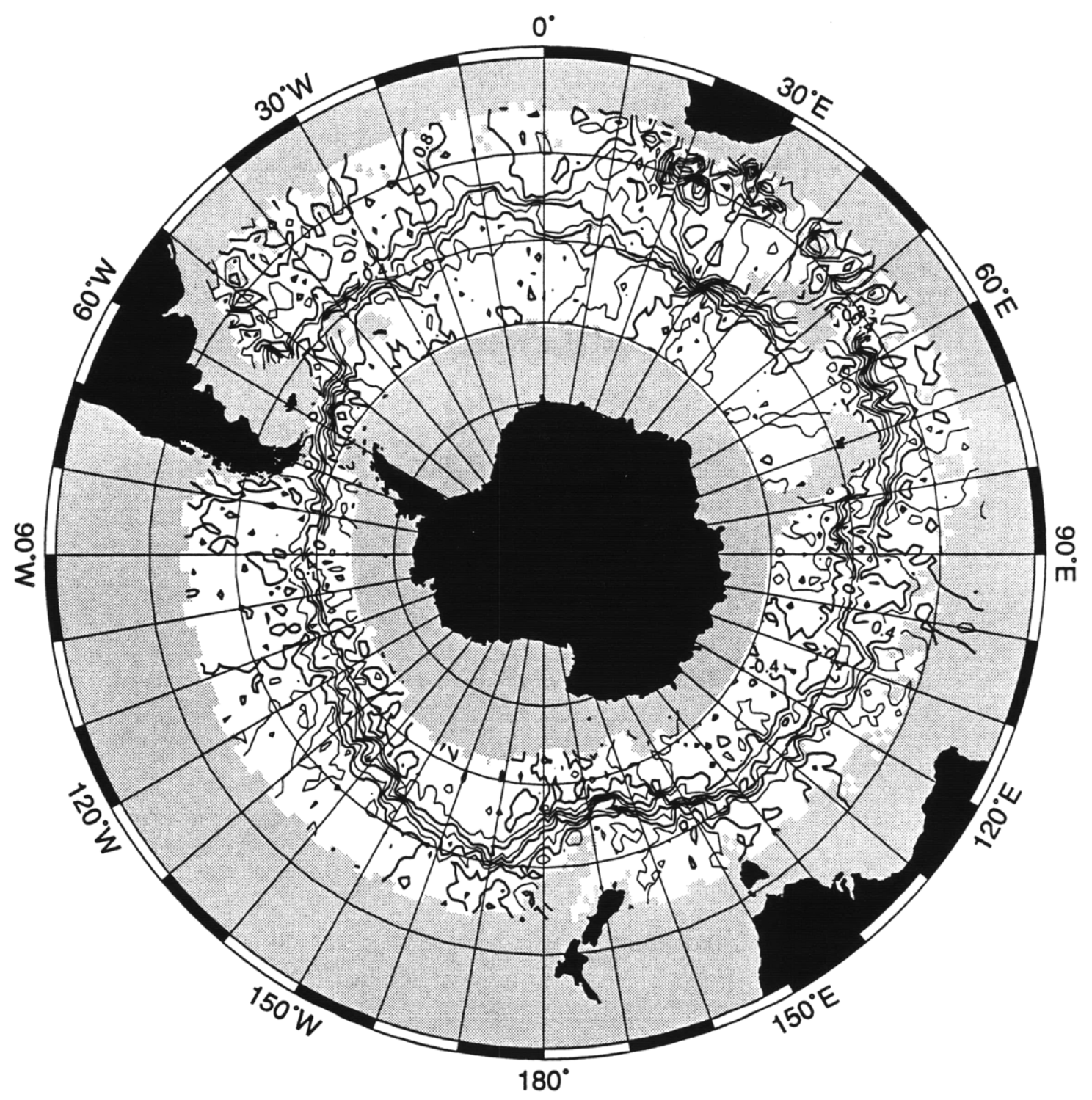

Figure 3.3: Synoptic map of reconstructed sea surface height for 28 December 1986, with a contour interval of $0.1 \mathrm{~m}$. Regions with no data or height errors greater than $7 \mathrm{~cm}$ are gray, and contours are not shown when the error exceeds $12 \mathrm{~cm}$. Over most of the region errors are between 5 and $7 \mathrm{~cm}$. 
Error estimates for derived quantities such as geostrophic velocities are more subtle. Since they will be necessary for the analysis in chapter 4 , they are discussed in the appendix (section 3.6).

\subsubsection{Aliasing and Altimeter Sampling}

The sampling pattern used by the altimeter has led to considerable concern about aliasing problems which might affect the data interpretation. Wunsch [1989] used a singular value decomposition to estimate error covariances of Fourier components for one 17-day period and found that uncertainties in the amplitudes could exceed $50 \%$, but he did not consider time periods longer than 17 days or spatial scales shorter than $6^{\circ}$, which might be more relevant for the mesoscale methods used in this study. Individually, each of the satellite tracks should not be subject to severe aliasing problems (aside from energy at 17-day intervals aliasing into the mean) since the basic analysis in chapter 2 treats each measurement as a single realization rather than as a time series. However, when adjacent tracks are mapped together or when results are analyzed as a time series, aliasing issues become a serious consideration.

The decorrelation time scale of 34 days estimated from the data is at the lower limit imposed by the Nyquist frequency for the Geosat altimeter's 17-day sampling interval but suggests that the dominant scales of temporal variability should be captured by Geosat. Since current meter observations in the Southern Ocean have indicated high vertical coherence for low-frequency fluctuations [Pillsbury et al., 1979; Whitworth and Peterson, 1985; Whitworth et al., 1991], geostrophic surface velocities are predicted to have spectra similar to those computed from current meter measurements. Energy spectra computed from current meters in Drake Passage and southeast of New Zealand, located between $3000 \mathrm{~m}$ and $300 \mathrm{~m}$ depth are red between the lowest frequency adequately sampled ( 1 cycle per 50 days) and just below the diurnal tidal frequency ( 1 cycle per 2 days) [Pillsbury et al., 1980; Bottero et al., 1981; Pillsbury et al., 1981]. For current meters above $1000 \mathrm{~m}$ depth, the diurnal 
and semi-diurnal tidal peaks are one to two orders of magnitude less energetic than the energy at 1 cycle per 34 days. Figure 3.4 shows the spectral density of pressure measured from a mooring on the north side of Drake Passage. The pressure spectrum is essentially red, even at low frequencies. A substantial energy peak at 2 cycles per day corresponds to the M2 tidal peak. Pressure is most directly compared with sea surface height variability measured by the altimeter, shown in Figure 3.5, which indicates a red spectrum for the sampled frequency range, roughly between 0.001 cycles per day and 0.03 cycles per day. Since Figure 3.4 indicates that the tidal peak is less energetic than the background spectral density at frequencies sampled by the altimeter, and much of the tidal energy is removed from the altimeter measurements in the data processing, these measurements indicate that significant amounts of energy are unlikely to be aliased by the Geosat altimeter's 17-day sampling interval. Minster and Gennero [1995] performed a detailed analysis using ERS-1 altimeter data on a 3 -day repeat orbit; their results indicated that only 10 or $15 \%$ of the sea surface height variance is not resolved by Geosat. The current meter and ERS-1 results are consistent with Jacobs et al.'s [1992] analysis of M2 tidal aliases in the Geosat annual signal: taking advantage of the spatial structure of the tidal alias, they performed a least-squares fit to estimate its phase and amplitude, which varied between 1 and 5 $\mathrm{cm}$ in the Southern Ocean. Errors of this magnitude fall well within the error bars of the time dependent signal, but the example of the M2 tide is indicative of the types of errors which might affect the data interpretation.

The decorrelation length scale of $85 \mathrm{~km}$ is substantially longer than the 7 $\mathrm{km}$ along-track sampling interval but very close to the between track spacing which varies with latitude from 75 to $125 \mathrm{~km}$. Thus, while the along-track spacing of 7 $\mathrm{km}$ is more than sufficient to sample the observed horizontal variability, the spatiotemporal aliasing in large regions poses more difficult problems which are not easily addressed through a simple analysis of historic data sets. More thorough analysis using the strategies outlined by Wunsch [1989] and taking advantage of the sampling 
UNFIITERED PRESSURE. 525 METERS AT NS-1000.

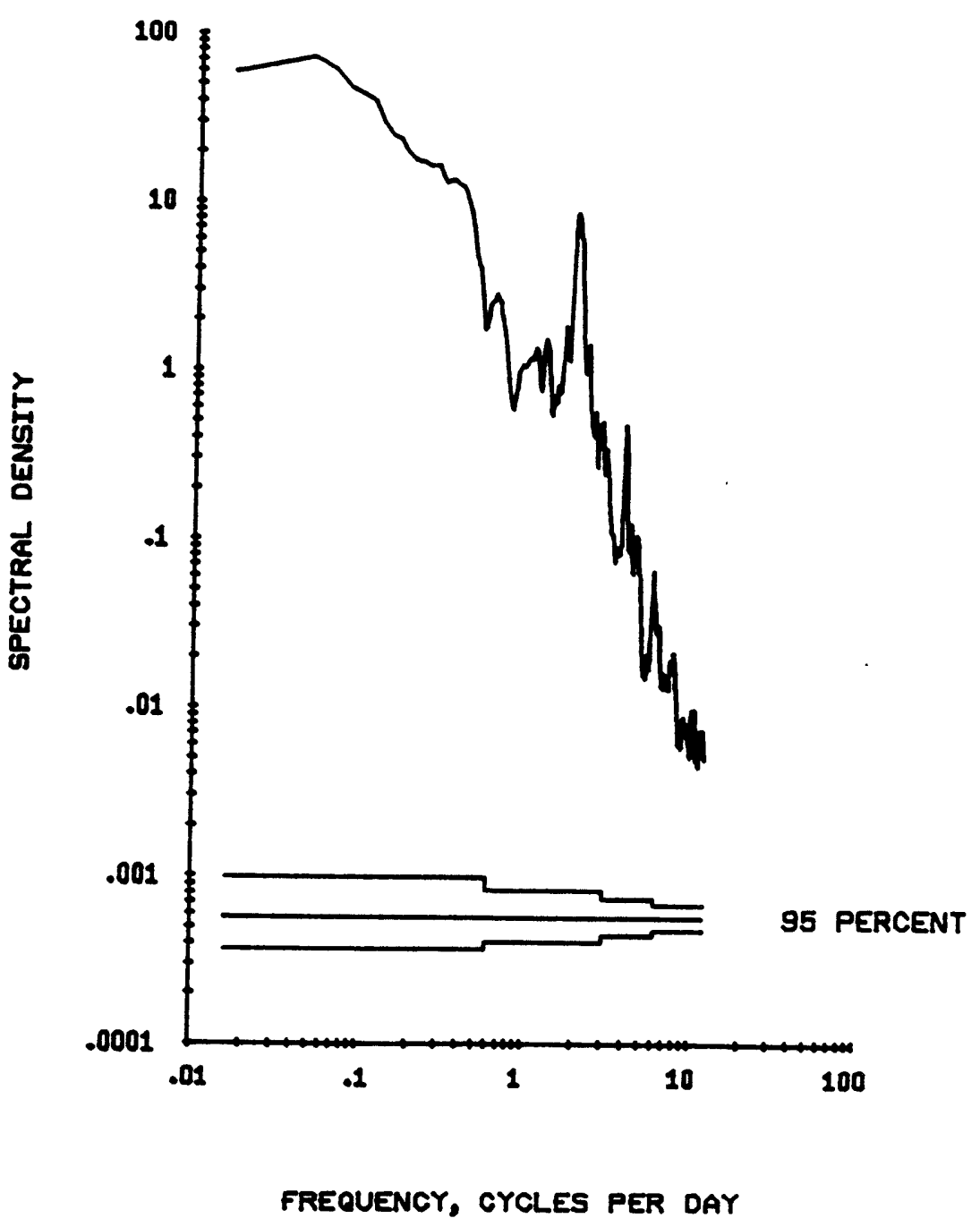

Figure 3.4: Spectral density for pressure measurements in 525 meters of water on the north slope of Drake Passage ( $56^{\circ} 34.3^{\prime} \mathrm{S} ; 6^{\circ} 6^{\circ} 55.5^{\prime} \mathrm{W}$ ) from the Drake 79 Experiment from 14 January 1979 to 29 January 1980, reproduced from Bottero et al. [1981]. 


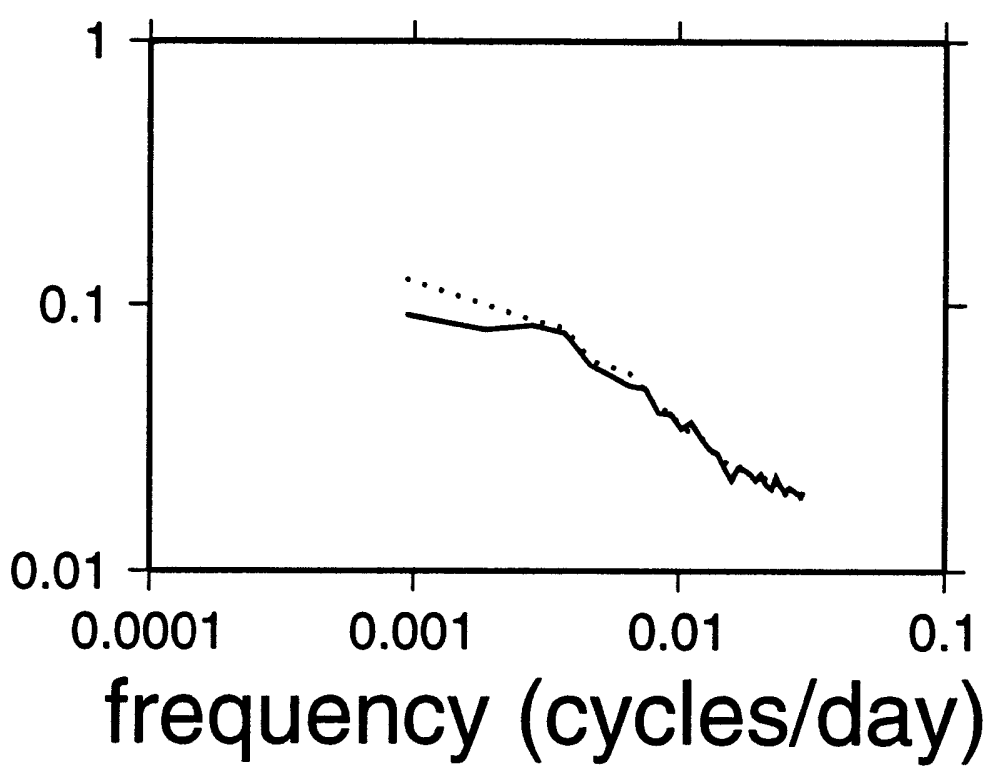

Figure 3.5: Mean spectral density for sea surface height measurements (in $\mathrm{cm}^{2} \mathrm{~s}^{-2}$ ) averaged for all 244 ascending (solid line) and 244 descending (dotted line) tracks in a $700 \mathrm{~km}$ wide swath centered on the mean axis of the ACC. Spectra are computed using the method for irregularly spaced data described by Ferraz-Mello [1981]. Statistical errors are small as each line represents an average of over 20,000 spectra.

patterns from newer altimeters should ultimately help to identify likely ranges of aliased energy.

\subsubsection{Local Eddy Processes}

Now we will more carefully examine specific mesoscale processes in a sequence of sea surface height maps for the region of intense eddy activity south of New Zealand, keeping in mind that the Nyquist frequency and wavenumber for the Geosat altimeter marginally resolve the spatial and temporal scales over which Southern Ocean sea surface height decorrelates so that the mapped fields may alias some features. Figure 3.6 indicates the mean sea surface height for the region reconstructed following the procedure outlined in chapter 2 . Shown superimposed over bathymetry from the ETOPO5 (5 minute resolution bathymetric data) the mean sea surface height is closely steered by bathymetry along the Southeast Indian Ridge, through the Mac- 


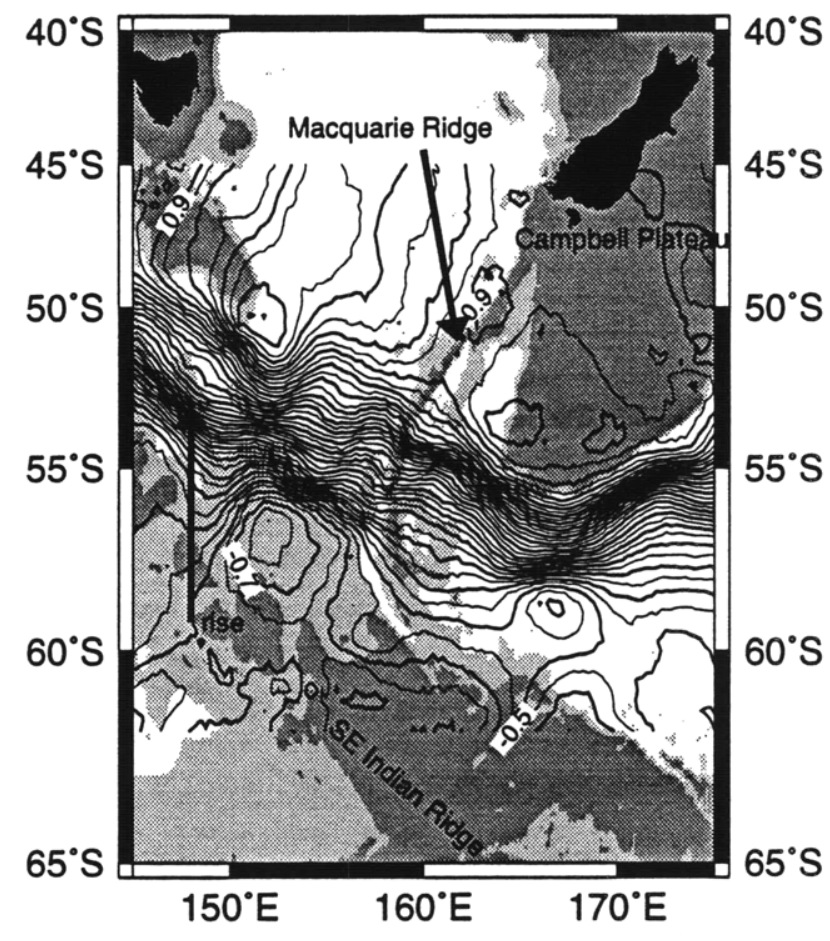

Figure 3.6: Mean sea surface height south of Australia and New Zealand, reconstructed using the technique described in chapter 2. Sea surface height is in meters with a $0.1 \mathrm{~m}$ contour interval. Results are superimposed on ETOPO5 5 minute resolution bathymetric data. Dark gray regions are shallower than $3000 \mathrm{~m}$, and lighter gray regions shallower than $4000 \mathrm{~m}$. 


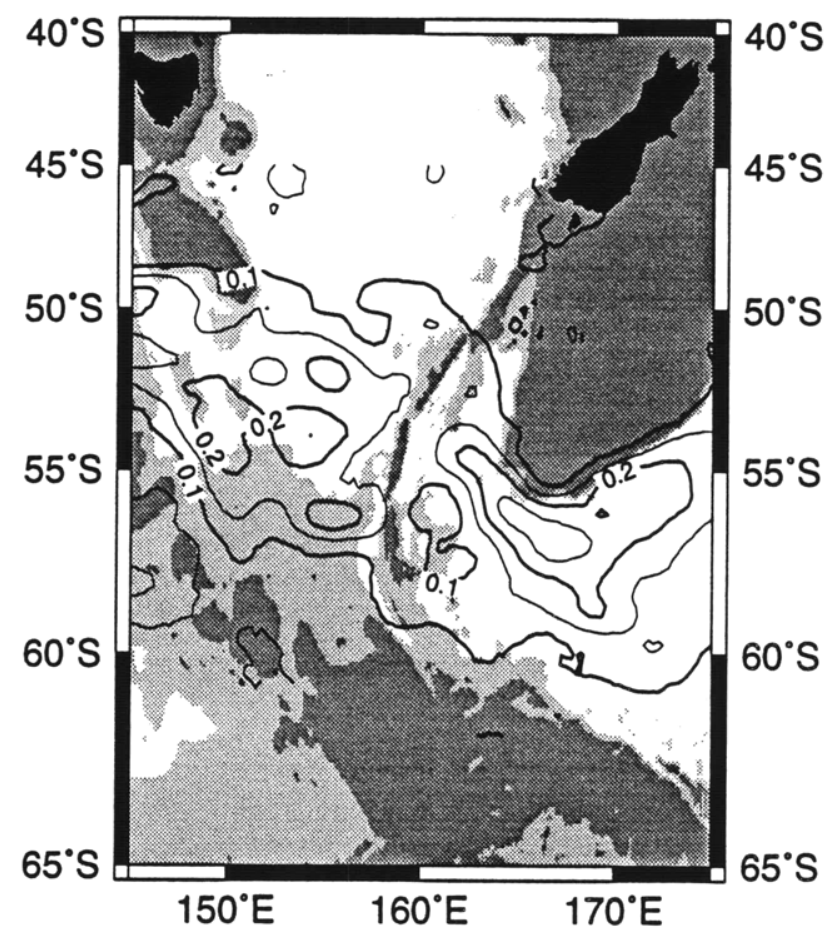

Figure 3.7: Root mean squared sea surface height (in $\mathrm{m}$ ) south of Australia and New Zealand. The contour interval is $0.05 \mathrm{~m}$.

quarie Ridge Complex, and around the Campbell Plateau. In this analysis the ACC is assumed to contain two meandering fronts, the Subantarctic Front (SAF) and the Polar Front (PF), roughly separated by the 0 height contour. Although the resolution of the mean sea surface height does not sharply distinguish the two fronts, the mean map suggests that the topographic rise at $148^{\circ} \mathrm{E}, 53^{\circ} \mathrm{S}$ (indicated with an arrow) separates the SAF and the PF. In the Macquarie Ridge Complex, the SAF passes to the north of Macquarie Island, (around $160^{\circ} \mathrm{E}, 55^{\circ} \mathrm{S}$ ) and the PF to the south.

The rms sea surface height in Figure 3.7 indicates that the mean flow is concentrated in regions of elevated variance. The variance is greatest south of New Zealand, where the ACC jets are steered around Campbell Plateau, and appears particularly large just downstream of the two gaps in the Macquarie Ridge system through which the ACC fronts appear to flow. 
Instantaneous eddy fields, objectively mapped onto a quarter-degree grid, are added to the mean sea surface height to produce a time sequence (and error map) illustrating eddy processes in the Southern Ocean (Figure 3.8). The time series indicates two continuous meandering jets which stretch across the domain, superimposed on an energetic background eddy field. Both the SAF and PF meander substantially as they travel southeast from $145^{\circ} \mathrm{E}, 50^{\circ} \mathrm{S}$ to $165^{\circ}, 58^{\circ} \mathrm{S}$, and then northeast around Campbell Plateau. Nonetheless, rings and ring formation processes are clearly a significant portion of total eddy variability. Results in chapter 2 indicated that meandering accounted for less than half of the rms sea surface height in eddy active regions downstream of topography. The time sequence shown here provides anecdotal evidence to corroborate the statistics. From the first image to the last, we see evidence of a meander, at $157^{\circ} \mathrm{E}, 57^{\circ} \mathrm{S}$ (indicated by the lower arrow in Figure 3.8a) gradually elongating, breaking off, and moving away from the Polar Front. Just to the north of that feature, at $157^{\circ} \mathrm{E}, 53^{\circ} \mathrm{S}$, a meander on the northern side of the PF (indicated by the upper arrow in Figure 3.8a) separates from the front to form a coherent ring and finally coalesces into the center of a SAF meander, suggesting a mechanism by which eddy processes may directly transport water masses across the Polar Frontal Zone.

These images provide a qualitative picture of strong mesoscale variability. In the next section, statistical techniques will be used in an attempt to quantify the large-scale variability of the ACC.

\subsection{Seeking Coherence in Variability}

\subsubsection{Time Domain Variability}

Empirical orthogonal functions (EOFs) offer a simple means to examine the dominant modes of variability in an oceanographic data set [Davis, 1976]. They provide the 

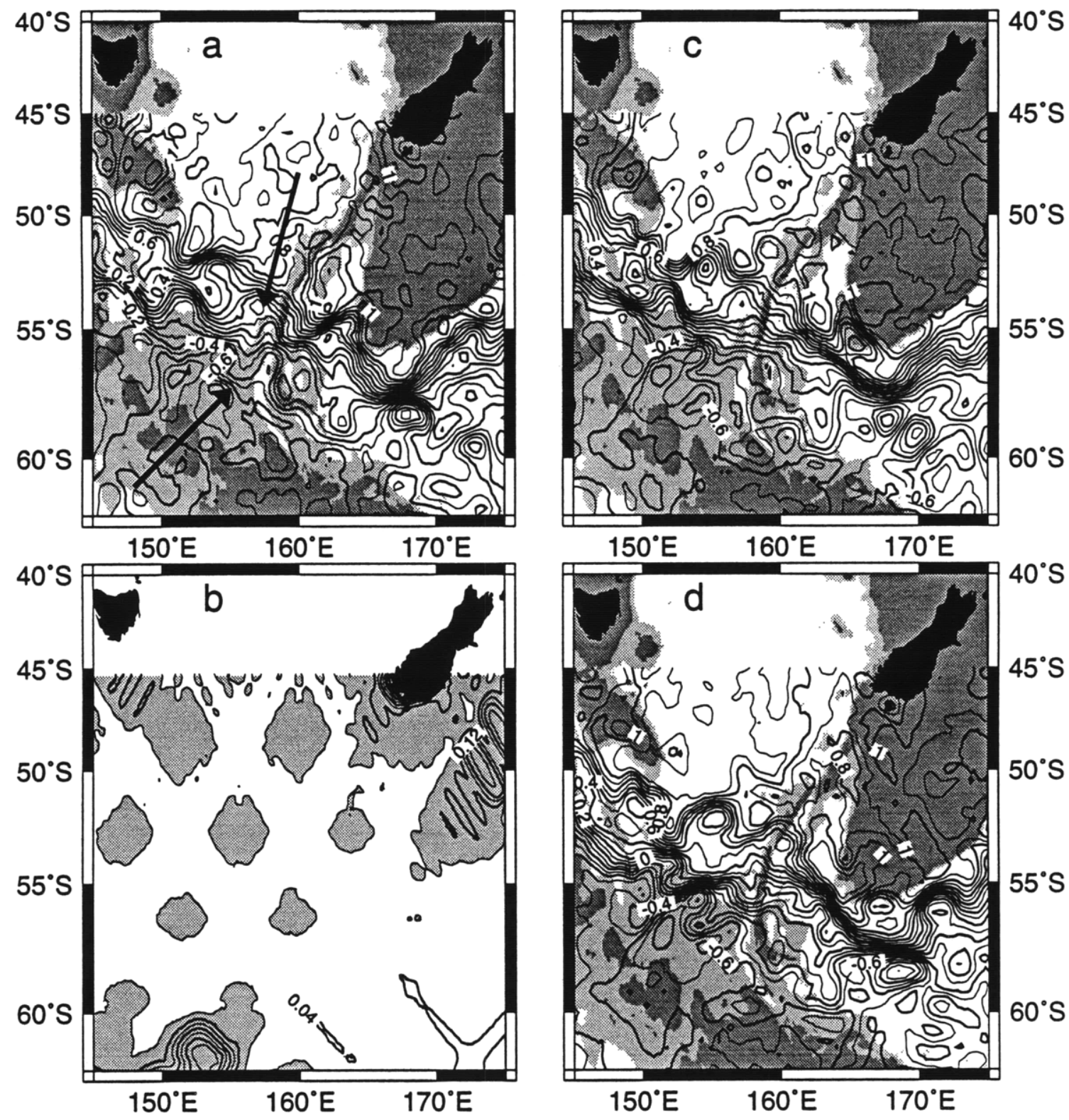

Figure 3.8: (a) Instantaneous sea surface height south of Australia and New Zealand for April 9, 1987, reconstructed by adding the mean field in Figure 3.6 to the objectively mapped instantaneous height. Sea surface height is in meters and is contoured with a $10 \mathrm{~cm}$ contour interval. Results are superimposed on ETOPO5, 5 minute resolution bathymetric data. Dark gray regions are shallower than $3000 \mathrm{~m}$, and lighter gray regions shallower than $4000 \mathrm{~m}$. (b) Error map for instantaneous sea surface height shown in a. Most of the region has errors between 5 and $7 \mathrm{~cm}$; gray regions indicate errors greater than $6 \mathrm{~cm}$. The contour interval is $2 \mathrm{~cm}$. Errors are particularly large over Campbell Plateau, where only ascending orbit data is used. (c) Instantaneous sea surface height for April 26, 1987. (d) Instantaneous sea surface height for May 13, 1987. 
statistical machinery necessary to examine whether the ACC fluctuates coherently in response to seasonal wind forcing or any other temporally varying effects.

In this analysis, EOFs are computed on the variations in total surface transport in the ACC jets as a function of time and longitude. The time series of sea surface height difference across the ACC, for each of the 244 tracks, at 63 steps in time were Fourier transformed to the frequency domain using a technique which accounts for the data gaps in the time series [Ferraz-Mello, 1981]. Ascending and descending tracks were treated as separate datasets because of the complications associated with merging information from intersecting tracks when the latitude of the current varies substantially as a function of longitude. On the global scale, the results for ascending and descending data are similar; only results from descending tracks will be plotted since they suffer from fewer data gaps. Figure 3.9 shows wavenumber and frequency spectra for all of the surface transport estimates. A boxcar-hanning window was applied to exclude all but the lowest 15 frequencies, corresponding to about 5 cycles per year, where the spectrum is red, and the data were inverse transformed to produce a complete time-space series of sea surface height differences. Although the wavenumber spectrum is somewhat white, with a peak around 40 cycles per $360^{\circ}$, it indicates that the greatest energy is at low wavenumbers. The data were therefore spatially filtered, retaining roughly the lowest 20 zonal wavenumbers, corresponding to about $18^{\circ}$ longitude or $1000 \mathrm{~km}$ in order to examine large-scale phenomena.

EOFs were computed using a singular value decomposition [Kelly, 1988]. In the results presented, each EOF mode represents coherent changes in sea surface height as a function of time; the amplitude of the mode varies spatially but the phase is globally constant. Unlike other orthogonal functions, such as sines and cosines, the major virtue of EOFs is that they are directly tied to the data statistics. The first mode EOF captures the largest percentage of the variance which can be explained by coherent variations in surface transport, and the remaining EOFs are sorted according to decreasing variance represented. Thus, if the dominant fluctuations in ACC transport 

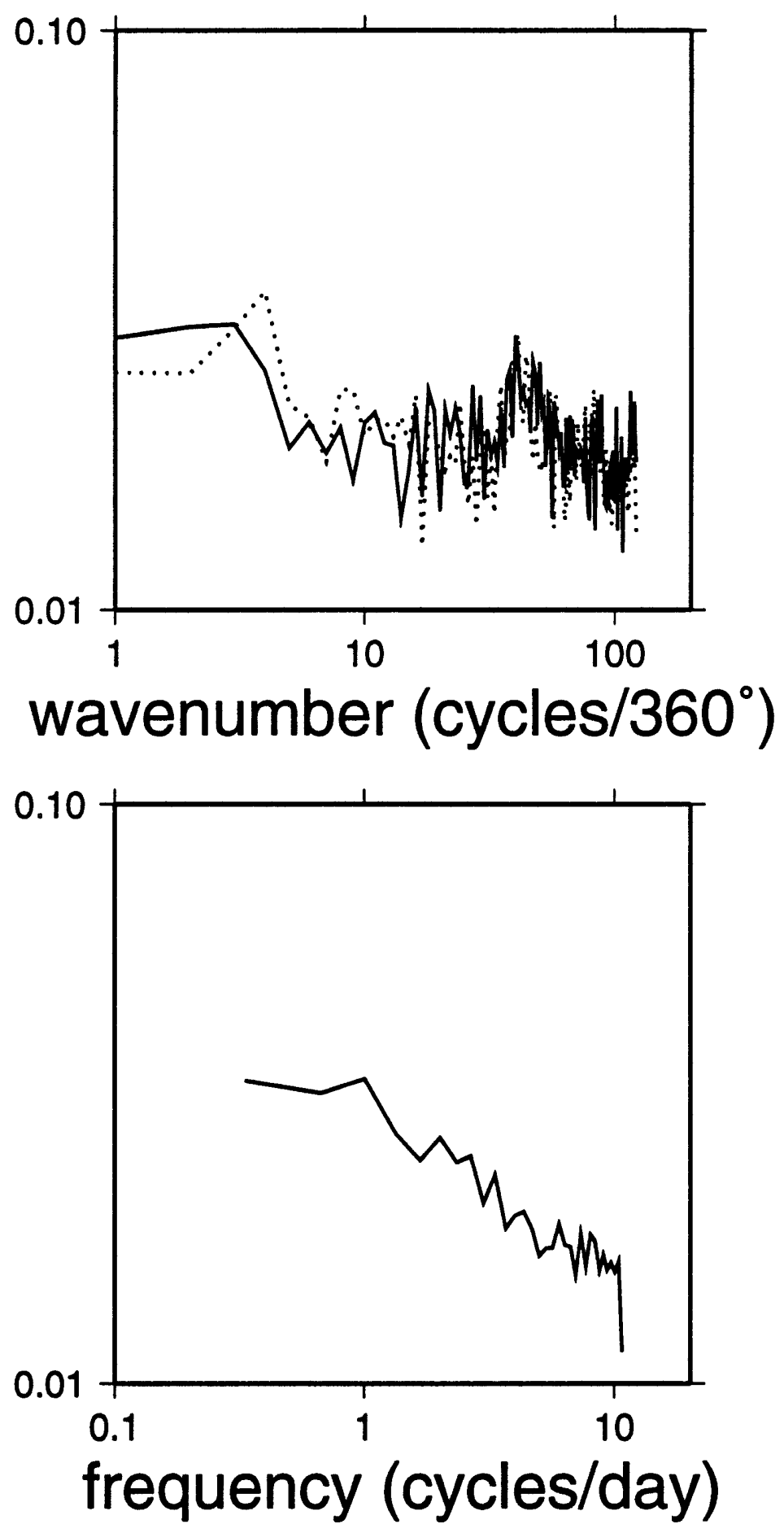

Figure 3.9: (Top) Wavenumber spectrum for surface transport variability for ascending and descending track data, combined. (Bottom) Frequency spectrum for surface transport variability for ascending and descending track data, combined. Values shown represent the power spectral density (in $\mathrm{m}^{2}$ ). 
were globally coherent, as Wearn and Baker [1980] suggested, then at all longitudes the first mode EOF would have roughly the same amplitude and would account for about the same fraction of variance. Conversely, if the leading mode EOFs explain little of the variance and are not global in structure, then we conclude that the dominant fluctuations in ACC transport occur on smaller spatial scales.

\section{EOFs for the Full Southern Ocean}

The first EOF for the entire Southern Ocean domain accounts for $16.5 \%$ of the variance in descending track data and $16.6 \%$ in ascending track data. The spatial structure of the mode does not show large-scale structures, and the fraction of variance explained as a function of longitude (Figure 3.10) indicates that most of the mode's effect is concentrated in a few locations. None of the higher order modes are any more suggestive of larger scale structure in the variability. In the time domain (Figure 3.11), the first mode EOF fluctuates on roughly a semi-annual (180 day) time scale, but without a single well-defined frequency. Higher order temporal modes do not clearly correspond to annual or semi-annual time scales which might be interpreted as a response to wind forcing. The magnitude and timescale of first mode EOF fluctuations are comparable to the variations seen by Wearn and Baker [1980], suggesting that the Drake Passage variability measured by pressure gauges may be typical of the full Southern Ocean, even if the overall response is not coherent.

Significance levels were tested using Rule N outlined by Preisendorfer [1988]. This is a Monte Carlo technique, in which EOFs are computed for 100 sets of white noise which have been filtered and treated in the same way as the raw data, producing 100 sets of eigenvalues, $l_{j}$, where $j$ goes from 1 to $n$, the number of data eigenvectors. By numerically sorting the 100 eigenvalues at each $j$, and selecting the 5th and 95th values, $90 \%$ significance bounds are determined; those eigenvalues which exceed the upper limit are interpreted to represent eigenmodes which are statistically different from white noise. 


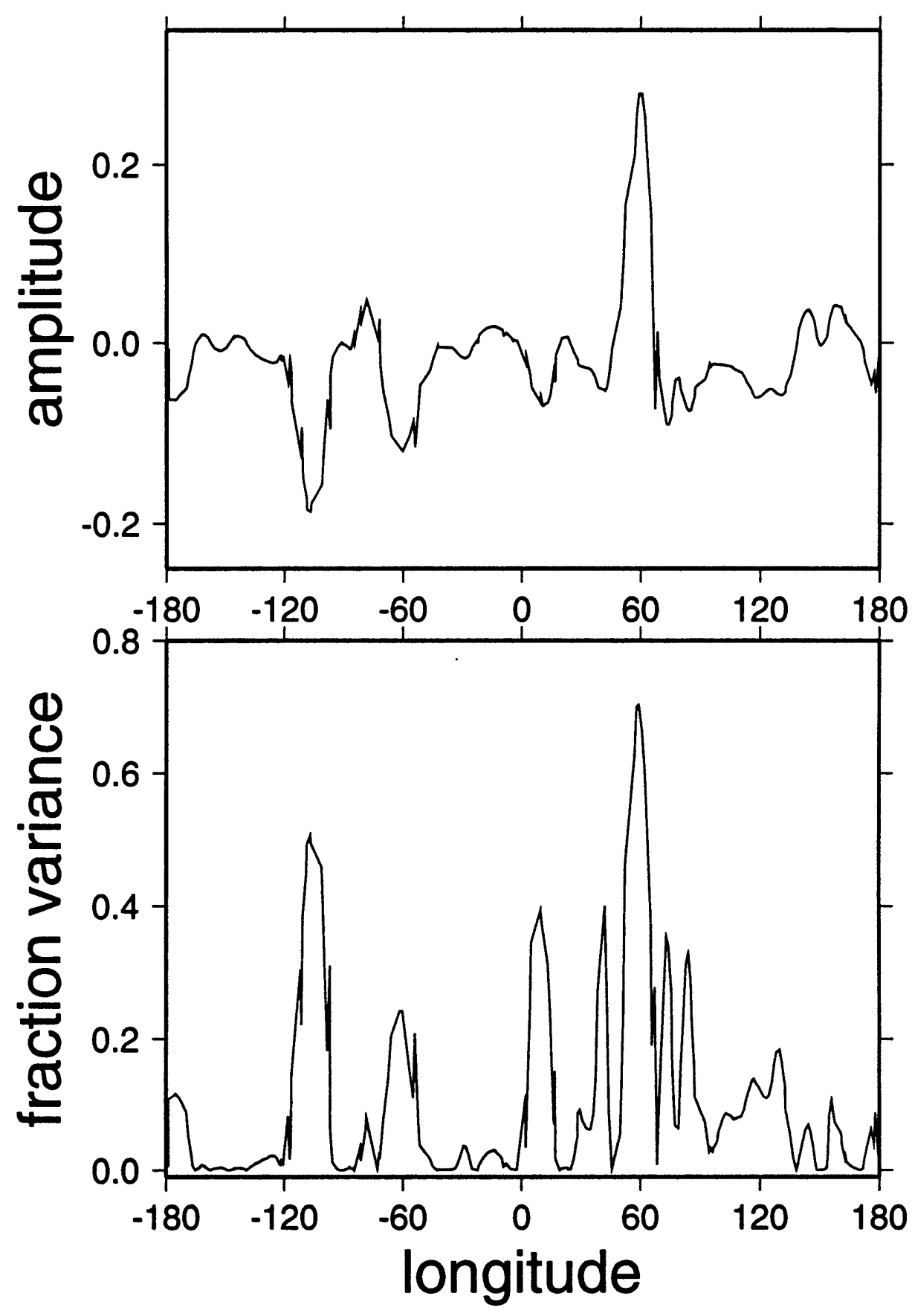

Figure 3.10: (Top) Spatial structure of first mode EOF for all descending track data (in $\mathrm{m}$ ). (Bottom) Fraction of variance explained by the first mode EOF as a function of longitude. 


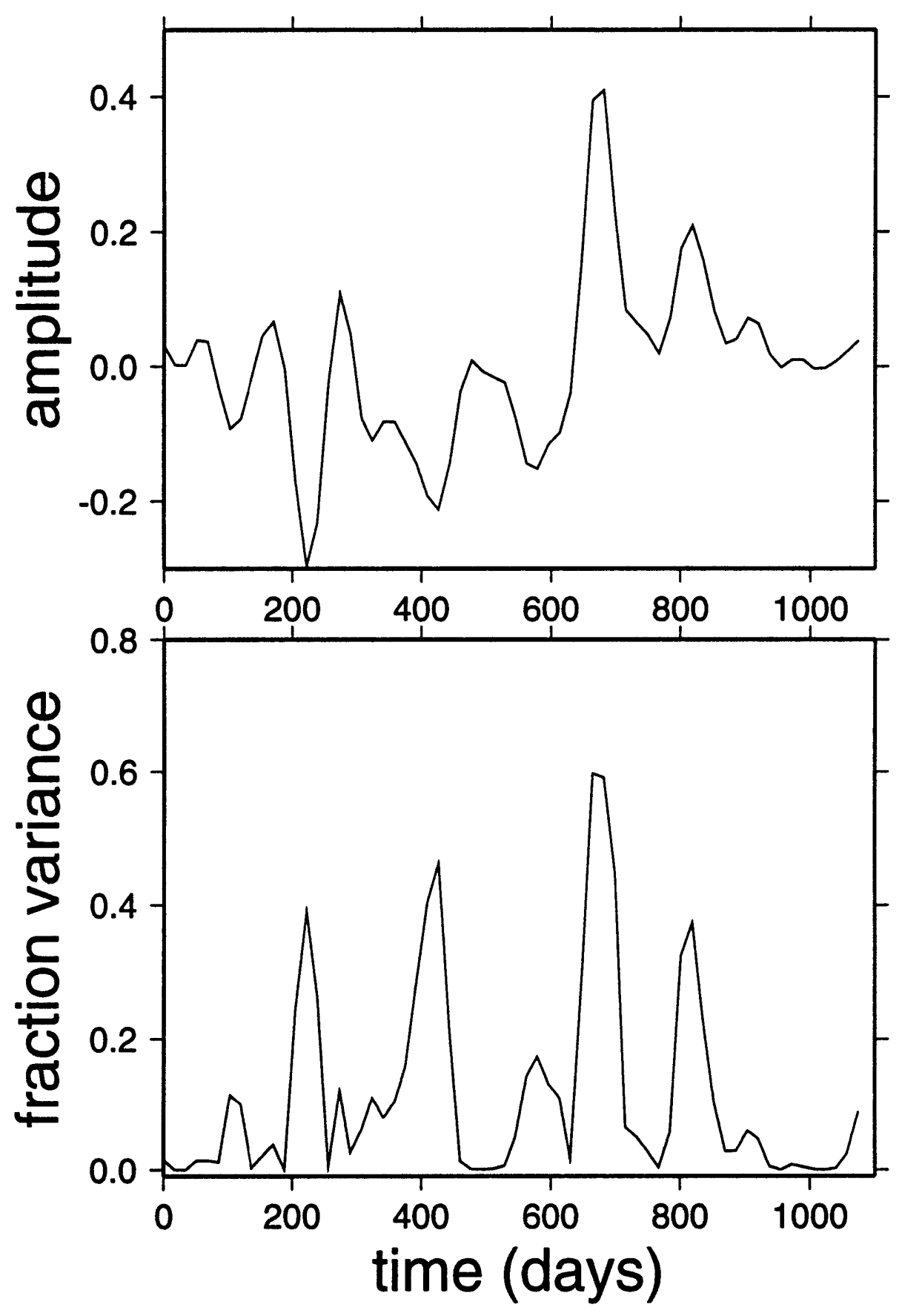

Figure 3.11: (Top) Temporal structure of first mode EOF for all descending track data (in $\mathrm{m}$ ). (Bottom) Fraction of variance explained by the first mode EOF as a function of time. 


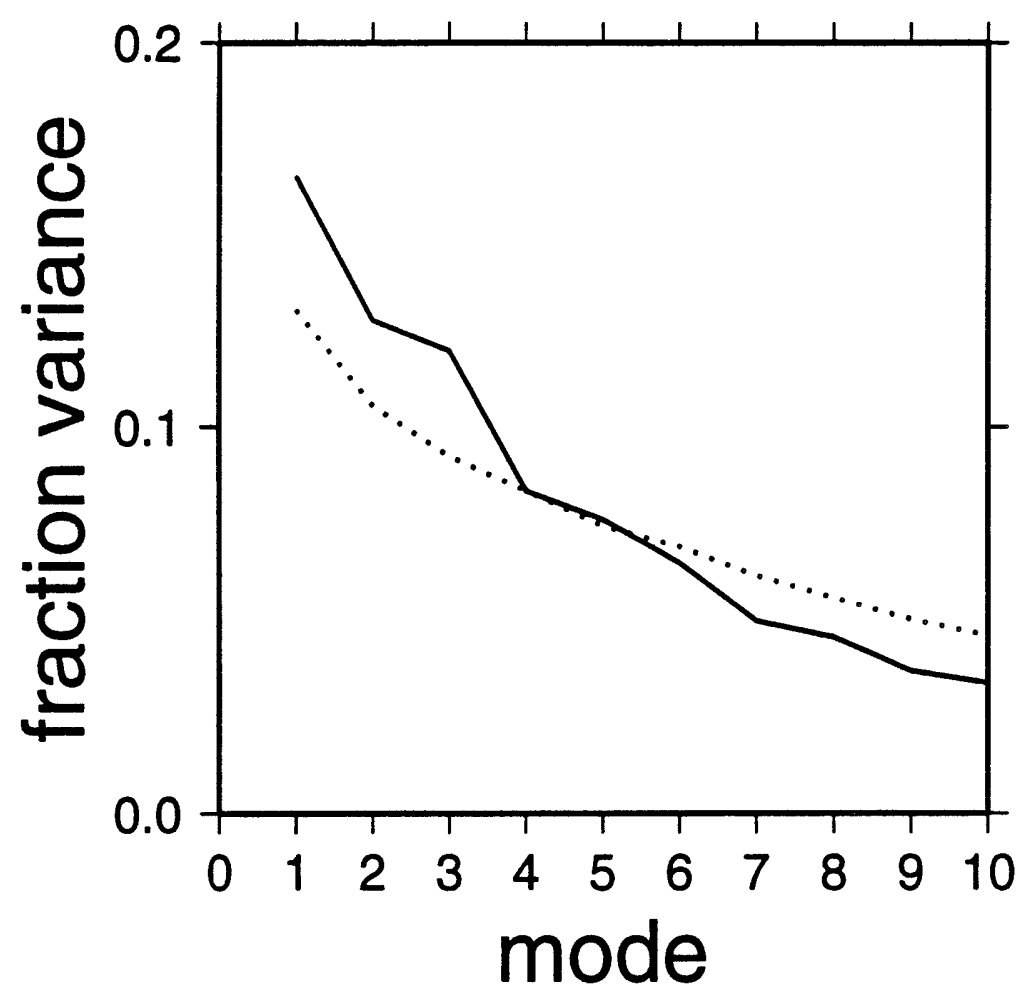

Figure 3.12: (Solid line) Percentage of variance explained by the first 10 EOF modes for descending track data, and (dotted line) $N$-test values indicating the $90 \%$ significance limits for EOFs computed on pure white noise. Only EOF modes exceeding this significance limit are judged to be statistically meaningful. See text.

Based on Rule N, the first 5 modes of the Southern Ocean EOF analysis are statistically significant (Figure 3.12). Together these 5 modes account for $57 \%$ of the variance of descending tracks and $56 \%$ for ascending tracks. Thus over $40 \%$ of the variance is not described by coherent variability. The low percentages of variability represented by the first few EOFs are comparable to the results found by Chelton et al. [1990] in a computation of EOFs for filtered and gridded sea surface height variability data at Geosat crossover points in the Southern Ocean; they found that the first EOF accounted for only $15 \%$ of the global variance. Although the data shown in this analysis represent only surface transport within the ACC jets and do not account for any large-scale background flow or recirculation, the close quantitative match with Chelton's results suggests that the EOF modes may capture representative processes 
in the Southern Ocean. These results imply that dominant Southern Ocean variability is not global in scale.

\section{Basin Scale and Regional EOFs}

If the Southern Ocean does not undergo system-wide fluctuations, it may still be coherent on a basin scale, with regional flow patterns broken up by the topographic features which separate it into basins and which are associated with substantial eddy variability. Indeed, separating the flow into basins shows a substantial increase in the percentage of the variance accounted for by the first few EOFs. Chelton et al. [1990] found that first mode EOFs explained $26 \%, 21 \%$, and $22 \%$ of the variability in the Atlantic, Indian, and Pacific sectors, respectively. In this analysis, a number of different basin divisions of varying sizes were examined, using both continental margins and bathymetric features to define the basin limits. The first mode EOFs account for between $20 \%$ and $35 \%$ of the variability, depending on the basin considered and the precise boundaries of the basin, as indicated in Table 3.1.

Substantial differences between ascending and descending tracks are not surprising because of differences in the orientation of the tracks and the number of data points available in each ocean basin. Since the basin scale variability was very small, a number of smaller regions were also considered. Rule $\mathrm{N}$ tests indicate that a maximum of 2 modes are statistically significant at the $90 \%$ level, as illustrated for several examples in Figure 3.13. Overall these results are consistent with Chelton et al.'s results, and suggest that Southern Ocean variability may be better described by basin scale motions.

The full interpretation of these modes, however, can be extremely deceptive. In any statistical system, reducing the number of degrees of freedom (in this case, the number of sea surface height locations considered) will necessarily increase the magnitudes of some of the eigenvalues. When a smaller region is considered, we must ask not simply whether the percent variance described by the first few modes increases, 


\begin{tabular}{|ll|ccc|}
\hline Region & orientation & $\%$ variance & N modes & $\begin{array}{c}\text { N modes } \\
\text { (reduced degrees } \\
\text { of freedom) }\end{array}$ \\
\hline Southern Ocean & ascending & $16.6 \%$ & 5 & \\
Atlantic Ocean & descending & $16.5 \%$ & 5 & \\
$52^{\circ} \mathrm{W}-70^{\circ} \mathrm{E}$ & ascending & $26.3 \%$ & 2 & 0 \\
& descending & $38.4 \%$ & 2 & 1 \\
$70^{\circ} \mathrm{W}-25^{\circ} \mathrm{E}$ & ascending & $35.0 \%$ & 2 & 1 \\
& descending & $36.6 \%$ & 2 & 1 \\
Indian Ocean & & & & \\
$70^{\circ} \mathrm{E}-170^{\circ} \mathrm{E}$ & ascending & $26.4 \%$ & 0 & 0 \\
& descending & $33.1 \%$ & 1 & 1 \\
$25^{\circ} \mathrm{E}-170^{\circ} \mathrm{E}$ & ascending & $21.4 \%$ & 0 & 0 \\
& descending & $29.0 \%$ & 2 & 2 \\
Pacific Ocean & & & & 0 \\
$170^{\circ} \mathrm{E}-52^{\circ} \mathrm{W}$ & ascending & $23.4 \%$ & 1 & 1 \\
& descending & $25.5 \%$ & 2 & 0 \\
$170^{\circ} \mathrm{E}-70^{\circ} \mathrm{W}$ & ascending & $19.5 \%$ & $2^{*}$ & 1 \\
Central Pacific & descending & $30.4 \%$ & 1 & 0 \\
$170^{\circ} \mathrm{W}-110^{\circ} \mathrm{W}$ & ascending & $34.2 \%$ & 0 & 0 \\
Central Indian & descending & $34.5 \%$ & 1 & 0 \\
$42^{\circ} \mathrm{E}-101^{\circ} \mathrm{E}$ & ascending & $31.6 \%$ & 0 & 1 \\
Eastern Atlantic & descending & $49.5 \%$ & 1 & 0 \\
$10^{\circ} \mathrm{W}-50^{\circ} \mathrm{E}$ & ascending & $33.0 \%$ & 0 & 0 \\
& descending & $26.6 \%$ & 0 & 0 \\
\hline
\end{tabular}

Table 3.1: Percentage variance explained by first mode EOF for basin scale regions of the Southern Ocean. Ocean basins are defined first by geographic features-Drake Passage, Kerguelen Island, and Campbell Plateau-and second by continental divisions. The column labeled "N modes" indicates the number of EOF modes estimated to be significant at the $90 \%$ level based on an $\mathrm{N}$ test (described in the text). In the case marked with $a^{*}$, the first mode is not significant, but the second and third are. The column labeled "N modes (reduced degrees of freedom)" indicates the number of modes estimated to show a statistically significant increase from the basin scale values (see text). 

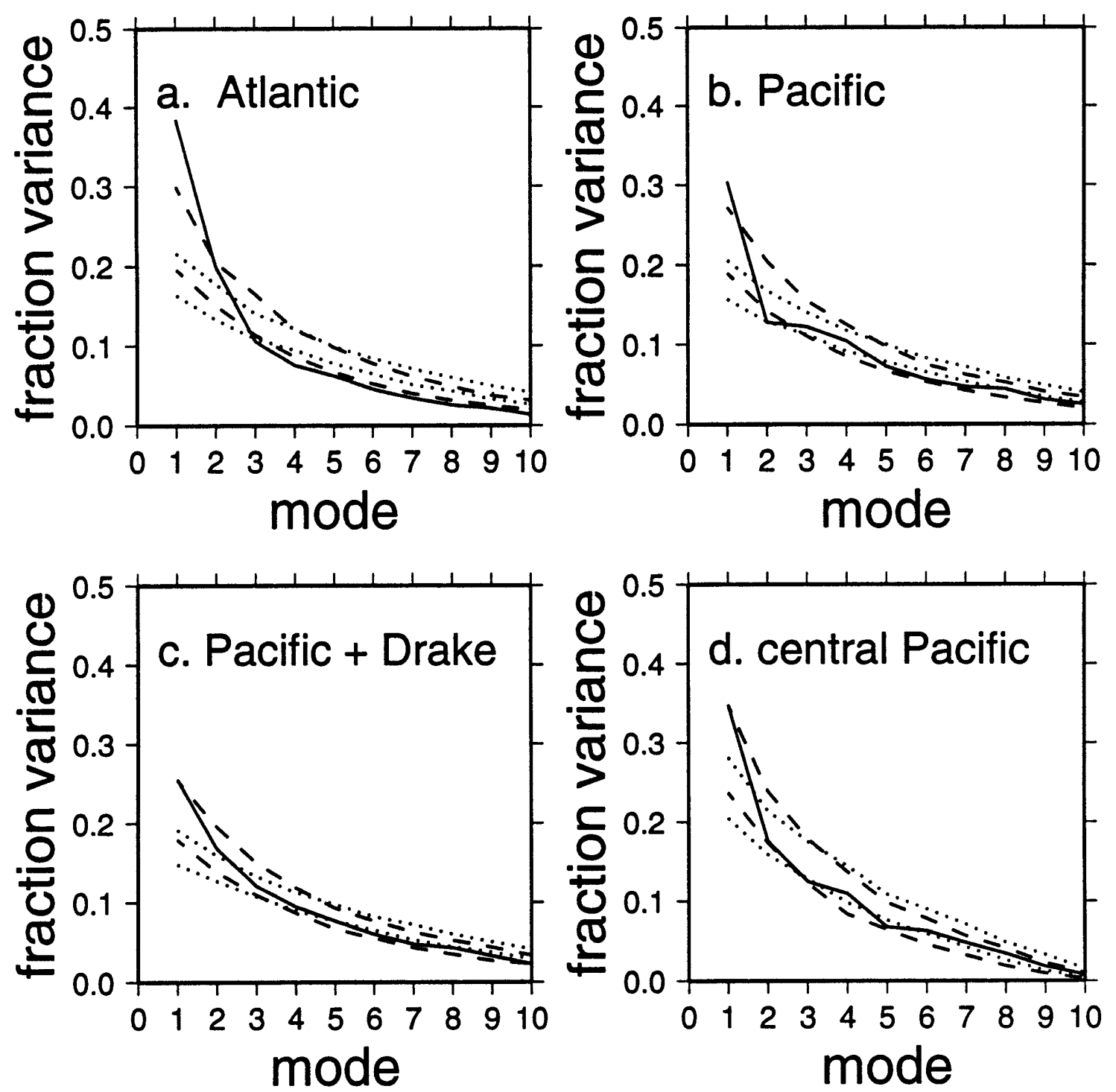

mode

Figure 3.13: (Solid lines) Fraction of variance explained by first 10 EOFs for four regions in the Southern Ocean (solid lines): (a) Atlantic Ocean defined by bathymetry from Drake Passage to Kerguelen Island ( $70^{\circ} \mathrm{W}-25^{\circ} \mathrm{E}$ ); (b) Pacific Ocean defined by continents $\left(170^{\circ} \mathrm{E}-70^{\circ} \mathrm{W}\right) ;$ (c) Pacific Ocean defined by bathymetry from Campbell Plateau through Drake Passage; (d) central Pacific Ocean $\left(170^{\circ} \mathrm{W}-119^{\circ} \mathrm{W}\right)$. Significance levels are (dotted lines) determined from $\mathrm{N}$ tests performed on filtered white noise and (dashed lines) the relative significance of reducing the number of degrees of freedom (see text). 
but more specifically, whether it increases by a statistically significant margin, given the reduction in the degrees of freedom. To test precisely this issue, synthetic data were created for the Southern Ocean: Gaussian white noise was filtered, subsampled, and decomposed to compute EOFs in the same way as the original sea surface height measurements. The eigenvectors determined from white noise were retained, but eigenvalues were then fixed to correspond to the eigenvalues of the filtered data for the entire Southern Ocean. Thus, the relative importance of the modes in the synthetic data is the same as in the real data, but the spatial structure of the modes is random and has no link to physical features within the ocean. When the number of samples used was reduced to look at basin scale variability, the ensemble of eigenvalues for the synthetic data was sorted and interpreted to indicate $90 \%$ significance levels. This procedure is analogous to the more conventional Rule $\mathrm{N}$ outlined above. Only if the real EOFs exceed the $90 \%$ limits can they be interpreted as indicating significant basin scale motions.

Although Rule $\mathrm{N}$ indicated that as many as 2 EOFs were statistically significant, considerations of the reduced degrees of freedom (dashed lines in Figure 3.13) indicated that these modes were only marginally significant, and often no modes were significantly different from their global scale equivalents. In other words, the apparent increase in the variance explained by the first mode EOF was largely an artifact of reducing the size of the data set. These tests suggest that Southern Ocean variability is not characterized by large single modes sloshing up and down, either on the global or basin scale.

The geographic distribution of the variance explained by the EOFs supports this conjecture. The first mode EOF for the entire Southern Ocean (Figure 3.10) explains almost all of the variance in isolated $1000 \mathrm{~km}$ long regions typically associated with topography, such as the Eltanin-Udintsev Fracture Zone in the middle of the Pacific $\left(130^{\circ} \mathrm{W}\right)$. Since the spatial filter eliminates variability on spatial scales less than about $1000 \mathrm{~km}$, the dominant EOF modes cannot capture mesoscale features, 
but they are clearly not associated with basin or global scale variability. Figure 3.14 shows that even when the basin scale and smaller regions of the Southern Ocean are considered, the first mode EOF does not describe basin wide variability; instead its effect is confined to smaller regions. Regions where the first mode EOF describes most of the variance are associated with topographic features and have greater eddy variability. For the regions shown in Figure $3.14,60^{\circ} \mathrm{E}$ in the Atlantic is downstream of the Crozet Plateau, and $110^{\circ} \mathrm{W}$ in the Pacific is downstream of the Eltanin-Udintsev Fracture Zone. While variance weighted EOFs have a slightly different structure, they also indicate no basin scale coherence and suggest that variability occurs on length scales of no more than $1000 \mathrm{~km}$. Thus all evidence from this time domain EOF analysis suggests that the Southern Ocean does not show a large scale coherent response to wind forcing.

\subsubsection{Frequency Domain Variability}

Since surface transport in the Southern Ocean does not indicate a spatially stationary coherent response, we might predict that transport fluctuations instead propagate along the length of the ACC in the form of traveling waves. The frequency-wave number spectrum shown in Figure 3.15 indicates a statistically significant peak at the annual frequency with increased energy at the wavenumber corresponding to 3 cycles per $360^{\circ}$ propagating westward. The next largest signal propagates eastward with a frequency of 0.33 cycles per year and wavenumber 3 cycles per $360^{\circ}$.

Time domain EOFs do not capture the propagating variability indicated in the frequency-wavenumber spectrum, but frequency domain EOFs provide a means to determine dominant traveling wave modes [Preisendorfer, 1988]. Therefore frequency domain EOFs were also considered. However, computing EOFs in the frequency domain reduces the number of degrees of freedom in the data, and with that limitation, no statistically significant traveling wave modes emerged in the analysis. 

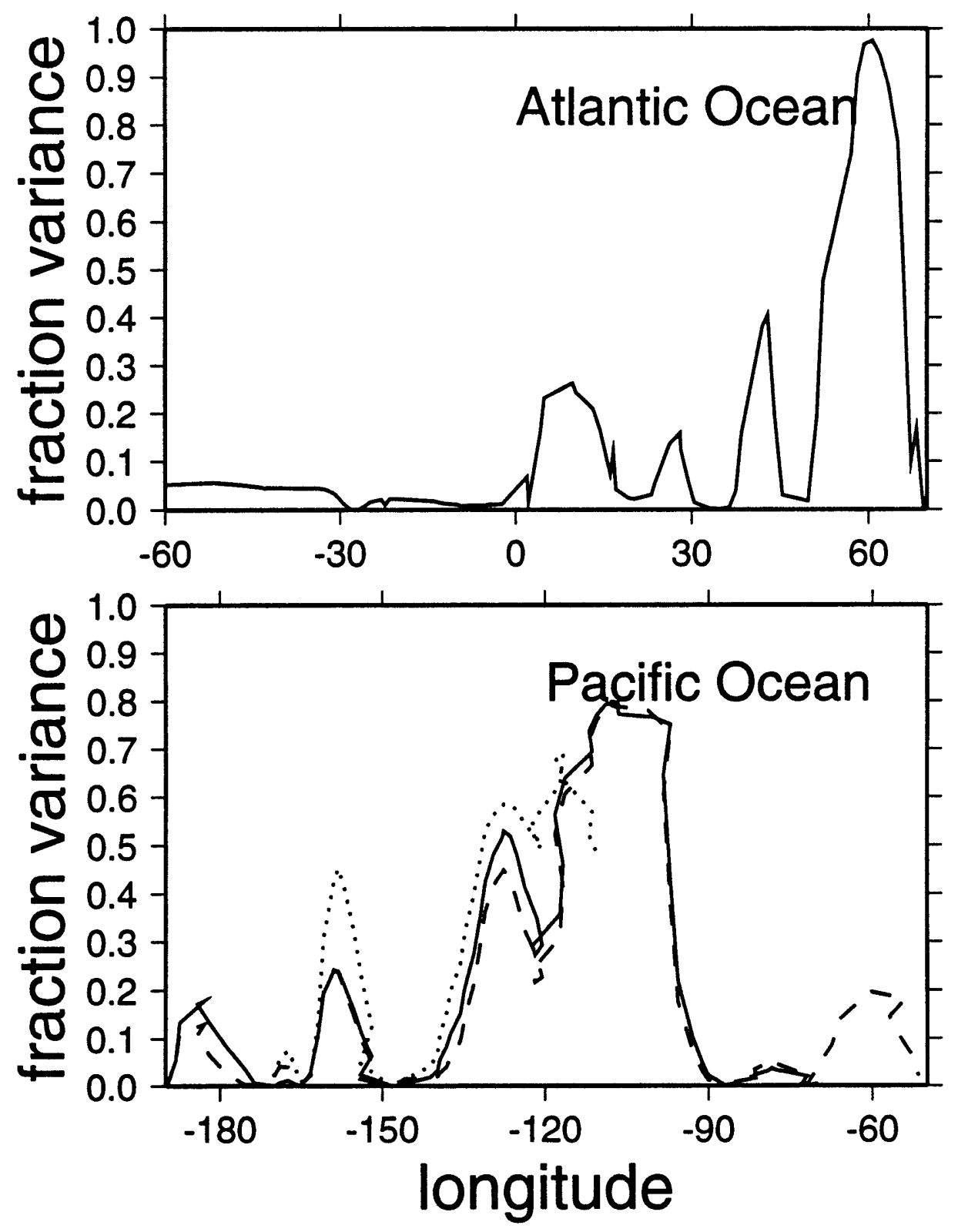

Figure 3.14: Fraction of variance explained by first EOF as a function of longitude for four regions in the Southern Ocean: (top) Atlantic Ocean defined by bathymetry from Drake Passage to Kerguelen Island $\left(70^{\circ} \mathrm{W}-25^{\circ} \mathrm{E}\right.$ ); (bottom) Pacific Ocean defined by (solid line) continents $\left(170^{\circ} \mathrm{E}-70^{\circ} \mathrm{W}\right)$, (dashed line) Pacific Ocean defined by bathymetry from Campbell Plateau through Drake Passage, (dotted line) central Pacific Ocean $\left(170^{\circ} \mathrm{W}-119^{\circ} \mathrm{W}\right)$. Results indicate no clear basin wide structure. 


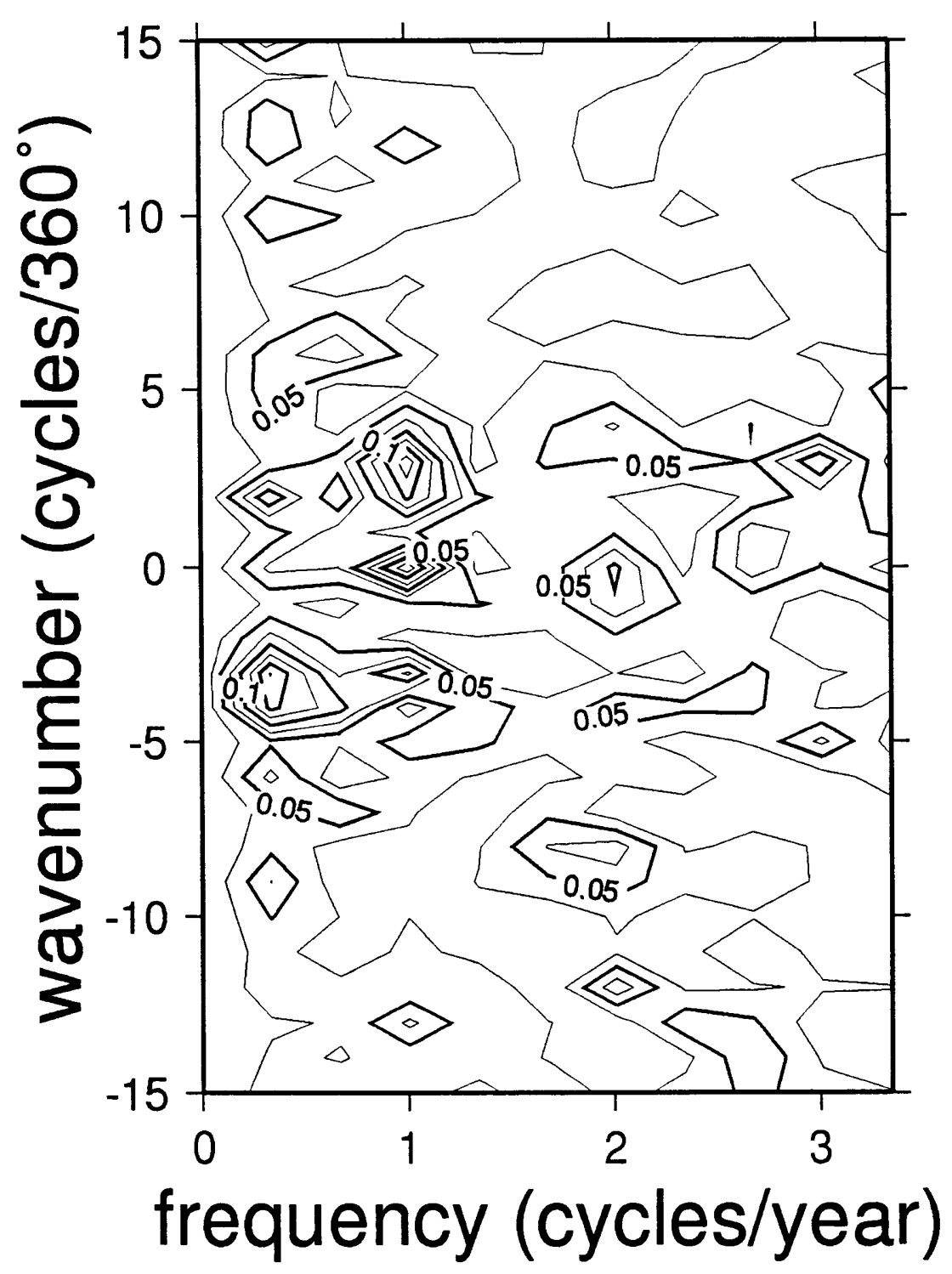

Figure 3.15: Frequency-wavenumber spectrum for surface transport variability for ascending and descending track data, combined. Values shown represent the power spectral density (in $\mathrm{m}^{2}$ ). The mean signal as a function of time is removed, so that there is no energy at zero frequency. The peak amplitude has an annual frequency and a wavenumber of +3 cycles per $360^{\circ}$ with westward propagation. Amplitudes exceeding $0.05 \mathrm{~m}^{2}$ (those enclosed by dark lines) are statistically significant at the $95 \%$ level based on Monte Carlo results taking into account irregularities in the data sampling. 
In addition, although the spectrum appears to indicate strong propagating waves, a nonlinear least-squares fit of the data to a traveling wave mode did not explain a significant percentage of the variance. As the broad energy peaks in the spectrum would suggest, no single frequency and wavenumber captures a significant portion of the total variance.

\subsection{Discussion: Interpreting Incoherence}

These considerations of Southern Ocean surface height fluctuations using EOFs suggest that the dominant scales of variability range from meso to $1000-\mathrm{km}$ scale, and that large scale motions do not clearly propagate through the system. The results are generally consistent with in situ observations. An analysis of surface drifters indicated no global scale structure in variability and no coherence between wind and ocean variability [Large and van Loon, 1989]. Likewise, limited comparisons of pressure gauge measurements in Drake Passage and between Amsterdam and Kerguelen Islands provided no evidence of global scale structure in Southern Ocean variability and no particular annual or semi-annual harmonic in ocean fluctuations [Vassie et al., 1994]. Several mechanisms may account for the observed lack of coherence in variability.

First, despite well-known observations emphasizing the semi-annual cycle in the winds over the Southern Ocean [van Loon, 1972], enormous uncertainties remain in the general structure of the fields south of $30^{\circ} \mathrm{S}$. The ECMWF model provided the best wind estimates for the Geosat time period, but in a detailed comparison with 1978 scatterometer data, Mestas-Nunez et al. [1994] concluded that ECMWF wind fields were highly suspect over the core of the ACC. ECMWF winds indicate high interannual variability over the major wind belts, although in the ACC the dominant fluctuations occur on annual and semi-annual time scales and are global in scale. The 
results of Mestas-Nuñez et al. suggest that the actual winds may vary much more substantially and on a broader range of scales than the ECMWF fields indicate.

Other measurements also underscore the lack of a strong seasonal cycle in the Southern Hemisphere. In an analysis of global TOPEX/POSEIDON altimeter measurements, Stammer and Wunsch [1994] found that seasonal changes in sea surface height due to heat and salinity fluctuations were much smaller in the Southern Hemisphere than in the Northern Hemisphere. Gill and Niiler [1973] estimated that most of the seasonal sea surface height variability is due to atmospheric pressure loading and to steric changes in the upper ocean, which they attributed largely to temperature fluctuations rather than salinity changes. Thus the small seasonal signal for sea surface height in the Southern Ocean implies that the system does not experience strong periodic buoyancy forcing and that sea surface temperature does not undergo a large annual cycle and is therefore unlikely to provide strong feedback to the atmosphere to drive substantial globally coherent surface winds. While weak annual variability in temperature does not preclude a globally coherent wind response in the ACC, the temperature cycle which drives seasonal winds is global in nature. Thus we might conjecture that winds due to anything other than seasonal temperature cycles are likely to vary geographically and will not coherently force the ACC. Though the wind and sea surface height fluctuations may include a small seasonal component in response to global scale changes in solar forcing, the dominant variability captured by the EOFs occurs on mesoscale to $1000-\mathrm{km}$ spatial scales.

Other factors may prevent the ACC from responding in a globally coherent fashion. Along its convoluted circumpolar path, the ACC passes over numerous topographic features which generate form stress, removing momentum input by surface winds. These ridges are associated with substantial mesoscale eddy activity and instability processes which may break up the flow of the ACC and interrupt any large scale coherent structures. Although numerical model results indicate that form stress occurs predominantly at three major topographic features (see chapter 4 ) the lack of 
large scale coherent oceanic response, even on basin scales suggests that many more topographic features could be sources of form drag.

Finally, limitations in the altimeter measurements may conceal the oceanic response to wind forcing. First, the mesoscale analysis technique used in this study captures variability in the jets, but does not measure any possible response to wind forcing on a broader meridional scale. However, since most of the ACC transport is contained within the narrow jets and large scale analyses by Chelton et al. [1990] and Vassie et al. [1994] have not indicated globally coherent response, the mesoscale analysis appears unlikely to be a major limitation. Second, the altimeter measures fluctuations in surface transport, but is unable to consider how the baroclinic structure of the flow may change with varying wind forcing. Depending on stratification conditions, the rate at which the ACC spins up will differ, so that the flow may deepen in response to wind forcing, increasing total transport without substantially changing surface transport. Southern Ocean observations have not provided a clear picture of the vertical structure of ACC variability. Pressure gauge measurements in Drake Passage suggested that transport fluctuations are largely barotropic [Whitworth and Peterson, 1985]; however Vassie et al. [1994] argued that the annual cycle measured by pressure gauges at Amsterdam and Kerguelen Islands could be partly due to baroclinic changes not seen by the altimeter. (More recent work with TOPEX/POSEIDON data and improved tidal models has shown close agreement between the Amsterdam Island pressure gauge and altimeter measurements [Park and Gambéroni, 1994], which might suggest that baroclinic effects may not be a significant factor in the annual variability.)

Ultimately more detailed observations may be necessary to determine whether substantial spatio-temporal variability in the wind field, topographic forcing, or baroclinic fluctuations account for the lack of coherent structure in this analysis of ACC surface transport. Forthcoming scatterometer measurements of surface winds and on- 
going TOPEX/POSEIDON altimeter measurements will undoubtedly provide some illumination.

\subsection{Summary}

This work has examined the length and time scales of variability and the coherence of fluctuations in the Southern Ocean. Sea surface height variance maps indicate that the Southern Ocean is a region of intense eddy activity, and the decorrelation scales emphasize that much of this variability appears to occur on the scales less than $1000 \mathrm{~km}$. Spatial decorrelation scales are relatively short, about $85 \mathrm{~km}$, and time scales are about 34 days. Neither of these is extremely well resolved by the Geosat altimeter, but current meter data and analyses of ERS-1 altimeter data from a 3-day repeat orbit have suggested that the Geosat sampling should alias little energy into the sampled frequency-wavenumber space [Minster and Gennero, 1994].

The spatial and temporal information defines correlation functions which were used to objectively map instantaneous sea surface height. The maps illustrate the predominance of mesoscale processes in the Southern Ocean. In the region south of New Zealand, a series of maps shows a ring forming from a jet meander and breaking away from the Polar Front. Such processes appear more common in eddy intense regions associated with major topographic features and may be a major means by which water masses are transported across the ACC, as recent dynamical studies have suggested [eg. Johnson and Bryden, 1989; Marshall et al., 1993].

While eddy processes clearly dominate the sea surface height variability, past studies have predicted that the entire Southern Ocean transport should respond to large-scale variations in the wind forcing. Empirical orthogonal functions computed for the time varying surface transport suggest no coherent fluctuations on global or basin scales. Coherent variability appears confined to relatively small regions of 
no more than $1000 \mathrm{~km}$ length. This suggests that fluctuations in the wind may be dissipated locally, where they directly influence the flow, rather than acting to accelerate ACC transport globally. Likewise, frequency domain EOFs indicate no statistically significant traveling wave modes, although the reduced number of degrees of freedom make detailed analysis difficult.

\subsection{Appendix: Estimating Velocities and Errors}

Objective mapping provides a straightforward means to map scalar quantities and estimate corresponding variances, but derivatives of scalar quantities are more difficult to estimate. This appendix will briefly describe a how to estimate errors for velocities computed by simple first differences.

Geostrophic velocity at a point is estimated by computing the difference between two adjacent sea surface height values, labeled $i$ and $j$ :

$$
v=-\frac{g}{f}\left(\frac{h_{i}-h_{j}}{x_{i}-x_{j}}\right)
$$

with a corresponding error estimate:

$$
\sigma_{v}=\frac{g}{\left|f\left(x_{i}-x_{j}\right)\right|} \sqrt{\sigma_{h_{i}}^{2}+\sigma_{h_{j}}^{2}-2 \sigma_{h_{i} h_{j}}}
$$

where $\sigma_{h_{i} h_{j}}$ is the covariance between $h_{i}$ and $h_{j}$ [Taylor, 1982]. Thus, in general, error in a velocity estimate decreases with increasing spatial separation, $x_{i}-x_{j}$, but as the separation increases, the velocity represents an average over a larger distance, and may be less physically meaningful. An appropriate choice of $x_{i}-x_{j}$ should be no larger than the decorrelation scale, in which case the errors at $h_{i}$ and $h_{j}$ may be significantly correlated.

The covariance between the errors at adjacent objective mapped points may be estimated from the functional forms used for the objective mapping. The error 
variance is the same as the height variance, and the covariance may be estimated by using the same functional forms:

$$
\sigma_{h_{i} h_{j}}=C_{i j}-C_{i x} A_{x y}^{-1} C_{y j}
$$

where $C_{i j}$ is the covariance matrix between points on the output grid, $C_{i x}$ and $C_{y j}$ are matrices indicating the covariance between measured data points and output grid points, and $A_{x y}$ is the covariance matrix for the measured data points.

Thus the velocity error may be estimated as:

$$
\sigma_{v}=\frac{g \sqrt{2}}{\left|f\left(x_{i}-x_{j}\right)\right|} \sqrt{\sigma_{h}^{2}-\sigma_{h_{i} h_{j}}} .
$$




\section{Chapter 4}

\section{Momentum}

\subsection{Introduction}

In the simplest approximation, the Southern Ocean resembles a continuous channel centered around the Antarctic continent; the force balances which generally describe the dynamics of closed basins, such as the North Atlantic, do not easily transfer to this region. The boundaries of a closed basin support zonal pressure gradients, permitting net meridional flow to balance wind forcing; in the Southern Ocean, no such boundaries exist. Consider the momentum budget: A strong zonal wind stress drives the eastward flowing Antarctic Circumpolar Current (ACC), but without substantial continental boundaries to inhibit the flow, what prevents the current from accelerating indefinitely? While the circumpolar structure of the ACC resembles the atmospheric jet stream, which relies on horizontal momentum flux divergences to transfer momentum away from forcing regions [Gill, 1982], the systems are not completely analogous. Unlike the atmosphere, the ocean is subject to strong surface forcing from the wind in addition to topographic stresses at its bottom. The physical processes which remove the momentum input by the wind are the subject of this chapter. 
This study probes the dominant dynamical processes in the Southern Ocean, first by looking at the momentum balance in the quarter-degree version of the SemtnerChervin free surface primitive equation numerical model, and then by examining to what extent the results produced by the numerical model are consistent with altimeter estimates of mean sea surface height and variability in the Southern Ocean developed in chapters 2 and 3 . The momentum balance is considered in two coordinate systems, averaged zonally and along streamlines. In addition, in order to investigate the specific topographic features influencing the dynamics, this analysis will examine fluctuations in each of the terms in the momentum equations along streamlines. The results will show that the numerical model ACC undergoes substantial energy fluctuations along streamlines, but that the relative size of terms in the momentum balance does not change; therefore, eddy kinetic energy and Reynolds stress terms will be used to compare the model with altimeter data.

\subsection{Background: Processes Balancing Wind}

In theoretical and modeling studies, oceanographers have long speculated about the dominant forces controlling the momentum balance of the ACC. Consider the zonal momentum equation, in this case formulated in spherical coordinates as it is used in the Semtner-Chervin model [Semtner, 1986]:

$$
\begin{aligned}
\frac{\partial u}{\partial t}+\frac{1}{a \cos \phi} \frac{\partial(u u)}{\partial \lambda} & +\frac{1}{a \cos \phi} \frac{\partial(v u \cos \phi)}{\partial \phi}+\frac{\partial(w u)}{\partial z}-\frac{u v \tan \phi}{a}-f v= \\
& -\frac{1}{\rho_{0} a \cos \phi} \frac{\partial p}{\partial \lambda}+\mu \frac{\partial^{2} u}{\partial z^{2}}+A_{m} \nabla^{4} u
\end{aligned}
$$

where $u, v$, and $w$ represent zonal, meridional, and vertical velocity, $f$ is the Coriolis term, $\lambda$ and $\phi$ are longitude and latitude, $a$ is the radius of the Earth, $\rho_{o}$ is mean density, and $\mu$ represents a Richardson number dependent vertical viscosity. $A_{m}$, the horizontal viscosity, is defined as $-11 \times 10^{19} \cos ^{2}(\phi) \mathrm{cm}^{4} \mathrm{~s}^{-1}$. The dominant balance in 
(4.1) is between the Coriolis term, $f v$, and the pressure gradient term, $\frac{1}{\rho_{o} a \cos \phi} \frac{\partial p}{\partial \lambda}$. The tendency term, $\frac{\partial u}{\partial t}$, represents the change in momentum at a given location due to forcing, dissipation, and transport to other parts of the ocean. Momentum is input by the wind through the surface boundary condition for the vertical eddy viscosity term, $\mu \frac{\partial^{2} u}{\partial z^{2}}$. It is extracted via form drag, involving the pressure gradient term; bottom friction, represented as a bottom stress in the vertical viscosity term; and through no-slip lateral boundary conditions at continents and topography features. The other terms transfer momentum from where it enters at the surface to the boundaries where it is removed. Horizontal transfer occurs by biharmonic friction, $A_{m} \nabla^{4} u$ (often referred to as viscosity or momentum diffusion) and by horizontal momentum flux (or Reynolds stress) divergences, $\frac{1}{a \cos \phi} \frac{\partial(u u)}{\partial \lambda}$ and $\frac{1}{a \cos \phi} \frac{\partial(v u \cos \phi)}{\partial \phi}$. Vertical transfer results from vertical eddy viscosity and from the vertical momentum flux divergence, $\frac{\partial(w u)}{\partial z}$. The small metric term, $\frac{u v \tan \phi}{a}$, is an acceleration due to the curvature of the Earth,

In this study, we will consider the time averaged momentum equation:

$$
\begin{aligned}
& \frac{u_{f}-u_{i}}{\Delta t}+\frac{1}{a \cos \phi} \frac{\partial\left(\bar{u} \bar{u}+\overline{u^{\prime} u^{\prime}}\right)}{\partial \lambda}+\frac{1}{a \cos \phi} \frac{\partial\left(\bar{v} \bar{u} \cos \phi+\overline{v^{\prime} u^{\prime}} \cos \phi\right)}{\partial \phi} \\
& \quad+\frac{\partial(\bar{w} \bar{u})}{\partial z}+\frac{\partial \overline{w^{\prime} u^{\prime}}}{\partial z}-\frac{\overline{u v} \tan \phi}{a}-\frac{\overline{u^{\prime} v^{\prime}} \tan \phi}{a}-f \bar{v}= \\
& \quad-\frac{1}{\rho_{o} a \cos \phi} \frac{\partial \bar{p}}{\partial \lambda}+\mu \frac{\partial^{2} u}{\partial z^{2}}+A_{m} \nabla^{4} \bar{u}
\end{aligned}
$$

where overbars indicate time averages and primes indicate deviations from time averages. The terms $u_{f}$ and $u_{i}$ represent final and initial velocities and $\Delta t$ is the total time duration considered; these terms are used to estimate the velocity tendency. Time averaging the momentum equation minimizes the tendency term and allows us to compare the relative sizes of momentum flux divergences due to standing eddies, $\frac{1}{a \cos \phi} \frac{\partial(\bar{u} \bar{u})}{\partial \lambda}$ and $\frac{1}{a \cos \phi} \frac{\partial(\overline{v u} \cos \phi)}{\partial \phi}$, with those due to transient eddies, $\frac{1}{a \cos \phi} \frac{\partial \overline{u^{\prime} u^{\prime}}}{\partial \lambda}$ and $\frac{1}{a \cos \phi} \frac{\partial\left(\overline{v^{\prime} u^{\prime}} \cos \phi\right)}{\partial \phi}$. 
What physical processes counteract the substantial surface wind forcing in the Southern Ocean? In past studies, all of the terms in (4.2) have been considered in detail.

\subsubsection{Form Drag}

The leading candidate to balance wind stress is topographic form drag, associated with pressure gradients across bathymetric ridges, as proposed by Munk and Palmén [1951]. They provided a physically intuitive description illustrating how the pressure

force exerted on a topographic rise, produces a stress, $\frac{1}{\rho_{0}} \Delta p \Delta h$, where $\Delta p$ is the bottom pressure difference across the ridge, and $\Delta h$ the height of the ridge. The form drag is simply the pressure exerted on the deep zonal flow by a ridge.

Interpretation in terms of the meridional circulation is particularly illuminating. In the Southern Ocean, the mean eastward wind stress drives an Ekman flow northwards. A number of investigators have explored the implications of this ageostrophic surface transport [eg. Stommel, 1957; Toggweiler and Samuels, 1994; Marshall, 1994b]. Since no net flow out of the Southern Ocean is possible, this northward transport must be returned at deeper levels, where ocean processes are predominantly geostrophic, as illustrated in Figure 4.1. Geostrophic transport requires a tilting of isobaric surfaces, but in a continuous channel, density and pressure can undergo no net tilting between their starting point at $0^{\circ}$ and their return to the same point $360^{\circ}$ later. A net pressure gradient is possible only if the flow is intercepted by topography. The magnitude of the pressure difference between the upstream and downstream sides of a topographic obstruction provides a measure of the net isobaric tilting which may be achieved, and thus the net southward deep geostrophic flow which can counter the northward surface Ekman transport. While the geostrophic transport may be evenly distributed across an entire ocean basin or fully concentrated in an intense western boundary current, only the pressure difference across the ridge permits any net effect. Form drag is therefore simply a measure of the geostrophic 


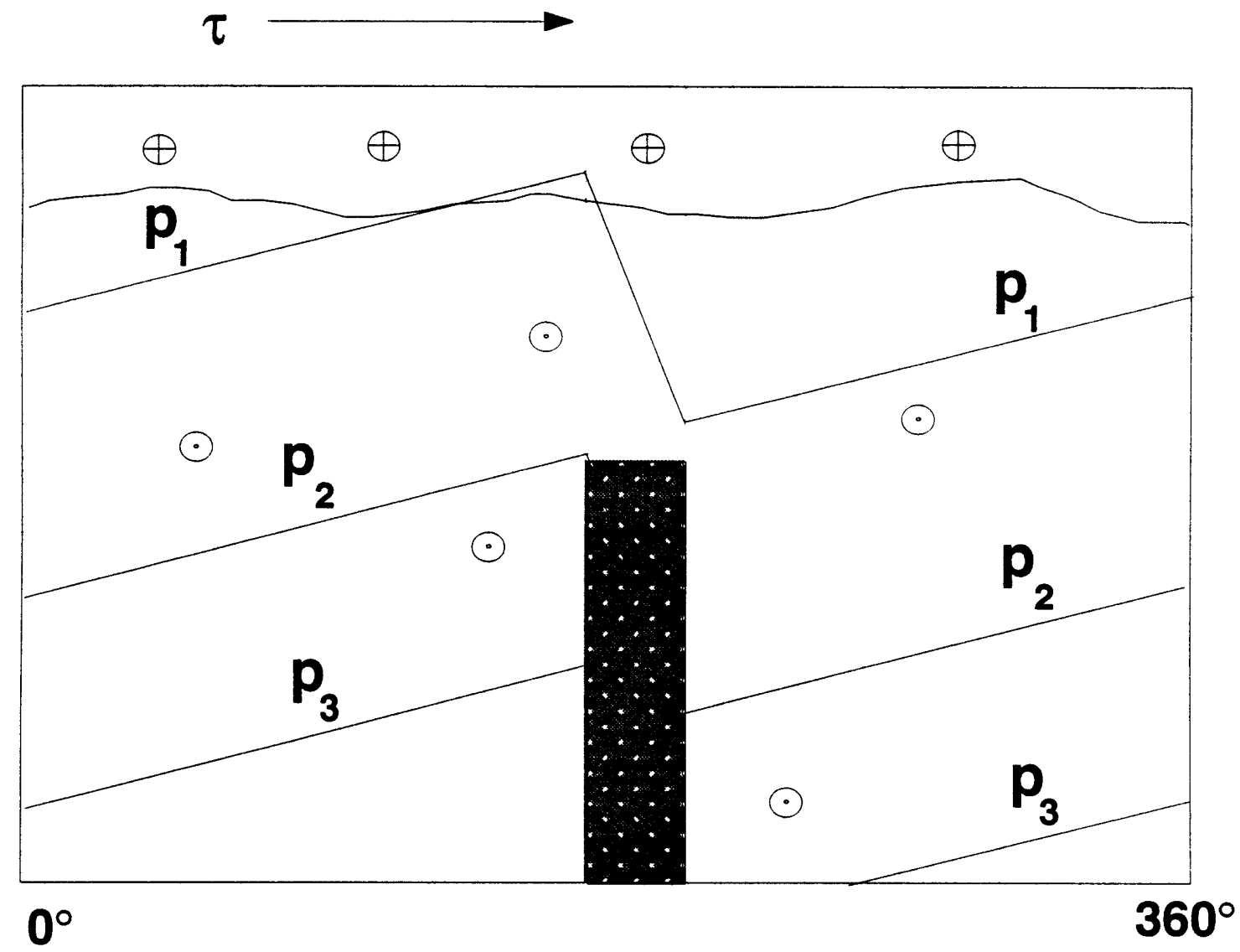

Figure 4.1: Diagram illustrating the role of form drag in the meridional circulation. Eastward wind stress $(\tau)$ drives a northward Ekman surface flow. Subsurface geostrophic flow returns water southward, as suggested by the isobars $\left(p_{i}\right)$. Since there can be no net geostrophic flow in an unblocked continuous channel (isobar $p_{1}$ ), only the geostrophic velocity below the level of the ridge results in a net geostrophic flow. If the flow is barotropic, as indicated in this diagram by parallel isobars, then northward geostrophic flow would be predicted directly over ridges. 
transport due to pressure differences across a topographic obstruction. Even in closed basins, form drag occurs as a result of the additional tilting of isobars made possible by the presence of a mid-basin ridge.

Mathematical descriptions of form drag are easily derived by zonally and vertically integrating the equations of motion [Johnson and Bryden, 1989]. For the time-averaged, steady-state case, there is no net transport into the Southern Ocean. Hence:

$$
-\oint \int_{-H}^{0} f \bar{v} d z d x=-\oint \int_{-H}^{0} \frac{1}{\rho_{o}} \frac{\partial \bar{p}}{\partial x} d z d x+\oint \frac{\overline{\tau^{x}}}{\rho_{o}} d x+\text { other terms }=0,
$$

where overbars represent time averages. Since $\frac{\partial p}{\partial x}$ integrates to zero at all unblocked depths, only the pressure differences across ridges and land masses will make any contribution to the net integrated term. Thus, the pressure gradient term on the right hand side may be interpreted as a summation of pressure differences across $N$ ridges:

$$
\oint \int_{-H}^{0} \frac{1}{\rho_{o}} \frac{\partial \bar{p}}{\partial x} d z d x=\frac{1}{\rho_{0}} \sum_{i=1}^{N} \int_{-H}^{0} \Delta \bar{p}_{i}(z) d z \approx \frac{1}{\rho_{o}} \sum_{i=1}^{N} \Delta \bar{p}_{i} \Delta z_{i} .
$$

where $\Delta p_{i}$ is the pressure difference and $\Delta z_{i}$ is the height of the $i$ th ridge. The term $\frac{1}{\rho_{0}} \Delta p_{i} \Delta z_{i}$ represents the form drag across the $i$ th ridge. Because form drag is equivalent to the vertical and zonal integral of the pressure gradient term, the term is most easily estimated by computing the quantity on the left of (4.4), rather than summing over the $N$ ridges.

Since there is no net meridional transport in the Southern Ocean, the meridional velocity, $v$, must vanish when integrated zonally and vertically. Therefore, (4.4) may be rewritten as:

$$
\begin{aligned}
-\oint \int_{-H}^{0} \frac{1}{\rho_{0}} \frac{\partial \bar{p}}{\partial x} d z d x & =\oint \int_{-H}^{0} f \bar{v} d z d x-\oint \int_{-H}^{0} \frac{1}{\rho_{0}} \frac{\partial \bar{p}}{\partial x} d z d x \\
& =\oint \int_{-H}^{0} f \bar{v} d z d x-\oint \int_{-H}^{0} f \bar{v}_{g} d z d x
\end{aligned}
$$

where $f v_{g}$, the geostrophic velocity multiplied by the Coriolis term, is the same as the pressure gradient term. Since the difference between total velocity, $v$, and geostrophic 
velocity $v_{g}$ is simply the ageostrophic velocity, $v_{a g}$, this expression shows that the total form drag represents the portion of the Coriolis term due to net ageostrophic velocity. That is:

$$
-\oint \int_{-H}^{0} \frac{1}{\rho_{0}} \frac{\partial \bar{p}}{\partial x} d z d x=\oint \int_{-H}^{0} f \bar{v}_{a g} d z d x
$$

Although in the circumpolar, vertically-integrated limit of (4.6), form drag is equivalent to the ageostrophic term, $f v_{a g}$, when a smaller sector of the ocean is considered, neither $\frac{\partial \bar{p}}{\partial x}$ nor the Coriolis term, $f \bar{v}$, integrates to zero. In such a case, the ageostrophic term encompasses other effects such as Ekman transport and eddy fluxes. If for example, only the upper ocean is considered, then no topographic barriers block the flow, and the pressure gradient integrates to zero; with no topography, the ageostrophic term cannot possibly represent topographic form drag, and is predominantly related to the surface Ekman transport. In contrast, within the portion of the ocean below the sill depth, all of the flow may be geostrophic, and there is no net ageostrophic flow that is readily identifiable as form drag.

The specific physical interpretation of the ageostrophic term, $f v_{a g}$, merits detailed consideration. Through a transformation of the continuity equation for an isopycnal layer, Marshall et al. [1993] argued that the ageostrophic velocity term is equal to the vertical divergence of the interfacial form drag averaged along geostrophic contours. Interfacial form drag, the drag exerted by one isopycnal layer on another, is entirely analogous to bottom form drag and is commonly invoked as the necessary mechanism to transfer momentum from the surface to the bottom. In a layered view of the ocean, horizontal flow exerts pressure on tilted isopycnal interfaces, thus transferring momentum from one layer to the next. If isopycnals are parallel (as suggested by Figure 4.1) then interfacial form drag is perfectly efficient, and all momentum which enters at the surface is transferred directly to the ocean bottom; otherwise, some momentum will move horizontally within the isopycnal layer. Therefore, Marshall et al.'s result indicates that momentum which is not transferred to the bottom 
via interfacial form drag is instead transferred laterally by the ageostrophic Coriolis term.

In a level model, flow along tilted isopycnals is equivalent to flow with a nonzero vertical velocity $w$. Thus perfectly efficient interfacial form drag corresponds to momentum transfer where isopycnals are parallel, and thus $w$ is constant with depth. In a non-rotating fluid, Gill [1982, chapter 8] showed that the divergence of interfacial form drag on a level surface $\left(\frac{\partial}{\partial z} \oint \frac{1}{\rho_{o}} p(d h / d x) d x\right)$ is equivalent to $\oint \frac{\partial(w u)}{\partial z} d x$. Rotation adds an additional effect due to the Coriolis term: To obtain a result analogous to Marshall et al.'s, the continuity equation for a level model (using Cartesian coordinates)

$$
\frac{\partial u}{\partial x}+\frac{\partial v}{\partial y}+\frac{\partial w}{\partial z}=0
$$

is integrated over an area bounded by the Antarctic continent in the south and by a circumpolar geostrophic streamline in the north. Multiplying by $f$ and integrating results in the expression:

$$
\oint f v_{a g} d x_{s}=-\int f \frac{\partial w}{\partial z} d A+\int \beta v d A
$$

where $v_{a g}$ represents the cross-stream ageostrophic velocity, $x_{s}$ is the along-stream coordinate, and $A$ is the total area considered. The $\beta v$ term is fairly small over this region, so that the cross-stream ageostrophic flow largely compensates the vertical flux through the entire volume. Thus the ageostrophic velocity term is an indication of the volume of water which must be brought southward to compensate upwelling necessary for surface Ekman transport. Equation (4.8) may be transformed to show an approximate link between the ageostrophic Coriolis term and vertical divergence of the interfacial form drag:

$$
\begin{aligned}
\oint f v_{a g} d x_{s} & =-\int f \frac{\partial w}{\partial z} d A+\int \beta v d A \\
& =-\frac{\partial}{\partial z} \int f \mathbf{u} \cdot \nabla h d A+\int \beta v d A \\
& =-\frac{\partial}{\partial z} \int \nabla \cdot(f \mathbf{u} h) d A+\frac{\partial}{\partial z} \int \beta v h d A+\int \beta v d A
\end{aligned}
$$




$$
=\frac{\partial}{\partial z} \oint f h \mathbf{k} \times \mathbf{u} d x_{\bullet}+\frac{\partial}{\partial z} \int \beta v h d A+\int \beta v d A
$$

where $h$ is the depth of an isopycnal surface. Substituting the momentum equation $((4.1)$ in Cartesian coordinates) into (4.9) and neglecting the minor correction terms due to $\beta$, which adjust for the irregularities in the boundary of the region considered, produces:

$$
\begin{aligned}
\oint f v_{a g} d x_{s} \approx & \frac{\partial}{\partial z} \oint\left[\frac{1}{\rho_{o}} h \frac{\partial p}{\partial x_{s}}+h \frac{\partial u u}{\partial x_{s}}+h \frac{\partial(w u)}{\partial z}-h \mu \frac{\partial^{2} u}{\partial z^{2}}-h A_{m} \nabla^{4} u\right] d x_{s} \\
= & -\frac{\partial}{\partial z}\left\{\oint \frac{1}{\rho_{o}} p \frac{\partial h}{\partial x_{s}} d x_{s}-\oint w u d x_{s}\right. \\
& \left.+\oint h\left[\frac{\partial(w u)}{\partial z}-\mu \frac{\partial^{2} u}{\partial z^{2}}-A_{m} \nabla^{4} u\right] d x_{s}\right\} .
\end{aligned}
$$

This in turn may be rewritten to show that the vertical divergence of interfacial form drag is:

$$
\frac{\partial}{\partial z} \oint \frac{1}{\rho_{0}} p \frac{\partial h}{\partial x_{s}} d x_{s}=-\oint f v_{a g} d x_{s}-\oint \frac{\partial w u}{\partial z} d x_{s}+\text { other terms }
$$

where the first term on the right is the ageostrophic term, the second is the nonrotational term identified by Gill, and the importance of the additional terms depends on the stratification and layer thickness. Using the geostrophic approximation to convert (4.9) to (4.10) according to quasigeostrophic scaling arguments would suppress the nonlinear terms which lead to Gill's expression for irrotational form drag, $\frac{\partial u w}{\partial z}$, and would more closely match Marshall et al.'s results. The inclusion of the nonlinear terms shows that the form drag is not exactly locally equivalent to the ageostrophic Coriolis term, $f v_{a g}$, but may be represented as a combination of this term plus the vertical eddy flux divergence, $\frac{\partial(w u)}{\partial z}$, plus additional correction terms.

While the divergence of the interfacial form drag is related to the ageostrophic Coriolis term, $f v_{a g}$, the interfacial form drag itself is associated with the meridional eddy density flux [Johnson and Bryden, 1989]. Expressing the isopycnal depth, $h$, in terms of the vertical density gradient, we write $h=\rho \frac{\partial z}{\partial \rho}$. Thus the form drag becomes:

$$
\oint \frac{1}{\rho_{o}} p \frac{\partial h}{\partial x_{s}} d x_{s} \approx \oint \frac{g f}{\rho_{o}} \frac{\overline{v_{g} \rho}}{N^{2}} d s
$$


where the Brunt-Väisälä frequency is defined as $N=\sqrt{-\frac{g}{\rho_{0}} \frac{d \rho_{0}}{d z}}$. The vertical transfer of momentum due to interfacial form drag is approximately equivalent to a meridional density flux, and is therefore tied to the meridional heat flux. The density flux will not be examined explicitly in this study.

The ageostrophic velocity term does not precisely represent form drag except in a vertically and circumpolarly integrated sense, and the meridional density flux is an approximation to interfacial form drag; rather than rely on either of these expressions, the form drag can be computed directly from the pressure gradient term in order to look at its regional influence. As discussed in the beginning of this subsection, form drag results from the increased tilting of isopycnals made possible by the presence of subsurface topography. In the circumpolar limit, no net tilt is possible, and the form drag is represented by the net geostrophic transport which actually occurs. Over a smaller region within the Southern Ocean, the isopycnals may have a net tilt which will imply a net geostrophic transport. Form drag is simply the difference between the geostrophic transport predicted from the pressure values on the boundaries of a region and the actual geostrophic transport. Thus the vertically integrated form drag is:

$$
\text { form drag }=\frac{1}{\rho_{0}} \int_{-H}^{0}\left(p_{f}-p_{i}\right) d z-\frac{1}{\rho_{0}} \int_{0}^{L} \int_{-H}^{0} \frac{\partial p}{\partial x} d z d x
$$

where $p_{i}$ is the westernmost pressure in the basin at a given latitude, $p_{f}$ is the easternmost pressure, and 0 and $L$ correspond to the western and eastern limits of the region. The pressure $p_{i}=p(0)$ and $p_{f}=p(L)$ except at levels below the ocean depth at $x=0$ or $x=L$. While this representation is useful for regional investigations, it is meaningless at a single point, and the ageostrophic term will also be used as a rough proxy for along-stream form drag to interpret the momentum balances in the Semtner-Chervin model in conjunction with the altimeter data. 


\subsubsection{Processes Considered in Past Studies}

In addition to form drag, a variety of other mechanisms have also been investigated to account for the Southern Ocean momentum balance: Gill [1968] considered a system where the wind stress is countered by bottom friction, but found that the frictional drag terms would need to be unrealistically large. As an alternative, he suggested that the wind stress could be balanced by a meridional momentum flux out of the ACC, to regions where continental boundaries would more readily remove the momentum. In an early numerical modeling effort, Gill and Bryan [1971] suggested that form stress might in fact accelerate the ACC. However, using a series of eddy resolving two-layer quasi-geostrophic numerical models, McWilliams et al. [1978] found that topographic form drag was an essential process limiting ACC transport. More recent numerical investigations have confirmed that the wind stress is balanced by form drag, rather than by frictional drag or momentum fluxes [Wolff et al., 1991; Marshall et al., 1993; Stevens and Ivchenko, 1994; Ivchenko et al. 1994; Killworth and Nanneh, 1994].

Although observations of the Southern Ocean are limited, data have generally supported the idea that the ACC momentum balance is controlled by form drag rather than horizontal momentum flux divergences or horizontal friction. Analyzing measurements from an array of current meters located southeast of New Zealand, Bryden and Heath [1985] found that the horizontal momentum flux divergence was too small to counteract the wind. Morrow et al. [1992] and Johnson et al. [1992] estimated the magnitude of the horizontal Reynolds stress terms from sea surface height variability measured by the Geosat altimeter data. Both altimetric studies concluded that the surface Reynolds stress fluxes were not statistically significant except in isolated locations such as the Agulhas retroflection region and the EltaninUdintsev Fracture Zone. In these locations the Reynolds stresses tended to be of the "wrong" sign, suggesting that the eddies drive the mean flow rather than dissipating it. By demonstrating that eddy processes are too weak to balance the Southern Ocean wind stress, these studies confirmed that form drag, involving a vertical rather than 
horizontal transfer of momentum, is the more likely mechanism balancing the wind stress.

Given the apparent importance of form drag, recent work has focused on understanding how momentum is transferred vertically through the water column to be dissipated on the ocean bottom. Johnson and Bryden [1989] suggested that the mesoscale eddy field might provide the mechanism needed to transport momentum downward through interfacial stress as represented in (4.12). They found order of magnitude agreement between interfacial form drag estimated from current meters in Drake Passage and the mean zonal wind stress. Drake Passage dynamics may not represent the entire ACC, however, so the circumpolar extrapolation of these results awaits confirmation. Analyses of numerical model results have specifically examined the roles of eddy fluxes and friction in transferring momentum horizontally and vertically within the Southern Ocean. In a two layer eddy-resolving quasi-geostrophic model, Wolff et al. [1991] found that momentum was transferred vertically through interfacial form drag set up mainly by the standing eddy field, while the transient eddies played only a minor role in the interfacial form drag and tended to accelerate the core of the flow. Reynolds stress divergences in the horizontal direction were considerably smaller, but showed a similar effect: divergences due to standing eddies tended to transport zonal momentum to latitudes where it is directly influenced by topography, while transient Reynolds stress terms acted to intensify the jet structure. In developing an analytic framework to interpret a three-layer quasi-geostrophic model, Marshall et al. [1993] argued that, despite their relatively smaller magnitude, transient eddies must flux properties across the ACC axis and thus play an essential role in determining the poleward heat flux and the vertical transfer of momentum. In contrast, because the cross-stream geostrophic velocity is zero, stationary geostrophic eddies should have no net effect on the ACC.

Results from a primitive equation general circulation model of the Southern Ocean, the Fine Resolution Antarctic Model (FRAM) [FRAM group, 1991], are gen- 
erally consistent with the earlier quasi-geostrophic analyses of ACC dynamics, both in showing the overall importance of bottom form drag for the vertically averaged momentum balance and in demonstrating the vertical transfer of wind stress through the action of interfacial form drag. Stevens and Ivchenko [1994] considered the zonal momentum balance of the ACC for the latitudes which are unblocked by Drake Passage. At the upper level of the model, surface wind stress is balanced by meridional Ekman flux; at intermediate depths, the poleward Reynolds stress divergence balances the ageostrophic component of velocity, and at deeper levels where bottom obstructions appear, the Coriolis term is balanced by the momentum flux divergence and the pressure gradient terms. By linking the interfacial form stress to the meridional density flux, Stevens and Ivchenko demonstrated that interfacial form drag is able to transfer momentum vertically. In order to minimize the effects of standing eddies which drive the mean ACC north and south, but have no net impact on the momentum balance on any given streamline, Ivchenko et al. [1994] followed the suggestion of Marshall et al. [1993] to consider the momentum balance in stream coordinates. While form drag remains the dominant stress balancing the wind, in place of a strong mean momentum flux divergence in the vertically averaged balance, they found that lateral friction was the next largest term, and was typically 30 to $50 \%$ of the magnitude of form drag. The mean vertical momentum flux divergence and the transient eddy flux divergence contributed smaller adjustments to the net balance. Analyses also showed that the buoyancy flux, stratification, and isopycnal outcropping all contribute to the penetration of surface stress via interfacial form drag. Killworth and Nanneh [1994] examined the output of the FRAM model in isopycnal coordinates, which permitted a more direct analysis of interfacial form drag. They found that on any given isopycnal layer in the interior, the dominant balance was between interfacial form drag on the upper surface of the layer and interfacial form drag at the bottom of the layer; in other words, the parallel isopycnal surfaces defining the top and bottom of a layer each transfer momentum downward through the water column so that there is no 
net gain within the layer, but momentum is transported to depths where topographic form drag may act. On each isopycnal surface, net interfacial form drag was balanced by the Coriolis term and, in the layers which outcrop, by wind stress. Since form drag is represented by the pressure gradient term, in essence, their results amount to an assertion that the system is predominantly geostrophic along isopycnal surfaces with an ageostrophic Ekman surface transport balancing the wind stress. They did not look beyond this first order balance to consider the vertical divergence of interfacial form drag and the specific role of eddy processes in transferring momentum meridionally.

Other, more process oriented studies have probed the individual physical effects at work and their role in determining total ACC transport. A primitive equation model of the Atlantic-Indian sector of the Southern Ocean [Olbers et al., 1992] and a quasi-geostrophic one-layer model driven with observed pressure forcing [Klinck, 1992] have indicated that in addition to the wind stress, vertical stratification plays a crucial role in determining the net transport of the ACC. The specific character of the topography also appears to be significant. Treguier and McWilliams [1990], using a quasi-geostrophic model, found that wider ridges were particularly important in controlling the ACC, while smaller features had much less impact on the overall momentum balance. In an analytic study, Wang [1993] showed that for channel flow on a $\beta$-plane, the $\beta$-effect tends to steer flow over ridges; only topographic features exceeding a critical height scale provide any drag. Both Wang's and Treguier and McWilliams' conclusions imply that the effects of topography might be confined to a few isolated features within the Southern Ocean.

Together these recent results describe an evolving picture of the wind-driven circulation of the Southern Ocean, in which meridional and vertical eddy fluxes intensify the jet structure and interfacial form drag, represented by a meridional density flux, transfers momentum downwards to be dissipated through bottom form drag. Still it remains unclear how representative model dynamics are of Southern Ocean 
physics. In one of few detailed comparisons, Wilkin and Morrow [1994] examined the Reynolds stress analysis of Geosat data together with the Southern Ocean eddy processes in the half-degree version of the Semtner-Chervin model. Though they found substantial quantitative differences in eddy kinetic energy, in the high energy Agulhas region, eddy-mean flow interaction terms were in qualitative agreement for the data and model. In this study output from a higher resolution version of the Semtner-Chervin model will be examined.

\subsection{Momentum Information from Model Output}

The Semtner-Chervin model is a 20-level global general circulation model based on the Cox-Bryan code [Semtner, 1986]. It solves the zonal primitive equation (4.1) and the analogous meridional expression. Although the specifics of the resolution and forcing differ, the basic model formulation is the same as FRAM [FRAM group, 1991], and many of the results are qualitatively similar. For this study results were analyzed from the "quarter-degree" version, which implemented several refinements from the earlier half-degree version [Semtner and Chervin, 1992]. The horizontal resolution is $0.4^{\circ}$ in the zonal direction, and $0.4^{\circ} \cos (\phi)$ in the meridional direction, so that in this version square grid boxes are maintained, and the horizontal resolution increases at higher latitudes. This resolves the first baroclinic Rossby radius: between $70^{\circ} \mathrm{S}$ and $30^{\circ} \mathrm{S}$ the model resolution ranges from 15 to $38 \mathrm{~km}$, while the Rossby radius, estimated using the Raleigh-Ritz method discussed in section 2.5.1, varies from 40 to $160 \mathrm{~km}$.

In the quarter-degree model a free sea surface was implemented based on the formulation by Killworth et al. [1991]. The model was initialized with the output from the half-degree model following 22.5 years of spin-up and 10 years of forcing with Hellerman and Rosenstein winds. During the last 5 years of the half-degree run, 
all deep relaxation to Levitus climatology was eliminated [Semtner and Chervin, 1992; Semtner, personal communication]. At one-quarter degree resolution, the model was then run for one year using climatological winds. After spin-up, it was forced with ECMWF operational forecast model winds [Trenberth et al., 1989] for the period from January 1986 to December 1989. Surface buoyancy forcing was based on the Levitus monthly climatologies with a one-month relaxation time scale. In addition, temperature and salinity were relaxed to "annual mean Levitus" climatology in the upper $2000 \mathrm{~m}$, within $7^{\circ}$ of the northern and southern boundaries of the model domain and within $7^{\circ}$ of the Straits of Gibraltar with a three month time scale. Unlike older versions of the model, this version included no relaxation to Levitus climatology below $2000 \mathrm{~m}$. The results for $3-\frac{3}{4}$ years, starting with April 1986, were time-averaged to produce mean model statistics.

The model is formulated on the Arakawa B-grid: temperature-salinity points are located at the center of model grid boxes; velocity points are on the corners in the horizontal direction at the same depth as temperature and salinity points. Thus geostrophic velocities are computed using simple two-point differences of the computed pressure terms.

Boundary conditions are:

$$
\mu \frac{\partial u}{\partial z}=\frac{\tau^{\lambda}}{\rho_{o}}
$$

at the surface, and

$$
\mu \frac{\partial u}{\partial z}=b u \sqrt{u^{2}+v^{2}}
$$

at the bottom, where $b$, the bottom friction coefficient, is unitless and is defined as $1.225 \times 10^{-3}$. No slip boundary conditions are imposed on the side walls, so that $u$ and $v$ are zero at all grid points corresponding to land for the horizontal gradient terms.

While investigations of the mean momentum balance in FRAM [Stevens and Ivchenko, 1994; Ivchenko et al., 1994; Killworth and Nanneh, 1994] have averaged 72 instantaneous monthly model dumps, the Semtner-Chervin model output includes 
continuously time-averaged means, variances, and covariances of the model variables. The impact of the nonlinear advective terms in the momentum balance may be examined by computing derivatives of the stored $u, v$, and $w$ variance and covariance terms. This permits a reasonable approximation of all of the terms in the momentum balance.

Several subtleties in the model formulation mean that perfect estimates of these mean terms cannot be obtained so that the momentum balance will not close completely. Although the term $f \bar{v}$ may be determined exactly, the pressure gradient term, $\frac{1}{\rho_{0} a \cos \phi} \frac{\partial \bar{p}}{\partial \lambda}$, is not stored by the numerical model and must be calculated by applying the model equation of state to the mean temperature and salinity values. A partial correction for the nonlinearities in the equation of state is made using the standard deviations of temperature and salinity (see the appendix) but cannot be expected to account for all of the nonlinearities. The calculation is quite sensitive to numerical errors and must be done in double precision. The vertical viscosity, $\mu$, defined by Pacanowski and Philander [1981], is also time-dependent and highly nonlinear. In the deep ocean, $\mu$ is small and roughly constant at $1 \mathrm{~cm}^{2} \mathrm{~s}^{-1}$, but at shallower depths, nonlinearities in $\mu$ may cause significant errors in the momentum balance. Since $\mu$ depends on covariances of vertical derivatives, which are not stored, it may not be corrected using the variance formulation outlined in the appendix for the pressure gradient term. Thus the time mean of upper ocean vertical viscosities cannot be calculated directly. In addition, errors may have been incurred in recomputing the wind field used for the Semtner-Chervin runs. Thus, surface values of the vertical viscosity term will be inferred from the residual differences in the momentum balance. Convective adjustment and surface relaxation to Levitus are additional processes in the model, but they will not be considered here as they are minute factors in the momentum balance, acting only indirectly by changing temperature and salinity values slightly between adjacent time steps. 
Additional errors arise from several of the other terms in the momentum equations. In the model formulation, the nonlinear advective terms are computed by adding adjacent values and then multiplying and computing derivatives, so that the time averaged results involve not only the correlations $\overline{u^{\prime} u^{\prime}}, \overline{v^{\prime} v^{\prime}}$ and $\overline{u^{\prime} v^{\prime}}$ which are stored in the mean model output, but also the correlations of adjacent points (for example, $\left.\overline{u_{j, i+1}^{\prime} v_{j, i}^{\prime}}\right)$ which are non-negligible, and contribute to the misfit in the final results.

The vertical advective terms also play a significant role in the momentum balance. The vertical velocity $w$ is computed at the same locations as $u$ and $v$ for dynamical computations, and at the same locations as $T$ and $S$ for tracer computations, but only the tracer computation value is stored. Differences between these values can be substantial, so in this analysis $\bar{w}$ is recomputed directly from $u$ and $v$; however the stored covariance terms $\overline{u^{\prime} w^{\prime}}$ and $\overline{v^{\prime} w^{\prime}}$ rely on $w$ at $T, S$ points, so that the dynamical vertical advection terms cannot be determined exactly. Nonetheless, errors induced by these minor technical problems do not significantly affect the major terms in the momentum balance.

In the Southern Ocean, the model indicates the flow to be confined to two or more narrow jets, in general agreement with hydrographic observations [Orsi, 1993] and altimeter measurements (see chapter 2). The average transport through Drake Passage in the model output is $200 \times 10^{6} \mathrm{~m}^{3} \mathrm{~s}^{-1}$, which exceeds the observed value of $130 \times 10^{6} \mathrm{~m}^{3} \mathrm{~s}^{-1}$ by about $50 \%$. Although the transports are of similar magnitudes, the large discrepancy suggests that the model may not fully capture the physical conditions governing the ACC. The next sections will examine the critical dynamics in the model momentum balance. 


\subsection{Vertically Integrated Momentum Balance}

\subsubsection{A Meridional Slice}

A single vertically and temporally integrated meridional slice offers a measure of the leading physical processes controlling the entire water column. Figure 4.2 shows that geostrophy provides the dominant balance in the momentum equations at $24.2^{\circ} \mathrm{E}$, as elsewhere in the model. The difference between the pressure gradient term and the Coriolis term is less than $10 \%$ of their respective values. Since the geostrophic balance is nearly exact, and the difference between the geostrophic and total velocities provides the critical form drag component in the momentum balance, small errors in each term may lead to substantial errors in the momentum balance.

\subsubsection{Zonally Averaged Balance}

Zonally averaging and vertically integrating the momentum equations allows us to consider the momentum balance for the full Southern Ocean. Since no net transport enters the system, the Coriolis term, $f \bar{v}$, integrates to zero, and the zonally integrated pressure gradient term is equivalent to $-f \bar{v}_{a g}$ and represents form drag, as discussed in section 4.2.1. This form drag balances wind stress, as shown in Figure 4.3. The balance agrees with findings from the FRAM model [Stevens and Ivchenko, 1994; Killworth and Nanneh 1994]. However, while previous studies have focused largely on the zonally unblocked region between $55^{\circ} \mathrm{S}$ and $62^{\circ} \mathrm{S}$, in this analysis latitudes intersected by continents are included; the presence of subsurface topography at even the unblocked latitudes means that the pressure gradient term does not vary dramatically between blocked and unblocked latitudes.

Bottom friction is negligibly small, so that the residual difference between the form drag and the wind stress is an indication of the amount of momentum which is transferred meridionally rather than vertically. This meridional transfer of momentum 

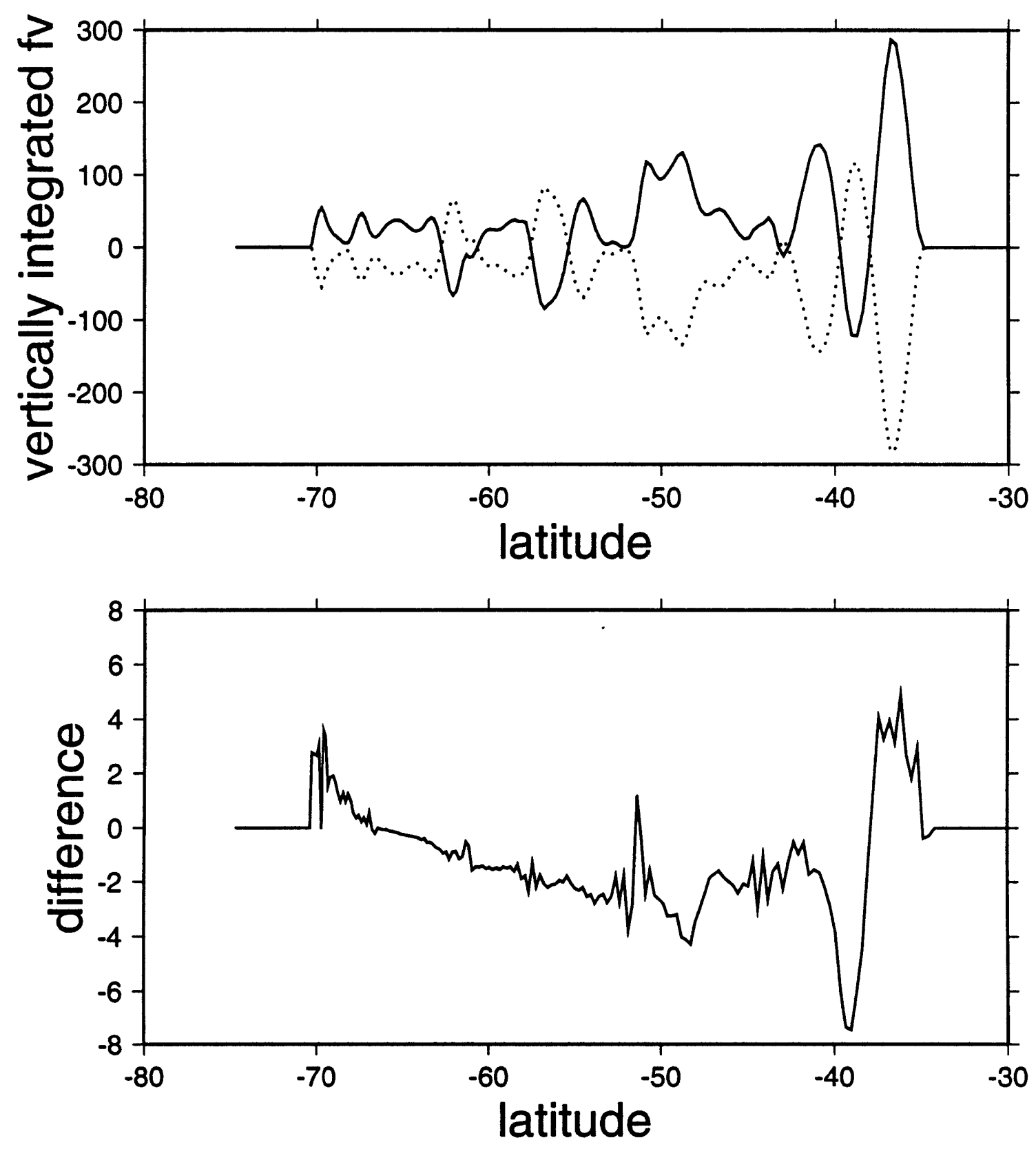

Figure 4.2: Vertically integrated geostrophic balance in the quarter-degree SemtnerChervin model output in $\mathrm{cm}^{2} \mathrm{~s}^{-2}$, at $24.2^{\circ} \mathrm{E}$. Upper panel shows (solid line) the Coriolis term, $\int f \bar{v} d z$, and (dotted line) the pressure gradient term, $-\int f \bar{v}_{g} d z=$ $-\int \frac{1}{\rho_{0}} \frac{\partial \bar{p}}{\partial x} d z$. The lower panel shows the difference between the two, $\int f \bar{v}-f \bar{v}_{g} d z=$ $\int f \frac{p_{0}}{\bar{v}_{a g}} d z$, the ageostrophic term, which is due to a combination of form drag, wind stress, and nonlinear advective terms. 

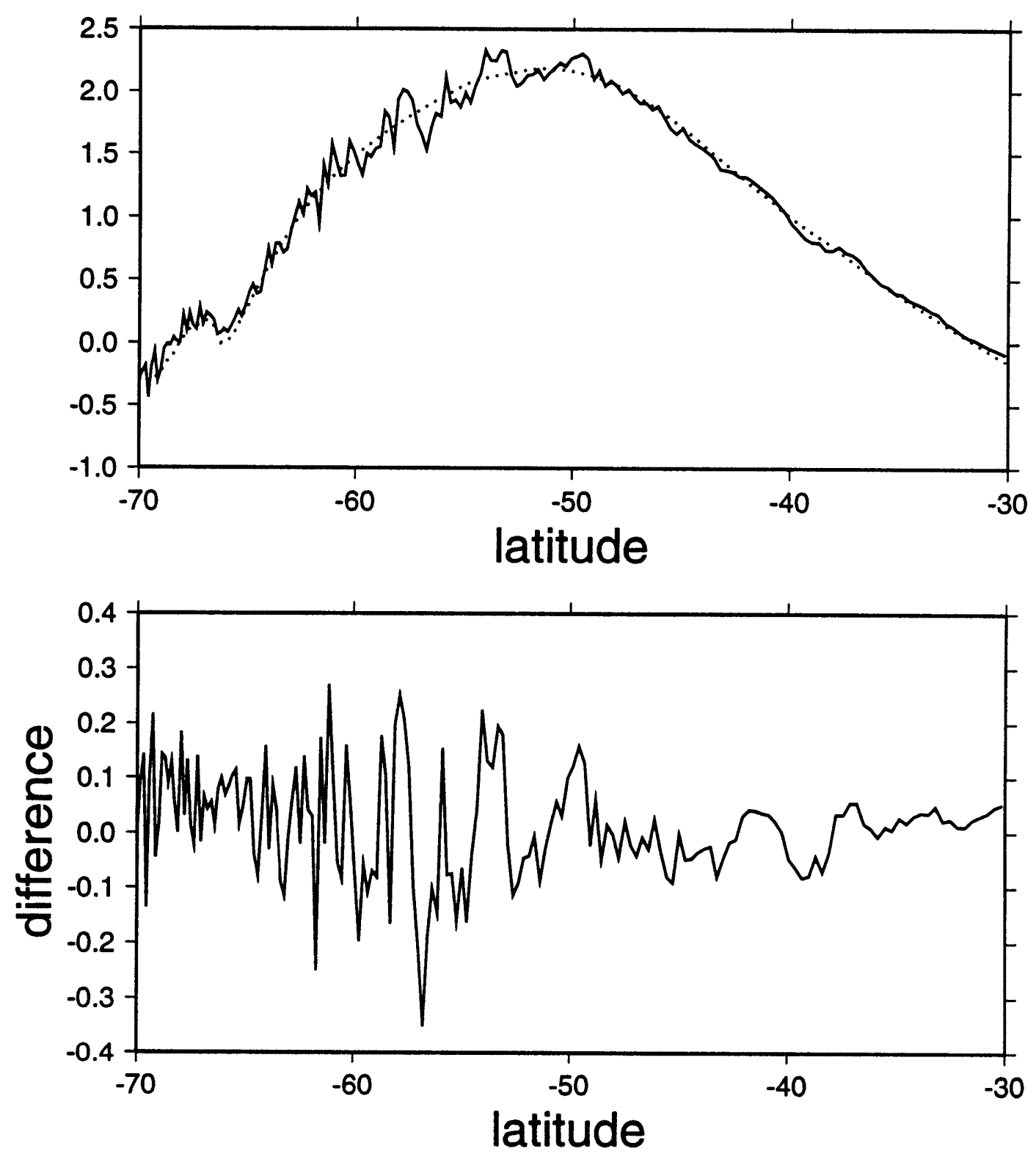

Figure 4.3: Vertically integrated and zonally averaged dominant momentum balance in the quarter-degree Semtner-Chervin model output in $\mathrm{cm}^{2} \mathrm{~s}^{-2}$. Upper panel shows (solid line) the pressure gradient term, $\frac{1}{L} \iint \frac{-1}{p_{0}} \frac{\partial p}{\partial x} d x d z$, which is the form drag, and (dotted line) the integrated stress term $\frac{1}{L} \iint \mu \frac{d^{2} u}{d z^{2}} d x d z \approx \frac{1}{L} \int \frac{\tau^{*}}{\rho_{0}} d x$. Lower panel shows the difference between the form drag and wind stress. 
and the corresponding transfer of heat and salinity play a critical role in linking the Southern Ocean with the closed ocean basins to its north. The contribution of each of the terms in (4.2) to this residual momentum balance is considered. The nonnegligible terms, plotted in Figure 4.4 are the meridional mean momentum divergence, $\frac{\partial \bar{u} \bar{v}}{\partial y}$, the biharmonic friction term, $A_{m} \nabla^{4} \bar{u}$, and the meridional eddy momentum divergence, $\frac{\partial \overline{u^{\prime} v^{\prime}}}{\partial y}$. (Here, and in the remainder of the momentum balance discussion, Cartesian coordinates $(x, y)$ are used for simplicity.) The most significant contribution to the cross-stream momentum balance is due to the mean eddy flux divergence (line $\mathrm{b}$ in Figure 4.4) and is noticeably larger than the eddy flux divergence from transient eddies (line c). The overall results are consistent with the findings of Stevens and Ivchenko [1994] for the FRAM model. In the Semtner-Chervin model, biharmonic friction (line d) appears more substantial than FRAM results would indicate, but this effect is most noticeable at high latitudes, outside of the FRAM analysis region, where friction removes point to point spikiness. The remaining terms from equation (4.2), related to the vertical advection of momentum, bottom friction, and longterm velocity tendency, are too small to explain the residual momentum imbalance (indicated by line e in Figure 4.4); instead, the residual is probably due to small errors in the wind field used for this analysis, nonlinearities in the pressure gradient term (see the appendix to this chapter), nonlinearities in the surface Richardson number dependent vertical viscosities, and errors in computing the meridional eddy flux divergence terms.

\subsubsection{Average Balance Along Streamlines.}

Marshall et al. [1993] argued that standing eddies have no net impact on the momentum balance. Properties fluxed northward in one large stationary meander of the ACC, will return southward within the ACC, providing no net flux of momentum or other properties along the circumpolar path. To eliminate the effect of standing eddies, they advocated studying the momentum balance in stream coordinates; the 


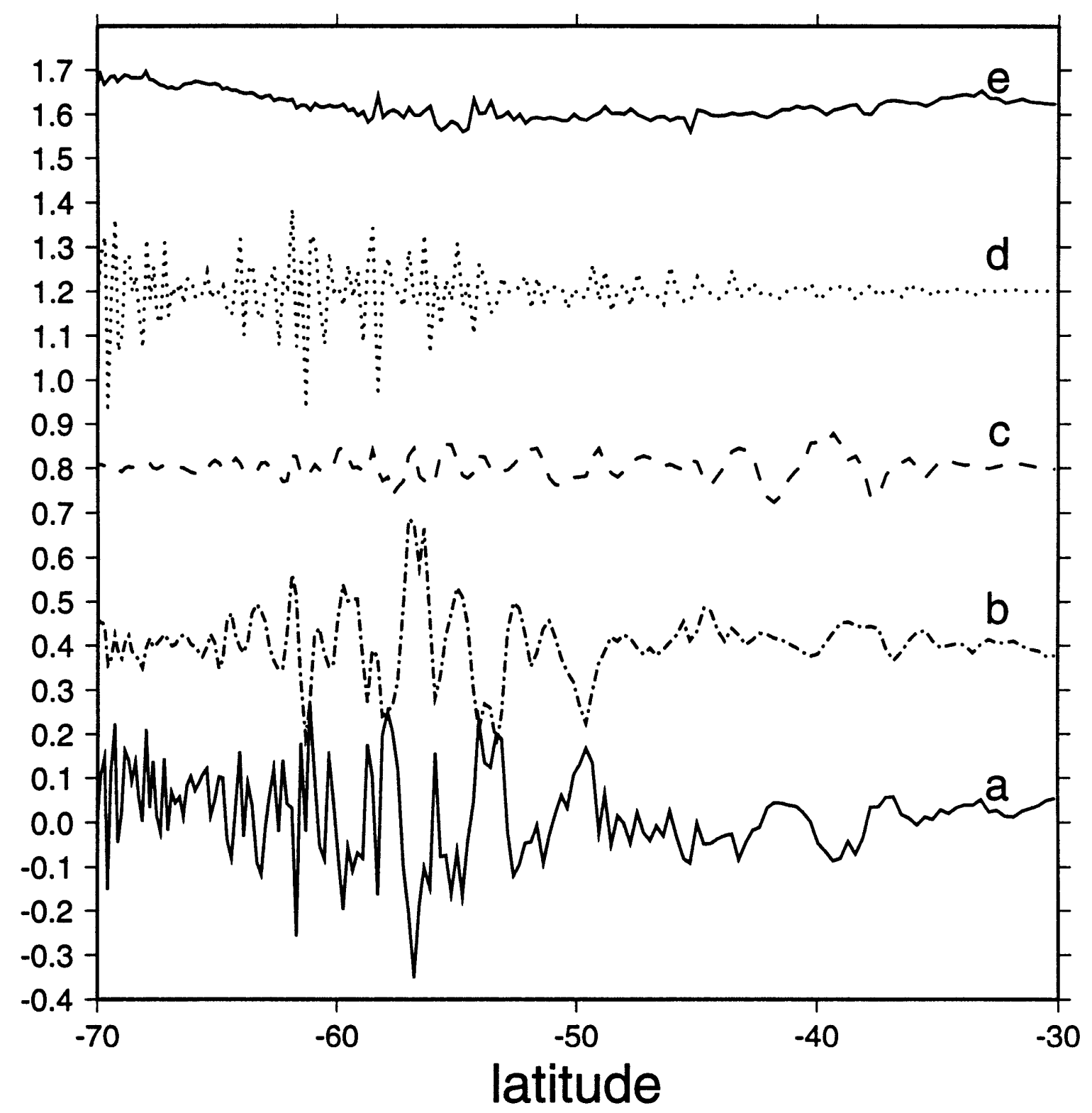

Figure 4.4: Vertically integrated and zonally averaged momentum balance in the quarter-degree Semtner-Chervin model output in $\mathrm{cm}^{2} \mathrm{~s}^{-2}$. Terms shown are (a) the component of form drag not balanced by wind stress $\left(\frac{1}{L} \iint \frac{-1}{\rho_{0}} \frac{\partial \bar{p}}{\partial x}+\mu \frac{d^{2} \bar{u}}{d z^{2}} d x d z\right)$, (b) the mean equatorward momentum flux divergence $\left(\frac{1}{L} \iint \frac{\partial \overline{u v}}{\partial y} d x d z\right)$, (c) the equatorward eddy flux divergence $\left(\frac{1}{L} \iint \frac{\partial \overline{u^{\prime} v^{\prime}}}{\partial y} d x d z\right.$ ), and (d) the biharmonic friction term $\left(\frac{1}{L} \iint A_{m} \nabla^{4} \bar{u} d x d z\right)$. Term (e) represents the residual imbalance. The offset between successive terms is $0.4 \mathrm{~cm}^{2} \mathrm{~s}^{-2}$. 
geostrophic component of the mean cross-stream Coriolis term $\left(f v_{g}\right)$ is zero, and only ageostrophic effects, transient eddies, and frictional effects may act to transport momentum across the ACC.

For vertically averaged flow, there is no easily defined geostrophic contour onto which the equations of motion may be translated. Instead, consider the flow along the sea surface height contours, $\eta$, defined by the model free surface, as shown in Figure 4.5. The equations of motion are translated into stream coordinates by first defining continuous height contours, then determining the zonal distance, $s_{x}$, and meridional distance, $s_{y}$, between consecutive points, and finally rotating the equations, using a cosine/sine transformation, so that:

$$
\text { along-stream momentum }=M_{x} \times \frac{s_{x}}{\sqrt{s_{x}^{2}+s_{y}^{2}}}+M_{y} \times \frac{s_{y}}{\sqrt{s_{x}^{2}+s_{y}^{2}}}
$$

where $M_{x}$ is the $x$-momentum equation and $M_{y}$ the $y$-momentum equation. Note that curvature terms are not introduced into this expression for along-stream momentum, because the advective and frictional terms which would be altered by the change in coordinates are computed before converting to along-stream coordinates. ${ }^{1}$

${ }^{1}$ The full expression for along-stream momentum in stream coordinates is:

$$
\begin{aligned}
& \frac{u_{s f}-u_{s i}}{\Delta t}+\frac{1}{r} \frac{\partial\left(\bar{u}_{s} \bar{u}_{s}+\overline{u_{s}^{\prime} u_{s}^{\prime}}\right)}{\partial \theta}+\frac{\partial\left(\bar{v}_{s} \bar{u}_{s}+\overline{v_{s}^{\prime} u_{s}^{\prime}}\right)}{\partial r}+\frac{\overline{u_{s} v_{s}}}{r}+\frac{\overline{u_{s}^{\prime} v_{s}^{\prime}}}{r} \\
& \quad+\frac{\partial\left(\bar{w} \bar{u}_{s}\right)}{\partial z}+\frac{\partial \overline{w^{\prime} u_{s}^{\prime}}}{\partial z}-f \bar{v}_{s}=-\frac{1}{r} \frac{\partial \bar{p}}{\partial \theta}+\mu \frac{\partial^{2} u_{s}}{\partial z^{2}} \\
& +A_{m}\left[\nabla^{4} \bar{u}_{s}-\frac{2 \nabla^{2} \bar{u}_{s}}{r^{2}}-\frac{4}{r^{2}} \frac{\partial}{\partial \theta}\left(\nabla^{2} \bar{v}_{s}\right)-\frac{4}{r^{4}} \frac{\partial^{2} \bar{u}_{s}}{\partial \theta^{2}}-\frac{2}{r^{4}} \frac{\partial \bar{u}_{s}}{\partial \theta}-\frac{6}{r^{4}} \frac{\partial \bar{v}_{s}}{\partial \theta}-\frac{3 \bar{u}_{s}}{r^{4}}\right]
\end{aligned}
$$

where $u_{s}$ is the along-stream velocity, $v_{s}$ is the cross-stream velocity, $r$ represents the radial (or $v_{s}$ ) direction, and $\theta$ represents the along-stream direction. In converting the momentum equation from spherical coordinates to stream coordinates, the metric terms have been omitted since they are extremely small. The term $\frac{u_{e} v_{n}}{r}$ is the acceleration due to curvature of the streamlines in the new coordinate system. The cross-stream velocity, $v_{A}$, is extremely small in stream coordinates, so that only the transient component of the curvature term need be retained. Assuming that the model fully resolves mesoscale features, so that $r$ is much larger than the model resolution, the curvature term should be much smaller than $\frac{\partial u_{r}, v_{e}}{\partial r}$. Converting to stream-coordinates also substantially complicates the biharmonic friction term, but all components containing $v_{\text {s }}$ are negligible, and in fact, if variations in the cross-stream direction are substantially larger than the radius of curvature $r$ or variations in the along-stream direction, then the largest of the additional terms should be $-\frac{2 \nabla^{2} \bar{u}_{2}}{r^{2}}$. This term, 


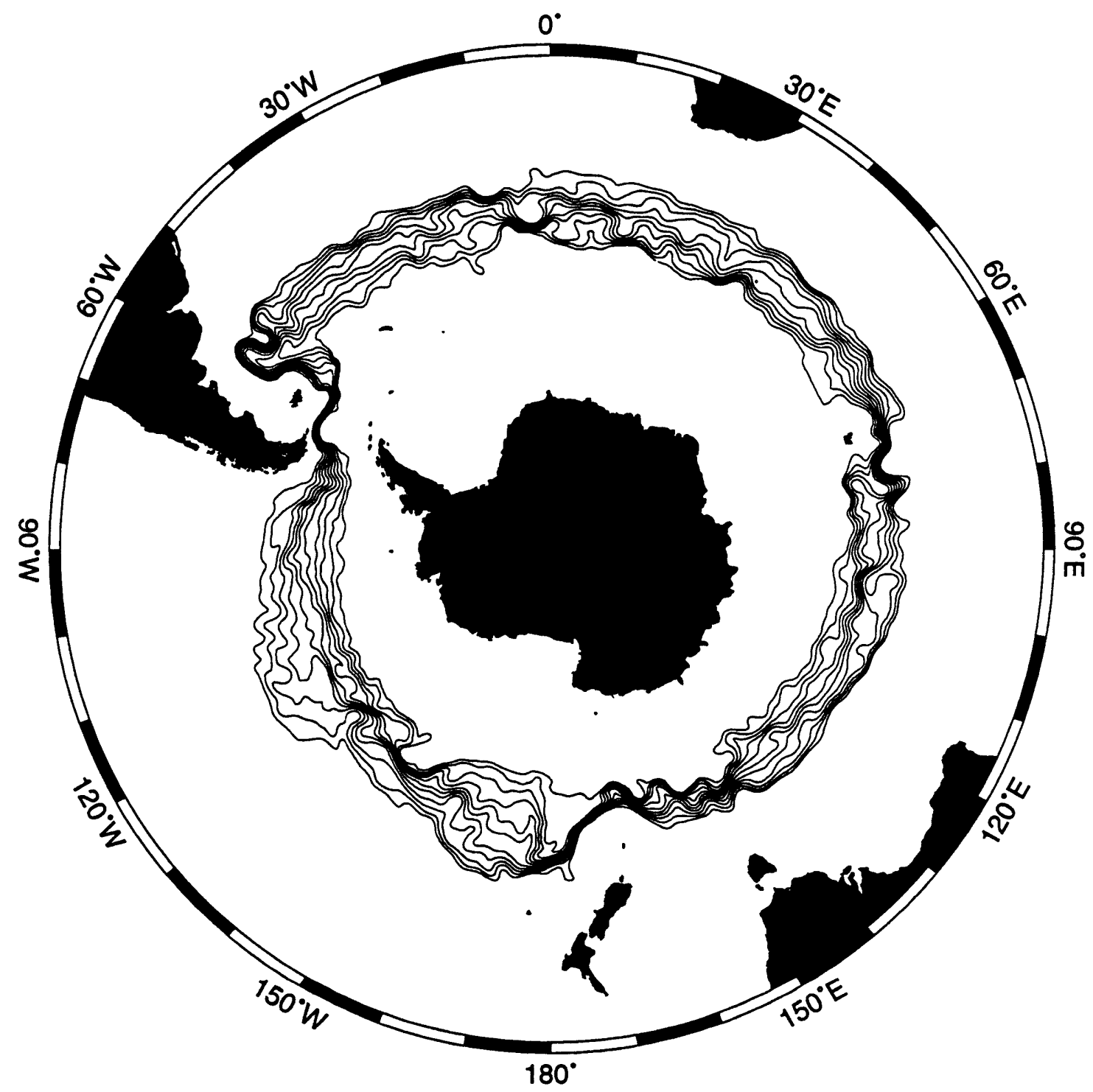

Figure 4.5: Sea surface height contours used for mapping vertically integrated momentum balance in stream coordinates. Contours extend from $-0.5 \mathrm{~m}$ at the south to $0.5 \mathrm{~m}$ at the north, with a contour interval of $0.1 \mathrm{~m}$. 
The resulting equation is essentially identical to (4.2) except that all coordinates are rotated into stream-coordinates.

The results shown in Figure 4.6 are analogous to the zonally averaged results: the total transport across streamlines integrates to zero; the pressure gradient term, integrated along streamlines, represents the form drag. This form drag provides the dominant balance for wind stress, just as in the zonally averaged case, as indicated in the upper panel of Figure 4.6. The residual difference between wind stress and form drag is substantially different from the zonally averaged case, as shown in the middle panel of Figure 4.6. While mean eddies dominated the zonally averaged balance, in the along-stream average, substantial contributions come from a combination of biharmonic friction, and transient and mean eddy flux divergences. Biharmonic friction is the largest of these terms, as Ivchenko et al. [1994] found for FRAM; this suggests that viscous dissipation, rather than eddy flux, is the critical mechanism transporting properties across the ACC in both the FRAM and Semtner-Chervin formulations of this numerical model, in opposition to Marshall et al.'s hypothesis. If the biharmonic friction term is representative of ocean physics, this suggests that subgrid scale processes are more important than resolved eddy processes in fluxing momentum across the ACC. Thus the model may not adequately resolve the eddy anisotropies necessary to represent cross-stream momentum transfer.

In addition, the mean eddy flux term $\left(\frac{\partial \overline{u, v a}}{\partial y}\right)$ is larger than the transient eddy flux term $\left(\frac{\partial \bar{u}^{\prime}, v_{t}^{\prime}}{\partial y}\right)$, seeming to suggest that stationary eddies are more significant than transient eddies in fluxing momentum across the ACC. This effect can be attributed to several factors, some of which are misleading. First, the largest contribution occurs because the surface streamlines used for this analysis are not precisely parallel to the deep geostrophic flow, so we can predict a finite deep cross-stream geostrophic component to the mean eddy flux term. Second, a large Ekman transport at the surface

in turn, is $\frac{L^{2}}{r^{2}}$ smaller than the biharmonic term, where $L$ is the scale of cross-stream variation in velocity. 


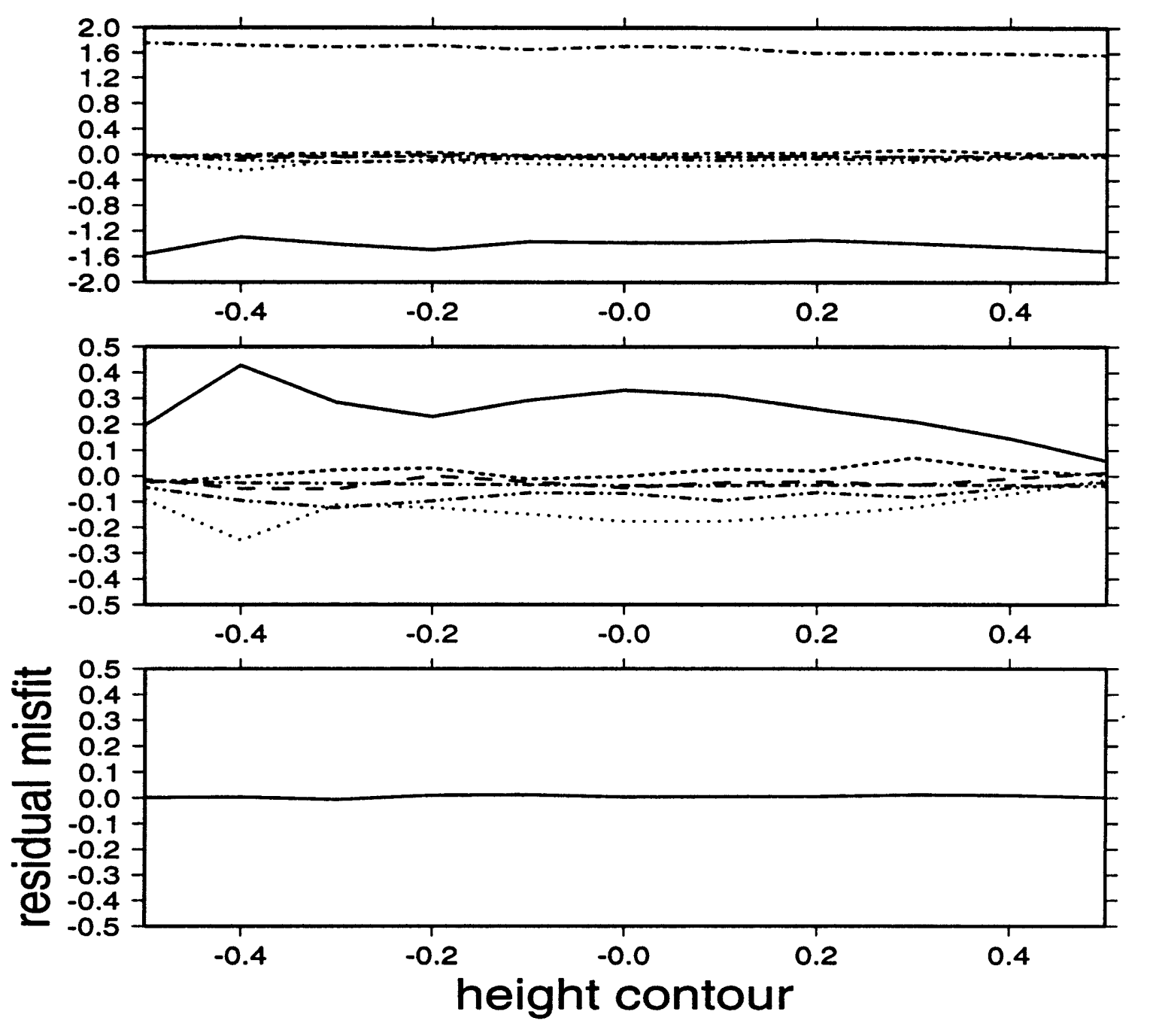

Figure 4.6: Momentum balance integrated vertically and averaged along stream lines in the quarter-degree Semtner-Chervin model output in $\mathrm{cm}^{2} \mathrm{~s}^{-2}$. Upper panel shows (solid line) the ageostrophic velocity $\left(\frac{1}{L} \iint f v-f v_{g} d s d z\right.$ ) rotated into stream coordinates, (dash-dot line) the mean wind stress, (dash double-dotted line) the mean equatorward momentum flux divergence $\left(\frac{1}{L} \iint \frac{\partial \overline{u v}}{\partial y} d s d z\right.$ ), (long dashed line) the transient equatorward eddy flux divergence $\left(\frac{1}{L} \iint \frac{\partial \overline{u^{\prime} v^{\prime}}}{\partial y} d s d z\right.$ ), (dotted line) the biharmonic friction term $\left(\frac{1}{L} \iint A_{m} \nabla^{4} \bar{u} d s d z\right.$ ), (short dashed line) mean vertical flux divergence, and (double dashed double-dotted line) bottom friction. The middle panel shows the same information, except that the solid line represents the portion of the ageostrophic velocity not accounted for by the wind stress. The lower panel shows the residual momentum imbalance when all these terms are summed. If the momentum balance were perfect, this term would be constant at zero. The errors inherent in the along-stream integration are comparable in magnitude to this imbalance. 
produces a substantial ageostrophic mean eddy flux. And finally, even if the Reynolds stress terms $\left(u, v_{\imath}\right)$ were exactly zero along streamlines, the model discretization produces finite derivatives in stream coordinates.

\subsubsection{Local Balance Along Streamlines.}

Although the global balance is dominated by wind stress, effects other than the wind stress may be a substantial part of the local dynamics. In Figure 4.7, the momentum balance along the $0.2 \mathrm{~m}$ height contour (fourth from the north in Figure 4.5) reveals dramatic shifts in the magnitude of the terms in the momentum balance. The flow appears to separate into two regimes: in relatively quiescent regions where the ocean is deep and bathymetry plays a negligible role, such as the region between $10^{\circ} \mathrm{E}$ and $60^{\circ} \mathrm{E}$, the terms in the momentum balance are small and vary gradually over large distances; here form drag roughly balances wind stress. In contrast, at three isolated topographic features, Kerguelen Island at $70^{\circ} \mathrm{E}$, Macquarie Ridge and Campbell Plateau at $170^{\circ} \mathrm{E}$, and Drake Passage at $60^{\circ} \mathrm{W}$, the terms are larger and more variable, and eddy fluxes and friction may be substantially larger than the wind stress. All of these topographic features extend to the ocean surface in certain locations, but are deeper than $1000 \mathrm{~m}$ through the central portion of the ACC flow. Nonetheless, their presence has a dramatic effect on the magnitude of the terms in the momentum balance.

The difference between regions near topography and deep mid-basin areas can be represented statistically by measuring the percentage of along-stream points at which at least $50 \%$ of the form drag is explained by the wind in the local balance: This is true at $81 \%$ of points in quiescent regions, but at just $30 \%$ of points in eddy active regions. Particularly in regions of substantial topographic variation, the mean and transient horizontal flux divergences, the mean vertical flux divergence, and horizontal viscosity all substantially influence the overall momentum balance. In other words, the wind is fairly constant along streamlines; in quiescent regions, 


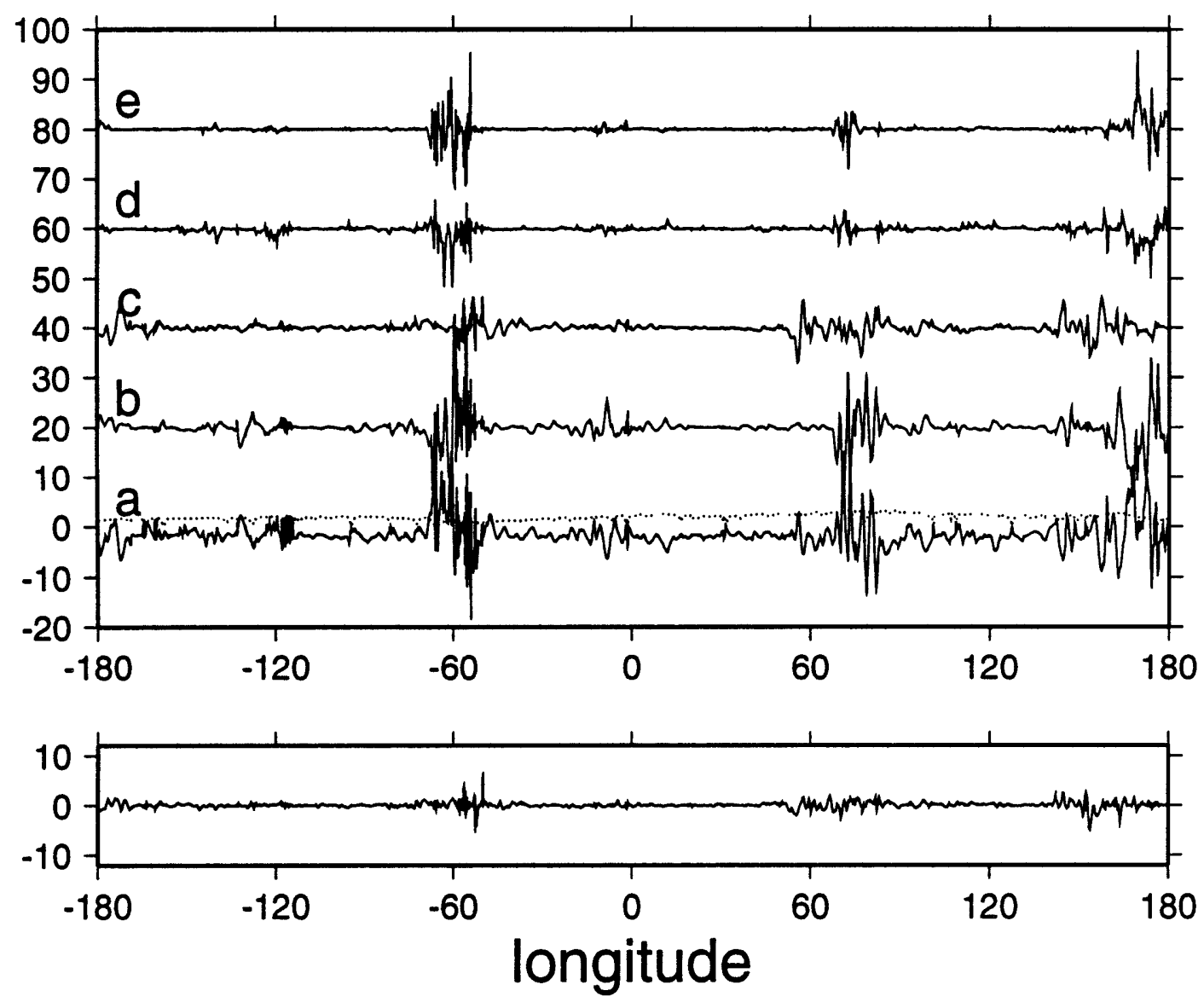

Figure 4.7: Vertically integrated momentum balance along the height contour 0.2 in the quarter-degree Semtner-Chervin model output in $\mathrm{cm}^{2} \mathrm{~s}^{-2}$. Upper panel shows (a) the ageostrophic Coriolis term, $-\int f v_{a g} d z$ which roughly represents the form drag and (dashed) wind stress, (b) the equatorward mean momentum flux divergence, (c) the equatorward eddy flux divergence, (d) the biharmonic friction, and (e) mean vertical eddy fluxes; terms are offset by 20 . The lower panel shows the residual imbalance, which is due to inaccuracies in computing the pressure gradient and eddy flux terms and acceleration due to curvature of the ACC path. 


\begin{tabular}{|c|c|c|c|}
\hline $\begin{array}{l}\text { Term } \\
\frac{\partial \overline{u v}}{\partial y}\end{array}$ & $\begin{array}{c}\text { All Points } \\
53 \%\end{array}$ & $\begin{array}{c}\text { Eddy Active Regions } \\
56 \%\end{array}$ & $\begin{array}{c}\text { Quiescent Regions } \\
52 \%\end{array}$ \\
\hline$\frac{\partial u^{\prime} v^{\prime}}{\partial y}$ & $35 \%$ & $29 \%$ & $36 \%$ \\
\hline$A_{m} \nabla^{4} \bar{u}$ & $19 \%$ & $20 \%$ & $19 \%$ \\
\hline$\frac{\partial \overline{u_{1}}}{\partial x_{2}}$ & $11 \%$ & $16 \%$ & $10 \%$ \\
\hline number of point & 16313 & 3601 & 12701 \\
\hline
\end{tabular}

Table 4.1: The contribution of each major term in the vertically integrated, timeaveraged along stream momentum equation. Values shown indicate the percentage of points along stream at which the indicated term accounts for over $50 \%$ of the residual difference between form drag and wind stress, that is points where $\left|\frac{\text { form drag-term }}{\text { form drag }}\right|<$ 0.5. Eddy active regions correspond to three topographic features: Kerguelen Island $\left(76^{\circ} \mathrm{E}\right.$ to $\left.82^{\circ} \mathrm{E}\right)$, Campbell Plateau $\left(145^{\circ} \mathrm{E}\right.$ and $\left.170^{\circ} \mathrm{E}\right)$, and Drake Passage $\left(70^{\circ} \mathrm{W}\right.$ to $45^{\circ} \mathrm{W}$ ). This statistical comparison, while unusual, effectively minimizes the impact of large outliers. The number of points at the bottom indicates the total number of along-stream values considered in each category.

the ageostrophic velocity locally balances the wind in the form of a surface Ekman transport, but it varies substantially in response to topographic features.

These differences might imply that the ACC dynamics near topographic features are substantially altered from those in broad ocean basins. To examine the relative importance of momentum flux divergences and friction in the along-stream momentum balance, each of the terms is compared with the residual difference between the wind stress and form drag. Although the magnitudes of fluctuations of each of the terms in Figure 4.7 differ substantially between quiescent regions and energetic regions, the actual partitioning of energy varies less substantially. Table 4.1 indicates a slight decrease in the importance of transient eddy fluxes and an increase in the importance of the mean vertical momentum flux divergence within eddy active regions, but the differences account for less than $10 \%$ of the variability. Thus the overall dynamical regime does not shift enormously between eddy active and quiescent regions, and the major difference appears to be the substantially greater kinetic 
energy levels in eddy active regions. Energy levels along streamlines will be addressed in greater detail in section 4.7 .

\subsubsection{Form Drag Along Streamlines.}

Although wind forcing is uniformly balanced by the ageostrophic portion of the Coriolis term along streamlines, the actual form drag is caused by individual ridges. Using (4.13), the effective form stress is estimated over roughly $10^{\circ}$ swaths in longitude. The results, illustrated in Figure 4.8, indicate that form stress can be attributed almost exclusively to three major topographic features, Drake Passage near $70^{\circ} \mathrm{W}$, Kerguelen Island, near $60^{\circ} \mathrm{E}$, and Campbell Plateau, around $170^{\circ} \mathrm{E}$. Smaller topographic features are not an apparent source of form drag in the numerical model output.

These results are consistent with Wang's [1993] analytic findings showing that for a channel of width $W$ and depth $H$, only ridges exceeding a critical height, $h_{c}=$ $\frac{\beta W H}{f_{o}}$, can substantially influence the total transport of 1-layer flow, because only for these ridges does the $\beta$-effect deflect all of the flow far enough meridionally to force it to form a frictional boundary current along the equatorward wall of the channel as shown in Figure 4.9. Thus only ridges exceeding $h_{c}$ are likely to be sources of form drag. While this narrow channel model may not accurately represent the ACC along most of its trajectory, each of the three topographic features which are sources of form drag in the Semtner-Chervin model are quite tall, breaching the surface in some locations, and they do confine the ACC to a narrow channel. Wang does not consider subsurface channels defined by deep topography.

In contrast to the form drag's localized response to three ridges in the upper panel of Figure 4.8, the ageostrophic velocity term in the lower panel responds uniformly to the wind; as discussed in section 4.2.1, it does not represent the form drag except when averaged around the entire circumpolar path and may be a misleading proxy for form drag. 


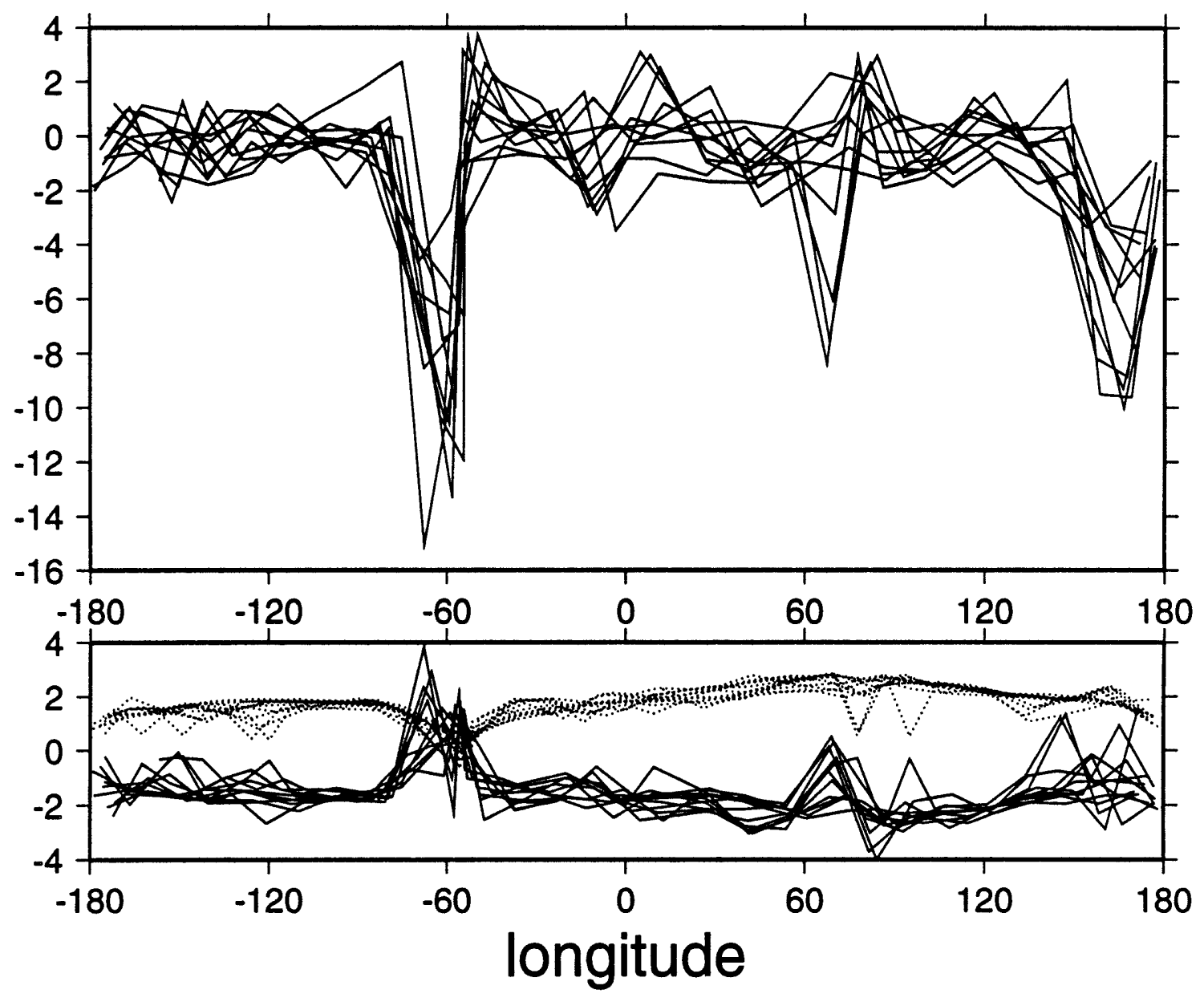

Figure 4.8: (Upper panel) Total vertically integrated form stress (as defined in (4.13), integrated along roughly $10^{\circ}$ wide swaths along streamlines in the ACC and divided by the length of the streamlines, in $\mathrm{cm}^{2} \mathrm{~s}^{-2}$. This provides a measure of the portion of form drag (averaged along streamlines) generated in each longitude region. Results for all height contours from $-0.5 \mathrm{~m}$ to $0.5 \mathrm{~m}$, as shown in Figure 4.5 are superimposed. (Lower panel) Wind stress (dashed line) and ageostrophic velocity term (solid line) for the same segments used in the upper panel indicate that the wind stress and oceanic response are fairly uniform, even though the actual form drag itself is concentrated at three ridges. 

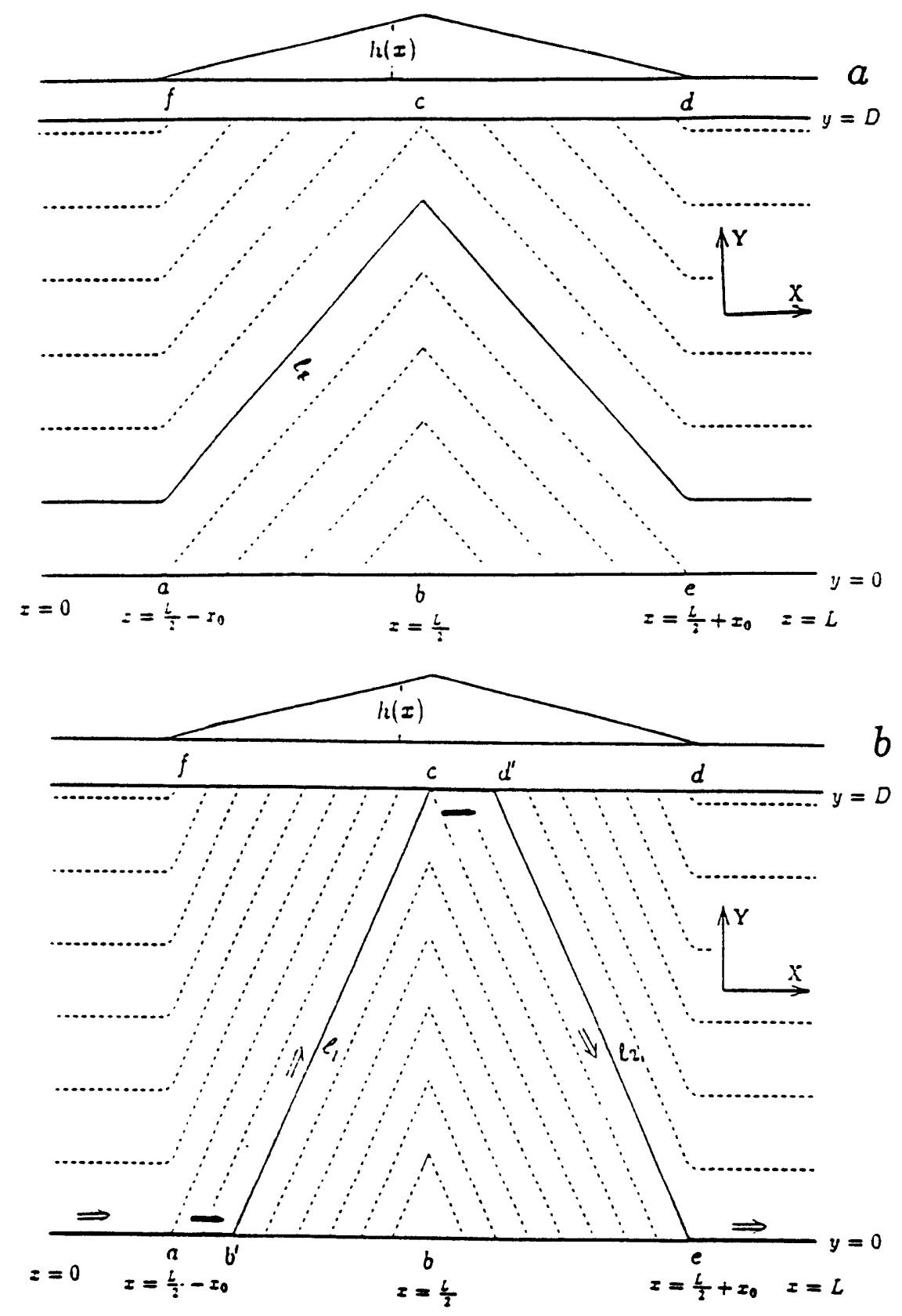

Figure 4.9: (a) Geostrophic contours for barotropic flow over a subcritical ridge ( $h_{\circ}<$ $h_{c}$ ) indicating that geostrophic flow is not frictionally reduced by the ridge. (b) Geostrophic contours for barotropic flow over a supercritical ridge $\left(h_{o}>h_{c}\right)$. Contours are deflected far enough meridionally that they encounter the northern wall of the channel and form a frictional boundary current between points $\mathrm{c}$ and d'. Reproduced from Wang [1993]. 


\subsection{Mid-Depth Momentum Balance}

\subsubsection{Zonally Averaged Balance}

At mid-depth, below the direct surface forcing and above topography, momentum transfers downward via interfacial form drag and meridionally, into and out of the ACC. Figure 4.10 shows the zonally averaged momentum balance at level 10 of the model, corresponding to $610 \mathrm{~m}$ depth. The dominant balance is between the ageostrophic Coriolis term and the mean equatorward eddy flux divergence; as in the vertically integrated zonally averaged case, standing eddies play a critical role in transferring momentum meridionally across the ACC. Biharmonic friction and transient eddy fluxes offer additional smaller contributions to the momentum balance. The FRAM model displayed a similar balance [Stevens and Ivchenko, 1994].

\subsubsection{Average Balance along Streamlines}

The apparent importance of stationary eddies is largely a result of the coordinate system used, as in the vertically integrated case. Transforming to stream coordinates on a single level is straightforward since the isobars (shown in Figure 4.11) are equivalent to geostrophic streamlines, and the cross-stream geostrophic velocity is trivially eliminated. The resulting momentum balance in Figure 4.12 identifies the processes most important for transferring momentum into and out of the circumpolar ACC. The results are similar to those found in the vertically averaged case. The largest terms are the ageostrophic Coriolis term, roughly representing interfacial form drag as discussed in section 4.2.1, and biharmonic friction. Transient eddy fluxes are substantially weaker than friction, and in these results are comparable in magnitude to the stationary eddy fluxes. The non-zero magnitude of the stationary eddy fluxes appears to contradict the predictions of Marshall et al. [1993] arguing that stationary eddies should have no impact on the cross-stream momentum flux; however, the size 

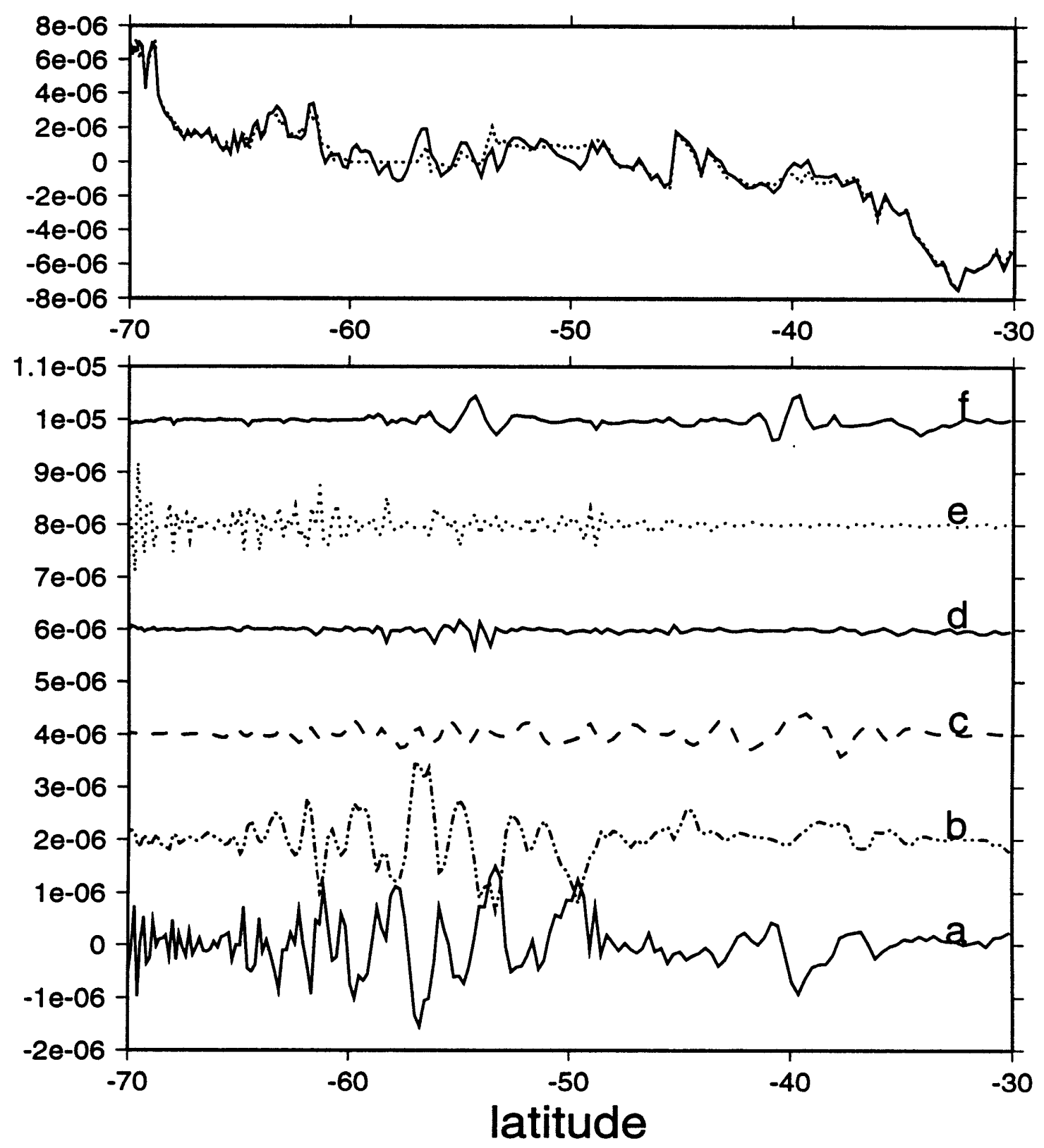

Figure 4.10: Zonally averaged momentum balance at level $10(610 \mathrm{~m})$ in the quarterdegree Semtner-Chervin model output in $\mathrm{cm} \mathrm{s}^{-2}$. Upper panel shows (solid line) the Coriolis term, $\frac{1}{L} \int f v d x$, and (dotted line) the pressure gradient term, $\frac{1}{L} \int f v_{g} d x$. The term $\int f v_{g} d x$ is zero for unblocked contours, since the pressure gradient term integrates to zero. Lower panel shows (a) the difference between the two, $\frac{1}{L} \int f v_{g}-$ $f v d x=-\frac{1}{L} \int f v_{a g} d x$, which is the form drag, (b) the mean equatorward momentum flux divergence, (c) the equatorward eddy flux divergence, (d) mean vertical eddy flux divergence, $(e)$ the biharmonic friction, and (f) the residual imbalance. Values are offset by $2 \times 10^{-6} \mathrm{~cm} \mathrm{~s}^{-2}$. 


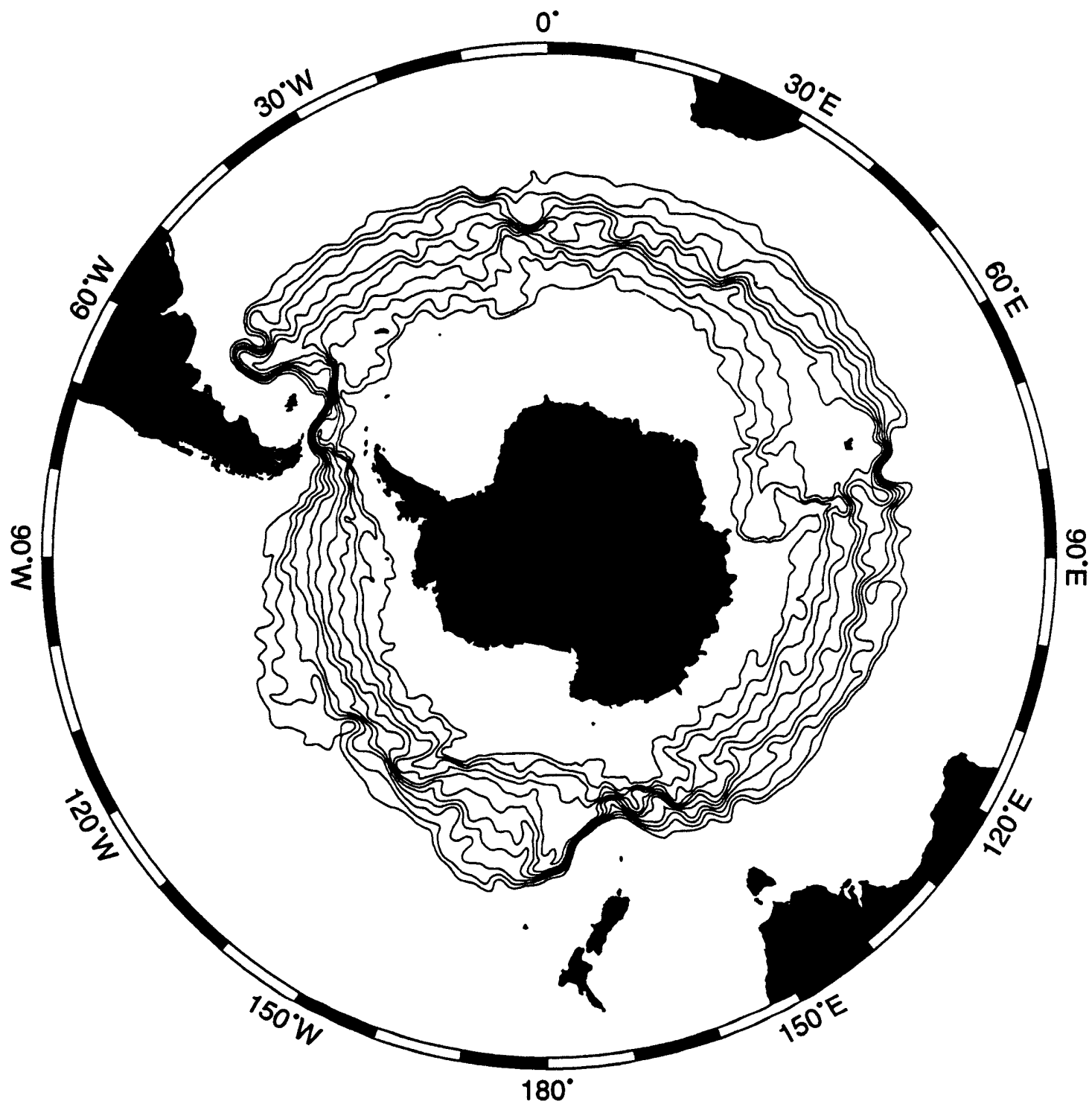

Figure 4.11: Continuous circumpolar pressure contours used to represent streamlines at level $10(610 \mathrm{~m})$ in the model output. Contour interval is $1 \times 10^{4}$ dynes $\mathrm{cm}^{-2}$; the densest contour line shown, at the southern limit of the ACC, corresponds to $6.018 \times 10^{7}$ dynes $\mathrm{cm}^{-2}$, and the northern limit is at $6.028 \times 10^{7}$ dynes $\mathrm{cm}^{-2}$. 

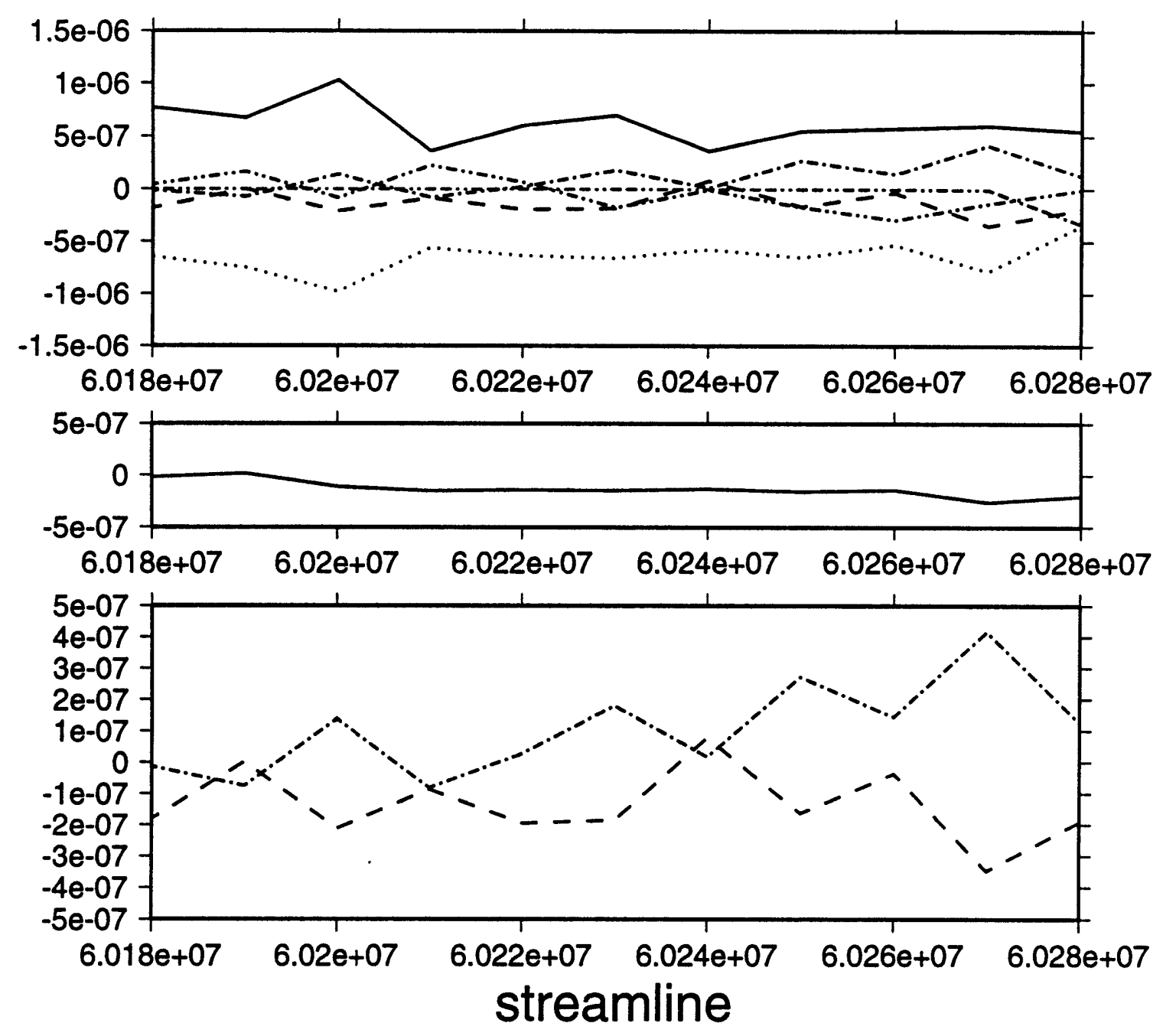

Figure 4.12: Momentum balance at level $10(610 \mathrm{~m})$ averaged along geostrophic streamlines in the quarter-degree Semtner-Chervin model output in $\mathrm{cm} \mathrm{s}^{\mathbf{- 2}}$. Upper panel shows (solid line) the Coriolis term, $-\frac{1}{L} \int f v d s$ which roughly represents the form drag, (dash double-dotted line) the mean equatorward momentum flux divergence, (dashed line) the equatorward eddy flux divergence, (dotted line) the biharmonic friction, (dash-dotted line) mean vertical eddy flux divergence, and (double dash-double dotted line) bottom friction. The middle panel shows the residual imbalance. The lower panel indicates (dashed line) the equatorward eddy flux divergence and (dash-dotted line) the mean vertical eddy flux divergence, which are discussed in the text. 
of the term is probably an error due to the transforming coordinates of the model data on a discretized grid and is not representative of mean cross-stream momentum fluxes in the ocean. Thus, these results suggest that horizontal viscosity, rather than eddy processes, is the leading process transferring momentum across the ACC.

Horizontal eddy dissipation schemes are widely debated in numerical modeling, so the importance of horizontal viscosity in transferring momentum across the model ACC to balance form drag presents a troublesome difficulty. Recent work by Danabasoglu et al. [1994] has investigated an parameterization for the horizontal tracer diffusion in the Cox-Bryan model, run with coarser resolution than that used by Semtner and Chervin. They found that including an along-isopycnal mixing term substantially improved the model's ability to reproduce large-scale observations. Although they did not consider finer resolution variations of the same problem and did not examine alternative representations of viscosity, their results suggest that horizontal viscosity could be an inadequate representation of sub-grid scale eddy processes in the ocean and might produce effects.

Some consideration of the ACC model physics helps explain why cross-stream eddy viscosity exceeds the cross-stream eddy fluxes, at mid-depth and in the vertically averaged model. To replace the northward surface Ekman transport, water must continually upwell from the deep Southern Ocean. This requires a deep poleward flow. Since the ACC has no continental boundaries, this may occur as a geostrophic flow below the sill depth or as an ageostrophic flow anywhere in the water column. Form drag, the dominant means to balance wind stress, is associated with deep geostrophic flow, while eddy processes and dissipation which may act at any depth, are associated with ageostrophic flow. Mesoscale eddy flux divergences may not be adequately represented by the model resolution. The subgrid scale parameterization of eddy flux divergences is presented by horizontal diffusive processes which tend to smooth out velocity and density fields, decreasing the sharpness of fronts. This has two effects. First, in the deep ocean, it will tend to create a very homogeneous, horizon- 
tally non-divergent velocity field with minimal vertical velocities; this means little mass transfers from below the sill depth to the surface. Second, diffusive fluxes are strongest where gradients are largest, across velocity jets. Since velocity jets are associated with tilting isopycnals, this is equivalent to a substantial cross-isopycnal viscosity. Thus, diffusive processes tend to inhibit deep geostrophic flow and increase mid-depth cross-stream momentum transfer. The net effect is to replace adiabatic upwelling from the deep ocean with cross-stream momentum transfer, removing momentum from the ACC by transferring it horizontally rather than vertically. (The eddy processes which viscosity is intended to model have a similar effect, but they may be more surface intensified and better able to transfer momentum along isopycnal surfaces.)

To begin to understand why eddy viscosity exceeds cross-stream eddy fluxes in this model, reexamine the lower panel of Figure 4.12: in a rough sense, the mean vertical eddy flux, $\frac{\partial(\bar{u} \bar{w})}{\partial z}$, balances the transient horizontal eddy flux, $\frac{\partial \overline{u^{\prime} v^{\prime}}}{\partial y}$. Therefore the transient eddies balance the non-rotational portion of the interfacial form drag divergence $\left(\frac{\partial(\bar{u} \bar{w})}{\partial z}\right)$ but not the rotational interfacial form drag divergence $\left(f v_{a g}\right)$. (The distinction is discussed in section 4.2.1). The result may be an indication that the along-stream transport, $u$, acts as a conservative tracer at this depth in the model. If the ACC is like a continuous river, carrying a constant volume of fluid at each depth in the model, then the conservation expression applies:

$$
\oint \frac{D \bar{u}_{s}}{D t} d x_{s}=\oint \overline{\mathbf{u} \cdot \nabla u_{s}} \Delta x_{s} \approx 0 .
$$

The total derivative $D u_{s} / D t$ represents the change in along-stream speed of a water parcel contained within the ACC. Integrated along a circumpolar path, this expression indicates that the total transport on a streamline does not vary as a function of time, though it may undergo regional fluctuations. Combining (4.18) with the continuity equation and integrating around a closed geostrophic streamline produces:

$$
\frac{\partial \overline{u^{\prime} v^{\prime}}}{\partial y}+\frac{\partial(\bar{u} \bar{w})}{\partial z} \approx 0
$$


which conforms to the model result shown in Figure 4.12. The observed balance between eddy stress divergence terms therefore suggests that transport is conserved along streamlines at mid-depth. Biharmonic friction differs from the advective processes by not adhering to this conservation property; instead large friction forces a large ageostrophic velocity term, thus transferring momentum from interfacial form drag to horizontal friction, by driving a cross-stream velocity. In future modeling efforts, establishing a frictional term which more closely matches the properties of the ACC may require developing a more realistic sub-grid scale parameterization.

These results show that the vertical divergence of form drag is dominated by biharmonic friction rather than cross-stream eddy flux divergences, but they do not address the interfacial form drag itself, which is associated with the buoyancy flux. The question of whether the cross-stream heat flux is predominantly due to diffusion or eddy fluxes is not addressed in this analysis and is beyond the scope of this thesis. Previous work on heat fluxes in the FRAM model has shown that the meridional heat flux is due to stationary eddies in zonal coordinates [Saunders and Thompson, 1992] and transient eddies in stream coordinates [Thompson, 1993]. However, neither investigation examined the diffusion terms in the buoyancy flux equations. The nature of the meridional buoyancy flux and corresponding interfacial form drag processes remains a subject for future investigation.

\subsubsection{Balance along Streamlines}

Examining the momentum balance along a single streamline, corresponding to the isobar, $p=6.020 \times 10^{7}$ dynes $\mathrm{cm}^{-2}$, allows examination of local processes. In Figure 4.13 dramatic shifts in the magnitude of the balance terms are associated with major topographic features, as was also indicated in Figure 4.7. The relative importance of terms in eddy active and quiescent regions is compared in Table 4.2, using the same statistical measure as in the vertically averaged case. At least $50 \%$ of the form drag is explained by mean and eddy effects at $57 \%$ of points located in quiescent regions, 

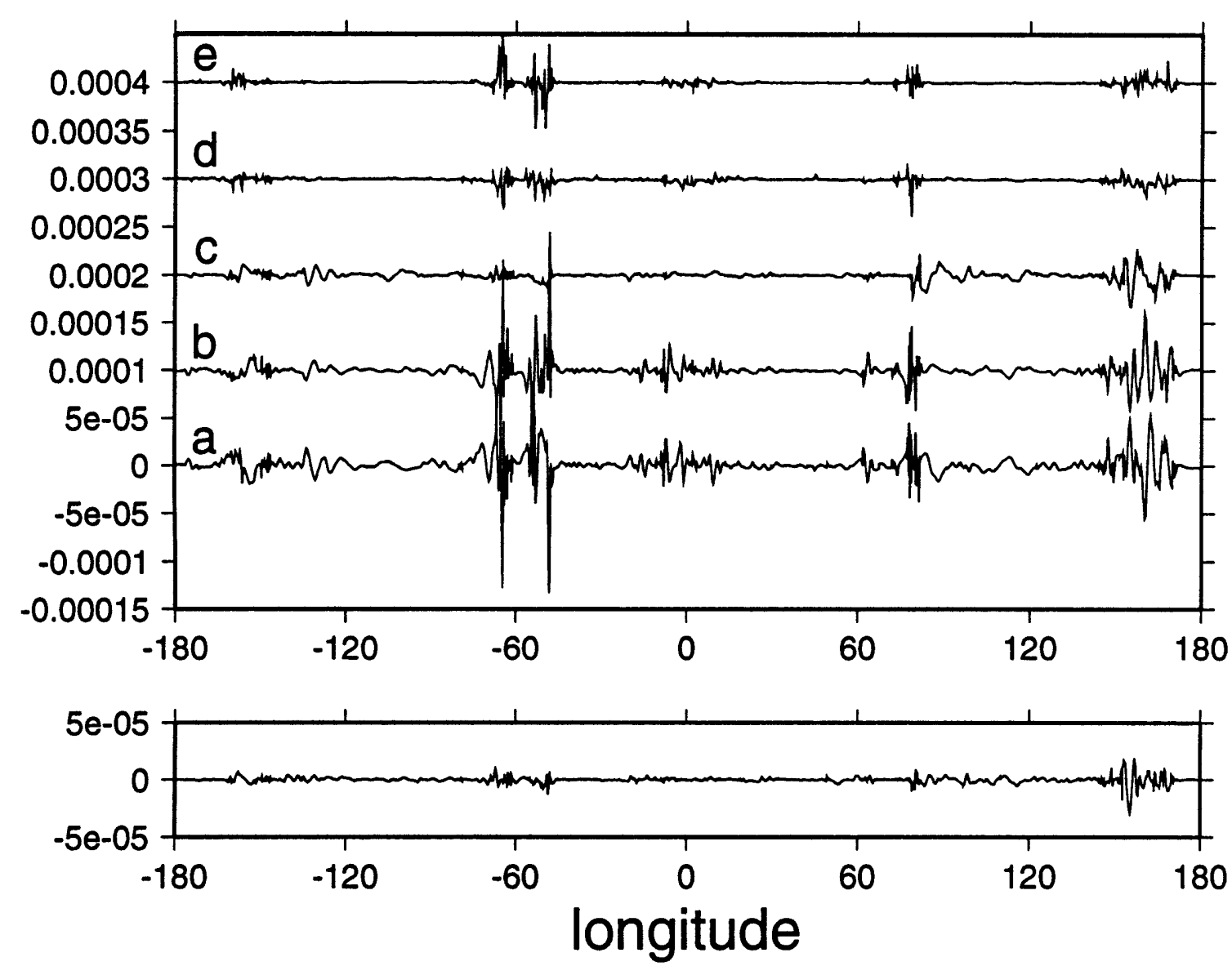

Figure 4.13: Momentum balance at level $10(610 \mathrm{~m})$ along the geostrophic streamlines corresponding to $p=6.020 \times 10^{7}$ dynes $\mathrm{cm}^{-2}$ in the quarter-degree Semtner-Chervin model output in $\mathrm{cm} \mathrm{s}^{-2}$. Upper panel shows (a line) the Coriolis term, $-f v$ which represents the form drag, (b) the equatorward mean momentum flux divergence, (c) the equatorward eddy flux divergence, (d) the biharmonic friction, and (e) mean vertical eddy fluxes; terms are offset by $1 \times 10^{-4}$. The lower panel shows the residual imbalance, which is due to inaccuracies in computing the pressure gradient and eddy flux terms and acceleration due to curvature of the ACC path. 


\begin{tabular}{|lccc|}
\hline Term & All Points & Eddy Active Regions & Quiescent Regions \\
$\frac{\partial \overline{u v}}{\partial y}$ & $43 \%$ & $44 \%$ & $43 \%$ \\
$\frac{\partial u^{\prime} v^{\prime}}{\partial y}$ & $22 \%$ & $17 \%$ & $24 \%$ \\
$\frac{\partial \overline{u v}}{\partial v}+\frac{\partial \overline{u^{\prime} v^{\prime}}}{\partial y}$ & $56 \%$ & $51 \%$ & $57 \%$ \\
$A_{m} \nabla^{4} \bar{u}$ & $8 \%$ & $10 \%$ & $8 \%$ \\
$\frac{\partial \bar{u} w}{\partial z}$ & $7 \%$ & $9 \%$ & $6 \%$ \\
\hline number of points & 16978 & 3616 & 13349 \\
\hline
\end{tabular}

Table 4.2: The contribution of each major term in the level $10(610 \mathrm{~m})$, time-averaged along-stream momentum equation. See Table 4.1 for further explanation.

and at $51 \%$ of points in eddy intense regions. Most of the slight difference occurs because the transient eddy fluxes have a smaller impact on the momentum balance near the isolated topographic features while the effect of mean eddy fluxes is nearly constant. The decrease in transient eddy effects is partially balanced by an increase in the mean vertical flux divergence. Since the residual imbalance increases slightly in eddy active regions, the disparity may result predominantly from errors in computing transient eddy fluxes. The differences between the two types of regions, however, are relatively small; as in the vertically integrated case, the major distinguishing features between areas where topography affects the flow and quiescent regions appear to be in the magnitude of the momentum rather than in the partitioning between terms in the equations.

\subsection{Surface Momentum Balance}

\subsubsection{Zonally Averaged Balance}

In contrast with the relatively calm mid-depth ocean, in the upper $100 \mathrm{~m}$ (4 levels) of the model, surface wind stress, $\frac{\tau^{*}}{\rho_{0}}$, forces a strong northward Ekman transport, as shown in Figure 4.14. This northward transport results in a corresponding east- 

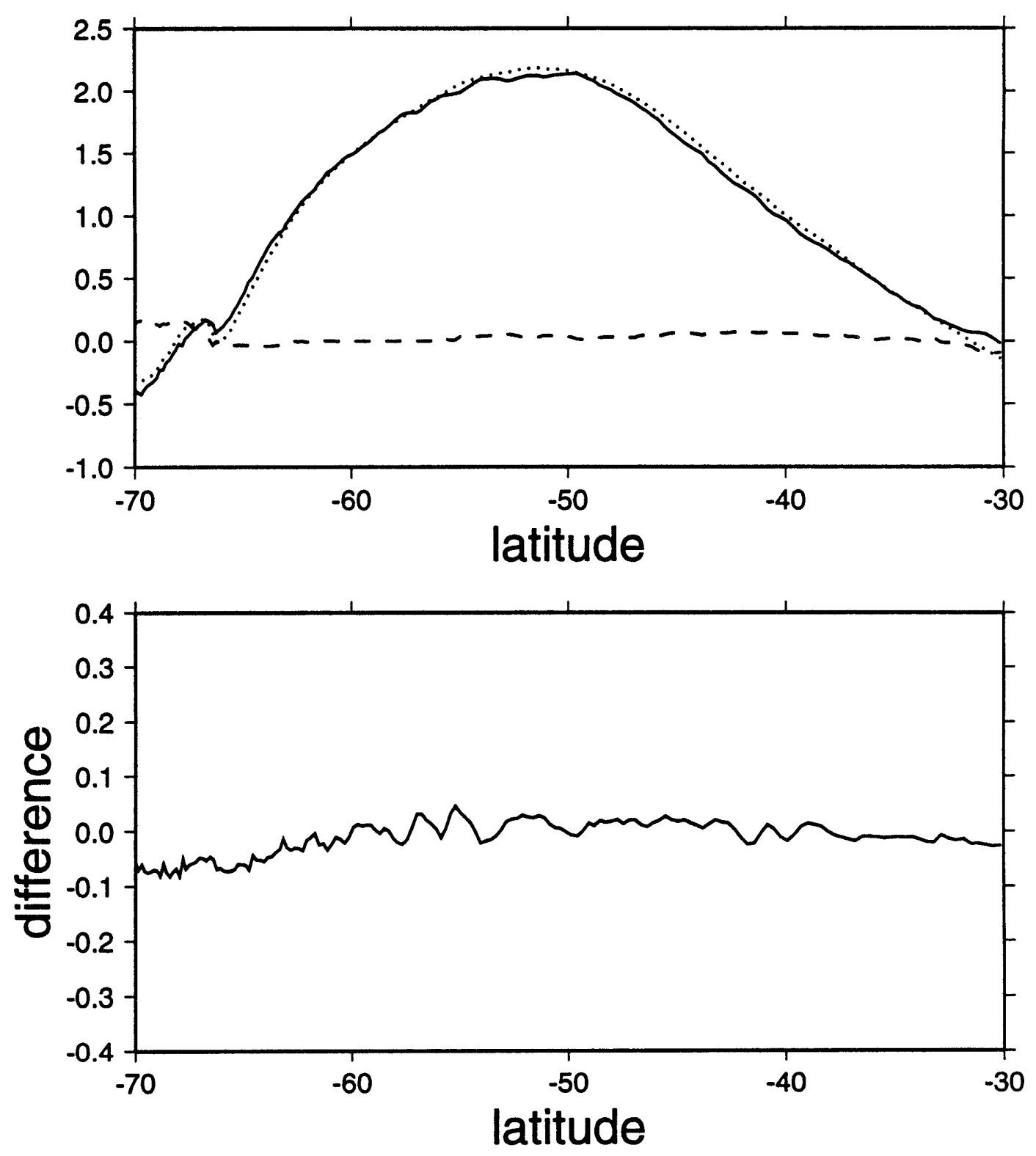

Figure 4.14: Vertically integrated over the upper $100 \mathrm{~m}$ (4 levels) and zonally averaged geostrophic balance in the quarter-degree Semtner-Chervin model output in $\mathrm{cm}^{2} \mathrm{~s}^{-2}$. Upper panel shows (solid line) the Coriolis term $\frac{1}{L} \iint f v d x d z$, (dashed line) the pressure gradient term, $\frac{1}{L} \iint \frac{-1}{\rho_{0}} \frac{\partial_{p}}{\partial x} d x d z$ and (dotted line) the integrated stress term $\frac{1}{L} \iint \mu \frac{d^{2} u}{d z^{2}} d x d z \approx \int \frac{\tau^{*}}{p_{0}} d x$. Lower panel shows the residual imbalance. 
west tilting of isopycnals to achieve a geostrophic return flow; this sets up interfacial form drag which transfers wind energy to depth where it is balanced via topographic form drag. The difference between wind forcing and Ekman transport is primarily due to surface layer errors in the vertical viscosity and in the precise recomputation of the wind forcing rather than physically significant phenomena. Therefore the unexplained portion of the momentum balance will be set to zero for the remainder of this discussion.

The adjusted difference between wind forcing and the Ekman response represents the small portion of the wind energy which is transferred laterally to be balanced by the forcing terms in the momentum balance, as shown in Figure 4.15. The surface is no different than the vertically integrated and mid-depth cases considered earlier: at most latitudes in the circumpolar region, the interfacial form drag divergence (line a) balances the mean momentum flux divergence (line $b$ ). Thus the wind inputs momentum and what momentum is not fluxed vertically by interfacial processes is transferred meridionally by standing eddies. Transient momentum flux divergences and horizontal viscosity are about an order of magnitude smaller than the standing eddy flux divergences in Figure 4.15.

\subsubsection{Average Balance along Streamlines}

The role of standing eddies is largely an artifact of using zonal coordinates. Averaging along stream coordinates (shown in Figure 4.16) illustrates how momentum is fluxed across the ACC (Figure 4.17). Along streamlines, the dominant balance is between the Coriolis term and wind stress, as predicted by the theory for surface Ekman layers. The portion of the wind stress not accounted for by the Ekman transport is transferred laterally out of the ACC by a combination of horizontal viscosity, mean horizontal momentum flux divergence, transient horizontal momentum flux divergence, and mean vertical momentum flux divergence. Horizontal friction plays a less significant part than it does at mid-depth while the strong cross-stream ageostrophic 


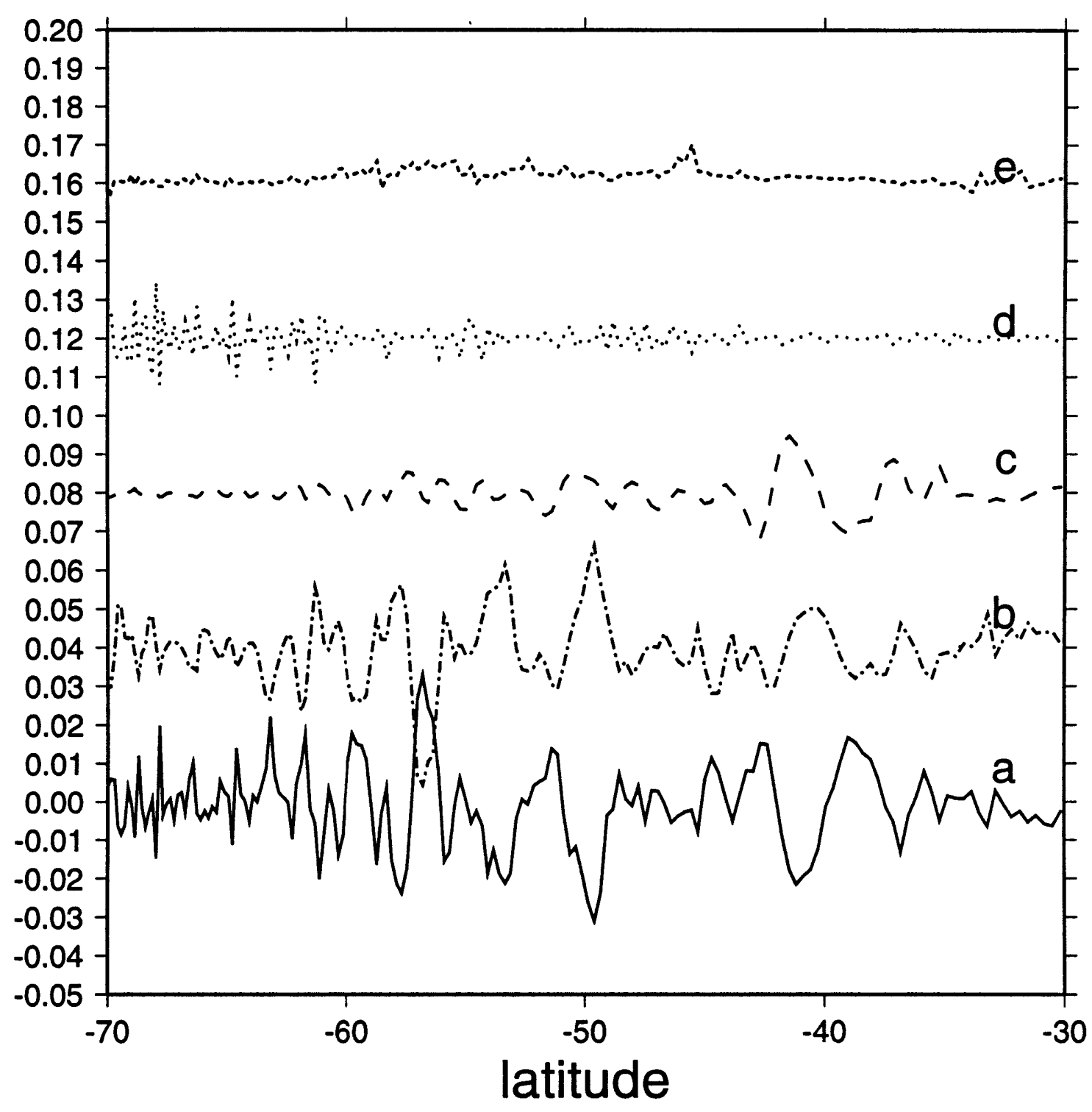

Figure 4.15: Vertically integrated over the upper $100 \mathrm{~m}$ (4 levels) and zonally averaged geostrophic balance in the quarter-degree Semtner-Chervin model output in $\mathrm{cm}^{2} \mathrm{~s}^{-2}$. Terms represented are (a) the portion of the wind stress not accounted for by the Coriolis term or form drag, $\left(\frac{1}{L} \iint f v d x d z-\frac{1}{L} \iint \frac{-1}{\rho_{0}} \frac{\partial \bar{p}}{\partial x}+\mu \frac{d^{2} \bar{u}}{d z^{2}} d x d z\right)$, (b) the mean equatorward momentum flux divergence $\left(\frac{1}{L} \iint \frac{\partial \overline{u v}}{\partial y} d x d z\right)$, (c) the equatorward eddy flux divergence $\left(\frac{1}{L} \iint \frac{\partial \overline{u^{\prime} v^{\prime}}}{\partial y} d x d z\right)$, (d) biharmonic friction $\left(\frac{1}{L} \iint A_{m} \nabla^{4} \bar{u} d x d z\right)$, and (e) mean vertical flux divergence. Terms are offset by $0.04 \mathrm{~cm}^{2} \mathrm{~s}^{-2}$. 


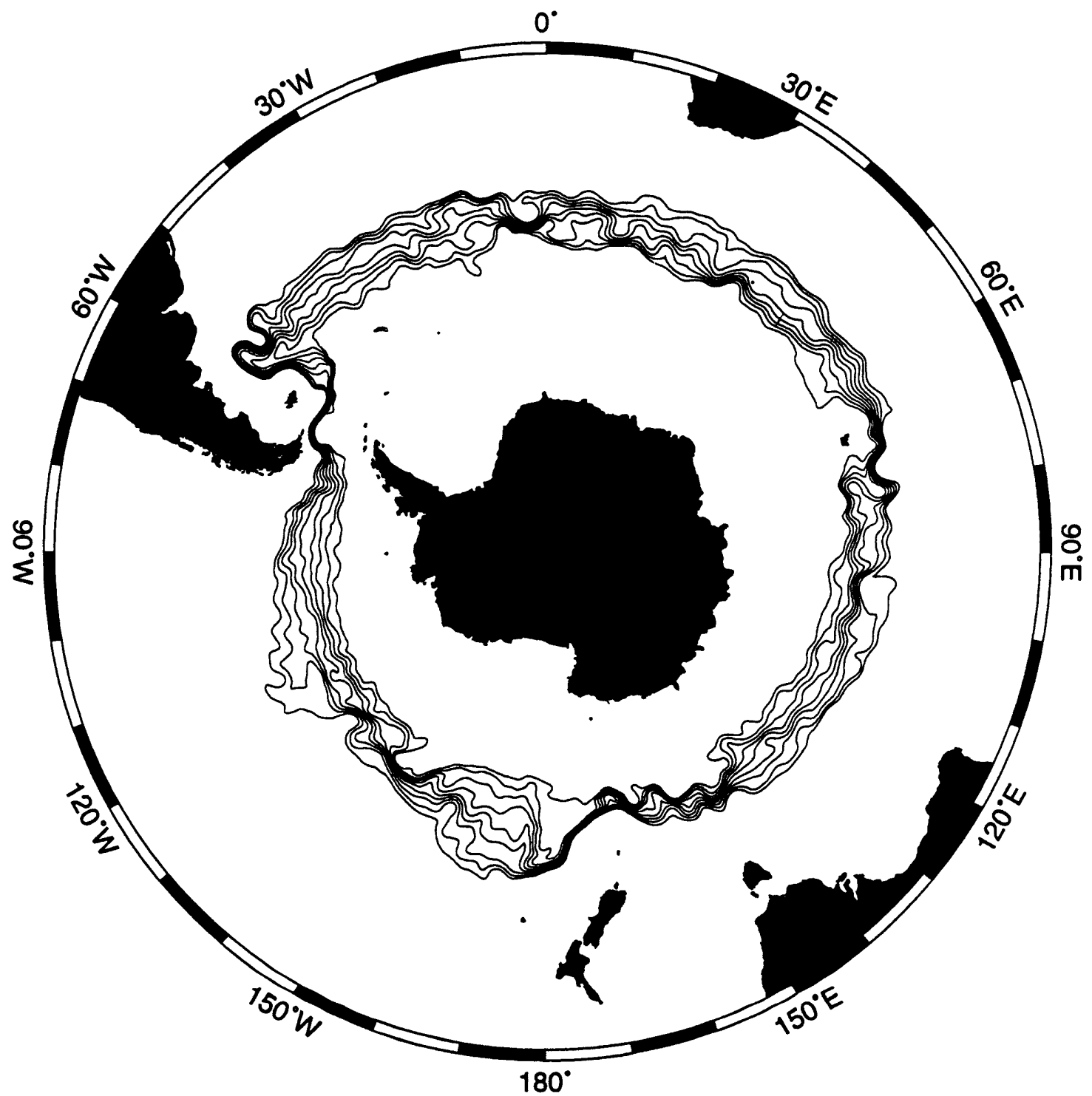

Figure 4.16: Integrated pressure contours used for mapping momentum balance for upper $100 \mathrm{~m}$ in stream coordinates. Contours extend from $4.81 \times 10^{8}$ to $4.89 \times 10^{8} \mathrm{~g}$ $\mathrm{m} \mathrm{cm}^{-1} \mathrm{~s}^{-2}$. The contour interval is $10^{6} \mathrm{~g} \mathrm{~m} \mathrm{~cm}^{-1} \mathrm{~s}^{-2}$. 


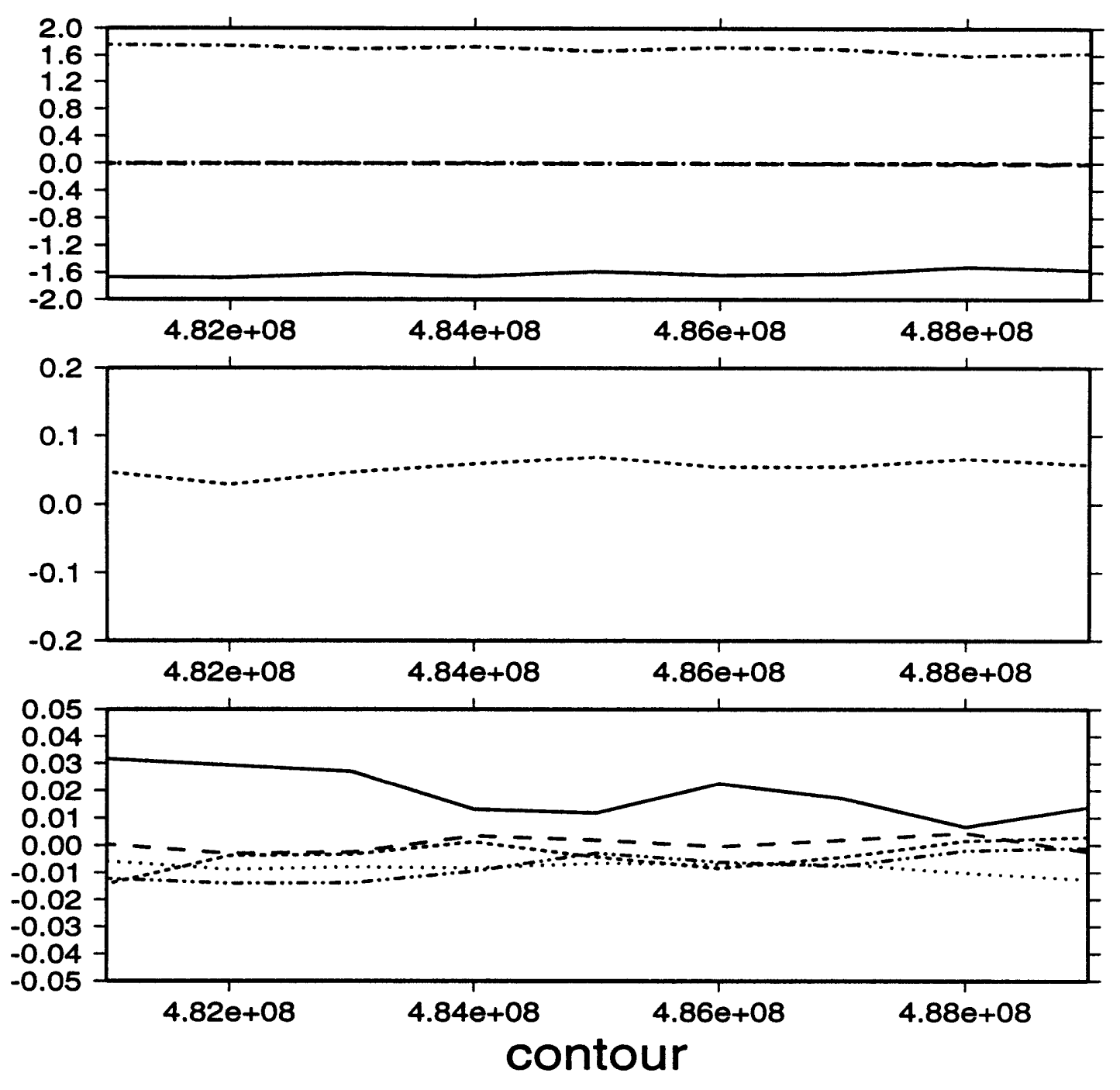

Figure 4.17: Momentum balance integrated vertically and averaged along streamlines for the top $100 \mathrm{~m}$ (4 levels) of the quarter-degree Semtner-Chervin model output in $\mathrm{cm}^{2} \mathrm{~s}^{-2}$. Upper panel shows (solid line) the Coriolis term $\left(\frac{1}{L} \iint f v d s d z\right)$ rotated into stream coordinates, (dash-dot line) the mean wind stress $\left(\frac{1}{L} \iint \mu \frac{\partial^{2} u}{\partial z^{2}} d s d z\right)$. All other terms are plotted but are indistinguishable from zero. The middle panel shows the residual imbalance. The lower panel shows the momentum balance when the surface Ekman transport has been removed. Terms represented are (solid line) the difference between wind stress and Coriolis, corrected for the residual misfit $\left(\frac{1}{L} \iint \mu \frac{\partial^{2} \bar{u}}{\partial z^{2}}+f v-\right.$ misfit $d s d z$ ), (dash double-dotted line) the mean equatorward momentum flux divergence $\left(-\frac{1}{L} \iint \frac{\partial \overrightarrow{v v}}{\partial y} d s d z\right)$, (long dashed line) the equatorward eddy flux divergence $\left(-\frac{1}{L} \iint \frac{\partial \bar{u}^{\prime} v^{\prime}}{\partial y} d s d z\right)$, (dotted line) the biharmonic friction term $\left(\frac{1}{L} \iint A_{m} \nabla^{4} \bar{u} d s d z\right)$, and (short dashed line) mean vertical flux divergence. 
velocity permits mean horizontal eddy fluxes to be large. Eddy flux divergence is the smallest of the terms, suggesting that transient processes do not have a strong impact on the model momentum balance at the ocean surface.

\subsubsection{Balance along Streamlines}

In the local balance along streamlines, the ageostrophic velocity term varies substantially, because the strong surface Ekman flux is at right angles to the wind, but is not oriented in any particular direction relative to the geostrophic velocity (Figure 4.18). The dominant balance represents a surface Ekman transport: surface wind stress is balanced by the ageostrophic portion of the Coriolis term. The difference between the wind stress and Ekman response, representing the horizontal transfer of momentum, is largely balanced by the mean momentum flux divergence. This term, which is small in the mid-depth ocean, is non-zero in the upper ocean because of the large ageostrophic cross-stream velocity driven by the wind. Mean vertical eddy fluxes and cross-stream transient eddy fluxes are slightly smaller, while viscosity appears to play a limited role in the momentum balance.

The partitioning of terms in the momentum balance, summarized in Table 4.3, does not change substantially between regions of topographic influence and more quiescent regions. As in the vertically integrated and mid-depth cases, the major variation with topography can be viewed as a shift from high eddy kinetic energy to low eddy kinetic energy without alteration of the dominant momentum balance.

\subsection{Surface Eddy Kinetic Energy}

Increased eddy kinetic energy near topographic features may be a result of topographic lee waves and may feed greater instabilities, but it does not appear to alter the overall momentum balance. Since the major along-stream variations in the mo- 

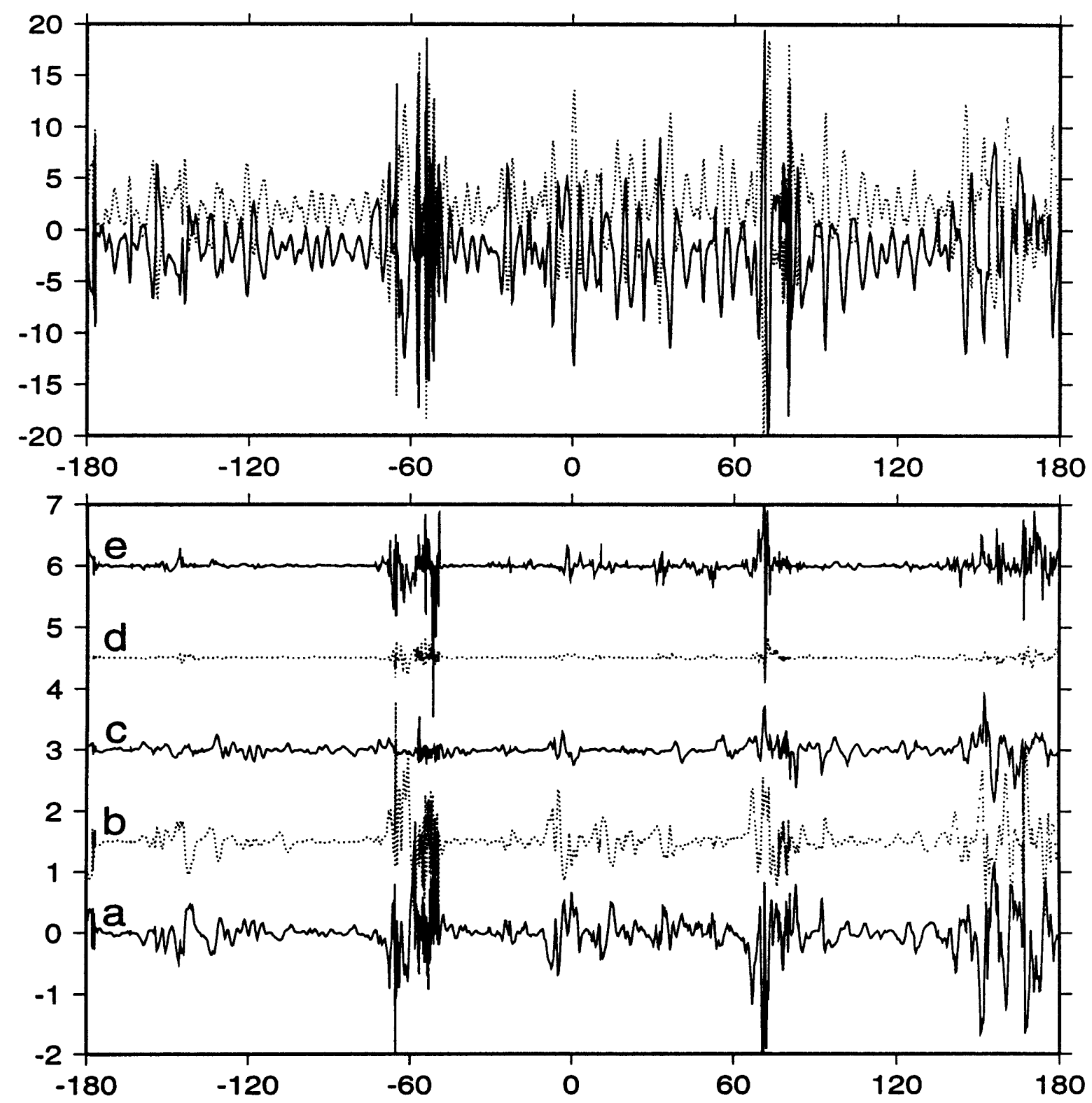

Figure 4.18: Momentum balance for upper $100 \mathrm{~m}$, along the pressure contour $4.84 \times$ $10^{8}$ dynes $\mathrm{cm}^{-2} \mathrm{~m}$ in the quarter-degree Semtner-Chervin model output in $\mathrm{cm}^{2} \mathrm{~s}^{-2}$. Upper panel shows the Coriolis term, $f \bar{v}$ and (dotted) surface wind stress $\left(\mu \frac{\partial^{2} \bar{u}}{\partial z^{2}}\right)$ adjusted to show a zero residual imbalance. Lower panel shows (a) the portion of wind stress not accounted for by the Coriolis term, (b) the equatorward mean momentum flux divergence, (c) the equatorward eddy flux divergence, (d) the biharmonic friction, and (e) mean vertical eddy fluxes; terms are offset by 1.5 . 


\begin{tabular}{|lccc|}
\hline Term & All Points & Eddy Active Regions & Quiescent Regions \\
$\frac{\partial \overline{u v}}{\partial y}$ & $41 \%$ & $43 \%$ & $40 \%$ \\
$\frac{\partial u^{\prime} v^{\prime}}{\partial y}$ & $25 \%$ & $21 \%$ & $27 \%$ \\
$\frac{\partial \bar{u} v}{\partial y}+\frac{\partial u^{\prime} v^{\prime}}{\partial v}$ & $67 \%$ & $63 \%$ & $69 \%$ \\
$A_{m} \nabla^{4} \bar{u}$ & $4 \%$ & $6 \%$ & $4 \%$ \\
$\frac{\partial \bar{u} w}{\partial z}$ & $12 \%$ & $12 \%$ & $12 \%$ \\
\hline number of points & 1485 & 380 & 1104 \\
\hline
\end{tabular}

Table 4.3: The contribution of each major term in the upper $100 \mathrm{~m}$ of the timeaveraged along stream momentum equation, along the isobar $p=4.84 \times 10^{8} \mathrm{~g} \mathrm{~m}$ $\mathrm{cm}^{-1} \mathrm{~s}^{-2}$. See Table 4.1 for further explanation.

mentum balance appear to be in the amplitude of the fluctuations rather than in the actual partitioning of momentum between the terms, changes in energy serve as a convenient indicator of the major momentum variations for comparison with other measurements. Eddy kinetic energy has the advantage that in addition to being easily computed from model output, it may also be estimated from altimeter data.

Detailed analyses by Morrow et al. [1992] and Johnson et al. [1992] have used crossover techniques for estimating eddy kinetic energy and Reynolds stresses from altimeter data. Here, a slightly different methodology has been developed. Rather than confining investigation to crossover points and temporally interpolating to match ascending and descending measurements, the along track variability data were objective mapped using decorrelation scales estimated directly from the data. The resulting regularly gridded instantaneous sea surface height maps include error estimates. Eddy kinetic energy and Reynolds stress estimates computed from the gridded data are generally consistent with the results of Morrow et al. [1992]. Error estimates are discussed in section 3.6 .

Numerical models have historically tended to underestimate surface kinetic energy relative to altimeter measurements. Wilkin and Morrow [1994] found that on average surface eddy kinetic energies estimated from the altimeter were a factor of 6 greater than those computed by the half-degree model. In contrast, in this analysis, 


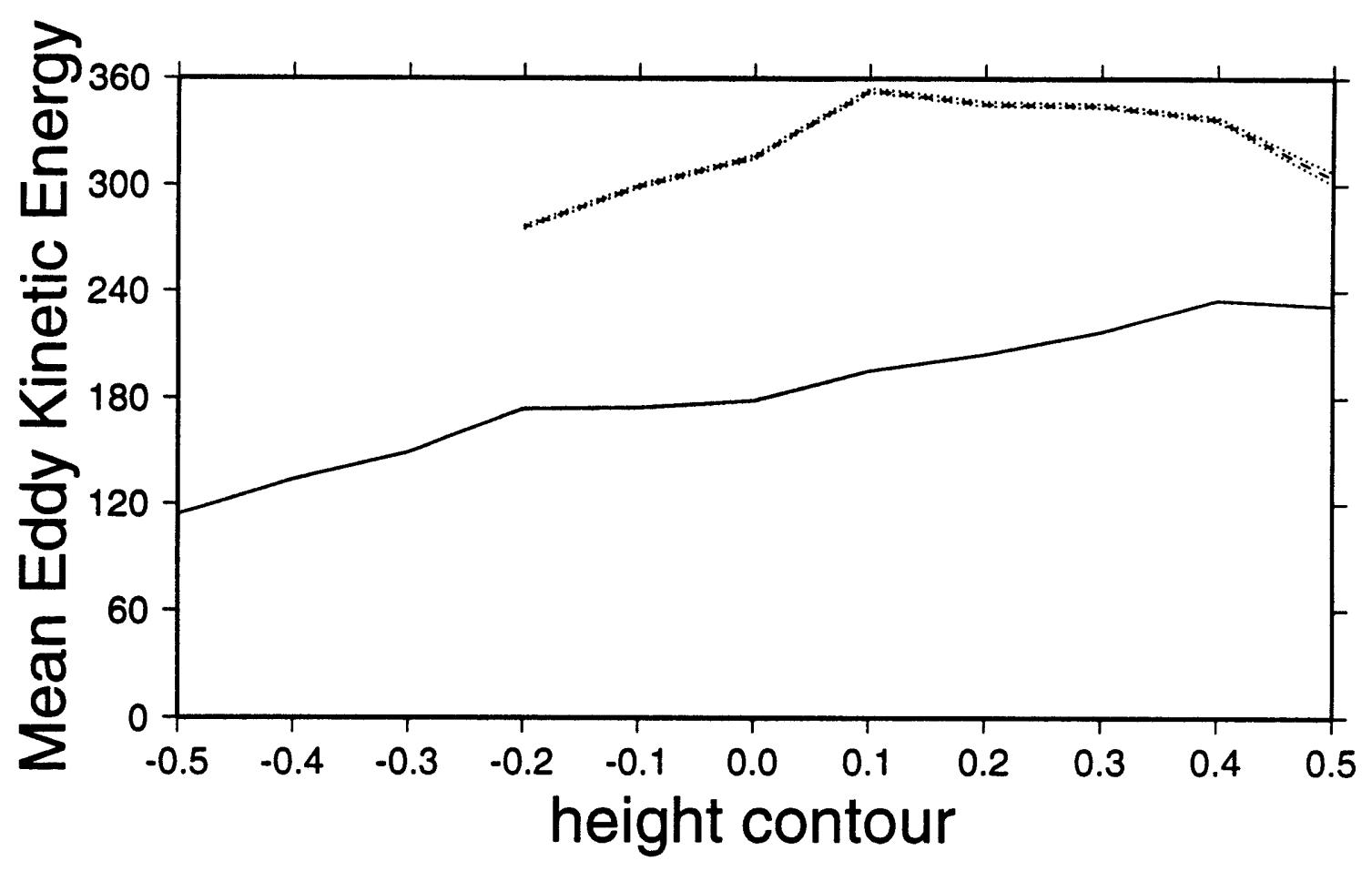

Figure 4.19: Eddy kinetic energy averaged along surface height contours. Solid line indicates energy in the quarter-degree Semtner-Chervin model output in $\mathrm{cm}^{2} \mathrm{~s}^{-2}$. Dashed line is the same quantity in altimeter data, with error limits indicated by dotted lines.

the discrepancy is reduced to a factor of 2, suggesting that the finer resolution of the quarter-degree model more effectively represents eddy processes. In the primary region for the data analysis, between $45^{\circ} \mathrm{S}$ and $60^{\circ} \mathrm{S}$, the mean eddy kinetic energy measured by the altimeter is $1.5 \times 10^{-2} \mathrm{~m}^{2} \mathrm{~s}^{-2}$, compared with $7.2 \times 10^{-3} \mathrm{~m}^{2} \mathrm{~s}^{-2}$ in the model output. Eddy kinetic energy is greatest along the axis of the ACC. In these energetic regions, Wilkin and Morrow [1994] found closer agreement, with the ratio of altimeter to model eddy kinetic energy reduced to between 2.2 and 4 . This analysis shows similar results: averaged along streamlines, eddy kinetic energy measured by the altimeter is about 1.6 times greater than that found in the model, as indicated in Figure 4.19. Like the FRAM model results shown by Ivchenko et al. [1994, Figure 8], the Semtner-Chervin model indicates a linear increase in eddy kinetic energy with sea surface height, but this version of the model is roughly twice as 
energetic. Neither model captures the eddy kinetic energy peak seen by the altimeter at the $0.1 \mathrm{~m}$ height contour, but the differences may be due to the broader nature of the ACC in portions of the model output. This peak in eddy kinetic energy near the axis of the Subantarctic Front suggests that the meandering ACC fronts themselves may account for a substantial portion of eddy kinetic energy in the Southern Ocean.

The results of the along-stream momentum balance in the preceding sections show that eddy kinetic energy varies substantially along streamlines, as shown in Figure 4.20. In the numerical model output, eddy kinetic energy has a relatively small background value and peaks at three major locations associated with topographic obstruction of the flow: Drake Passage $\left(60^{\circ} \mathrm{W}\right)$, Kerguelen Plateau $\left(70^{\circ} \mathrm{E}\right)$, and Campbell Plateau $\left(170^{\circ} \mathrm{E}\right)$. Eddy kinetic energy measured by the altimeter follows a similar pattern to that in the numerical model output. Energy levels are relatively low in quiescent deep ocean areas, but rise dramatically at topographic features. However, unlike the model results which suggest that only three topographic features are important in the momentum balance, the altimeter data show a broader energy peak at Drake Passage $\left(60^{\circ} \mathrm{W}\right)$, and increased energy near the Crozet Plateau $\left(40^{\circ} \mathrm{E}\right)$ and at the Eltanin and Udintsev Fracture Zones in the central Pacific $\left(140^{\circ} \mathrm{W}\right)$. The northern side of the ACC also shows increased energy near $140^{\circ} \mathrm{E}$, where the SAF crosses over the Indian-Antarctic Ridge. Thus, in addition to the three major topographic features which increase eddy kinetic energy in the model, several additional ridge structures are also sources of increased eddy kinetic energy. The larger background level could be attributed to instrument noise, as well as to eddy variability unresolved by the model.

While eddy kinetic energy is an indicator of heightened eddy variability, mean kinetic energy in stream coordinates (shown in Figure 4.21) is an indicator of the strength of the mean flow. Mean kinetic energy along streamlines in the numerical model output has a background level very close to zero, but peaks enormously near the three major topographic features, at Drake Passage, Kerguelen Island, and Campbell 

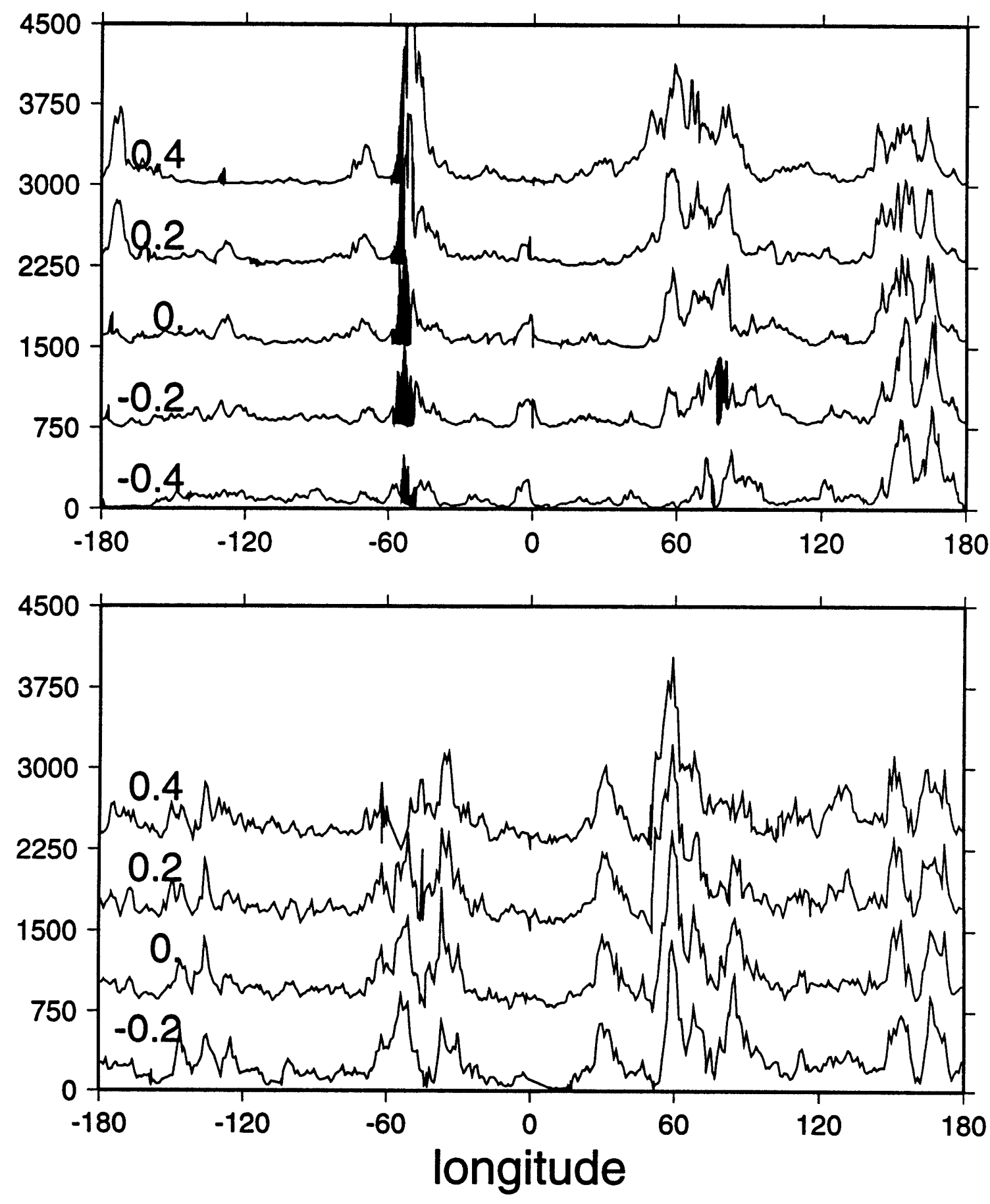

Figure 4.20: Eddy kinetic energy along surface height contours. Upper panel shows energy for the quarter-degree Semtner-Chervin model output in $\mathrm{cm}^{2} \mathrm{~s}^{-2}$, along height contours corresponding to $-0.4,-0.2,0 ., 0.2$, and 0.4 meters. Lower panel shows eddy energy along height contours in Geosat altimeter data, for contours $-0.2,0 ., 0.2$, and 0.4 meters. Error estimates obtained from the objective mapping procedure for the altimeter measurements average $50 \mathrm{~cm}^{2} \mathrm{~s}^{-2}$. 

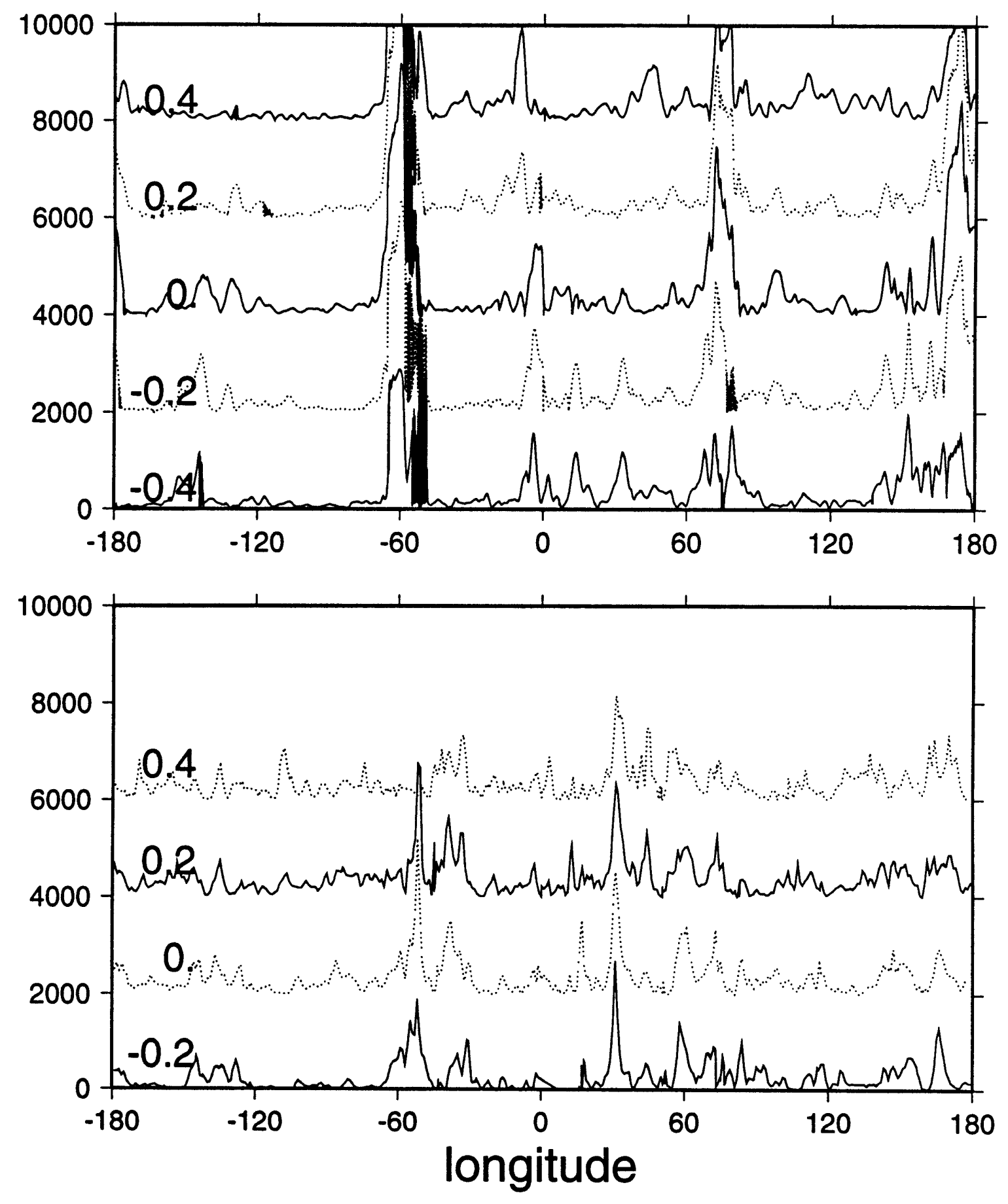

Figure 4.21: Mean kinetic energy along surface height contours. Upper panel shows energy for the quarter-degree Semtner-Chervin model output in $\mathrm{cm}^{2} \mathrm{~s}^{-2}$, along height contours corresponding to $-0.4,-0.2,0 ., 0.2$, and 0.4 meters. Lower panel shows mean energy along height contours in Geosat altimeter data, for contours -0.2, 0., 0.2, and 0.4 meters. Average error is $160 \mathrm{~cm}^{2} \mathrm{~s}^{-2}$. 
Plateau, where streamlines compress together akin to a western boundary current. Model mean kinetic energy levels also fluctuate in the mid-Atlantic and mid-Pacific Oceans. In the altimeter data, mean kinetic energy rises above its background level in the same locations. However, the altimeter results show less dramatic variations in energy levels; mean kinetic energy does not rise as high at major topographic features nor does it reduce as smoothly to low background levels in mid-ocean. Assuming that kinetic energy in the Southern Ocean is largely associated with topographically generated stationary and transient features, the observed energy differences may indicate that many more than three topographic features significantly influence ACC dynamics.

Thus, although the model appears to conform to Wang's [1993] results in showing that only large ridges control the flow, the real ocean does not. Since Wang's results rely on the ACC being confined to a narrow channel, this may occur because subsurface topographic features define deep ocean channels which are better able to function in the real ocean than in the model to create boundary currents where friction can act. Alternatively, it may indicate that instability processes which do not rely on frictional effects at topographic boundaries are active in the real ocean but not in the model.

The fact that only topographic features which breach the surface are sources of form drag and heightened eddy kinetic energy in the model also suggests that communication between the deep ocean and the surface is greater in the real ocean than in the model so that deeper topographic features have a greater impact on ocean circulation. This difference may explain why the model transport is $50 \%$ larger than the observed Southern Ocean transport. The greater impact of topography in the observed ocean relative to the model might be attributed to one of several related factors. First, it may mean that the model has too much horizontal diffusion of momentum and tracers and not enough along-isopycnal diffusion, so that momentum transfer along isopycnals is inhibited. Second, it may simply be a manifestation of 
small vertical velocities in the model related to coarse vertical resolution or unrealistic deep density fields. Third, it may indicate that the model run is not sufficiently long to spin up higher-order baroclinic modes. The period of an ocean wave is related to its frequency:

$$
T=\frac{2 \pi}{\sigma}
$$

The frequency for a Rossby wave is:

$$
\sigma=\frac{\beta k}{k^{2}+l^{2}+\frac{n^{2} \pi^{2}}{L_{R}^{2}}}
$$

where $k$ and $l$ are the wave numbers in the $x$ and $y$ directions, respectively, and $L_{R}$ is the Rossby radius, and $n$ identifies the baroclinic mode [Pedlosky, 1987]. For a barotropic wave, the Rossby radius is $L_{R}=\frac{\sqrt{g D}}{f}$ or about $2000 \mathrm{~km}$, so that for the lowest wavenumber, $T$ is about 12 days. In contrast, the Rossby radius of the first baroclinic mode is about $40 \mathrm{~km}$, resulting in a period $T$ of 140 years. The SemtnerChervin model was run for a total of 33.5 years after initialization with the observed vertical structure from Levitus. This time period should be sufficient to fully spin-up the barotropic mode, but inadequate to develop the model's baroclinic modes. Thus the barotropic mode may be disproportionately important in the results, while the baroclinic modes are not fully expressed.

\subsection{Surface Reynolds Stresses}

If surface kinetic energy offers a good comparison of overall oceanic variability in the model and altimeter, surface Reynolds stresses provide a means to thoroughly assess one term in the along-stream momentum balance. Although the hypotheses of Marshall et al. [1993] emphasized the importance of transient eddies in fluxing momentum across the ACC, this analysis of primitive equation model output has indicated that horizontal viscosity plays a more significant role than horizontal eddy 
momentum divergences through most of the water column in the numerical model. As horizontal viscosity is a parameterization of sub-grid scale processes, that could mean that the model Reynolds stress term will underestimate real physical processes, but such a problem is not evident in this study. In situ estimates of Reynolds stresses in the Southern Ocean have been limited to a few locations [Johnson and Bryden, 1989], but the Geosat altimeter provided a simple means to assess surface transient Reynolds stress terms throughout the entire Southern Ocean [eg. Morrow et al., 1992; Johnson et al., 1992].

To reduce numerical inaccuracies, Reynolds stresses rather than the divergences which appear in the momentum equations, will be considered. In stream coordinates, the cross-stream Reynolds stress term provides a measure of the transient momentum flux across the stream. It is derived from the terms in the velocity covariance matrix:

$$
\overline{u_{s}^{\prime} v_{s}^{\prime}}=\overline{u^{\prime} v^{\prime}}\left(\cos ^{2} \theta-\sin ^{2} \theta\right)+\left(\overline{v^{\prime} v^{\prime}}-\overline{u^{\prime} u^{\prime}}\right) \cos \theta \sin \theta
$$

Following along a streamline, the Reynolds stress terms (Figure 4.22) show similar patterns. Both the numerical model and the altimeter measurements indicate heightened Reynolds stress amplitudes in the locations corresponding to large topographic features, where energy levels were also elevated. However, the altimeter indicates a less distinct separation between two different domains. Neither shows a major identifiable trend with longitude or cross-stream distance, although Reynolds stress variability does decrease with decreasing sea surface height near $60^{\circ} \mathrm{E}$ in the numerical model output; this may occur because only the northern portion of the flow is steered over the Crozet Plateau in this region.

Figure 4.23 shows along-stream averaged Reynolds stresses for the numerical model and Geosat data. Altimeter error ranges, indicated by dotted lines, account for errors in objective mapping, but do not include errors in the rotation angle $\theta$, so they may tend to underestimate the overall errors. Nonetheless, the overall comparison is 

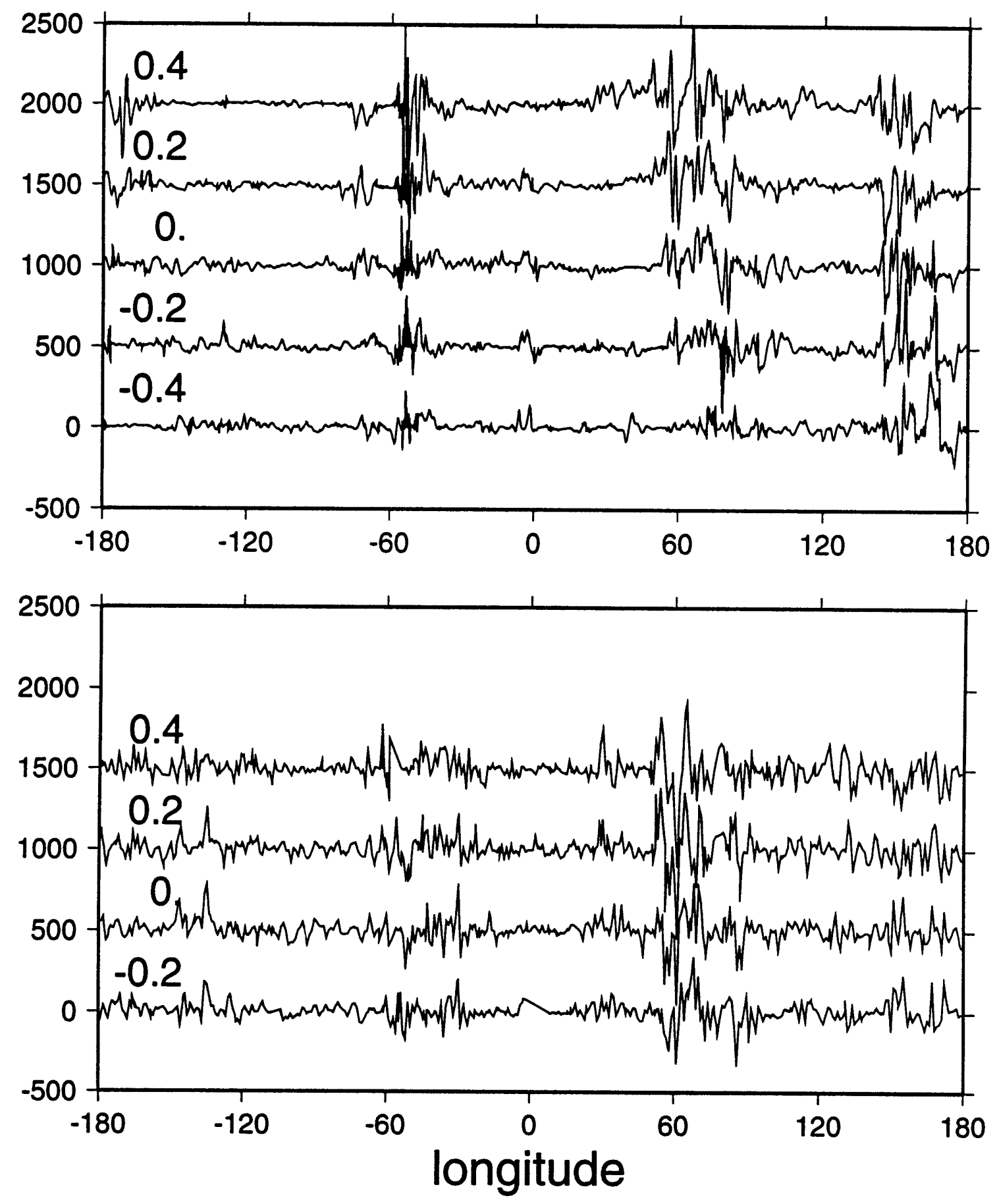

Figure 4.22: Transient cross-stream Reynolds stresses along surface height contours. Upper panel shows Reynolds stress for the quarter-degree Semtner-Chervin model output in $\mathrm{cm}^{2} \mathrm{~s}^{-2}$, along height contours corresponding to $-0.4,-0.2,0 ., 0.2$, and 0.4 meters. Lower panel shows Reynolds stress along height contours in Geosat altimeter data, for contours $-0.2,0 ., 0.2$, and 0.4 meters. Error estimates obtained from the objective mapping procedure for the altimeter measurements average $20 \mathrm{~cm}^{2} \mathrm{~s}^{-2}$. 

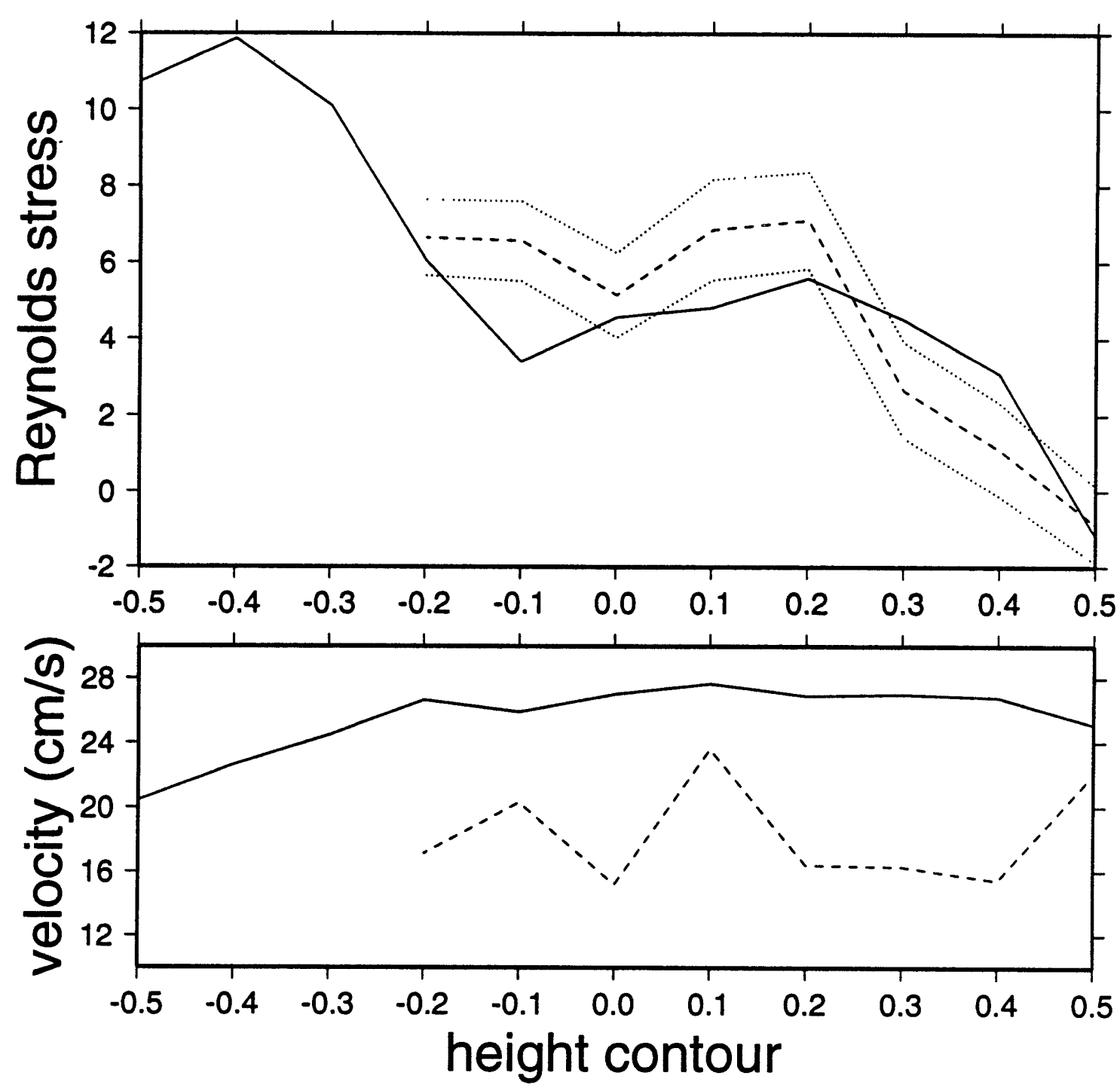

Figure 4.23: (Top) Transient cross-stream Reynolds stresses averaged along surface height contours in $\mathrm{cm}^{2} \mathrm{~s}^{-2}$. Solid line shows Reynolds stress terms for the quarterdegree Semtner-Chervin model output. Dashed line shows Reynolds stress in Geosat altimeter data, with the one sigma error range indicated by dotted lines. Sea surface height levels are indicated in meters; the 0 line corresponds roughly to the separation between the SAF and the PF at the center of the ACC. (Bottom) Along-stream surface velocity averaged along surface height contours in $\mathrm{cm} \mathrm{s}^{-1}$ for (solid) SemtnerChervin model and (dashed) Geosat altimeter. The velocity maxima corresponding to the SAF and PF occur at $0.1 \mathrm{~m}$ and $-0.1 \mathrm{~m}$ height for the altimeter, and at $0.1 \mathrm{~m}$ and $-0.2 \mathrm{~m}$ height for the numerical model. 
extremely close, both in magnitude and trend, suggesting that the surface transient eddy fluxes are captured in a consistent way by numerical model and by this analysis of altimeter data. Reynolds stresses are positive meaning that zonal momentum is fluxed equatorward across the circumpolar current. The terms decrease to the north, with increasing sea surface height, suggesting that across most of the ACC, transient eddy terms act to accelerate the flow, as suggested by the analysis of Morrow et al. [1992]. However, in the narrow region which roughly separates the SAF and the PF, Reynolds stress terms increase, suggesting that transient eddies decelerate the mean flow through this region. In a coarser resolution analysis, Wilkin and Morrow [1994] found similar results in the Agulhas retroflection region: in Geosat data combined with a mean surface velocity from atlas data and in the half-degree Semtner-Chervin model, eddies tend to drive the mean flow within the core of the current, but decelerate it in regions far from the ACC axis. These results are exactly opposite to the findings of Ivchenko et al. [1994], based on FRAM, which showed that in stream coordinates transient eddies tend to slow the ACC.

\subsection{Summary}

This combination of numerical model output and altimeter data used for this investigation has elucidated several key features of the Southern Ocean momentum balance.

The momentum balance has been examined by averaging along zonal coordinates, by averaging along stream coordinates, and by examining the along-stream variations of the momentum balance. Consistent with previous studies, the vertically averaged balance shows that form drag balances wind stress, both in zonal coordinates and in stream coordinates. In zonally averaged coordinates, the residual difference between wind stress and form drag is predominantly balanced by mean horizontal momentum stress divergences while biharmonic friction and eddy flux divergences 
play a less important role. Averaging along streamlines diminishes the role of the mean eddy flux divergences, since the cross-stream mean geostrophic velocity is zero. Instead biharmonic friction is the largest term balancing the form drag divergence, while the mean equatorward momentum flux divergence continues to contribute to the balance, though this is largely an artifact of transforming coordinates of model output at discretized grid points. The mean vertical momentum flux divergence tends to accelerate the flow; bottom friction and transient eddy flux divergences are less significant. Individual levels of the ocean model have similar balances: the surface Ekman layer counters wind forcing in the upper ocean, and the residual difference is balanced by a combination of mean and transient eddy flux divergences and biharmonic friction, both in zonally averaged and along-stream coordinates. At mid-depth, wind stress does not directly act on the ocean; in the zonally averaged momentum equations, the vertical divergence of form drag is balanced by the mean horizontal momentum flux divergence, while averaged along streamlines, horizontal eddy viscosity balances form drag. Horizontal viscosity is a parameterization of sub-grid scale processes; if the magnitude of the terms in these results is representative of real ocean processes, then either the model resolution is inadequate or a more physically justified representation of eddy viscosity should be found. The magnitude of horizontal viscosity, particularly at depth, suggests that the dominant scales of motion are smaller than the grid spacing or, alternatively, that the particular parameterization used in the model is inadequate meaning that an isopycnal formulation should be adopted. Both explanations indicate that the parameterization of sub-grid scale processes is extremely important. Alternatively the horizontal viscosity coefficient in the model may simply be unrealistically large.

At all depths, the along-stream momentum balance shows substantial variability associated with three large topographic features: Drake Passage, Kerguelen Island, and Campbell Plateau. At each of these, land intersects the path of the ACC, and essentially all form drag is due to these three features. Despite the substan- 
tial variability, the relative importance of terms in the momentum balance does not change substantially between regions associated with topographic barriers and quiescent mid-basin regions. The major differences appear to be in the kinetic energy level rather than the structure of the dynamical balance. Given this observation, eddy kinetic energy has been examined along streamlines in the numerical model and in the altimeter measurements.

Both model and data feature narrow meandering jets which comprise the ACC, and energy levels are greatest along the jet axes. Overall the altimeter data indicate twice as much eddy kinetic energy as the model output predicts. This difference, coupled with the surprising importance of horizontal viscosity in the model, suggests that the model resolution may not be sufficient to capture relevant eddy processes. Along surface streamlines, model eddy kinetic energy shows three substantial peaks, corresponding to the three major topographic features, all of which extend through the entire water column. In the altimeter measurements, eddy kinetic energy peaks at these same locations, but also at many smaller topographic features, indicating that minor ridges may also be sources of form drag. This suggests that communication between depth and the surface may be better in the real ocean than in the model, either due to the model's excessive viscosity, inadequate spin-up, or other baroclinic processes which may inhibit vertical velocity.

Finally, transient Reynolds stress terms play a small role in the the overall surface momentum balance; nonetheless, altimeter and model measurements closely agree. Reynolds stresses are generally positive, indicating that eddies flux zonal momentum equatorward. The terms decrease with increasing latitude, suggesting that transient eddies tend to accelerate the mean flow, except in the region between the major fronts which comprise the ACC.

The close match of altimeter and model Reynolds stress terms, the resemblances in jet structures, and the order of magnitude agreement in energy levels together suggest that the numerical model captures upper ocean physics reasonably 
well. Given the basic similarities between model and data, the model physics is not an implausible representation of the Southern Ocean; discrepancies between model and measurements offer additional insight into the critical dynamics governing the ACC, and suggest that the real ocean experiences much more substantial vertical communication than the model.

\subsection{Appendix: Accounting for non-linearities in the equation of state.}

Although a reasonable approximation of the pressure gradient term may be obtained using mean temperature and mean salinity, the estimate is improved by taking advantage of the measured variability. (Similar approaches are used to ameliorate wind stress estimates when only monthly mean and standard deviations are available [Wright and Thompson, 1983].)

The model equation of state is:

$$
\rho=\frac{1}{A+\frac{p_{1}}{p_{2}}}
$$

where

$$
p_{1}=a_{1}+a_{2} t+a_{3} t^{2}+a_{4} s+a_{5} t s
$$

and

$$
p_{2}=b_{1}+b_{2} z+b_{3} s+b_{4} t+b_{5} t^{2}
$$

where $z$ is defined as depth, $t$ is temperature, and $s$ is salinity.

Time averaging and rewriting (4.23):

$$
\begin{aligned}
\bar{\rho} & =\frac{\overline{p_{2}}}{A \overline{p_{2}}+\overline{p_{1}}} \overline{\left(\frac{1+\frac{p_{2}^{\prime}}{\overline{p_{2}}}}{\left.1+\frac{A p_{2}^{\prime}+p_{1}^{\prime}}{A \overline{p_{2}}+\overline{p_{1}}}\right)}\right.} \\
& \approx \frac{\overline{p_{2}}}{A \overline{p_{2}}+\overline{p_{1}}}\left(1+\frac{p_{2}^{\prime}}{\overline{p_{2}}}\right)\left(1-\frac{A p_{2}^{\prime}+p_{1}^{\prime}}{A \overline{p_{2}}+\overline{p_{1}}}+\left(\frac{A p_{2}^{\prime}+p_{1}^{\prime}}{A \overline{p_{2}}+\overline{p_{1}}}\right)^{2}\right)
\end{aligned}
$$




$$
\begin{aligned}
\approx & \tilde{\rho}\left(1+\frac{b_{5} \sigma_{t}^{2}}{\overline{p_{2}}}-\frac{\tilde{\rho}}{\overline{p_{2}}}\left(A b_{5} \sigma_{t}^{2}+a_{3} \sigma_{t}^{2}\right)\right. \\
& -\frac{\tilde{\rho}}{{\overline{p_{2}}}^{2}}\left(A b_{3}^{2} \sigma_{s}^{2}+A b_{4} \sigma_{t}^{2}+b_{3} a_{4} \sigma_{s}^{2}+a_{2} b_{4} \sigma_{t}^{2}\right) \\
& \left.+\frac{\tilde{\rho}^{2}}{{\overline{p_{2}}}^{2}}\left(A^{2} b_{3}^{2} \sigma_{s}^{2}+A^{2} b_{4}^{2} \sigma_{t}^{2}+2 A b_{3} a_{4} \sigma_{s}^{2}+2 A a_{2} b_{4} \sigma_{t}^{2}+a_{2}^{2} \sigma_{t}^{2}+a_{4}^{2} \sigma_{s}^{2}+a_{5}^{2} \sigma_{t}^{2} \sigma_{s}^{2}\right)\right)
\end{aligned}
$$

where $\overline{p_{i}}$ are short hands for $p_{i}(\bar{t}, \bar{s})$ and $\tilde{\rho}$ is $\rho(\bar{s}, \bar{t})$. Temperature-salinity covariance terms have not been included in this expansion because the Semtner-Chervin model output does not include this information. Since $p_{i}^{\prime}<<\overline{p_{i}}$, this approximation is generally valid and has been implemented. The resulting density fields differ slightly from the original estimate, obtained using $\rho(\bar{s}, \bar{t})$, but are virtually the same as the estimate obtained by correcting $p_{i}$ by using $\sigma_{t}$ to adjust the $t^{2}$ terms. Not surprisingly the correction has the most significant impact near regions of large temperature gradients where the standard deviation may be greatest. 


\section{Chapter 5}

\section{Potential Vorticity}

\subsection{Introduction}

Basic conceptualizations of ocean circulation have emphasized the dynamics of flatbottomed rectangular basins. A broad, almost imperceptibly slow interior flow is controlled by Sverdrup dynamics:

$$
\beta V=\frac{\operatorname{curl} \tau}{\rho_{0}}
$$

where $\beta$ is the meridional derivative of the Coriolis parameter $f, V$ is the total meridional transport, $\tau$ is the wind stress, and $\rho_{0}$ is density. Thus the vorticity input by the wind ( $\operatorname{curl} \tau$ ) is balanced by changes in planetary vorticity $(f)$. The large scale flow is returned in a more rapid western boundary current, where friction may remove the vorticity input by the wind stress curl, or nonlinear processes may advect vorticity away from the mean jet to regions where it is more directly dissipated [Stommel, 1948; Munk, 1950; Fofonoff, 1954].

Because the Southern Ocean is a continuous channel without well-defined meridional boundaries, it does not easily conform to the traditional rectangular basin vorticity balances. Although the wind over the Southern Ocean is extremely strong, no continental boundaries exist to remove the vorticity input by the wind stress curl. 
Nonetheless, the ACC is not continuously accelerating, and Sverdrup dynamics do not drive a uniform and perpetual southward flow. As a result, many studies have assumed that ACC dynamics are non-Sverdrupian like atmospheric jet stream dynamics, that vorticity is dissipated as it enters through the action of nonlinear and frictional terms. Munk and Palmén [1951] suggested that topographic form stress, drag associated with the pressure gradient across topographic ridges, was the principal method to balance the wind forcing in the momentum balance; by analogy this would suggest that bottom pressure torques should balance the wind stress curl in the vorticity equation. Gill [1968] explored the importance of lateral friction in the vorticity equation, but found that bottom and side friction coefficients would have to be unrealistically large. Other work has speculated that Sverdrup dynamics may apply in spite of there being no true western boundary: Stommel [1957] suggested that the Southern Ocean spirals southward over most of its length, driven by the wind stress curl represented by Sverdrup theory, and returns northward in two narrow currents located near Campbell Plateau and just east of Drake Passage. Baker [1982] used wind measurements and Drake Passage transport estimates to confirm that southward transport in the ACC is consistent with a Sverdrup balance.

More recent work using numerical model output has specifically examined the vorticity budget in the primitive equation Fine Resolution Antarctic Model (FRAM): Wells and de Cuevas [1994] found that the curl of the wind stress was predominantly balanced by the bottom pressure torque which acts almost entirely in the region around and just downstream of Drake Passage. The $\beta$-effect plays an important role in the local vorticity balance by compensating fluctuations in the bottom pressure torque. Integrating along the path of the ACC indicated that on larger scales the ACC changes latitude to balance the vorticity due to wind stress curl. Because the bottom pressure torque and nonlinear advective terms are large, however, they found that the Sverdrup balance does not hold explicity except in relatively small eastern portions of the ocean basins, north of the main ACC axes. Their results echo the 
findings of momentum balance studies of FRAM model output which showed that topographic form drag provided the dominant balance for the surface wind forcing.

While the momentum balance is locally geostrophic, in the vorticity equations, the dominant geostrophic relation has been eliminated, so that the underlying forcing effects are more easily identified. The vorticity equations, however, are particularly limiting for investigating ocean dynamics from relatively sparse data because of the errors associated with computing the additional horizontal derivatives needed to convert velocity to vorticity. Thus, for most observational purposes, the vorticity equations do not offer additional insight.

Potential vorticity, which arises in the quasi-geostrophic vorticity equations, offers two advantages over vorticity itself. First, in isopycnal coordinates, it may be computed directly from the vertical density structure, so that it is easily calculated from hydrographic data. Second, in an unforced system, it is conserved along geostrophic streamlines, which makes it a valuable indicator. If the quasi-geostrophic approximation is appropriate, potential vorticity will be constant along streamlines if there is no forcing or if forcing is dissipated in the same geographic locations where it acts. Alternatively, if forcing and dissipation do not locally balance, then potential vorticity will undergo dramatic changes in value; the geographic locations of these changes may be useful indicators of the leading mechanisms controlling the dynamics.

Several recent studies have taken advantage of potential vorticity to probe Southern Ocean dynamics. Marshall et al. [1993] applied zero eddy flux constraints and an assumption that no forcing occurs beneath the surface layer to argue that potential vorticity is homogenized on isopycnal surfaces. If this result fully applied in the ACC itself, as they suggested, then potential vorticity would be a linear function of potential density; however, as Marshall et al. noted, the data indicate large-scale variations in potential vorticity which could suggest the presence of forcing at depth.

Marshall [1994b] predicted the vertical structure and path of the ACC by assuming that potential vorticity is conserved along streamlines, imposing the ad- 
ditional constraints that density be constant along streamlines on the sea floor and that the linear vorticity balance apply. Using realistic bathymetry, but applying no surface wind stress curl, he found a solution set of streamlines which are substantially steered by topography, particularly around the Kerguelen Plateau.

Rather than assuming potential vorticity is conserved on isopycnal streamlines, this study will examine the variation of potential vorticity along streamlines in order to probe the forcing and dissipation of the ACC. If form stress is the critical process balancing wind stress in the Southern Ocean, then where is it localized? What size ridges are critical for the process? Are numerical models capturing this result reasonably?

Potential vorticity will be considered in two different ways. First section 5.2 will examine flow along potential density surfaces in numerical model output. Second, in order to compare model output with sea surface height measurements from the Geosat altimeter, in section 5.3 two proxies for potential vorticity on surface streamlines will be explored; both proxies assume an equivalent barotropic vertical structure in the ACC. The one developed for this study fixes no level of no motion, while Marshall's [1994a] formulation assumes slightly different boundary conditions which require establishing a fixed level of no motion near the bottom. The two approaches are compared in the appendix. The results of this study are summarized in section 5.4

\subsection{Potential Vorticity on Isopycnals}

\subsubsection{Defining Potential Vorticity}

The Semtner-Chervin quarter-degree primitive equation model was run for $3 \frac{3}{4}$ years after spin-up. Its time-averaged output offers a near eddy-resolving data set with which to investigate potential vorticity variations along the path of the ACC. 
On a surface of constant potential density, the horizontal equation of motion may be written:

$$
\frac{\partial \mathbf{u}}{\partial t}+f \mathbf{k} \times \mathbf{u}+(\mathbf{u} \cdot \nabla) \mathbf{u}+\nabla M=\mu \frac{\partial^{2} \mathbf{u}}{\partial z^{2}}+A_{m} \nabla^{4} \mathbf{u}
$$

with the continuity equation:

$$
\nabla \cdot\left(\mathbf{u} \frac{\partial z}{\partial \sigma}\right)=-\frac{\partial}{\partial z_{c}}\left(w \frac{\partial z}{\partial \sigma}\right) \approx 0
$$

where $\nabla$ is the horizontal gradient operator defined along an isopycnal surface, $u$ is the along isopycnal surface velocity, $z_{c}$ is oriented in the cross-isopycnal direction, and $w$ is the cross-isopycnal velocity and is normally imagined to be zero, so that the left side of the continuity equation in isopycnal coordinates is also zero. Since the SemtnerChervin code is conceived on levels rather than isopycnal layers, mass is not conserved on layers and this continuity equation does not hold exactly. Its deviation from zero indicates the cross-isopycnal velocity in the model which may be largely associated with surface Ekman pumping. $M$ is a streamfunction, in essence representing the pressure term transformed into isopycnal coordinates. Although McDougall [1989] asserts that no correct streamfunction exists for potential density surfaces, Zhang and Hogg [1992] showed that the geostrophic streamlines on potential density surfaces may be approximated by the Montgomery streamfunction on a surface of constant specific volume anomaly ${ }^{1}, \delta$, so that:

$$
M=\delta(z)(p(z)-\bar{p})-\int^{p} \delta(z) d p
$$

where $z$ represents the depth of the isopycnal surface and varies spatially, and

$$
\delta(z)=\frac{1}{\rho(z)}-\frac{1}{\rho_{o}(z)} .
$$

The mean pressure, $\bar{p}$, is subtracted in order to minimize errors associated with the approximation. The addition of a free surface, $\eta$, means that $M$ may be written:

$$
M=\delta \eta g \rho_{s}+\delta \int_{z}^{0} g \rho d z^{\prime}-\delta \bar{p}-\int^{p} \delta d p
$$

\footnotetext{
${ }^{1}$ The Bernoulli function, $B=p+\rho g z$, is often substituted for $M \rho_{0}$. It is correct for constant density surfaces, but not for constant potential density surfaces.
} 
The Montgomery potential for the Semtner-Chervin model output, divided by $g \rho_{0}$ is contoured in Figure 5.1. $M$ closely resembles sea surface height, represented in Figure 5.2, showing a similar zonal flow structure with intensified western boundary current jets. The smaller overall height difference for $M$ compared with the sea surface height illustrates the degree to which velocities along the potential density surface are attenuated relative to the surface.

Taking the curl of the time-averaged momentum equation (5.2) and substituting in from the continuity equation (5.3), produces an approximate expression for conservation of potential vorticity along isopycnals:

$$
\frac{\zeta_{f}-\zeta_{i}}{\Delta t}+\frac{\partial z}{\partial \sigma} J(\bar{\psi}, \bar{Q})+\frac{\partial z}{\partial \sigma} \overline{\mathbf{u}}_{a g} \cdot \nabla \bar{Q}+\overline{u^{\prime} \nabla \zeta^{\prime}}+\bar{Q} \nabla \cdot\left(\overline{\mathbf{u}} \frac{\partial z}{\partial \sigma}\right)=\mu \frac{\partial^{2} \bar{\zeta}}{\partial z^{2}}+A_{m} \nabla^{2} \bar{\zeta}
$$

with potential vorticity, $Q$, defined as

$$
Q=(f+\zeta)\left(\frac{\partial z}{\partial \sigma}\right)^{-1}
$$

$J$ is the Jacobian operator, overbars represent time averages and primes represent deviations from those averages, $\zeta$ is the relative vorticity, $v_{x}-u_{y}, \bar{\psi}$ is the mean streamfunction within the isopycnal layer, and $\overline{\mathbf{u}}_{a g}$ is the mean ageostrophic velocity which, in the case of large Ekman transports, could account for a substantial portion of the vorticity balance. Thus in $\left(5.7, \frac{\zeta_{f}-\zeta_{i}}{\Delta t}\right.$ represents the change in relative vorticity over the total time interval considered, $\Delta t ; \frac{\partial z}{\partial \sigma} J(\bar{\psi}, \bar{Q})$ indicates the change in mean potential vorticity along a mean geostrophic streamline, $\bar{\psi} ; \frac{\partial z}{\partial \sigma} \overline{\mathbf{u}}_{a g} \cdot \nabla \bar{Q}$ is the advection of mean potential vorticity due to the ageostrophic component of velocity; $\overline{u^{\prime} \nabla \zeta^{\prime}}$ represents the advection of transient potential vorticity; and $\bar{Q} \nabla \cdot\left(\overline{\mathbf{u}} \frac{\partial z}{\partial \sigma}\right)$ is the stretching term due to changes in isopycnal layer thickness. Potential vorticity is transmitted vertically by the vertical viscosity term $\mu \frac{\partial^{2} \bar{\zeta}}{\partial z^{2}}$ and redistributed horizontally by $A_{m} \nabla^{4} \bar{\zeta}$ The density structure, $\frac{\partial z}{\partial \sigma}$, is assumed to be constant with time to leading order. $Q$ for the mean Semtner-Chervin model output on the $\sigma_{1}=32.3$ surface is shown in Figure 5.3. 


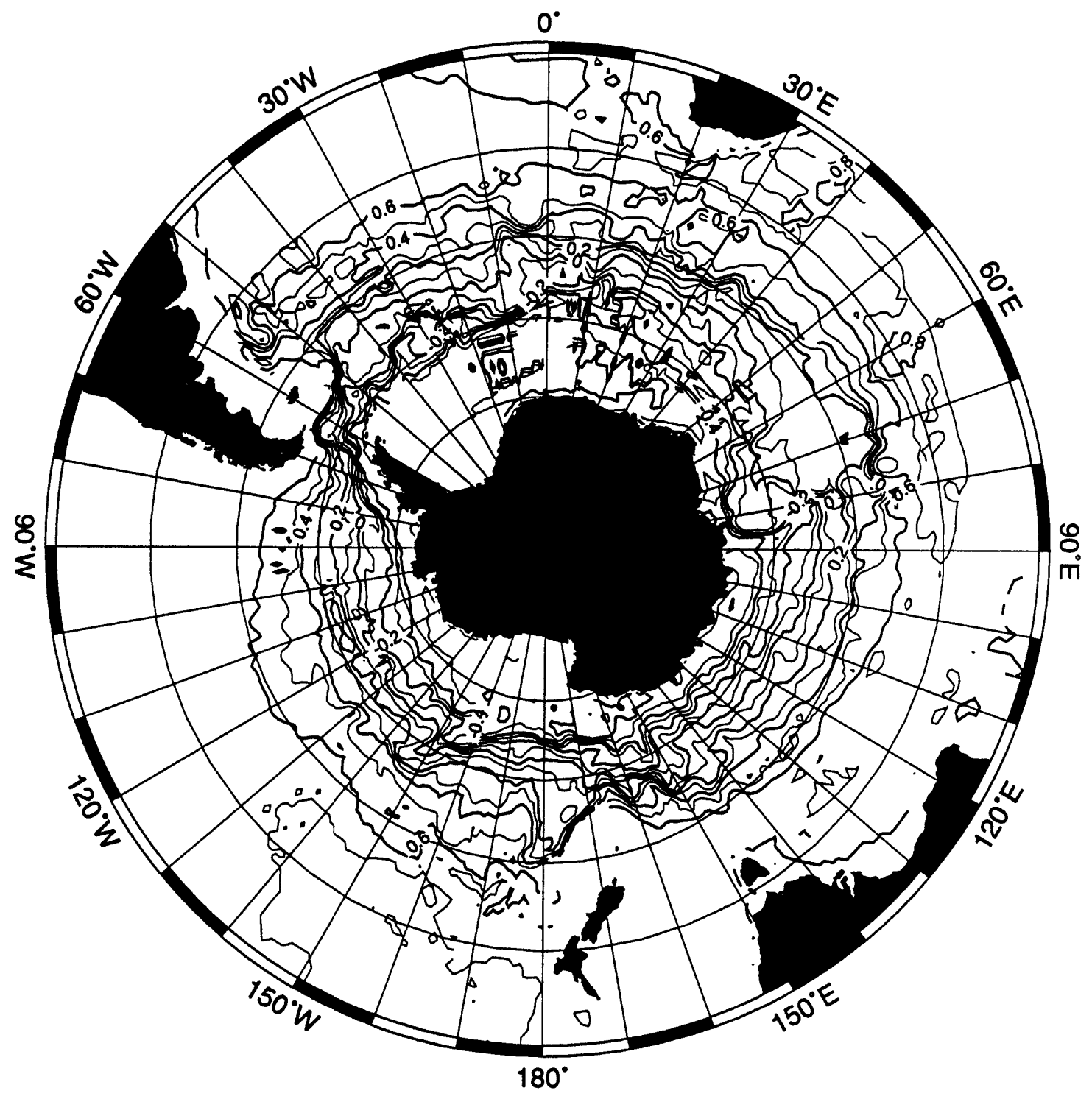

Figure 5.1: Contours of constant Montgomery streamfunction divided by $g \rho_{0}$ on the potential density surface, $\sigma_{1}=32.3$, determined from the Semtner-Chervin quarterdegree numerical model. The contour interval is $0.1 \mathrm{~m}$, and the $0 \mathrm{~m}$ height contour is fixed for simple comparison with sea surface height shown in Figure 5.2 


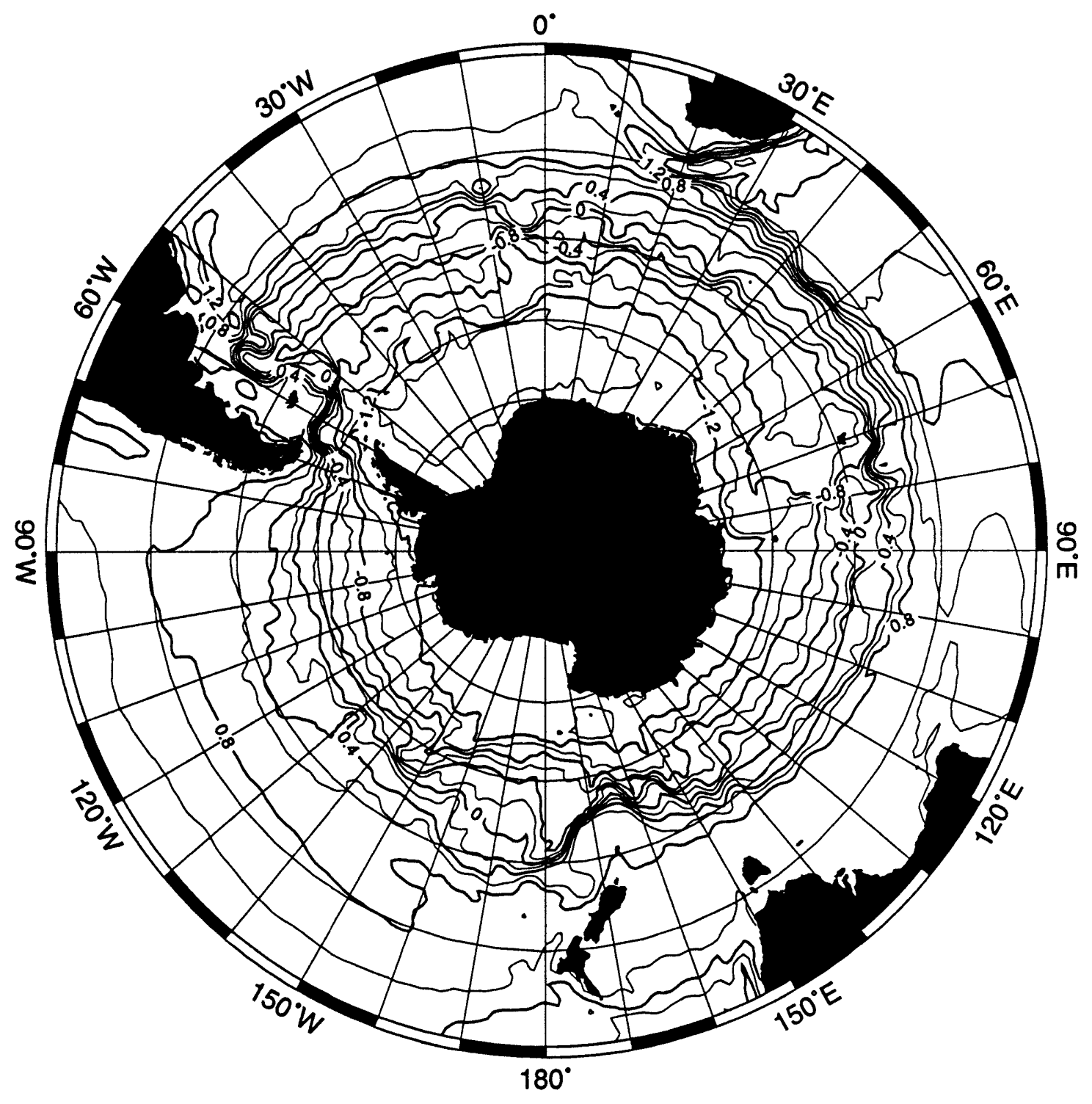

Figure 5.2: Contours of sea surface height from the Semtner-Chervin quarter-degree numerical model. The contour interval is $0.2 \mathrm{~m}$. 


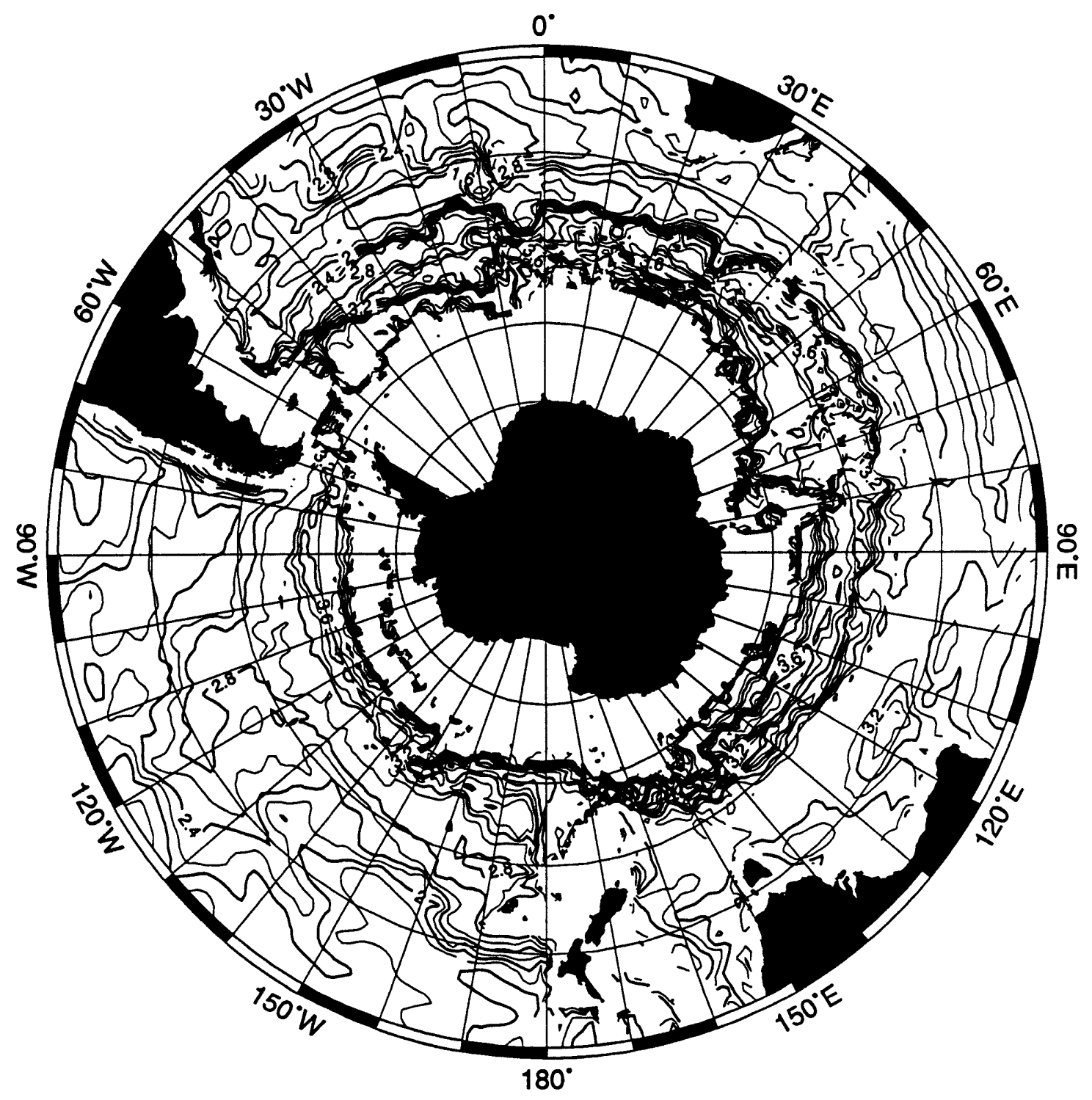

Figure 5.3: Potential vorticity, $(f+\zeta) \frac{\partial \sigma}{\partial z}$, (in $\left.\mathrm{kg} \mathrm{s}^{-1} \mathrm{~m}^{-4} \times 10^{8}\right)$ on the $\sigma_{1}$ surface 32.3 determined from mean quarter-degree Semtner-Chervin model output. The $\sigma_{1}$ surface outcrops at the southern limit of the domain and is several kilometers deep at the northern limit. 
In the case where the system is in steady state and ageostrophic effects, crossisopycnal velocities, surface forcing, and horizontal and vertical viscosity are negligible then potential vorticity is conserved along streamlines, so that $J(\psi, Q)=0$. This assumption has been used extensively in theoretical studies.

\subsubsection{Forcing Terms on Streamlines}

If potential vorticity does vary along streamlines, then its variations should be explained by the forcing terms in the vorticity equations. To compare potential vorticity on streamlines, the vorticity equation (5.7) is projected into stream coordinates $(s, c)$, where $s$ is the along-stream direction and $c$ is the cross-stream direction. Assuming steady state and expanding the Jacobian operator produces:

$$
\begin{aligned}
& \frac{\partial \psi}{\partial c} \frac{\partial Q}{\partial s}= \\
& \quad\left(\frac{\partial z}{\partial \sigma}\right)^{-1} \mu \frac{\partial^{2} \bar{\zeta}}{\partial z^{2}}+\left(\frac{\partial z}{\partial \sigma}\right)^{-1} A_{m} \nabla^{4} \bar{\zeta}-\overline{\mathbf{u}}_{a g} \cdot \nabla \bar{Q} \\
& \quad-\left(\frac{\partial z}{\partial \sigma}\right)^{-1} \overline{u^{\prime} \nabla \zeta^{\prime}}-\left(\frac{\partial z}{\partial \sigma}\right)^{-1} \bar{Q} \nabla \cdot\left(\overline{\mathbf{u}} \frac{\partial z}{\partial \sigma}\right)
\end{aligned}
$$

since $\psi$, is 0 , by definition. The change in potential vorticity is found by integrating, so that:

$$
Q(s)-Q(0)=\int_{0}^{s} \frac{A_{m} \nabla^{4} \bar{\zeta}}{\frac{\partial z}{\partial \sigma} \frac{\partial \psi}{\partial c}} d s^{\prime}-\int_{0}^{s} \frac{\overline{\mathbf{u}}_{a g} \cdot \nabla Q}{\frac{\partial \psi}{\partial c}} d s^{\prime}+\int_{0}^{s} \frac{1}{\frac{\partial z}{\partial \sigma} \frac{\partial \psi}{\partial c} \bar{Q}} \nabla \cdot\left(\overline{\mathbf{u}} \frac{\partial z}{\partial \sigma}\right) d s^{\prime}+\ldots
$$

where $A_{m} \nabla^{4} \bar{\zeta}$ represents horizontal viscosity, $\overline{\mathbf{u}}_{a g} \cdot \nabla Q$ is the advection of potential vorticity by the ageostrophic component of velocity, and $\bar{Q} \nabla \cdot\left(\overline{\mathbf{u}} \frac{\partial \mathbf{z}}{\partial \sigma}\right)$ is the divergence of along-isopycnal velocity which is usually associated with Ekman pumping. The terms from (5.9) which are omitted in (5.10) are negligibly small. The expression $\frac{\partial z}{\partial \sigma} \frac{\partial \psi}{\partial c}$ represents the along-stream geostrophic transport. Thus the integrated form of any forcing term in the vorticity equation divided by along-stream transport is the appropriate quantity to compare to potential vorticity. 
(Extending this result illustrates that potential vorticity itself merely represents a transport weighted along-stream integral of the second and fourth terms on the left-hand side of the vorticity equation (5.7). That is:

$$
Q(s)=\int_{0}^{s}\left(\frac{\partial \psi}{\partial c}\right)^{-1}\left[\mathbf{u} \cdot \nabla\left((\zeta+f)\left(\frac{\partial z}{\partial \sigma}\right)^{-1}\right)\right] d s^{\prime}+Q(0)
$$

Thus the vorticity advection term is given a larger weight in geographic locations where the transport is smaller; in essence, $Q$ is a time integral of vorticity advection along streamlines.)

\subsubsection{Potential Vorticity on Montgomery Streamfunction}

Potential vorticity, defined by (5.8), and the forcing terms governing it, defined by (5.10), are computed along contours of constant Montgomery streamfunction on the potential density surface $\sigma_{1}=32.3$ in the Semtner-Chervin model output. Figure 5.4a shows that potential vorticity is varies by about $25 \%$ along streamlines, increasing slightly across the Pacific and decreasing in the Atlantic. Figures 5.4b and 5.5 show the relative importance of each of the components of potential vorticity. The major variations are attributed to changes in the layer thickness $\left(\frac{\partial z}{\partial \sigma}\right)$ while variations in latitude $(f)$ have only minor effects and relative vorticity is negligible. Therefore the Sverdrup balance $\left(\mathbf{U} \cdot \nabla f \approx \operatorname{curl} \tau / \rho_{\mathrm{o}}\right.$ ) commonly cited for closed basins is not the dominant process in this numerical Southern Ocean; instead, density changes along streamlines provide the major compensation for the wind forcing, so that if we integrate vertically, we predict $\int_{-H}^{0} \frac{\partial z}{\partial \sigma} \mathbf{u} \cdot \nabla\left(f \frac{\partial z}{\partial \sigma}^{-1}\right) d z=$ curl $\tau / \rho$. The ECMWF wind stress curl is positive to the north of about $50^{\circ} \mathrm{S}$. The stratification term, $\frac{\partial z}{\partial \sigma}$ is negative, while $\mathbf{u}$ is positive. Thus, in the Atlantic and Indian sectors of the Southern Ocean, where the ACC is north of $50^{\circ} \mathrm{S}$, potential vorticity tends to decrease along streamlines. Where the ACC is further south and wind stress curl is negative, potential vorticity tends to increase. 

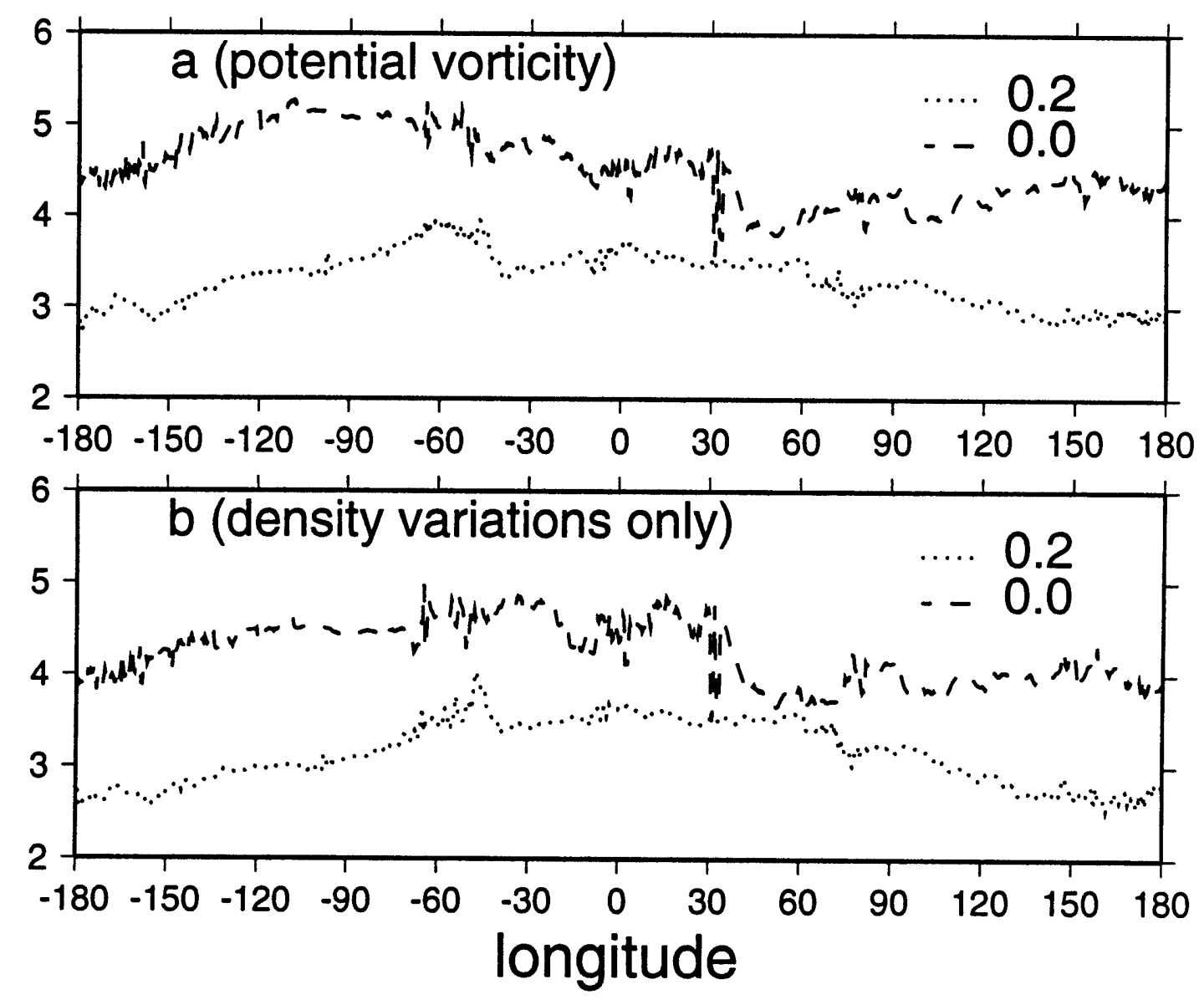

Figure 5.4: (a) Potential vorticity (in $\mathrm{kg} \mathrm{s}^{-1} \mathrm{~m}^{-4} \times 10^{8}$ ) along Montgomery streamfunction, $M$, from the Semtner-Chervin quarter-degree mean model output. Contours shown correspond to 0.2 and $0.0 \mathrm{~m}$, where the $0 \mathrm{~m}$ contour is fixed to correspond approximately to the center of the ACC. Sea surface height increases toward the equator, so that the $0.2 \mathrm{~m}$ contour is north of the $0.0 \mathrm{~m}$ contour. (b) Potential vorticity along Montgomery streamfunction, when $f+\zeta$ is fixed so that only changes in the stratification, $\frac{\partial \sigma}{\partial z}$, are assessed. 


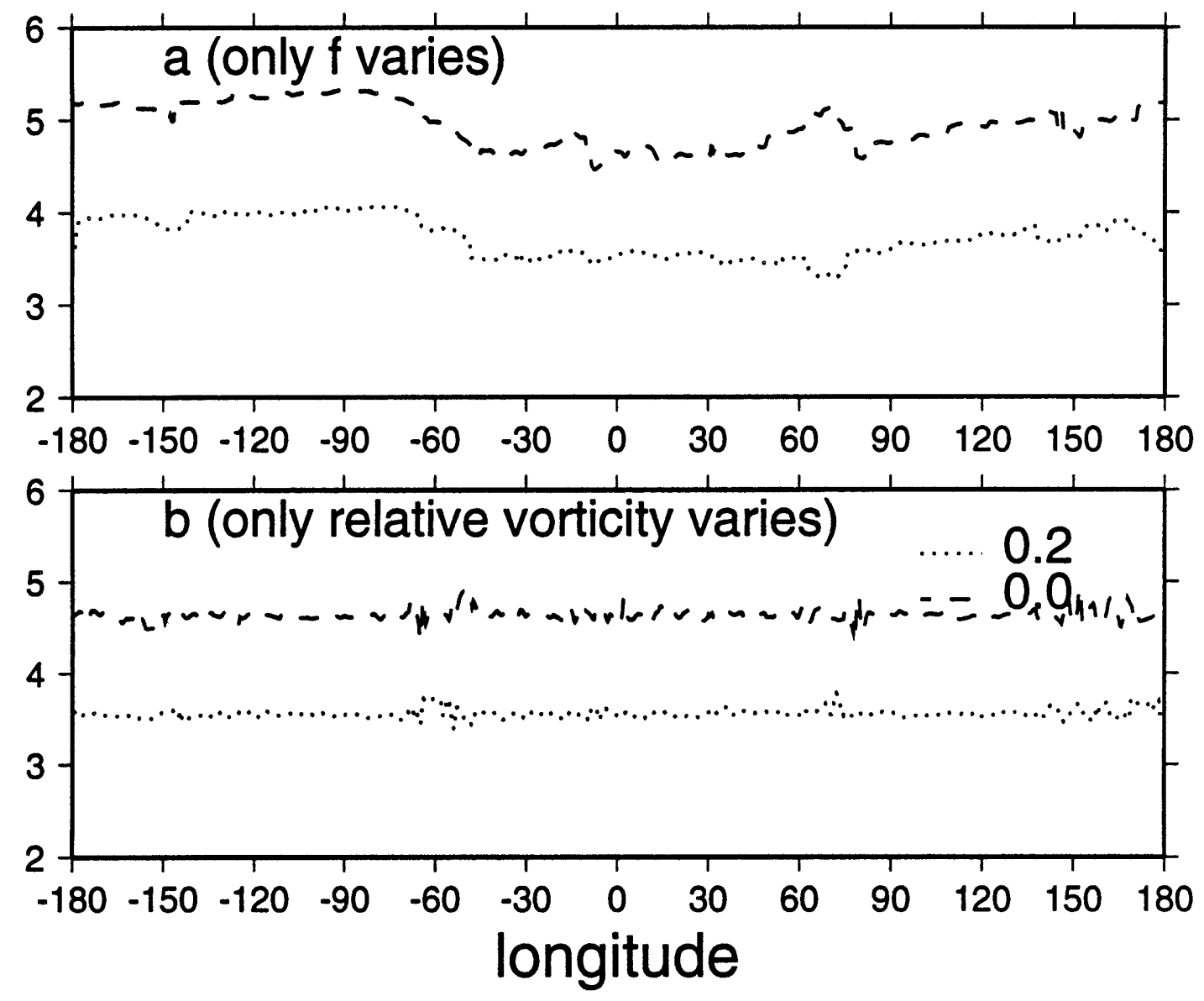

Figure 5.5: (a) Potential vorticity along Montgomery streamfunction, where only stratification and relative vorticity are held constant, so that only changes in latitude are assessed. Levels are fixed as in Figure 5.4. (b) Potential vorticity along Montgomery streamfunction, when $f$ and $\frac{\partial \sigma}{\partial z}$ are held fixed so that only the effect of relative vorticity is considered. 


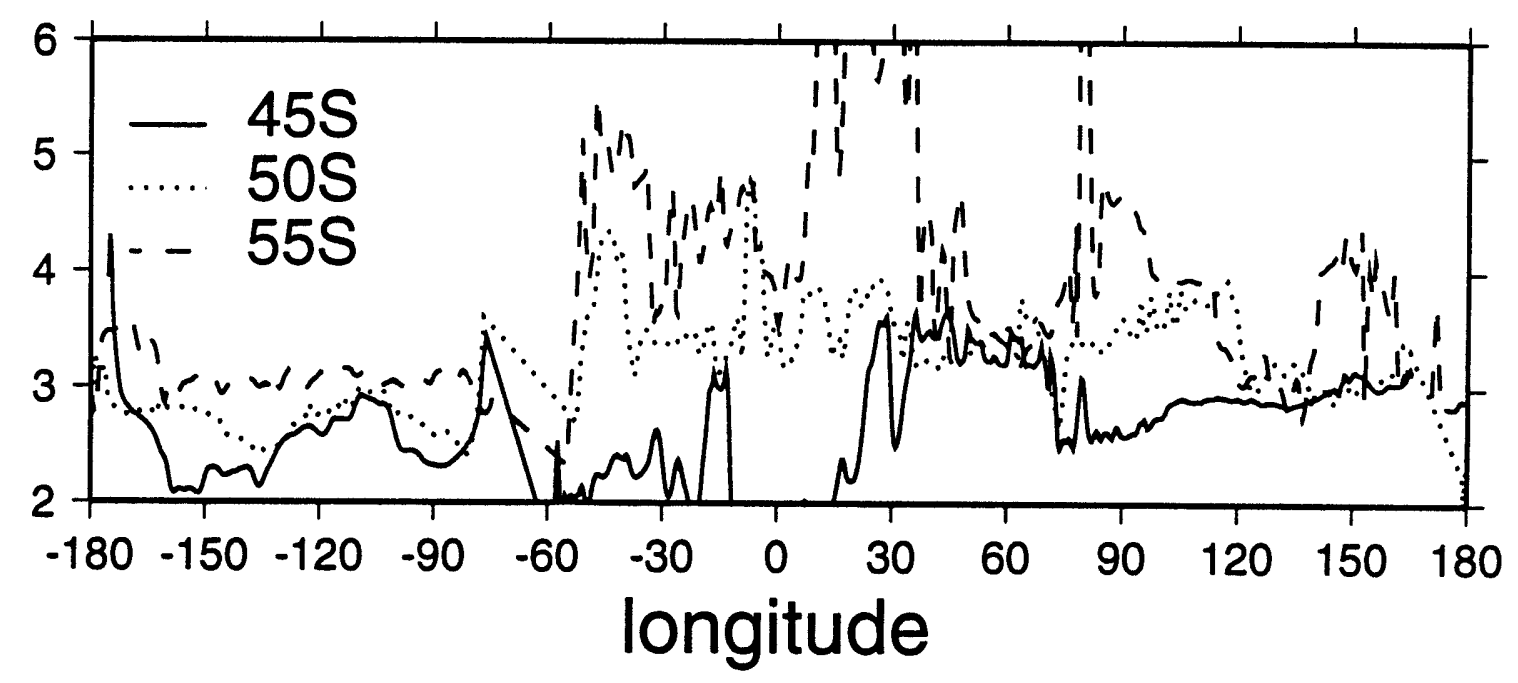

Figure 5.6: Potential vorticity (divided by $\rho_{o}$, multiplied by $10^{11}$ ) along latitude lines, computed from Semtner-Chervin model output. Latitude lines shown correspond to $45^{\circ} \mathrm{S}, 50^{\circ} \mathrm{S}$, and $55^{\circ} \mathrm{S}$.

In contrast with the gradual changes in potential vorticity along streamlines in Figure 5.4a, along lines of latitude, potential vorticity undergoes dramatic fluctuations, particularly at Drake Passage $\left(60^{\circ} \mathrm{W}\right)$ and in the central Indian Ocean $\left(30^{\circ} \mathrm{E}\right)$, as shown in Figure 5.6. Thus in comparison with the large zonal variations in potential vorticity, it is nearly conserved along streamlines. The variations which occur are indications of minor inhomogeneities in the forcing of the ACC. These subtle variations can be explained by probing the physical processes governing potential vorticity.

The surface $\sigma_{1}=32.3$ is at mid-depth between 1500 and $500 \mathrm{~m}$ depth throughout most of the ACC, as shown in Figure 5.7. Thus the isopycnal layer is well below the ocean's surface and is not directly wind forced. Instead the wind stress acts by displacing isopycnal surfaces through Ekman pumping. Figure 5.7b indicates that the layer thickness decreases as the layer shallows. Since the isopycnals are tilted, a thin layer suggests a broad density gradient, while a thick layer may correspond to more steeply tilted isopycnals and a narrower jet. Since the isopycnal surface is deeper where the ACC is further south, in the Pacific Ocean, this suggests that the 

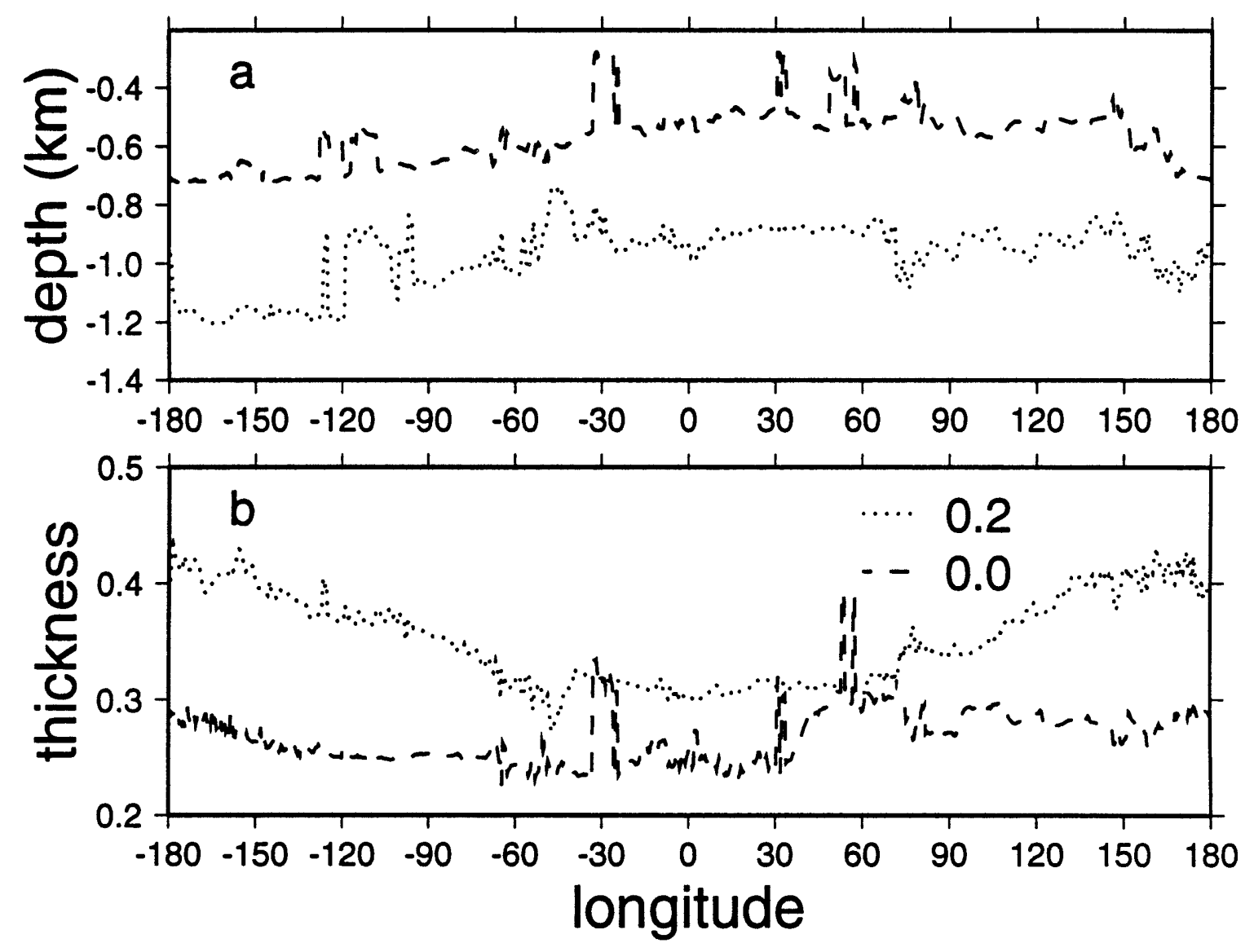

Figure 5.7: (a) Depth of the $\sigma_{1}=32.3$ isopycnal surface in $\mathrm{km}$ along streamlines. (b) Thickness of the isopycnal layer corresponding to $32.25<\sigma_{1}<32.35$, in $\mathrm{km}$. 
flow deepens as it thickens and narrows, in accord with the decreasing Rossby radius at higher latitudes.

To understand what controls these variations in potential vorticity, compare the value of potential vorticity with the forcing terms controlling it, defined by (5.10). Integrating over noisy quantities introduces substantial errors; the relative size of the terms nonetheless serves as an indicator of oceanic forcing and response. The terms shown in Figure 5.8 indicate that the only component of the potential vorticity balance large enough to significantly affect potential vorticity along streamlines is the crossisopycnal Ekman pumping term. The horizontal viscosity term and ageostrophic term are nearly constant across most of the domain, but undergo sharp changes at Drake Passage, which may represent numerical problems. Similar numerical problems also affect the Ekman pumping term. Two terms are omitted from Figure 5.8: transient eddy terms cannot be computed from the available data, and the vertical viscosity term is too small to have any significant impact on the vorticity balance. Nonetheless, the results suggest that the observed changes in potential vorticity along streamlines may be explained by cross-isopycnal Ekman pumping and the unavailable transient eddy fluxes.

\subsubsection{Isopycnal Potential Vorticity Estimates from Data}

The same calculation performed using the Olbers et al. [1992] atlas data produces slightly different results. Figure 5.9 shows the Montgomery potential on the isopycnal surface $\sigma_{1}=32.3$. Potential vorticity on the $\sigma_{1}=32.3$ surface from atlas data is shown in Figure 5.10. Mapping potential vorticity onto Montgomery streamlines, as shown in Figure 5.11, suggests that potential vorticity varies differently along streamlines than the numerical model output would suggest, undergoing less dramatic large scale shifts in density structure along its length, but more substantial variations not only at Drake Passage and Campbell Plateau, but also at a number of other ridges throughout the system. Non-synopticity and substantial smoothing make the atlas data a sub- 


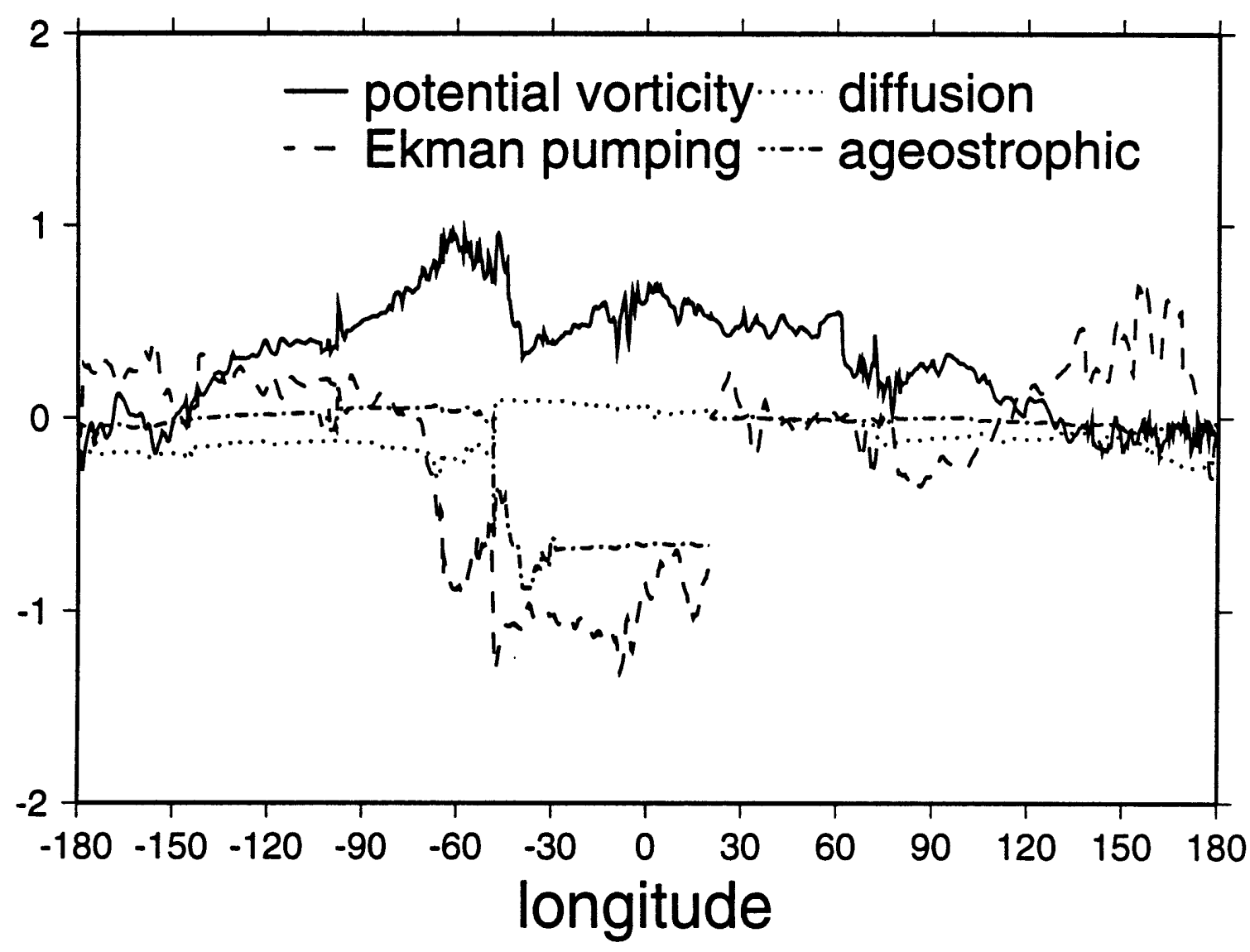

Figure 5.8: Potential vorticity along the $0.2 \mathrm{~m}$ Montgomery streamfunction (solid line) compared with forcing terms due to cross-isopycnal Ekman pumping (dashed line), horizontal viscosity (dotted line), and ageostrophic effects (dash-dotted line). Note that model integration begins at $20^{\circ} \mathrm{E}$. The vertical viscosity term is too small to have any significant impact, while transient eddy terms cannot be computed from the available data. 


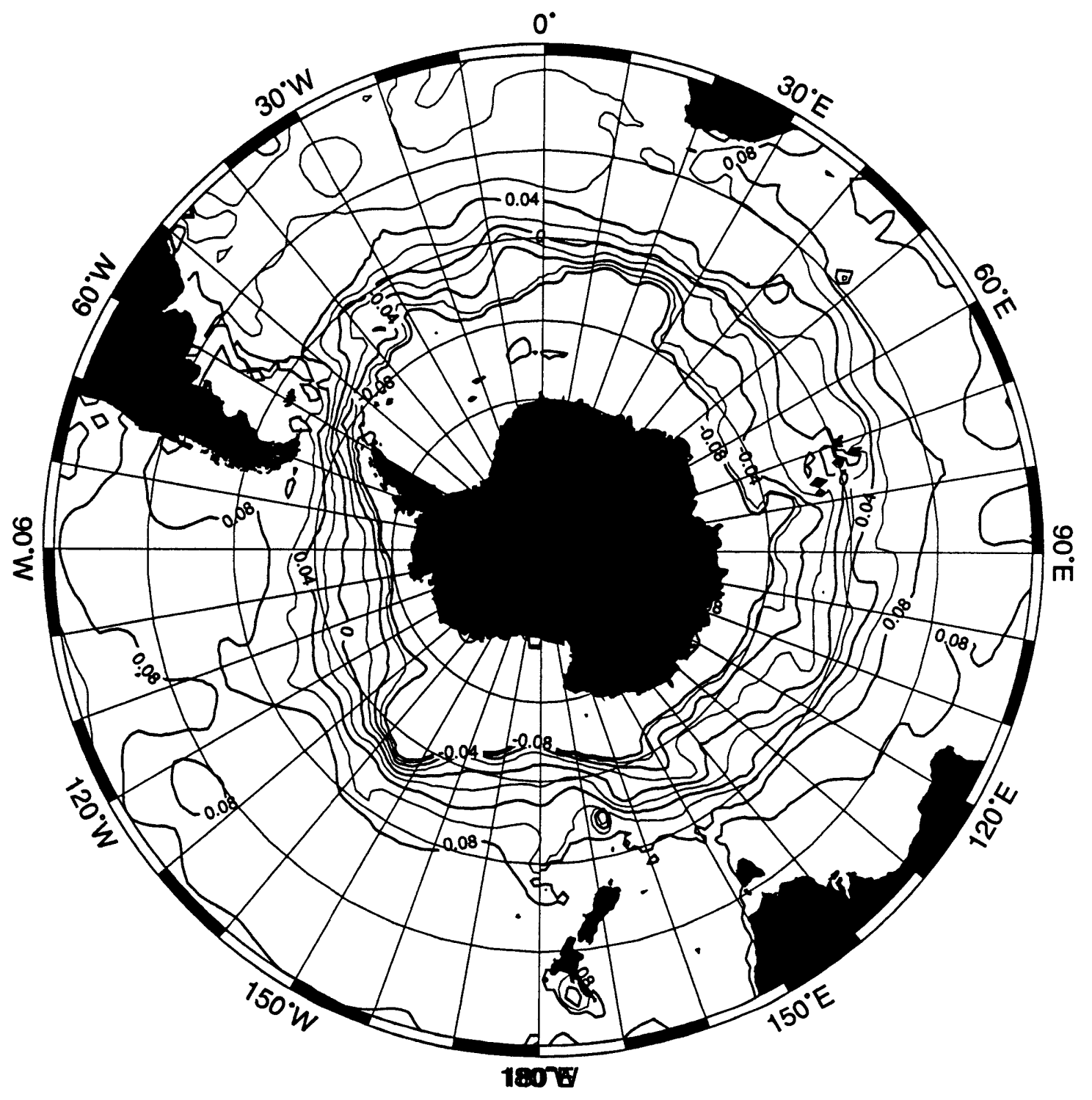

Figure 5.9: Montgomery potential, $M$, as defined in (5.4) in $\mathrm{m}$, on the isopycnal surface $\sigma_{1}=32.3$ referenced to the isopycnal surface $\sigma_{1}=32.4$. The contour interval is $0.02 \mathrm{~m}$, and the total range, from $-0.08 \mathrm{~m}$ to $+0.08 \mathrm{~m}$, is small because of the relatively shallow reference layer, but should nonetheless be representative of physical processes. 


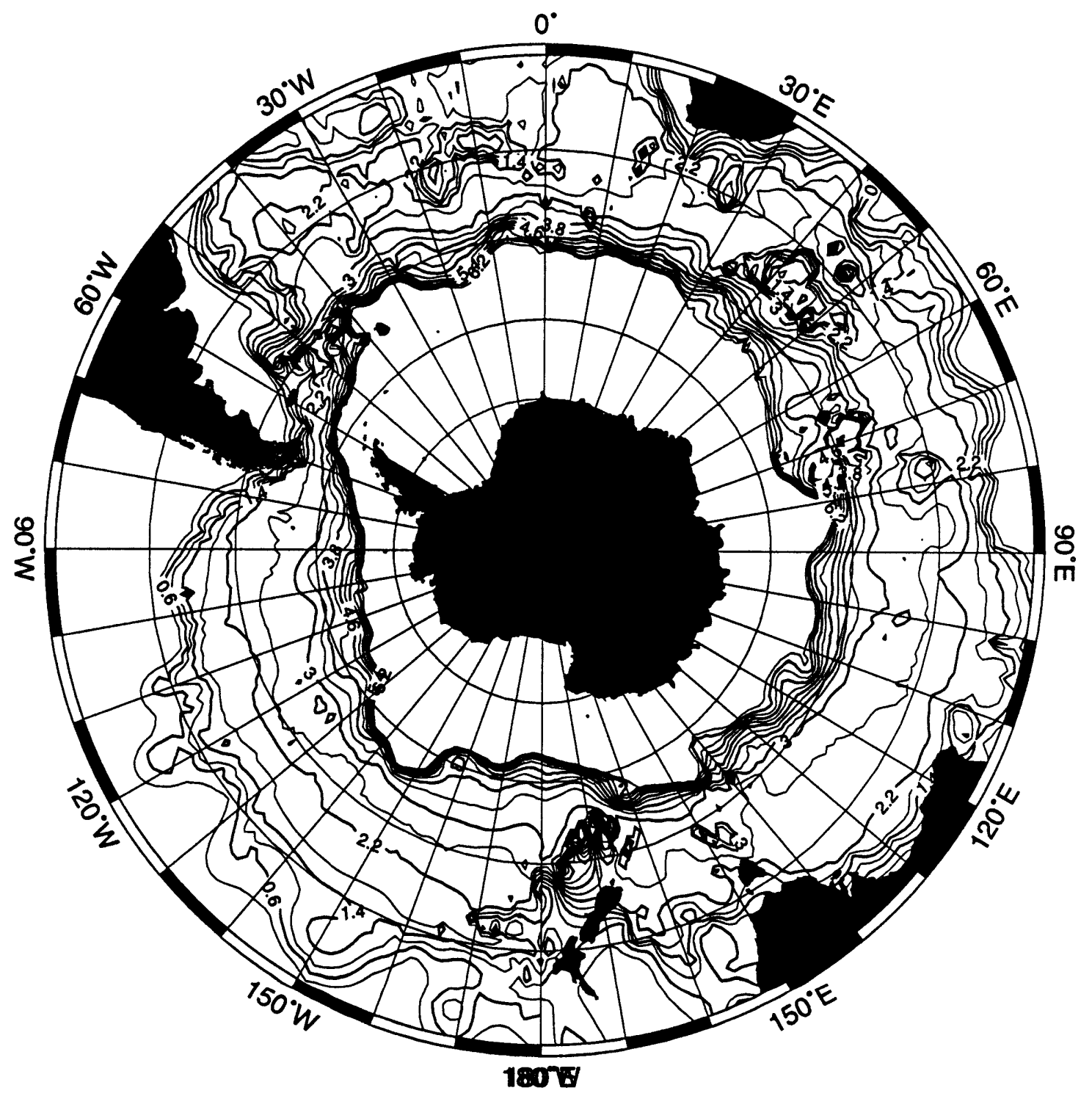

Figure 5.10: Potential vorticity, $f \frac{\partial \sigma}{\partial z}$ (in $\mathrm{kg} \mathrm{s}^{-1} \mathrm{~m}^{-4} \times 10^{8}$ ), on the $\sigma_{1}$ surface 32.3 determined from the gridded Olbers et al. [1992] atlas data. Data is smoothed by fitting an exponential decay function to the values of $\sigma_{1}$ computed from the atlas data. 


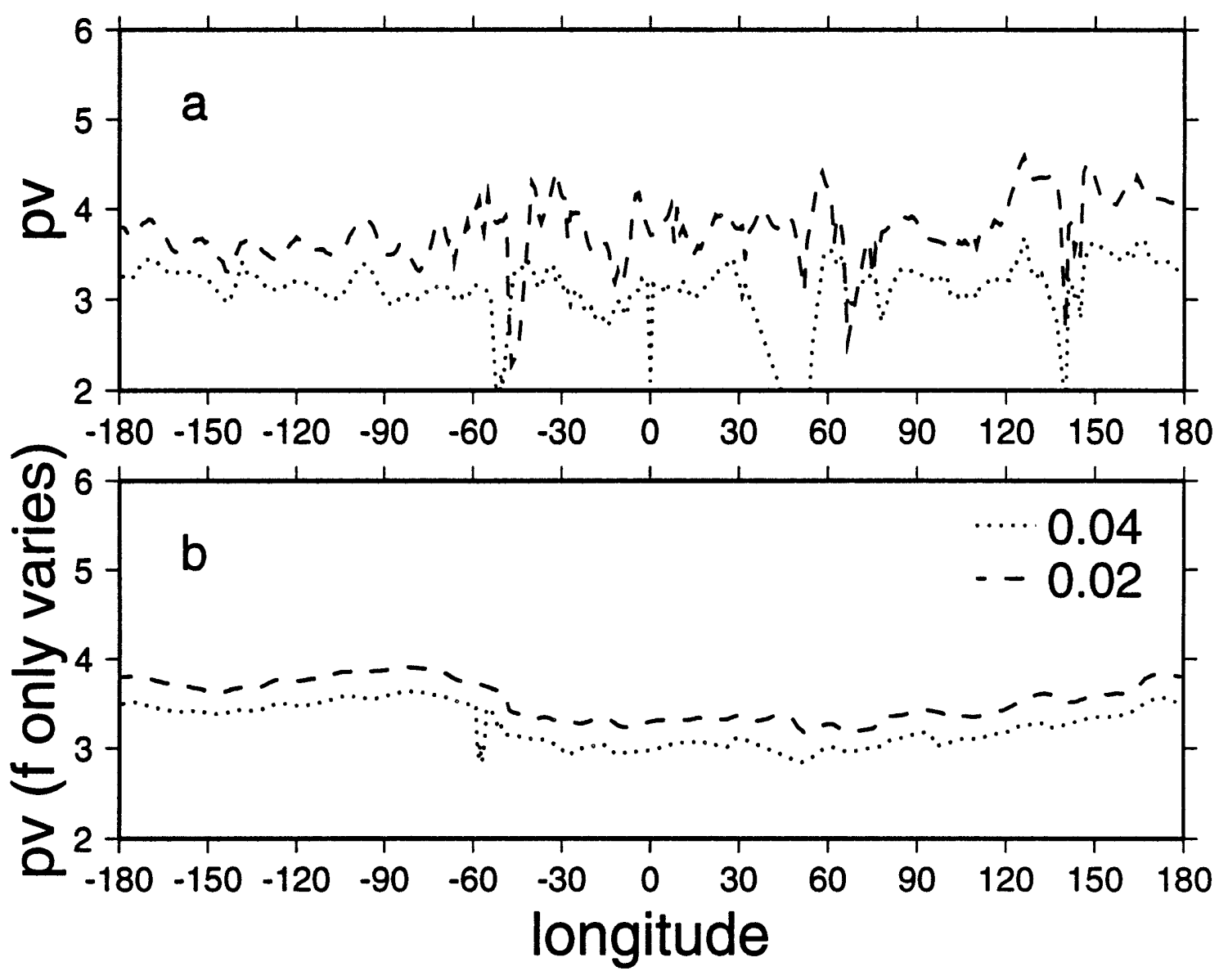

Figure 5.11: (a) Potential vorticity (in $\mathrm{kg} \mathrm{s}^{-1} \mathrm{~m}^{-4} \times 10^{8}$ ) along Montgomery streamlines determined from the Olbers et al. [1992] atlas data. Height contours correspond to the contours labeled 0.04 and 0.02 in Figure 5.9. (b) Potential vorticity along Montgomery streamlines when $\frac{\partial z}{\partial \sigma}$ is held fixed so that only the effects of changing latitude are assessed. 
optimal proxy for potential vorticity and streamfunctions. The differences between Figures 5.4 and 5.11 may simply be a result of inadequate sampling in atlas data; however, if the atlas data are representative of Southern Ocean stratification then the results indicate that the ACC undergoes substantial changes in potential vorticity over relatively short distances, perhaps associated with the locations of intermediatescale topographic features. Thus wind stress curl may be dissipated at many more topographic features in the ocean than it is in the numerical model.

Isopycnal coordinates have a clear physical interpretation, since mixing is predominantly along potential density surfaces; however, comparisons with satellite measurements are easier if we look at potential vorticity using a formulation appropriate for surface streamlines. The next section will investigate how potential vorticity may be examined on surface height contours.

\subsection{Potential Vorticity on Surface Streamlines}

\subsubsection{An Equivalent Barotropic Form}

Although isopycnal coordinates succinctly illustrate potential vorticity structures, satellite measurements show only surface phenomena and are better interpreted on surface streamlines. Marshall [1994a,b] has developed one strategy to relate potential vorticity to surface streamlines. This discussion will present a scheme which differs from Marshall's work primarily because a level of no motion is not imposed. Both formulations will be applied to the available Southern Ocean data, and the assumptions implicit in these formulations will be assessed.

From the basic equations of motion, the vorticity equation on a level is:

$$
\zeta_{t}+\mathbf{u} \cdot \nabla(\zeta+f)-f w_{z}=\mu \frac{\partial^{2} \zeta}{\partial z^{2}}+A_{H} \nabla^{4} \zeta
$$


where $\zeta$ is the relative vorticity, and the wind stress curl enters through the upper level boundary condition:

$$
\mu \frac{\partial \zeta}{\partial z}=\frac{\operatorname{curl} \tau}{\rho_{\mathrm{o}}} \text { at } z=0 .
$$

This equation compactly describes the upper ocean vorticity balance, but it does not represent potential vorticity. To obtain the conservation property associated with potential vorticity, the equation must be transformed to eliminate the Ekman pumping term $f \frac{\partial w}{\partial z}$.

Time averaging and vertically integrating (5.12), and eliminating the nonlinear terms, produces an expression for the vorticity:

$$
\beta V-f \mathbf{u}_{b} \cdot \nabla H=\frac{\operatorname{curl} \tau}{\rho_{\mathrm{o}}}+A_{H} \int_{H}^{\eta} \nabla^{2} \zeta_{\mathrm{s}} .
$$

where $V$ is the vertically integrated meridional transport, $\mathbf{u}_{b}$ is the bottom velocity, $\zeta_{0}$ represents relative vorticity, and $H$ is the bottom depth. ${ }^{2}$ Thus the wind stress curl and other forcing terms are balanced by changes in planetary vorticity, represented by $\beta V$, and by an induced vertical velocity at the bottom, essentially an Ekman pumping caused by bathymetry.

While the vorticity equation itself does not have a simple closed form solution, observational evidence suggests plausible simplifications to compress it into a form easily interpreted at the ocean surface. First, analysis of current meter and transport mooring data in Drake Passage indicates that the ACC is predominantly geostrophic [Whitworth and Peterson, 1985]. This confirms that the Rossby number, $R_{o}=\frac{U}{f L}$, is small and will justify use of quasi-geostrophic scaling arguments.

Second an extensive body of research indicates that the ACC has a self-similar, or equivalent barotropic, vertical structure. Current meters deployed in the Drake Passage have indicated that fluctuations of the Southern Ocean are vertically coher-

\footnotetext{
${ }^{2}$ The joint effect of baroclinicity and relief (JEBAR) term, which is a significant factor in some vertically integrated vorticity equations, does not appear here because the vorticity equation is formed by taking the curl of the momentum equation and then depth averaging, instead of the inverse. Mertz and Wright [1992] provide a clear discussion of the distinction.
} 
ent, although the current structure itself has a strong vertical shear: Whitworth and Peterson [1985] compared fluctuations in the meridionally integrated velocity estimated from pressure gauge measurements at $500 \mathrm{~m}$ depth with the total transport through Drake Passage estimated from transport moorings in combination with pressure gauge measurements and historic hydrographic measurements; they found that the two time series were coherent at the $95 \%$ confidence level. Pillsbury et al. [1979] compared current meter velocities in Drake Passage at three depths, and found that both horizontal components of velocity were correlated in the vertical with greater than $99 \%$ confidence. Current meters placed in the gap between the Georgia and Argentine basin also showed a strong vertical coherence [Whitworth et al., 1991]. In addition, hydrographic data is consistent with the current meter measurements. Lutjeharms's [1977] detailed investigation of historic data for the Southern Ocean indicated that density fluctuations tend to be strongly coherent throughout the water column. Gordon et al. [1978] found that normalized geostrophic velocity profiles at $95^{\circ} \mathrm{W}$ and $85^{\circ} \mathrm{E}$ were nearly the same for all station pairs and showed a distinctive exponential vertical structure. Numerical model results also indicate that surface and deep flow are closely related; Killworth [1992] observed that the FRAM data showed an equivalent barotropic structure with correlations between upper layer velocities and lower layer velocities consistently greater than 0.5 and with a roughly exponential vertical velocity structure.

This combination of observational and numerical evidence suggests that knowing the velocity at the surface is sufficient to estimate the flow field everywhere. For the purposes of this study, a general equivalent barotropic velocity structure is assumed of the form:

$$
\mathbf{u}(x, y, z, t)=\mathbf{u}_{\bullet}(x, y, t) \phi(z)+\mathbf{u}_{c}(x, y, z, t),
$$

where $\mathbf{u}$ is the absolute velocity, $\mathbf{u}_{\mathbf{s}}$ is the geostrophic surface velocity, $\phi$ represents the vertical attenuation of velocity, and at the surface $\phi(0)=1$. The term $\mathbf{u}_{c}$ is a small correction, assumed to be on the order of the Rossby number, $R_{0}$. 
The vertically integrated vorticity equation can be converted into a simple expression representing conservation of potential vorticity by assuming a known vertical structure, such that the velocity at any depth depends only on the surface velocity and the depth. Gordon et al.'s [1978] and Killworth's [1992] analyses suggest a self-similar vertical structure with exponential decay properties. Hence, define:

$$
\phi(z)=\exp \left(\frac{z}{H_{0}}\right) .
$$

$H_{0}$ the e-folding depth, is equivalent to the upper layer depth if the system is treated as a $1 \frac{1}{2}$ layer system with an infinitely deep lower layer. Such a structure, implicitly imposes an infinitely deep level of no motion, $H_{\text {ref }}$. Although there is no a priori reason to fix $H_{\text {ref }}$ to an infinite rather than finite depth, observational work has frequently used the bottom as a reference level (eg. Georgi and Toole [1982]) suggesting that deep reference levels are appropriate. (As the appendix illustrates, this vertical structure is nearly identical to the assertion that potential vorticity is a linear function of density, so that $Q=f \frac{\partial \rho}{\partial z}=a\left(\rho-\rho_{\infty}\right)$ as suggested by Marshall et al. [1993] and employed by Marshall [1994a,1994b].) For convenience, the vertical integral of $\phi(z)$ is defined as $\Phi$. Thus:

$$
\Phi=\int_{-H}^{0} \phi(z) d z=H_{\circ}\left[1-\exp \left(-\frac{H}{H_{o}}\right)\right],
$$

for an ocean where vertical velocities due to variations in the sea surface $\eta$ are significantly smaller than those due to variations in the bottom topography. The function $\Phi(H)$ may be thought of as representing the equivalent depth of the upper layer in a $1 \frac{1}{2}$ layer model, adjusted for ocean depth.

Employing the self-similar exponential velocity structure implied by (5.15) and (5.16), and rewriting the vorticity equation, (5.14) produces

$$
\frac{g}{f} J\left(\eta, \frac{f}{\Phi}\right) \Phi^{2}=\frac{\operatorname{curl} \tau}{\rho_{o}}+A_{H} \int_{H}^{\eta} \nabla^{2} \zeta .
$$

Thus in the absence of forcing, the quantity $f / \Phi$ is conserved along surface streamlines and may be viewed as a proxy for potential vorticity in this self-similar formulation. 
Inherent in this result is an assumption that $H_{0}$ varies much more slowly than the bottom depth $H$ and that aside from a small and unimportant bottom boundary layer, bottom velocities are determined solely from the exponential decay of surface velocity with depth. From this proxy for potential vorticity, $f / \Phi$, the more familiar barotropic case may be derived by allowing $H_{0}$ to be infinite; in this case $\Phi$ reduces to $H$, and potential vorticity is represented by $f / H .^{3}$

Marshall [1994a] derived a result analogous to (5.18), where with no viscosity or wind stress curl:

$$
\mathbf{k} \wedge \nabla\left(\left\{H-H_{r e f}\right\} e^{-\frac{a H}{f}}+\frac{f}{a}\left\{e^{-\frac{a H}{f}}-1\right\}\right) \cdot \nabla \rho_{s}=0 .
$$

Hence, along surface streamlines defined by contours of $\rho_{s}$, the quantity

$$
\tilde{Q}=\left\{H-H_{\text {ref }}\right\} e^{-\frac{a H}{f}}+\frac{f}{a}\left\{e^{-\frac{a H}{f}}-1\right\}
$$

is conserved. $\tilde{Q}$ may be interpreted as a proxy for potential vorticity, although it has units of depth. Three distinctions between (5.21) and (5.18) are discussed in greater detail in the appendix. First, surface density contours, $\rho_{s}$, are substituted for surface height contours, $\eta$. Second, while (5.18) assumes an infinitely deep level of no motion, Marshall's work requires a level of no motion at depth $H_{\text {ref. }}$. The term $\left(H-H_{\text {ref }}\right) \exp \left(-\frac{a H}{f}\right)$ is therefore a barotropic correction for this level of no motion. Finally, the term $\frac{f}{a}\left(\exp \left(-\frac{a H}{f}\right)-1\right)$ is equivalent to $\Phi$ in (5.18), where $H_{0}$ is $\frac{f}{a}$. The different dependence on Coriolis parameter in (5.21) results from the slightly

\footnotetext{
${ }^{3}$ The reintroduction of relative vorticity complicates this formulation. If relative vorticity were barotropic, potential vorticity could be expressed as $Q=\frac{t+\zeta}{\Phi}$, but if we assume, more plausibly, that relative vorticity attenuates like velocity with depth, then the nonlinear effects alter the expression, and the appropriate equation is:

$$
\frac{g}{f} J\left(\eta, \frac{\zeta}{\Theta}\right) \Theta^{2}+\frac{g}{f} J\left(\eta, \frac{f}{\Phi}\right) \Phi^{2}=\frac{\operatorname{curl} \tau}{\rho_{o}}+A_{H} \int_{H}^{\eta} \nabla^{2} \zeta
$$

where

$$
\Theta(H)=\int_{-H}^{0} \phi(z)^{2} d z
$$

which represents the equivalent depth for the nonlinear terms.
} 
different velocity structure introduced by fixing the vertical structure of potential vorticity rather than velocity. Both (5.18) and (5.21) will be employed to examine the altimeter and model output on surface streamlines.

\subsubsection{Model and Data Surface Potential Vorticity}

Before examining approximations for potential vorticity on surface streamlines, first consider the upper ocean vorticity balance in the Semtner-Chervin model output. Using the same procedure as in section 5.2.2, the vorticity equation (5.12) is integrated to compare the vorticity $(f+\zeta)$ with its forcing terms at $87.5 \mathrm{~m}$ depth in Figure 5.12. Dramatic variations in the vorticity are not explained by viscosity, ageostrophic effects, or either of two smaller effects which are not shown: the long term tendency of $\zeta$ and vertical viscosity. The magnitude of the transient eddy term cannot readily be calculated from the model statistics because the necessary correlation terms are not stored in the model output. Substantial errors in integrating along streamlines prevent accurate computation of the vertical Ekman pumping term, $f \frac{\partial w}{\partial z}$, but the overall magnitude of the term suggests that it must be responsible for the large scale trends in vorticity. Thus, surface wind stress curl generates Ekman pumping which decreases the vorticity, $f+\zeta$, driving the flow southwards across the Indian and Pacific Oceans. Vorticity is relatively constant in the Atlantic, suggesting that the streamline examined in Figure 5.12 closely follows the ECMWF zero wind stress curl line. The dramatic shift in the vorticity downstream of Drake Passage $\left(50^{\circ} \mathrm{W}\right)$ suggests that vorticity put in throughout the Southern Ocean is dissipated rapidly in a western boundary current process, possibly due to the transient eddy term. While below the surface vorticity in the upper ocean is not directly wind forced, its changes along streamlines are primarily due to Ekman pumping.

In contrast, potential vorticity conceptually represents both Ekman pumping and vorticity. By examining proxies for potential vorticity along surface streamlines, represented by (5.18) and (5.21), we can see how the full water column responds to the 


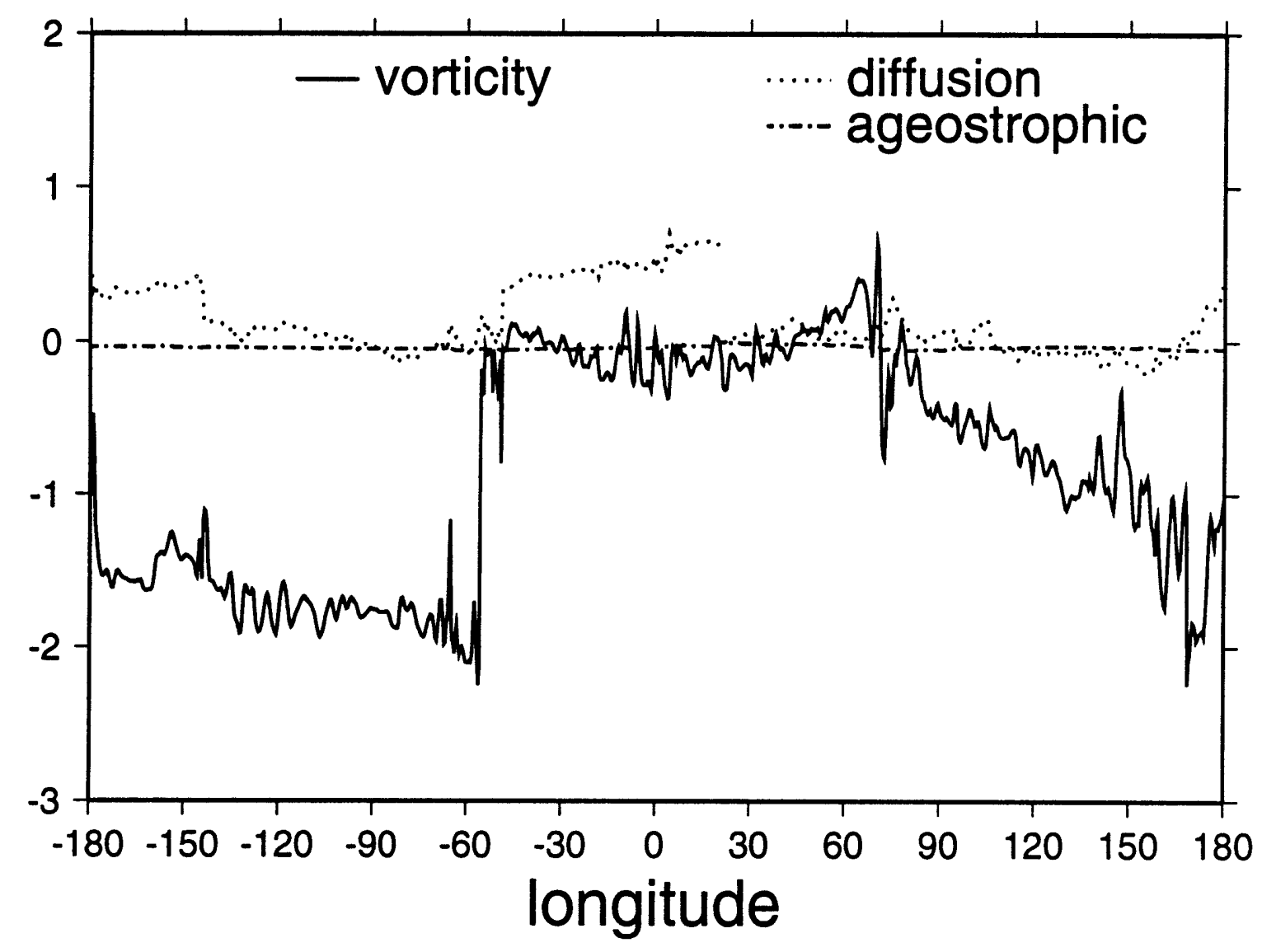

Figure 5.12: Vorticity along a pressure contour running through the core of the ACC at level $4(87.5 \mathrm{~m})$ in the Semtner-Chervin model. Vorticity (solid line) is defined as $f+\zeta$. Also shown are forcing terms corresponding to (dotted line) viscosity $\frac{1}{u_{0}} \int A_{H} \nabla^{4} \zeta d s$, and (dash-dotted line) ageostrophic effects $\frac{1}{u_{0}} \int u_{a g} \cdot \nabla(f+\zeta) d s$. 
vorticity input directly by the wind. In addition employing the proxies for potential vorticity allows comparison of potential vorticity variations along surface streamlines in altimeter and model data. Figure 5.13 shows the quantity $f / \Phi$ defined in (5.18) as a function of longitude along four surface height contours. The e-folding scale, $H_{o}$, of $1.5 \mathrm{~km}$, is an average of values estimated by nonlinearly fitting an exponential decay scale the model velocities and atlas geostrophic velocities. Both the model and data indicate similar variations in potential vorticity along streamlines, which is not surprising since the model and altimeter surface height contours encounter comparable topography and undergo similar latitudinal displacements. Some of the variation is due to changes in the Coriolis parameter, $f$, as the axis of the ACC shifts meridionally along its circumpolar path, but as Figure 5.14 suggests, large fluctuations in the value of $f / \Phi$ occur in response to major topographic features, including in particular, Drake Passage $\left(60^{\circ} \mathrm{W}\right)$, the Crozet Plateau system $\left(50^{\circ} \mathrm{E}\right)$, and Campbell Plateau $\left(170^{\circ} \mathrm{E}\right)$. The difference between potential vorticity upstream and downstream of the topographic features indicates the impact that these features have on the dynamics.

In contrast to the results shown in Figure 5.13, the formulation (5.21) defined by Marshall [1994b] uses an e-folding depth which is on average about $1.7 \mathrm{~km}$, slightly deeper than $1.5 \mathrm{~km}$ used in the Figure 5.13, and which varies as a function of $f$. In addition it employs a different parameterization for the bottom velocity. The resulting proxy for potential vorticity $\tilde{Q}$, in Figure 5.15, shows large spikes at locations where major topographic features intersect the flow, but otherwise varies much like $f$. The difference between potential vorticity upstream and downstream of the topographic features indicates the impact that these features have on the dynamics. Qualtitatively, the results shown in Figures 5.13 and 5.15 are quite similar as both potential vorticity proxies undergo substantial changes in value associated with major changes in latitude in the flow. The results shown in Figure 5.13 are more sensitive to variations in bottom 

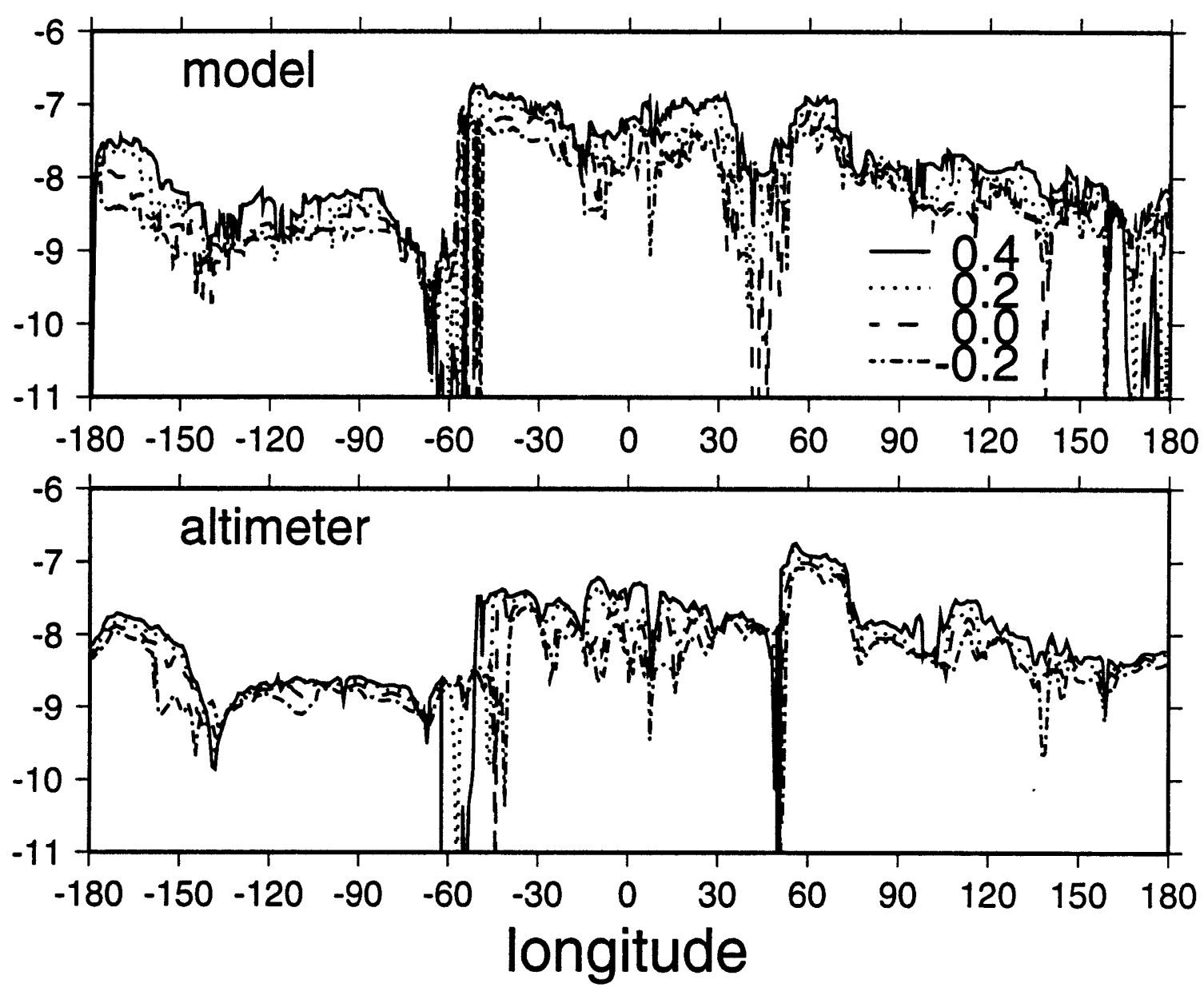

Figure 5.13: The proxy for potential vorticity, $f / \Phi$, defined by (5.18), along sea surface height contours, $\eta$, from the Semtner-Chervin model (top) and altimeter data (bottom). The quantity $f / \Phi$ is in units of $\mathrm{m}^{-1} \mathrm{~s}^{-1}$, multiplied by $10^{8}$. Height contours shown correspond to $0.4,0.2,0$, and $-0.2 \mathrm{~m}$, where the $0 \mathrm{~m}$ contour is fixed to correspond approximately to the center of the ACC. The equivalent depth, $H_{0}$ is fixed at $1.5 \mathrm{~km}$, based on an analysis of model output and atlas data. 


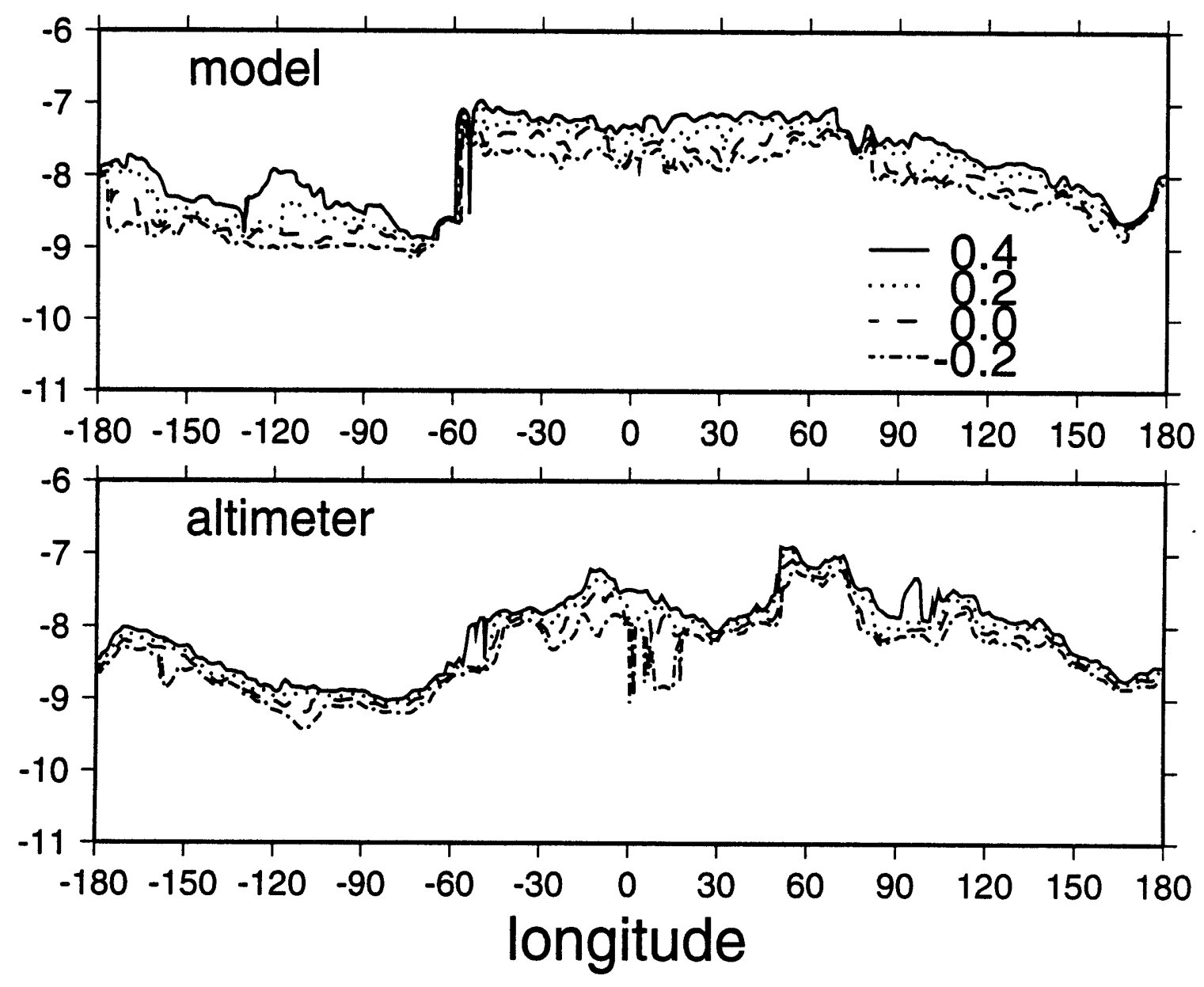

Figure 5.14: Changes in the proxy for potential vorticity, $f / \Phi$, due only to the Coriolis parameter $f$, along sea surface height contours, $\eta$, from the Semtner-Chervin model (top) and altimeter data (bottom). The plot is analogous to Figure 5.13, but $H$ is fixed at $4 \mathrm{~km}$, so that $\Phi$ is constant. 

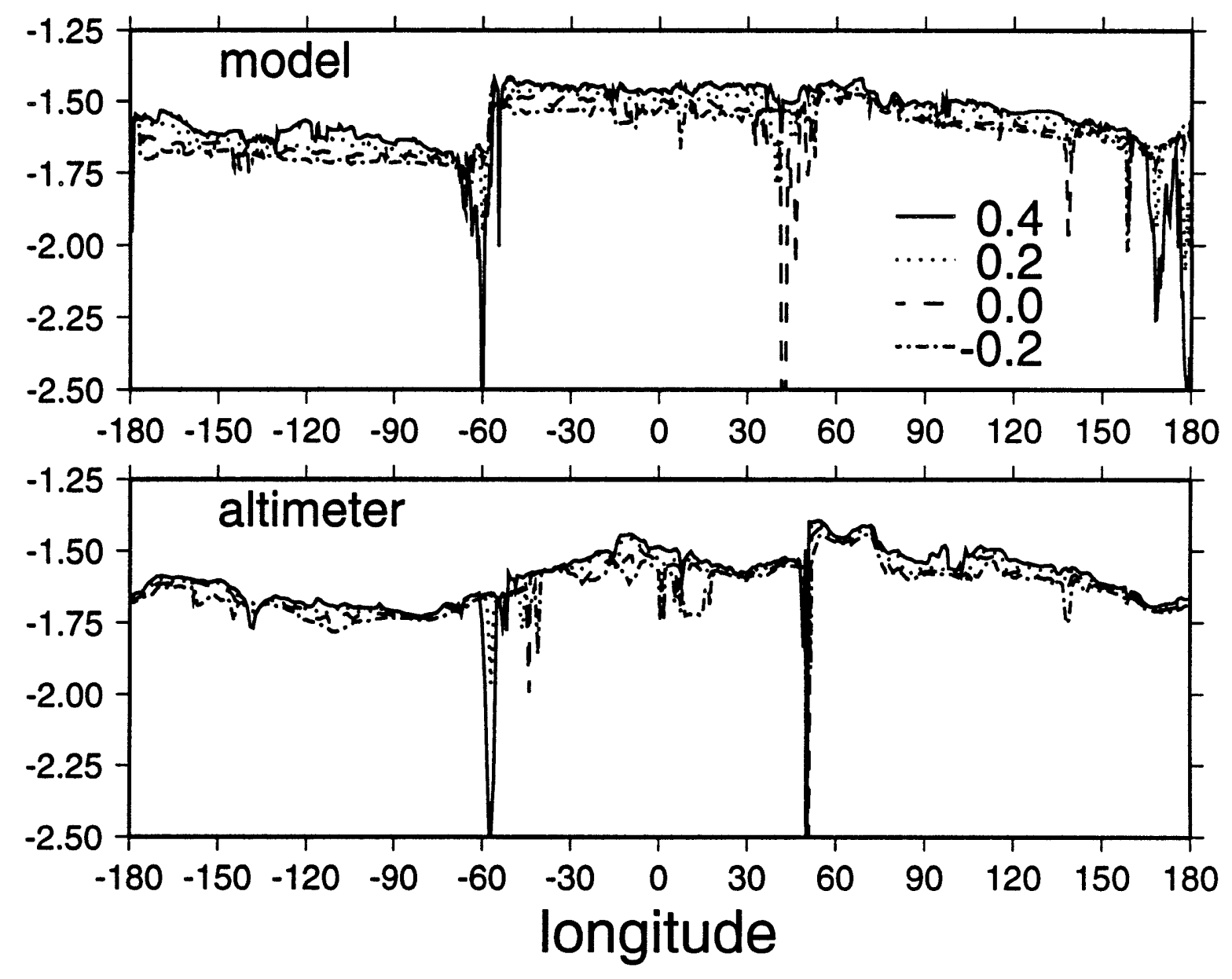

Figure 5.15: The proxy for potential vorticity, $\tilde{Q}$, defined by (5.21) [Marshall, 1994b] along sea surface height contours, $\eta$, from the Semtner-Chervin model (top) and altimeter data (bottom). $H_{\text {ref }}$ is fixed at $4 \mathrm{~km}, a$ is $6.44 \times 10^{-5} \mathrm{~km}^{-1} \mathrm{~s}^{-1} . \tilde{Q}$ is in km. 
topography, while the effect of topography is attentuated in Marshall's formulation in Figure 5.15.

Although these models for potential vorticity along surface streamlines indicate differing sensitivities to bottom topography, neither suggests that potential vorticity is conserved along surface streamlines. Instead, they indicate that surface wind forcing inputs negative vorticity particularly in the Indian and Pacific Oceans, driving the ACC axis southward along most of its path. The wind forcing is dissipated in localized areas associated with major topography. In the Semtner-Chervin model, this occurs almost entirely just downstream of Drake Passage $\left(50^{\circ} \mathrm{W}\right)$, where the flow forms a western boundary current, with smaller effects at Campbell Plateau $\left(170^{\circ} \mathrm{E}\right)$, and the changes in potential vorticity on surface streamlines closely parallel the upper ocean changes in absolute vorticity, shown in Figure 5.12. In the altimeter data, fluctuations in the proxies for potential vorticity near the Crozet Plateau $\left(50^{\circ} \mathrm{E}\right)$ and the EltaninUdintsev Fracture Zone $\left(140^{\circ} \mathrm{W}\right)$ suggest that these features may also help to control the potential vorticity balance.

While these results are suggestive of a Sverdrup-type circulation in which wind stress curl drives a southward flowing ACC which returns in boundary currents at a few isolated locations, the results are quite sensitive to the choice of e-folding depth, $H_{0}$. The e-folding depth used to generate the results Figure 5.13 is a representative depth based on a nonlinear fit to geostrophic velocities from atlas data and model velocity profiles. However, data suggest that $H_{\circ}$ varies by about $30 \%$ along streamlines, as shown in Figure 5.16. Estimated values of $H_{\circ}$ are not well correlated either with the Coriolis parameter, $f$, as Marshall [1994b] assumes, or with ocean depth. The observed significant changes in $H_{0}$ are consistent with layer thickness changes in section 5.2 which substantially accounted for potential vorticity variations along streamlines. Thus the substantial variability in e-folding depth suggests that using a simple constant parameterization for $H_{0}$ may not fully capture oceanic variability. Furthermore, if stratification changes are associated with gradients in topography, as 

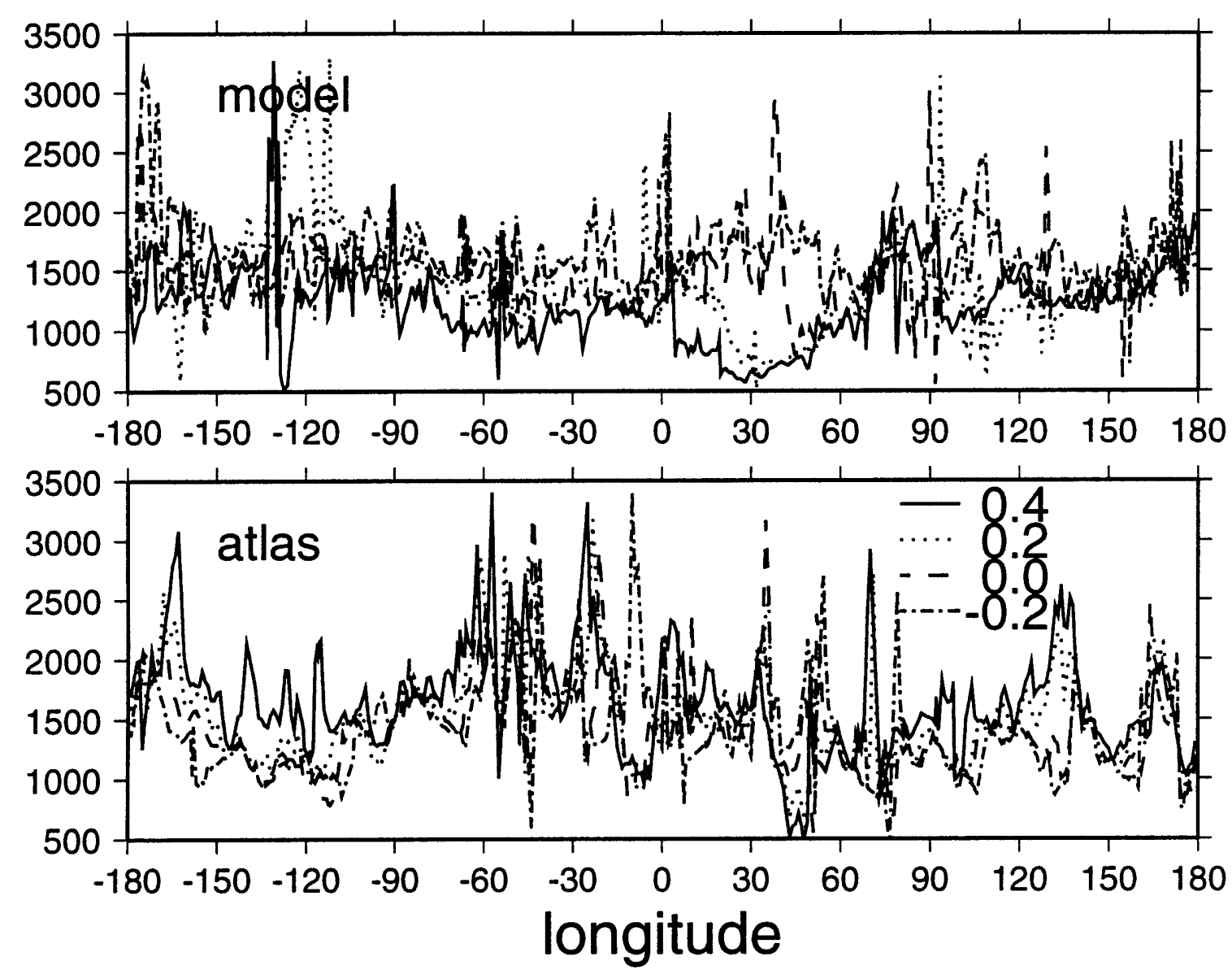

Figure 5.16: The e-folding depth, $H_{o}$, (in $\mathrm{m}$ ) for the model (top) and hydrographic atlas data (bottom). Values are computed by nonlinearly fitting an exponential function to velocity data from the model and geostrophic velocity information from the atlas. Outliers are eliminated in these plots. 
conceptual understandings of form drag might suggest, then the locations of rapid changes in $H_{0}$ should be dynamically significant and should mark the topographic features generating form drag. Such variations are not represented by the simple approximations used in (5.18) and (5.21). In addition, changes in $H_{0}$ on surface streamlines are more dramatic in atlas data than in model data, suggesting variations in stratification occur over shorter distance scales in the ocean than in the model, and thus that the assumptions about $H_{0}$ being constant may be more appropriate for model output than for the real ocean. While surface proxies may capture potential vorticity variations associated with changes in planetary vorticity, they cannot represent subtleties in stratification changes. To fully assess these factors will require more detailed study of relatively synoptic hydrographic measurements.

\subsection{Summary}

Although potential vorticity is often conceived as a conserved quantity, in a forced system it may vary substantially in response to wind and dissipative processes. The results of this analysis suggest that in the Semtner-Chervin model, potential vorticity is not precisely conserved along streamlines on an isopycnal surface at mid-depth. Its changes are largely due to stratification changes rather than changes in the Coriolis parameter, $f$, or variations in relative vorticity. Variations in potential vorticity are attributed to Ekman pumping and transient eddy processes, which together increase potential vorticity in the Atlantic and decrease it across the Indian and Pacific Oceans.

Potential vorticity on surface streamlines is more difficult to define. By assuming a self-similar vertical structure for potential vorticity [Marshall, 1994b] or velocity, a proxy for potential vorticity on surface streamlines is defined. The sensitivity of this conserved quantity to bottom topography depends on the e-folding depth of the flow. The proxies for potential vorticity vary substantially along streamlines, suggesting that surface wind forcing drives the ACC southward over most of its path, and 
that transient eddy processes and other nonlinear boundary current effects return the ACC jets northward at a few isolated locations associated with major topographic obstructions of the flow, most notably at Drake Passage. Neither proxy is able to capture the stratification changes which the full analysis of model potential vorticity suggests are important. Thus, while such measures are suggestive of dynamical processes in the ocean, without further information, they cannot account for the entire potential vorticity budget.

\subsection{Appendix: Alternative Potential Vorticity For- mulation}

The simple assumptions about equivalent barotropic vertical structures in (5.18) are related to Marshall [1994b]'s Southern Ocean circulation scheme, represented by (5.21), based on a linear relation between potential vorticity and density. The differences stem from several minor factors.

To understand this, first consider the relationship between velocity and density. From the hydrostatic relation,

$$
p(z)=\int_{-H_{\text {ref }}}^{z} \rho g d z+p\left(H_{\text {ref }}\right)
$$

so the geostrophic velocity is therefore:

$$
\mathbf{u}_{g}(z)=\frac{1}{f \rho_{\mathrm{o}}} \mathbf{k} \wedge \nabla p(z)=\frac{g}{f \rho_{\mathrm{o}}} \int_{-H_{r e f}}^{z} \mathbf{k} \wedge \nabla \rho d z
$$

where the pressure is assumed to be constant at $H_{\text {ref }}$, so that there is no geostrophic velocity. Marshall et al. [1993] assumed a linear relation between potential vorticity and potential density, so that:

$$
Q(\rho)=f \frac{\partial \rho}{\partial z}=a\left(\rho-\rho_{\infty}\right)
$$


where the density asymptotically approaches $\rho_{\infty}$ at great depth. Glossing over the distinction between density and potential density, Marshall [1994b] derived the relation:

$$
\rho-\rho_{\infty}=\left(\rho_{s}-\rho_{\infty}\right) e^{\frac{a 2}{f}}
$$

where $\rho_{s}$ is the surface density. Using this expression for density, the geostrophic velocity is:

$$
\mathbf{u}_{g}(z)=-\frac{g}{f \rho_{o}} \mathbf{k} \wedge \nabla\left[\rho_{s} \frac{f}{a}\left(e^{\frac{a z}{f}}-e^{-\frac{a H_{r e f}}{f}}\right)\right] .
$$

This expression differs from (5.15) and (5.16) only in that a level of no motion, $H_{\text {ref }}$, is defined and that the e-folding depth $\frac{f}{a}$ varies with latitude. While (5.18) uses an infinitely deep level of no motion, Marshall sets $H_{\text {ref }}$ to be $4 \mathrm{~km}$, allowing reverse flow below that depth. The variation in e-folding depth with latitude comes from using potential vorticity, which depends on $f$, to fix a vertical structure for the flow, rather than velocity itself. Marshall defines:

$$
H_{1}=\frac{f}{a}=\frac{f}{-6.44 \times 10^{-8} \mathrm{~m}^{-1} \mathrm{~s}^{-1}} .
$$

which translates into an e-folding depth of about $2 \mathrm{~km}$. A close examination of the hydrographic data [Olbers et al., 1992] suggests that this may be a little deep. The different vertical structure used to develop (5.18) includes no dependence on $f$ in the e-folding depth $H_{o}$, but data indicate appropriate values of $H_{o}$ and $H_{1}$ should be comparable. The differences in $H_{\text {ref }}$ and the e-folding depth result in several differences between the results derived in this chapter (5.18) and Marshall's [1994b] result (5.21).

First, in (5.18) surface streamlines are defined by $\eta$, while in (5.21) they are represented by $\rho_{s}$. The difference between the two is small. Consider the surface geostrophic velocity. From the geostrophic velocity (5.27) the surface velocity is

$$
\mathbf{u}_{s}=-\frac{g}{f \rho_{o}} \mathbf{k} \wedge \nabla\left[\rho_{s} \frac{f}{a}\left(1-e^{-\frac{a H_{r e f}}{f}}\right)\right] .
$$


The geostrophic surface velocity can also be represented using the gradient of the sea surface height, $\eta$ :

$$
\mathbf{u}_{\mathbf{s}}=\frac{g}{f} \mathbf{k} \wedge \nabla \eta
$$

If $H_{r e f} \rightarrow-\infty$ and $\frac{f}{a}$ is constant, the only difference between the surface velocities is represented by the differences between contours of $\rho_{s}$ and contours of $\eta$. Combining (5.29) and (5.30) under these conditions produces a surface density conservation principle:

$$
\mathbf{u}_{s} \cdot \nabla \rho_{s}=0
$$

By imposing a simple self-similar vertical structure, we require that contours of $\rho_{s}$ and $\eta$ be parallel, and thus constrain the surface density structure. Bottom velocities must be determined entirely based on the surface condition in such a system. Buoyancy forcing and ageostrophic effects are both sources of error in this approximation. In contrast, Marshall [1994b] imposes a finite value of $H_{\text {ref }}$ and in accordance with (5.25) requires $a$ to be constant so that $\frac{f}{a}$ varies with $f$. In this case the surface conservation principle is:

$$
\mathbf{k} \wedge \nabla \eta \cdot \nabla\left[\frac{\rho_{s}}{\rho_{o}} \frac{f}{a}\left(1-e^{-\frac{a H_{r e f}}{f}}\right)\right]=0 .
$$

or to examine flow along contours of $\rho_{s}$ :

$$
\mathbf{k} \wedge \nabla\left[\frac{\eta-\bar{\eta}+\overline{\frac{\rho_{o} f}{\rho_{o} a}\left(1-e^{-\frac{a H_{r e f}}{f}}\right)}}{\frac{t}{a}\left(1-e^{-\frac{a H_{r e f}}{f}}\right)}\right] \cdot \nabla \rho_{\mathbf{s}} \approx 0
$$

where overbars represent spatial means. By removing the means from the quantities we are investigating, we can better compare them. These results show that an identifiable relationship links $\eta$ and $\rho_{s}$ in Marshall's work, but the gradients of the two quantities are not exactly equivalent. Under most circumstances with a deep level of no motion and minimal variations in latitude along streamlines, the differences between $\rho_{s}$ and $\eta$ should nonetheless be quite small. Since $\eta$ is readily available, it has been used in place of $\rho_{s}$ in the analysis of this chapter. 
A second factor distinguishing (5.18) and (5.21) comes from density constraints. While allowing an infinitely deep reference level and constant e-folding depth produced the density conservation principle (5.31), the equivalent surface condition in Marshall's work, (5.33), provides no simple constraint. As Marshall notes, however, a density conservation criterion must be imposed at one depth. He chooses to apply it at the bottom, requiring that $\mathbf{u}_{b} \cdot \nabla \rho_{b}=0$. (Since depth, $H$, varies, the condition should be a constraint on potential density rather than density, so the conservation condition Marshall imposes is not exactly correct.) An expression for $\mathbf{u}_{b}$ is obtained by linearizing the velocity about the level of no motion $H_{\text {ref }}$. Thus

$$
\mathbf{u}_{b}=-\frac{g}{f \rho_{o}} \mathbf{k} \wedge \nabla\left[\rho_{b}\left(H-H_{r e f}\right)\right]
$$

which, subject to the bottom density constraint, $\mathbf{u}_{b} \cdot \nabla \rho_{b}=0$, becomes:

$$
\mathbf{u}_{b}=-\frac{g\left(H-H_{r e f}\right)}{f \rho_{o}} \mathbf{k} \wedge \nabla \rho_{b} .
$$

Using this expression for bottom velocity ultimately allows Marshall to derive (5.21). 


\section{Chapter 6}

\section{Summary Discussion}

The preceding chapters have investigated the mean surface structure of the Antarctic Circumpolar Current, its variability, and the dynamical processes controlling its momentum and potential vorticity budgets.

Variability measurements from the Geosat altimeter were used to reconstruct the mean sea surface height across the ACC using a nonlinear iterative fitting procedure. The results are consistent with the presence of two continuous narrow jets encircling the Antarctic continent. These correspond to the Subantarctic and Polar Fronts identified in hydrographic measurements. Their paths are steered around the major topographic features which intersect the flow, in particular Drake Passage, Kerguelen Island, and Campbell Plateau. Smaller subsurface topographic features also affect the flow, notably the Eltanin-Udintsev Fracture Zone in the mid-Pacific, Macquarie Ridge south of New Zealand, and the Crozet Plateau south of Africa. The mean latitude of the ACC varies substantially with longitude and the width of the ACC varies correspondingly, in accord with the Rossby radius's dependence on the Coriolis parameter. Since mean transport is conjectured to be the same along the circumpolar path, this may indicate that the vertical structure deepens as the jet narrows at higher latitudes. 
Although the reconstructed mean sea surface height suggests that the ACC fronts are circumpolar in extent, the variability investigated in chapter 3 indicates relative short mesoscale length scales in the Southern Ocean. Empirical orthogonal functions (EOFs) imply little large scale coherence in surface transport variability, suggesting that the response to wind forcing is local rather than global in extent. In addition harmonic analyses show no evidence for large scale coherent propagation of surface transport anomalies. Thus fluctuations in wind forcing appear to be dissipated locally over $1000 \mathrm{~km}$ length scales rather than traveling along the length of the circumpolar current.

To probe the dynamics governing the ACC in more specific detail, output from the $\frac{1}{4}^{\circ}$ Semtner-Chervin numerical model was examined. The dominant momentum balance in the model is between surface wind stress and bottom form drag. Averaging circumpolarly along a streamline shows that horizontal transfer of momentum is achieved primarily via biharmonic friction in the model ACC, while horizontal eddy flux divergences play a less important role. The relative importance of each term in the momentum balance does not vary substantially along streamlines in the model output; however, the variability of each of the terms in the momentum balance, and correspondingly, the eddy kinetic energy, is substantially greater than its background level at three locations where the model ACC intensifies into a narrow jet, just downstream of Drake Passage, Kerguelen Island, and Campbell Plateau. Surface eddy kinetic energy measured by the altimeter is about twice as large as model values and peaks at many more locations than the model eddy kinetic energy, suggesting that topographic features which do not breach the surface may nonetheless act as sources of eddy kinetic energy. Since kinetic energy increases may be manifestations of topographic lee waves spun up as the ACC meanders, these differences between the model and ocean energies imply that communication between the surface and depth may be more effective in the real ocean than in the model. Overall the results suggest either that too much momentum is diffused above the sill depth and not enough is upwelled 
from depth in the model or that subgrid scale eddy processes are not fully captured by the model.

Finally potential vorticity has been investigated. For unforced flow, potential vorticity is conserved along streamlines. Similarly, if forcing is locally balanced, potential vorticity will also be conserved along streamlines. Conversely the degree to which its value fluctuates indicates how inhomogeneous forcing and dissipation are. In the Semtner-Chervin model, potential vorticity decreases gradually across ocean basins in response to wind stress curl. All potential vorticity input by the wind is dissipated at major topographic features. While surface measurements do not readily lend themselves to potential vorticity analysis, altimeter data suggest that wind stress curl may be balanced by topographic torques at a number of mid-sized topographic features.

The analyses in this study have underscored the importance of topography in steering the ACC, in generating eddy kinetic energy, in dissipating wind forcing, and possibly in breaking up large scale coherent structure. While the numerical model indicates that all topographic effects result from just three features, all of which breach the surface, altimeter data suggests that many smaller topographic ridges also steer the flow and serve as sources of form drag. The importance of mid-sized topography is not explicitly predicted by Treguier and McWilliams [1990] who found that the largest topographic features had the most dramatic impact on the transport of the ACC. Moreover, Wang's [1993] speculation that Drake Passage controls the entire ACC transport does not appear to be justified by the altimeter measurements' indication that numerous topographic features are important. This discrepancy does not necessarily discredit Wang's theory: he suggested that ridges exceeding roughly a critical height of roughly $800 \mathrm{~m}$ and confined within a finite width channel should be sources of form drag. As all of the mid-ocean ridges rise at least a kilometer above the abyssal plain, they may all exceed the critical height and act as significant sources of form drag in the ocean. 
While ACC process studies have not necessarily anticipated the apparent importance of medium-sized topographic features, several recent investigations have examined the critical role bathymetry may play in defining general ocean circulation: Holloway [1992] suggested that the basic barotropic flow should follow topographic contours. Bogden et al. [1993] found that including full topography in an inverse model of the North Atlantic and constraining it to allow no flow through the bottom resulted in circulation which differed substantially from previous inverse estimates. Analytic work by Marshall [1994a] and Salmon [1994] has shown that solutions to the equations of motion may be derived using the method of characteristics, and that bottom topography substantially influences the resulting flow. All of these studies suggest methods for further examining the function of bathymetry in the ACC.

Since mid-sized bathymetric features appear to have a measureable impact on the ACC, we can conjecture that the isopycnal tilting supported by these features and the corresponding meridional flow are important parts of the ACC momentum balance. Ultimately this suggests that thorough understanding of ACC surface circulation will require accurate knowledge of the deep density structure. Conversely, if the surface and deep flow are inextricably linked, then we may hope to monitor the entire circumpolar system merely by measuring the surface conditions.

No single study can hope to explain everything. The results of this thesis suggest that the ACC exists as at least two meandering circumpolar jets, steered by bathymetry. Surface wind stress appears to be dissipated through topographic form drag provided primarily by the major ridge structures which intersect the flow, with additional contributions from medium-sized mid-ocean ridges. The meridional transfer of momentum across the ACC may be due to transient eddies which are subgrid scale in the current version of the Semtner-Chervin model, but the results of this investigation do not indicate whether these transfers should occur near the surface or below the topographic sill depth. The ongoing TOPEX-POSEIDON and ERS-1 altimeter missions coupled with new World Ocean Circulation Experiment (WOCE) 
hydrographic data across the ACC and improving numerical model results should provide the information necessary to improve this picture of ACC circulation. 


\section{Bibliography}

Baker, D. J., Jr., 1982. A note on Sverdrup balance in the Southern Ocean. J. Mar. Res., 40S, 21-26.

Barth, N. and C. Wunsch, 1990. Oceanographic experiment design by simulated annealing. J. Phys. Oceanogr., 20, 1249-1263.

Bogden, P. S., R. E. Davis, and R. Salmon, 1993. The North Atlantic circulation: Combining simplified dynamics with hydrographic data. J. Mar. Res., 51, 1-52.

Bottero, J. S., H. L. Bryden, D. C. Root, and J. Simpkins III, 1981. A Compilation of Observations from Moored Current Meters, XIII. Technical Report "88, Ref. 81-8", Ore. State U., Corvallis, Ore.

Bretherton, F. P., R. E. Davis, and C. B. Fandry, 1976. A technique for objective analysis and design of oceanographic experiments applied to MODE-73. Deep Sea Res., 23, 559-582.

Broecker, W. S., 1987. The biggest chill. Nat. Hist. Mag., 97, 74-82.

Broecker, W. S., 1991. The great ocean conveyor. Oceanography, 4, 79-89.

Bryden, H. L., 1983. The Southern Ocean. In Robinson, A. R., editor, Eddies in Marine Science, pages 265-277. Springer-Verlag, Berlin.

Bryden, H. L. and R. A. Heath, 1985. Energetic eddies at the northern edge of the Antarctic Circumpolar Current in the southwest Pacific. Prog. Oceanog., 14, 65-87.

Callahan, J. E., 1971. Velocity structure and flux of the Antarctic Circumpolar Current south of Australia. J. Geophys. Res., 76, 5859-5864.

Campbell, J. W., 1988. Evaluation of GEOSAT Data and Application to Variability of the Northeast Pacific Ocean. Master's thesis, MIT/WHOI, Woods Hole Oceanogr. Inst., Woods Hole.

Caruso, M. J., Z. Sirkes, P. J. Flament, and M. K. Baker, 1990. Altimeter processing tools for analyzing mesoscale ocean features. Technical Report WHOI-90-45, Woods Hole Oceanogr. Inst., Woods Hole, Mass.

Chelton, D. B., 1982. Statistical reliability and the seasonal cycle: comments on "Bottom pressure measurements across the Antarctic Circumpolar Current and their relation to the wind". Deep Sea Res., 29, 1381-1388. 
Chelton, D. B., 1988. WOCE/NASA Altimeter Algorithm Workshop. U.S. WOCE Technical Report 2, U.S. Planning Office for WOCE, College Station, TX.

Chelton, D. B., M. G. Schlax, D. L. Witter, and J. G. Richman, 1990. Geosat altimeter observations of the surface circulation of the Southern Ocean. $J$. Geophys. Res., 95, 17877-17903.

Cheney, R. E., N. S. Doyle, B. C. Douglas, R. W. Agreen, L. Miller, E. L. Timmerman, and D. C. McAdoo, 1991. The complete Geosat altimeter GDR handbook. Noaa manual nos-ngs-7, U.S. Govt. Print. Office, Washington, D.C. 79 pp.

Danabasoglu, G., J. C. McWilliams, and P. R. Gent, 1994. The role of mesoscale tracer transports in the global ocean circulation. Science, 264, 1123-1126.

Davis, R. E., 1976. Predictability of sea surface temperature and sea level pressure anomalies over the North Pacific Ocean. J. Phys. Oceangr., 6, 249-266.

deSzoeke, R. A. and M. D. Levine, 1981. The advective flux of heat by mean geostrophic motions in the southern ocean. Deep Sea Res., 28, 1057-1085.

Ferraz-Mello, S., 1981. Estimation of periods from unequally spaced observations. Astron. J., 86, 619-624.

Fofonoff, N., 1954. A steady flow in a frictionless homogeneous ocean. J. Mar. Res., $13,254-262$.

FRAM group, 1991. An eddy-resolving model of the Southern Ocean. EOS, 72, $169-175$.

Georgi, D. T. and J. M. Toole, 1982. The Antarctic Circumpolar Current and the oceanic heat and freshwater budgets. J. Mar. Res., 40S, 183-197.

Gill, A. E., 1968. A linear model of the Antarctic Circumpolar Current. J. Fluid Mech., 32, 465-488.

Gill, A. E., 1982. Atmosphere-Ocean Dynamics. Academic Press, New York.

Gill, A. E. and K. Bryan, 1971. Effects of geometry on the circulation of a threedimensional southern-hemisphere ocean model. Deep Sea Res., 18, 685-721.

Gill, A. E. and P. P. Niiler, 1973. The theory of the seasonal variability in the ocean. Deep Sea Res., 20, 141-177.

Gille, S. T., 1994. Mean sea surface height of the Antarctic Circumpolar Current from Geosat data: Method and application. J. Geophys. Res., 99, 18,25518,273 .

Gordon, A. L., 1967. Structure of Antarctic waters between $20^{\circ} \mathrm{W}$ and $170^{\circ} \mathrm{W}$. In Bushnell, V., editor, Antarctic Map Folio Ser. Am. Geogr. Soc., New York. folio 6 .

Gordon, A. L., 1972. On the interaction of the Antarctic Circumpolar Current and the Macquarie Ridge. In Hayes, D. E., editor, Antarctic Oceanology II: The Australian-New Zealand Sector, Antarctic Res. Ser., volume 19, pages 71-78. AGU, Washington, D.C. 
Gordon, A. L., E. Molinelli, and T. Baker, 1978. Large-scale relative dynamic topography of the Southern Ocean. J. Geophys. Res., 83, 3023-3032.

Gordon, A. L., E. J. Molinelli, and T. N. Baker, 1982. Southern Ocean Atlas. Columbia University Press, New York.

Holloway, G., 1992. Representing topographic stress for large-scale ocean models. J. Phys. Oceangr., 22, 1033-1046.

Inoue, M., 1985. Modal decomposition of the low-frequency currents and baroclinic instability at Drake Passage. J. Phys. Oceangr., 15, 1157-1181.

Ivchenko, V. O., K. J. Richards, and D. P. Stevens, 1994. The dynamics of the Antarctic Circumpolar Current. J. Phys. Oceanogr. submitted.

Jacobs, S. S. and D. T. Georgi, 1977. Observations on the southwest Indian/Antarctic Ocean. In $A$ Voyage of Discovery. Pergamon, New York.

Jacobs, G. A., G. H. Born, M. E. Parke, and P.C. Allen, 1992. The global structure of the annual and semiannual sea surface height variability from Geosat altimeter data. J. Geophys. Res., 97, 17813-17828.

Johnson, G. C. and H. L. Bryden, 1989. On the size of the Antarctic Circumpolar Current. Deep Sea Res., 36, 39-53.

Johnson, T. J., R. H. Stewart, C. K. Shum, and B. D. Tapley, 1992. Distribution of Reynolds stress carried by mesoscale variability in the Antarctic Circumpolar Current. Geophys. Res. Lett., 19, 1201-1204.

Joyce, T. M. and S. L. Patterson, 1977. Cyclonic ring formation at the Polar Front in the Drake Passage. Nature, 265, 131-133.

Kelly, K. A., 1988. Comment on "empirical orthogonal function analysis of Advanced Very High Resolution Radiometer surface temperature patterns in Santa Barbara Channel" by G. S. E. Lagerloef and R. L. Bernstein. J. Geophys. Res., 93, 15,753-15,754.

Kelly, K. A., 1991. The meandering Gulf Stream as seen by the Geosat altimeter: Surface transport, position, and velocity variance from $73^{\circ}$ to $46^{\circ} \mathrm{W}$. J. Geophys. Res., 96, 16,721-16,738.

Kelly, K. A. and S. T. Gille, 1990. Gulf Stream surface transport and statistics at $69^{\circ} \mathrm{W}$ from the Geosat altimeter. J. Geophys. Res., 95, 3149-3161.

Kelly, K. A. and D. R. Watts, 1994. Monitoring Gulf Stream transport by radar altimeter and inverted echo sounders. J. Phys. Oceanogr., 24, 1080-1084.

Killworth, P. D., 1992. An equivalent-barotropic mode in the Fine Resolution Antarctic Model. J. Phys. Oceanogr., 22, 1379-1387.

Killworth, P. D. and M. M. Nanneh, 1994. Isopycnal momentum budget of the Antarctic Circumpolar Current in the Fine Resolution Antarctic Model. $J$. Phys. Oceanogr., 24, 1201-1223. 
Killworth, P. D., D. Stainforth, D. J. Webb, and S. M. Paterson, 1991. The development of a free-surface Bryan-Cox-Semtner ocean model. J. Phys. Oceanogr., 21, 1331-1348.

Klinck, J. M., 1992. Effects of wind, density, and bathymetry on a one-layer Southern Ocean model. J. Geophys. Res., 97, 20,179-20,189.

Large, W. G. and H. van Loon, 1989. Large scale, low frequency variability of the 1979 FGGE surface buoy drifts and winds over the Southern Hemisphere. $J$. Phys. Oceanogr., 19, 216-232.

Lutjeharms, J. R. E., 1977. Meso-scale Dynamics in the Southern Ocean: a Statistical Analysis of Historic Data. $\mathrm{PhD}$ thesis, U. of Washington.

Lutjeharms, J. R. E. and D. J. Baker, Jr., 1980. A statistical analysis of the mesoscale dynamics of the Southern Ocean. Deep Sea Res., 27A, 145-159.

Macdonald, A. M., 1993. Property fluxes at $30^{\circ} \mathrm{S}$ and their implications for the Pacific-Indian throughflow and the global heat budget. J. Geophys. Res., 98, 6851-6868.

Marshall, D., 1994a. Influence of topography on the large-scale ocean circulation. J. Phys. Oceanogr. submitted.

Marshall, D., 1994b. Topographic steering of the Antarctic Circumpolar Current. J. Phys. Oceanogr. submitted.

Marshall, J., D. Olbers, H. Ross, and D. Wolf-Gladrow, 1993. Potential vorticity constraints on the dynamics and hydrography of the Southern Ocean. J. Phys. Oceanogr., 23, 465-487.

McDougall, T. J., 1989. Streamfunctions for the lateral velocity vector in a compressible ocean. J. Mar. Res., 47, 267-284.

McWilliams, J. C., W. R. Holland, and J. H. S. Chow, 1978. A description of numerical Antarctic Circumpolar Currents. Dyn. Atmos. Oceans, 2, 213-291.

Mertz, G. and D. G. Wright, 1992. Interpretations of the JEBAR term. J. Phys. Oceanogr., 22, 301-305.

Mestas-Nuñez, A. M., D. B. Chelton, M. H. Freilich, and J. G. Richman, 1994. An evaluation of ECMWF-based climatological wind stress fields. J. Phys. Oceanogr., 24, 1532-1549.

Minster, J. F. and M. C. Gennero, 1995. High frequency space-time variability of Western boundary currents using ERS-1 altimeter data. J. Geophys. Res., page in press.

Morrow, R., J. Church, R. Coleman, D. Chelton, and N. White, 1992. Eddy momentum flux and its contribution to the Southern Ocean momentum balance. Nature, 357, 482-484.

Morrow, R., R. Coleman, J. Church, and D. Chelton, 1994. Surface eddy momentum flux and velocity variances in the Southern Ocean from Geosat altimetry. $J$. Phys. Oceanogr., 24, 2050-2071. 
Munk, W. H., 1950. On the wind-drive ocean circulation. J. Met., 7, 79-93.

Munk, W. H. and E. Palmén, 1951. Note on the dynamics of the Antarctic Circumpolar Current. Tellus, 3, 53-55.

Nowlin, W. D., Jr. and M. Clifford, 1982. The kinematic and thermohaline zonation of the Antarctic Circumpolar Current at Drake Passage. J. Mar. Res., 40S, 481-507.

Nowlin, W. D., Jr. and J. M. Klinck, 1986. The physics of the Antarctic Circumpolar Current. Rev. Geophys., 24, 469-491.

Olbers, D., V. Gouretski, G. Seiß, and J. Schröter, 1992. Hydrographic Atlas of the Southern Ocean. Alfred Wegener Institute, Bremerhaven, Germany.

Olbers, D., C. Wübber, and J.-O. Wolff, 1992. The dynamical balance of wind and buoyancy driven circumpolar currents. Technical Report "Technical Report 32", Alfred-Wegener-Institut für Polar-und Meeresforschung, Bremerhaven, Germany.

Orsi, A. H., 1993. On the extent and frontal structure of the Antarctic Circumpolar Current. PhD thesis, Texas A\&M University, College Station, Tex.

Pacanowski, R. C. and S. G. H. Philander, 1981. Parameterization of vertical mixing in numerical models of tropical oceans. J. Phys. Oceanogr., 11, 1443-1451.

Park, Y.-H., L. Gambéroni, and E. Charriaud, 1991. Frontal structure and transport of the Antarctic Circumpolar Current in the south Indian Ocean sector, $40^{\circ}-$ $80^{\circ}$ E. Mar. Chem., 35, 45-62.

Park, Y.-H., L. Gambéroni, and E. Charriaud, 1993. Frontal structure, water masses, and circulation in the Crozet Basin. J. Geophys. Res., 98, 12,361-12,385.

Patterson, S. L., 1985. Surface circulation and kinetic energy distributions in the Southern Hemisphere oceans from FGGE drifting buoys. J. Phys. Oceanogr., $15,865-884$.

Pedlosky, J., 1987. Geophysical Fluid Dynamics. Springer-Verlag, New York, second edition.

Peterson, R. G. and T. Whitworth, 1989. The subantarctic and polar fronts in relation to deep water masses through the southwestern Atlantic. J. Geophys. Res., 94, 10,817-10,838.

Peterson, R. G., W. D. Nowlin, and T. Whitworth, 1982. Generation and evolution of a cyclonic ring at Drake Passage in early 1979. J. Phys. Oceanogr., 12, 712-719.

Pillsbury, R. D., T. Whitworth III, W. D. Nowlin, Jr., and F. Sciremammano, Jr., 1979. Currents and temperatures as observed in Drake Passage during 1975. $J$. Phys. Oceanogr., 9, 469-482.

Pillsbury, R. D., Jr. F. Sciremammano, J. S. Bottero, and R. E. Still, 1980. A Compilation of Observations from Moored Current Meters, XII. Technical Report 82, Ref. 80-11, Ore. State U., Corvallis, Ore. 
Pillsbury, R. D., J. S. Bottero, and R. E. Still, 1981. A Compilation of Observations from Moored Current Meters, XIV A. Technical Report "91, Ref. 81-17", Ore. State U., Corvallis, Ore.

Preisendorfer, R. W., 1988. Principal Component Analysis in Meteorology and Oceanography. Elsevier, New York. 425 pp.

Press, W. H., B. P. Flannery, S. A. Teukolsky, and W. T. Vetterling, 1986. Numerical Recipes. Cambridge University Press, New York. 818 pp.

Qiu, B., 1992. Recirculation and seasonal change of the Kuroshio from altimetry observations. J. Geophys. Res., 97, 17,801-17,811.

Qiu, B., Kelly K. A., and T. M. Joyce, 1991. Mean flow and variability of the Kuroshio Extension from Geosat altimetry data. J. Geophys. Res., 96, 18,49118,507 .

Read, J. F. and R. T. Pollard, 1993. Structure and transport of the Antarctic Circumpolar Current and Agulhas Return Current at $40^{\circ}$ E. J. Geophys. Res., 98, 12,281-12,295.

Reid, J. L., Jr., 1961. On the temperature, salinity, and density differences between the Atlantic and Pacific oceans in the upper kilometre. Deep Sea Res., 7, 265275.

Reid, J. L., 1986. On the total geostrophic circulation of the South Pacific Ocean: Flow patterns, tracers and transports. Prog. in Oceanogr., 16, 1-61.

Reid, J. L., 1989. On the total geostrophic circulation of the South Atlantic Ocean: Flow patterns, tracers and transports. Prog. in Oceanogr., 23, 149-244.

Reid, J. L. and R. J. Lynn, 1971. On the influence of the Norwegian-Greenland and Weddell seas upon the bottom waters of the Indian and Pacific oceans. Deep Sea Res., 18, 1063-1088.

Rintoul, S. R., 1991. South Atlantic interbasin exchange. J. Geophys. Res., 96, 2675-2692.

Salmon, R., 1994. Generalized two-layer models of ocean circulation. page in preparation.

Saunders, P. M. and S. R. Thompson, 1993. Transport, heat, and freshwater fluxes within a diagnostic numerical model (FRAM). J. Phys. Oceanogr., 23, 452-464.

Savchenko, V. G., W. J. Emery, and O. A. Vladimirov, 1978. A cyclonic eddy in the Antarctic Circumpolar Current south of Australia: Results of Soviet-American observations aboard the R/V Professor Zubov. J. Phys. Oceanogr., 8, 825-837.

Schmitz, W. J., Jr., 1994. On the global scale thermohaline circulation. Rev. Geophys. submitted.

Sciremammano, F., Jr., R. D. Pillsbury, Jr. W. D. Nowlin, and T. Whitworth III, 1980. Spatial scales of temperature and flow in Drake Passage. J. Geophys. Res., 85, 4015-4028. 
Semtner, A. J., 1986. Finite-difference formulation of a world ocean model. In O'Brien, J. J., editor, Proceedings of the NATO Advanced Physical Oceanographic Numerical Modelling. Reidel, Dordrecht.

Semtner, A. J. and R. M. Chervin, 1992. Ocean general circulation from a global eddy-resolving model. J. Geophys. Res., 97, 5493-5550.

Shum, C. K., R. A. Wender, D. T. Sandwell, B. H. Zhang, R. S. Nerem, and B. D. Tapley, 1990. Variations of global mesoscale eddy energy observed from Geosat. J. Geophys. Res., 95, 17,865-17,876.

Stammer, D. and C. W. Böning, 1992. Mesoscale variability in the Atlantic Ocean from Geosat altimetry and WOCE high-resolution numerical modeling. J. Phys. Oceanogr., 22, 732-752.

Stammer, D. and C. Wunsch, 1994. Preliminary assessment of the accuracy and precision of TOPEX/POSEIDON altimeter data with respect to the large-scale ocean circulation. J. Geophys. Res., 99, 24,584-24,604.

Stevens, D. P. and V. O. Ivchenko, 1994. The zonal momentum balance in a realistic eddy resolving general circulation model of the Southern Ocean. J. Phys. Oceanogr. submitted.

Stommel, H., 1948. The westward intensification of wind-driven ocean currents. Trans. Amer. Geoph. Union, 29, 202-206.

Stommel, H., 1957. A survey of ocean current theory. Deep Sea Res., 4, 149-184.

Taylor, J. R., 1982. An Introduction to Error Analysis: The Study of Uncertainties in Physical Measurements. University Science Books, Mill Valley, CA. 270 pp.

Thompson, S. R., 1993. Estimation of the transport of heat in the Southern Ocean using a Fine-Resolution Numerical Model. J. Phys. Oceanogr., 23, 2493-2497.

Toggweiler, J. R. and B. Samuels, 1994. Effect of Drake Passage on the global thermohaline circulation. Deep Sea Res., page submitted.

Treguier, A. M. and J. C. McWilliams, 1990. Topographic influences on winddriven stratified flow in a $\beta$-plane channel: an idealized model for the Antarctic Circumpolar Current. J. Phys. Oceanogr., 20, 321-343.

Trenberth, K. E., J. G. Olson, and W. G. Large, 1989. A global ocean wind stress climatology based on ECMWF analyses. Technical Report NCAR/TN-338+STR, Natl. Cent. for Atmos. Res., Boulder, Colo. 93 pp.

van Loon, H., 1972. Half-yearly oscillations in the Drake Passage. Deep Sea Res., $19,525-527$.

Vassie, J. M., A. J. Harrison, P. L. Woodworth, S. A. Harangazo, and M. J. Smithson, 1994. On the temporal variability of the transport between Amsterdam and Kerguelen Islands. J. Geophys. Res., 99, 937-949.

Wang, L., 1993. The Dynamic Role of Ridges in a $\beta$-Plane Channel: Towards understanding the dynamics of large scale circulation in the Southern Ocean. $\mathrm{PhD}$ thesis, MIT/WHOI Joint Program, Woods Hole, Mass. 
Wearn, R. B., Jr. and D. J. Baker Jr., 1980. Bottom pressure measurements across the Antarctic Circumpolar Current and their relation to the wind. Deep Sea Res., 27A, 875-888.

Wells, N. C. and B. A. de Cuevas, 1994. Vorticity dynamics of the Southern Ocean from a General Circulation Model. J. Phys. Oceanogr. submitted.

Whitworth, I., T., 1983. Monitoring the transport of the Antarctic Circumpolar Current at Drake Passage. J. Phys. Oceanogr., 13, 2045-2057.

Whitworth, I., T. and R. G. Peterson, 1985. Volume transport of the Antarctic Circumpolar Current from Bottom Pressure Measurements. J. Phys. Oceanogr., $15,810-816$.

Whitworth, I., T., W. D. Nowlin, Jr., and S. J. Worley, 1982. The net transport of the Antarctic Circumpolar Current through Drake Passage. J. Phys. Oceanogr., 12, 960-971.

Whitworth, I., T., R. D. Pillsbury, M. I. Moore, and R. F. Weiss, 1991. Observations of the Antarctic Circumpolar Current and deep boundary current in the Southwest Atlantic. J. Geophys. Res., 96, 15,105-15,118.

Wilkin, J. L. and R. A. Morrow, 1994. Eddy kinetic energy and momentum flux in the Southern Ocean: Comparison of a global eddy-resolving model with altimeter, drifter, and current-meter data. J. Geophys. Res., 99, 7903-7916.

Wolff, J.-O., E. Maier-Reimer, and D. J. Olbers, 1991. Wind-driven flow over topography in a zonal $\beta$-plane channel: A quasi-geostrophic model of the Antarctic Circumpolar Current. J. Phys. Oceanogr., 21, 236-264.

Wright, D. G. and K. R. Thompson, 1983. Time-averaged forms of the nonlinear stress law. J. Phys. Oceanogr., 13, 341-345.

Wunsch, C., 1989. Sampling characteristics of satellite orbits. J. Atmos. Ocean. Tech., 6, 891-907.

Zhang, H.-M. and N. G. Hogg, 1992. Circulation and water mass balance in the Brazil Basin. J. Mar. Res., 50, 385-420. 


\section{Short Abstract}

Geosat altimeter data and numerical model output are used to examine the circulation and dynamics of the Antarctic Circumpolar Current (ACC). The mean sea surface height across the ACC has been reconstructed from height variability measured by the altimeter, without assuming prior knowledge of the geoid. The results indicate locations for the Subantarctic and Polar Fronts which are consistent with in situ observations and indicate that the fronts are substantially steered by bathymetry. Detailed examination of spatial and temporal variability indicates a spatial decorrelation scale of $85 \mathrm{~km}$ and a temporal e-folding scale of 34 days. Empirical Orthogonal Function analysis suggests that the scales of motion are relatively short, occuring on $1000 \mathrm{~km}$ length-scales rather than basin or global scales. The momentum balance of the ACC has been investigated using output from the high resolution primitive equation model in combination with altimeter data. In the Semtner-Chervin quarter-degree general circulation model topographic form stress is the dominant process balancing the surface wind forcing. In stream coordinates, the dominant effect transporting momentum across the ACC is biharmonic friction. Potential vorticity is considered on Montgomery streamlines in the model output and along surface streamlines in model and altimeter data. 